\title{
RF-AMIDE NEUROPEPTIDES AND THEIR RECEPTORS IN MAMMALS : PHARMACOLOGICAL PROPERTIES, DRUG DEVELOPMENT AND MAIN PHYSIOLOGICAL FUNCTIONS
}

\author{
Raphaelle QUILLET ${ }^{\text {a, }}$, Safia AYACHI ${ }^{\text {a, }}{ }^{\text {, Fédéric BIHEL }}{ }^{\text {b }}$, Khadija ELHABAZI ${ }^{\text {a }}$, \\ Brigitte ILIEN $^{\text {a, }}$ and Frédéric SIMONIN ${ }^{\text {a, }}$, *
}

\section{Affiliations :}

a) Biotechnologie et Signalisation Cellulaire, UMR 7242 CNRS, Université de Strasbourg, Illkirch, France

b) Laboratoire Innovation Thérapeutique, UMR 7200 CNRS, Université de Strasbourg, Illkirch, France

,$+ \S$ : Equally contributing authors

* Corresponding author :

Frédéric Simonin

Biotechnologie et Signalisation Cellulaire, UMR 7242 CNRS/UdS

Ecole Supérieure de Biotechnologie de Strasbourg, 300, Boulevard Sébastien Brant, BP 10413 67412 Illkirch Cedex, France.

Tel : +33 3688548 75; Fax : +33 368854683 . 


\begin{abstract}
RF-amide neuropeptides, with their typical Arg-Phe- $\mathrm{NH}_{2}$ signature at their carboxyl C-termini, belong to a lineage of peptides that spans the almost entire life tree. Throughout evolution, RFamide peptides and their receptors preserved fundamental roles in reproduction and feeding, both in Vertebrates and Invertebrates. The scope of this review is to summarize the current knowledge on the RF-amide systems in Mammals from historical aspects to therapeutic opportunities. Taking advantage of most recent findings in the field, special focus will be given on molecular and pharmacological properties of RF-amide peptides and their receptors as well as on their implication in the control of different physiological functions including feeding, reproduction and pain. Recent progress on the development of drugs that target RF-amide receptors will also be addressed.
\end{abstract}

\title{
Keywords
}

RF-amide neuropeptides $\ddot{i}$ RF-amide receptors $і ̈$ feeding $\ddot{i}$ reproduction $і ̈$ pain $і ̈$ drug discovery. 


\section{Table of Contents :}

1. Introduction

2. RF-amide neuropeptides and their precursors in Mammals

3. Mammalian RF-amide receptors

4. RF-amides in vitro pharmacology : Ligand-receptor binding properties

5. RF-amides in vitro pharmacology : Intracellular signalling pathways

6. Structure-activity relationships studies on mammalian RF-amide peptides and non-peptidic ligands for RF-amide receptors

7. Mammalian RF-amides peptides and their receptors in feeding and metabolism

8. Mammalian RF-amide peptides and their receptors in reproduction

9. Mammalian RF-amide peptides and their receptors in nociception and pain

10. Conclusion and therapeutic perspectives

Conflict of interest

Acknowledgments

References 


\section{Abbreviations}

AHA, anterior hypothalamic area; Amd, amygdala; AMPA, Ŭ-amino-3-hydroxy-5-methyl-4isoxazolepropionic acid; AP, area postrema; ARC, arcuate nucleus; AVPV, anteroventral periventricular hypothalamic nucleus; BST, bed nucleus of stria terminalis; CCK, cholecystokinin; $\mathrm{CHO}$, chinese hamster ovary cells; COS, fibroblast-like cell line derived from monkey kidney tissue; $\mathrm{CPu}$, caudate putamen; $\mathrm{CREB}$, cAMP response element-binding protein; $\mathrm{DMH}$, dorsomedial hypothlamic nucleus; DMR, dorsal or median raphe nuclei; DMX, dorsal motor nucleus of vagus; DRG, dorsal root ganglia; ERK, extracellular signal-regulated kinase; FSH, follicle stimulating hormone; GnIH, GnRH inhibitory hormone; GnRH, gonadotropin-releasing hormone; GPCR, G protein-coupled receptor; Hab, habenula; HEK, human enmbryonic kidney cells; HPG, hypothalamic-pituitary-gonadal axis; IHC, immunohistochemistry; ir, immunoreactive; ISH, in situ hybridization; JNK, c-Jun N-terminal kinase; Kiss, kisspeptin; Kiss1R, kisspeptin receptor; KO, knockout; LC, locus coeruleus; LH, luteinizing hormone; LHA, lateral hypothalamic area; LSN, lateral septal nucleus; MAPK, mitogen-activated protein kinase; mTOR, mammalian target of rapamycin; $\alpha-\mathrm{MSH}$, melanocyte-stimulating hormone; NAc, nucleus accumbens; NPAF, neuropeptide AF; NPFF, neuropeptide FF; NPSF, neuropeptide SF; NPFF1R, neuropeptide FF receptor 1; NPFF2R, neuropeptide FF receptor 2; NPY, neuropeptide Y; NTS, nucleus tractus solitarius; OB, olfactory bulb; OFC, orbitofrontal cortex; OVX, ovariectomized; PAG, periaqueductal gray; PB, parabrachial nucleus; PC12, rat pheochromocytoma cells; PeV, periventricular hypothalamic nucleus; PKA, protein kinase A; PKC, proteine kinase C; PLC, phospholipase C; POA, preoptic area; POMC, proopio-melanocortin; PRL, prolactin hormone; PrRP, prolactin-releasing peptide; PT, pars tuberalis; PTX, pertussis toxin; PVN, paraventricular hypothalamic nucleus; QRFP-R, QRFP receptor; QRFP-R1, rodent QRFP receptor isoform 1; QRFP-R2, rodent QRFP receptor isoform 2; RFRP, RF-amide related peptide; RFRP-R, RFRP receptor; SAR, structure-activity/affinity relationship; 7TM, seven transmembrane; SCN, suprachiasmatic nucleus; SON, supraoptic nucleus; STN, spinal trigeminal nucleus; SuM, supramammillary nucleus; TM, tuberomammillary nucleus; VLM, ventrolateral medulla; VMH, ventromedial hypothalamic nucleus; VPL, ventral postlateral thalamic nucleus. VTA, ventral tegmental area. 


\section{Introduction}

The isolation in 1977 of the cardio-excitatory peptide Phe-Met-Arg-Phe- $\mathrm{NH}_{2}\left(\mathrm{FMRF}-\mathrm{NH}_{2}\right)$ from the ganglia of the Macrocalista nimbosa clam (Price \& Greenberg, 1977) and the subsequent identification of FMRF- $\mathrm{NH}_{2}$-like immunoreactivity in insect, fish, amphibian, avian and mammalian tissues (Boer et al., 1980; Dockray et al., 1981; Weber et al., 1981; Dockray et al., 1983) paved the way to the discovery of a wide family of neuropeptides present in all major animal phyla (Walker et al., 2009; Jékely 2013). This family is currently referred to as FMRFamide-like peptides or RF-amide peptides, a definition broad enough to encompass a vast repertoire of peptides that display a great sequence diversity but share a typical C-terminal Arg-Phe- $\mathrm{NH}_{2}$ (RF-amide) sequence (Espinoza et al., 2000; Walker et al., 2009).

Although available information varies notably between phyla, bioinformatic analyses of genome and transcriptome databases, cDNA libraries screening and peptidomic strategies enabled the prediction and the identification of numerous RF-amide peptides and receptors in Invertebrates and Vertebrates. The most recent studies indicate that the RF-amide motif is both an ancient and convergent feature of neuropeptides that underwent various evolutionary scenarios (retention, modification or loss of the RF-amide signature) accross the animal kingdom, in parallel or independently from their receptors (Elphick \& Mirabeau, 2014).

In Invertebrates, RF-amides represent a large peptide family. For example, in the nematode C. elegans, up to thirty one genes encoding more than seventy RF-amide peptides have been identified (Walker et al., 2009; Peymen et al., 2014). Functional studies also evidenced a broad spectrum of biological activities for RF-amides in Invertebrates, including regulation of the cardiovascular, respiratory, reproductive and sensory systems as well as learning and feedingrelated behaviours, key functions for these peptides that emerged consistently throughout evolution (Walker et al., 2009; Nässel \& Wegener 2011; Krajniak et al., 2013; Peymen et al., 2014; Elphick \& Mirabeau 2014).

In Vertebrates, only five RF-amide peptide subfamilies encoded by five precursor genes have been identified: (1) neuropeptide FF (NPFF), (2) RF-amide-related peptide (RFRP), (3) prolactin-releasing peptide (PrRP), (4) kisspeptin and (5) pyroglutamylated RF-amide peptide (QRFP). These neuropeptides signal through five distinct G protein-coupled receptors (GPCRs) that belong to the rhodopsin family. Phylogenetic analyses of GPCRs in the human genome revealed the clustering of NPFF (GPR74), RFRP (GPR147), PrRP (GPR10) and QRFP (GPR103) receptors within the $\beta$ group for neuropeptide receptors and the location of kisspeptin (GPR54) receptors within the $\gamma$ group for somatostatin/opioid/galanin receptors (Fredriksson et al., 2003; Elphick \& Mirabeau 2014; Yun et al., 2014). Such a distant evolutionary origin of kisspeptin receptors also 
concerns their endogenous ligand as kisspeptins were proposed to arise from an ancestor common to galanin and spexin (Kim et al., 2014). Although current data support both parallel and independent evolutionary histories for kisspeptins and their receptors in Vertebrates (Pasquier et al., 2012), the kisspeptin system clearly separates from the other RF-amide peptide/receptor pairs (Yun et al., 2014).

In Mammals, the different RF-amide systems have been involved in the modulation of numerous functions including reproduction, cardiovascular function, pain and nociception, feeding and energy homeostasis, reward, as well as in peripheral functions and tumor progression. In this review, we address the current knowledge on RF-amide systems in Mammals from historical aspects to therapeutic opportunities, with a particular focus on their prominent roles in feeding and metabolism, reproduction and nociception.

\section{RF-amide neuropeptides and their precursors in Mammals}

Mammalian RF-amide neuropeptides subdivide into five groups (Table 1) : the neuropeptide FF (NPFF) group, the RF-amide-related peptide (RFRP) group, the prolactin-releasing peptide (PrRP) group, the kisspeptin group and finally the QRFP group (Findeisen et al., 2011a; Fukusumi et al., 2006; Parhar et al., 2012).

\subsection{Mammalian RF-amide neuropeptides}

\subsubsection{The NPFF and NPAF subgroup}

An octapeptide (NPFF) and a 18 amino acid-long peptide (NPAF), both harbouring a typical carboxy-terminal RF-amide signature (Table 1), were first detected in bovine brain using antibodies directed against FMRF-NH 2 , then purified and sequenced (Yang et al., 1985). These neuropeptides were subsequently isolated from human serum (Sundblom et al., 1995) and cerebrospinal fluid (Sundblom et al., 1997; Burlet-Schiltz et al., 2002), rat brain (Yang \& Martin, 1995) and from rat and mouse spinal cord (Yang \& Martin, 1995; Bonnard et al., 2001).

The existence of NPFF-containing peptide with three additional $\mathrm{N}$-terminal amino acids was reported in rat (NPA-NPFF; Bonnard et al., 2001) and mouse (SPA-NPFF; Bonnard et al., 2003) spinal cord as well as in SH-SY5Y cells (SQA-NPFF; Bonnard et al., 2003). Conversely, a shorter NPAF form (SLAAPQRF-amide; often referred to as NPSF), has been isolated in rodent brain and spinal cord (Yang \& Martin, 1995; Bonnard et al., 2001) as well as in human cerebrospinal fluid (Burlet-Schiltz et al., 2002) and SH-SY5Y cells (Bonnard et al., 2003). 
All these peptides contain a common PQRF-amide C-terminal end but may vary in length and sequence depending on mammalian species (Table 1).

\subsubsection{The RF-amide related peptides (RFRPS)}

Gene database searches for additional peptides pointed to human ESTs encoding two putative RF-amide-related peptides (Hinuma et al., 2000; Liu et al., 2001), termed RFRP-1 (or NPSF) and RFRP-3 (or NPVF). Endogenous RFRP-1 and RFRP-3 peptides have been subsequently isolated as mature peptides in bovine (Fukusumi et al., 2001; Yoshida et al., 2003), Siberian hamster (Ubuka et al., 2012a) and in human brain hypothalamic extracts (Ubuka et al., 2009b) and cerebrospinal fluid (Burlet-Schiltz et al., 2002). Yet, only RFRP-3 was biochemically characterized in rat (Ukena et al., 2002) and macaque (Ubuka et al., 2009a).

According to species, RFRP peptides exhibit short or longer N-terminal portions but all of them display a well-conserved C-terminal LPXRF-amide (with X=L in RFRP-1 and Q in RFRP-3) motif (Table 1), as does the gonadotropin-inhibitory hormone $(\mathrm{GnIH})$ identified in quail brain (Tsutsui et al., 2000). RFRP-1 displays closer structural homology with avian GnIH but functional studies tend to indicate that RFRP-3 is most likely the GnIH homolog in Mammals (Clarke et al., 2008; Pineda et al., 2010a; Smith \& Clarke, 2010; Tsutsui et al., 2012; Ubuka et al., 2012b). A consensus view now considers mammalian RFRPs and avian GnIHs as RF-amide peptide orthologs (Kriegsfeld et al., 2006; Smith \& Clarke, 2010).

\subsubsection{The prolactin-releasing peptides (PrRPs)}

Using a reverse pharmacological approach, novel RF-amide peptide members referred to as prolactin-releasing peptides (PrRPs) were identified as ligands of the orphan hGR3 receptor (Hinuma et al., 1998). Their purification from bovine hypothalamic extracts revealed the existence of 20- (PrRP-20) and 31- (PrRP-31) amino acid-long isoforms (Table 1), derived from a common PrRP precursor protein. Highly conserved mature PrRP peptides were also predicted to arise from human, mouse and rat precursors (Hinuma et al., 1998; Tachibana \& Sakamoto, 2014).

\subsubsection{The Kisspeptin peptides (Kisspeptins)}

Independent studies aiming at defining the endogenous ligands of GPR54 (Kotani et al., 2001a), OT7T175 (Ohtaki et al., 2001) and AXOR12 (Muir et al., 2001) orphan receptors, led to the discovery of the kisspeptin RF-amide peptide group (Table 1). Three peptides of 54, 14 and 13 amino-acids were isolated from human placenta, characterized as potent GPR54/ OT7T175 agonists and defined as translation products of the KiSS-1 gene (Kotani et al., 2001a; Ohtaki et al., 2001). Accordingly, these endogenous peptides were defined as kisspeptin-54 (Kp-54; or metastin), 
kisspeptin-14 (Kp-14) and kisspeptin-13 (Kp-13). Additional detection of kisspeptin-10 (Kp-10) in human trophoblasts (Bilban et al., 2004) allowed to confirm that all kisspeptins derive from the KiSS-1 gene, a metastasis suppressor gene (Lee et al., 1996).

\subsubsection{The QRFP peptides (QRFPS)}

The discovery of the last RF-amide peptides group started with the isolation of 26RFa, a neuropeptide from frog brain, and the observation of homologous sequences in human genome and rat ESTs (Chartrel et al., 2003). 26RFa rapidly turned out to correspond to the putative human P518 and mouse P550 peptidic ligands for the orphan SP9155 receptor identified from a series of peptides predicted to originate respectively from human and mouse precursor proteins (Jiang et al., 2003). Following a similar bioinformatic approach, Fukusumi and collaborators identified a human gene candidate encoding a precursor for a 43 amino-acid long peptide, starting from $\mathrm{N}$-terminal pyro-Glu and ending at C-terminal Arg-Phe- $\mathrm{NH}_{2}$, and named it QRFP (Fukusumi et al., 2003). Fulllength QRFP was isolated from the supernatant of CHO cells transfected with human QRFP cDNA and found to behave as a potent agonist of the orphan human AQ27 receptor (QRFP-R).

Endogenous QRFP-26 and QRFP-43 peptides (Table 1) were subsequently identified in rat brain (Takayasu et al., 2006), in human hypothalamus and spinal cord extracts and in PC12 cell lysates (Bruzzone et al., 2006).

\subsection{Precursors for $R F$-amide neuropeptides}

The five groups of RF-amide peptides derive from sequential proteolytic/maturation processing of five distinct prepro-proteins encoded by five distinct precursor genes that contain a single coding exon giving rise to one mRNA transcript (no splice variants). RF-amide precursor proteins share a common structure : a hydrophobic signal sequence at their N-terminal end, paired basic amino acids ( $\mathrm{R}$ and/or $\mathrm{K}$ ) as recognition sites for endoproteases and a $\mathrm{C}$-terminal consensus sequence (RXGR/K) for peptide processing, with Gly as an amide donor (Eipper et al., 1992; Southey et al., 2006). Cleavage (pro protein convertases) and maturation (carboxypeptidase $E$ and peptidyl $\alpha$ amidating monooxygenase) enzymes are likely involved in the processing of RF-amide precursor proteins (Bonnard et al., 2001; Fukusumi et al., 2006; Southey et al., 2006).

\subsubsection{The proNPFF A precursor for $N P F F$ and $N P A F$}

The gene encoding the proNPFFA precursor has been cloned in Mammals (Perry et al., 1997; Vilim et al., 1999) and contains three coding exons. Human, bovine, murine and rat $\operatorname{proNPFF}_{\mathrm{A}}$ precursors (113-115 amino acids long), as deduced from their cDNAs, are highly related 
polypeptides (40\% overall sequence identity). They encompass two putative peptides (NPFF and NPAF) flanked at their C-termini by RFGR (NPFF) or RFGK (NPAF) motifs and by single or paired Arg residues as putative N-terminal cleavage sites (Perry et al., 1997; Vilim et al., 1999; Bonnard et al., 2001, 2003).

According to general processing rules, only N-terminal extended NPFF undecapeptides (i.e. NPA-, SQA- or SPA-NPFF, depending on species) and N-terminal extended NPAF-derived peptides (11 amino-acid long peptides, such as EFW-NPSF in rat or QFW-NPSF in mice (Table 1), which have not been isolated yet, and 18 amino-acid long peptides which were only found in human and bovine tissues) are predicted from proNPFFA processing (Vilim et al., 1999; Bonnard et al., 2001, 2003). Thus, the precursors apparently lacked consensus N-terminal cleavage sites for NPFF and the NPAF-derived octapeptide (SLAAPQRF-amide) and displayed species-dependent Nterminal processing mechanisms. Several hypotheses have been proposed to explain these inconstancies : i) in rodents, and similarly to human and bovine NPAF generation, $\operatorname{proNPFF}_{\mathrm{A}}$ precursor cleavage at a single Arg residue upstream the óNPSFôsequence in NPAF could yield 11amino acids long peptides such as EFW-NPSF in rat or QFW-NPSF in mice (Roumy et al., 2000; Bonnard et al., 2001; Table 1), ii) cleavage of three amino acid-extended peptides by tripeptidyl peptidases could produce NPFF and the NPAF-derived octapeptide (Perry et al., 1997; Bonnard et al., 2001, 2003) and iii) NPFF and NPSF may represent degradation products of longer peptides (Roumy et al., 2000; Talmont et al., 2010).

\subsubsection{The proNPFF B precursor for RFRPs}

The gene encoding the proNPFF $F_{B}$ precursor contains three coding exons. The alignment of mammalian proNPFF $F_{B}$ precursor polypeptides (189-203 amino acids depending on species) indicates a well conserved position of two distinct RFGR motifs defining RFRP-1 and RFRP-3 peptides C-termini (Hinuma et al., 2000; Fukusumi et al., 2001; Liu et al., 2001). With the exception of Siberian hamster, N-terminal cleavage for RFRP-1 occurs at a single basic R (or K in human) residue. Despite the fact that proNPFF $_{B}$ sequences upstream RFRP-3 are enriched in basic residues, cleavage of mature RFRP-3 peptides occurs at species-specific Arg residues, indicating that primary sequence information but also three-dimensional conformation might control $\mathrm{N}$ terminal cleavage selectivity (Yoshida et al., 2003).

Processing of human, macaque and bovine proNPFF $\mathrm{B}_{\mathrm{B}}$ precursors may produce in addition to RFRP-1 (a LPLRF-amide-containing peptide) and RFRP-3 (a peptide sharing with NPFF a Cterminal PQRF-amide motif), a putative RFRP-2 peptide, with an RS- or RL-amide C-terminal sequence depending on species (Ubuka et al., 2012b). However, the physiological relevance of RFRP-2 in Mammals is unlikely as this peptide has never been isolated from native tissues, lacks 
activity at OT7T022 receptors (Hinuma et al., 2000) and appears to be included in the N-terminal RFRP-3 sequence (Ubuka et al., 2012b). Rodent proNPFF suggesting that it retrogressed during the evolution of Mammals (Yoshida et al., 2003).

Although mammalian RFRPs and avian GnIH precursors exhibit significant conservation, GnIH precursor polypeptide in birds encodes one GnIH and two GnIH related peptides (GnIH-RP-1 and GnIH-RP-2), all harbouring the typical C-terminal LPXRF-amide motif (Liu et al., 2001; Ubuka et al., 2012b). Two of them (GnIH and GnIH-RP-2) were characterized as isolated peptides (Satake et al., 2001). While GnIH-RP-1/RFRP-1 and GnIH/RFRP-2 are encoded at conserved positions in avian and mammalian precursor polypeptides (only RFRP-1 in rodents), the positions of GnIH-RP2 and RFRP-3 were totally different (Ubuka et al., 2012b).

\subsubsection{The PrRP precursor}

In Mammals, the PrRP precursor gene contains two coding exons (Yamada et al., 2001; Lin, 2008). Structurally similar human, bovine, rat and mouse prepro-PrRP proteins (82-98 amino acids depending on species) generate highly conserved mature PrRP peptides (Hinuma et al., 1998; Fukusumi et al., 2006; Lin, 2008). PrRP-20 and PrRP-31 derive from the same precursor protein, with the 20 amino acid-long sequence of PrRP-20 arising from N-terminal cleavage of the larger PrRP-31 peptide at the level of a conserved Arg residue.

\subsubsection{The KiSS-1 protein}

The KiSS-1 gene that contains two coding exons was first identified as a metastasis suppressor gene in human melanoma (Lee et al., 1996). Its primary translation product, the KiSS-1 protein (130-145 amino acids depending on species) display a moderate overall sequence homology in Mammals (Stafford et al., 2002; Terao et al., 2004; Roa \& Tena-Sempere, 2007). Endowed with typical features for a precursor of secreted neuropeptides sharing a RF-amide signature at their Cterminus, the KiSS-1 protein turned out to be the precursor of the entire kisspeptin family (Kotani et al., 2001a; Muir et al., 2001; Ohtaki et al., 2001; Kirby et al., 2010; Pinilla et al., 2012).

Human kisspeptin-54 (metastin), with its sequence surrounded by basic residue pairs, is predicted to result from the cleavage of KiSS-1 by furin or prohormone convertases (Kotani et al., 2001a). The shorter kisspeptin isoforms (Kp-14, -13 and -10), which are all biologically active (Ohtaki et al., 2001), are generated according to processes (differential proteolysis of the KiSS-1 precursor or degradation of the N-terminal part of $\mathrm{Kp}-54$ ) that remain to be clarified as no obvious cleavage sites were identified.

The comparison of predicted sequences for kisspeptins from many mammalian species pointed to a highly conserved kisspeptin-10 sequence and, interestingly, suggested the existence of two 
kisspeptin subfamilies bearing either RF-amide (human and primates) or RY-amide (felines, ruminants, murine, dolphin) C-termini (Roa \& Tena-Sempere, 2007; d'Anglemont de Tassigny \& Colledge, 2010). In rat and mouse, the largest kisspeptin peptide is 52 amino acid long (Kp-52) with Arg-Tyr- $\mathrm{NH}_{2}$ at its $\mathrm{C}$ terminus instead of Arg-Phe- $\mathrm{NH}_{2}$ as in humans (Terao et al., 2004).

\subsubsection{The prepro-QRFP precursor for $Q R F P-26$ and $Q R F P-43$ peptides}

Molecular cloning of the QRFP precursor revealed its presence in all vertebrates and a conserved organization (Chartrel et al., 2011; Ukena et al., 2013). In Mammals, precursor genes were characterized in mouse, rat, bovine and human; three coding exons encode similar (124-136 amino acids long depending on species) prepro-QRFP proteins (Chartrel et al., 2003; Fukusumi et al., 2003; Jiang et al., 2003). They contain a typical RFGR motif for C-terminal cleavage and amidation and several monobasic residues as putative, though non conventional, $\mathrm{N}$-terminal processing sites (Chartrel et al., 2011). Yet, efficient precursor cleavage occurred at a single conserved Lys residue, as assessed from the isolation of mature QRFP-26 (or 26RFa) peptides from frog (Chartrel et al., 2003) and human (Bruzzone et al., 2006) brain. As well, a highly conserved Arg residue, located upstream the QRFP-26 region in the precursor, represents a potential cleavage site for a prohormone convertase, possibly furin (Bruzzone et al., 2006), to generate the N-terminal extended QRFP-43 (or 43RFa) peptide (Bruzzone et al., 2006; Takayasu et al., 2006).

\section{Mammalian RF-amide receptors}

In parallel to the isolation of RF-amide neuropeptides and the characterization of their precursors, five orphan $\mathrm{G}$ protein coupled receptors (GPCRs) were successively identified as the natural targets of endogenous mammalian RF-amides in the late 90ôs and early 2000ôs.

\subsection{The NPFF1 (GPR147) and NPFF2 (GPR74) receptors}

Bonini and collaborators cloned rat and human orthologs of two G protein coupled receptors differing in their primary sequences and their anatomical distribution in rat and human tissues (Bonini et al., 2000). As they exhibited preferential binding affinities and activities, in the (sub)nanomolar concentration range, towards NPFF and some RF-amide derivatives, they were defined as NPFF1 (rBN6 and hBO102) and NPFF2 (rBO119 and hBO89) receptors. Their pharmacological characterization was however incomplete. Following the identification of the proNPFF $F_{B}$ genes, Hinuma and colleagues used a reverse pharmacology approach to provide convincing evidence for RFRPs to be the cognate ligands of the rat and human orphan OT7T022 receptor (Hinuma et al., 
2000). Independently, Elshourbagy and coworkers identified the HLWAR77 receptor as the target of NPFF and NPAF peptides for which it displayed high affinity and potency (Elshourbagy et al., 2000). Meanwhile, Parker and collaborators cloned another putative receptor, called GPR74, structurally related to the neuropeptide Y (NPY) receptor family (Parker et al., 2000).

The systematic comparison of the pharmacological properties of proNPFF $\mathrm{A}^{-}(\mathrm{NPFF}$ and NPAF derivatives) and proNPFF $^{-}$(RFRPs) derived peptides at these diverse RF-amide receptors unambiguously demonstrated the molecular and functional identity of OT7T022 with the NPFF1/GPR147 receptor and of HLWAR77 with the NPFF2/GPR74 one (Liu et al., 2001; Mollereau et al., 2002; Yoshida et al., 2003).

\subsection{The PrRP (PrRP-R, GPR10) receptor}

The orphan GPR10 receptor (Marchese et al., 1995) and its rat ortholog UHR-1 (Welch et al., 1995) turned out to correspond to the human PrRP receptor (first designated as orphan hGR3) targeted by the endogenous prolactin releasing peptides PrRP-20 and PrRP-31 (Hinuma et al., 1998). A highly homologous receptor has been subsequently cloned in mice (Gu et al., 2004).

\subsection{The Kiss1 (GPR54) receptor}

In 1999, Lee and coworkers cloned a rat orphan receptor (named GPR54) encoding for a 396 amino acid polypeptide with significant homology with the galanin receptor within its putative transmembrane (TM) domains (Lee et al., 1999). In 2001, Ohtaki and collaborators found a rat orphan receptor (named rOT7T175), which was nearly identical to GPR54 (Ohtaki et al., 2001). Using its human counterpart hOT7T175 in a reverse pharmacology approach, they identified metastin (kisspeptin-54) as its cognate ligand. Meanwhile, Muir and colleagues cloned the human AXOR12 gene encoding for a 398 amino acid protein also analogous to rat GPR54 (Muir et al., 2001). Using again a reverse pharmacology strategy, they identified a series of peptides derived from the KiSS-1 gene as potent agonists for AXOR12. Thus, OT7T175 and AXOR12 receptors were definitively catalogued as the Kiss1R or GPR54 receptor which is efficiently activated by all kisspeptins, including its shortest kisspeptin-10 member (Kotani et al., 2001a; Muir et al., 2001; Ohtaki et al., 2001).

\subsection{The QRFP (GPR103 or P518) receptor}


Along with the identification of the mouse and human prepro-QRFP gene, Jiang and coworkers (Jiang et al., 2003) identified, among possible RF-amide peptides encoded by the precursor, the P518 peptide (yet QRFP-26) as a putative endogenous ligand for the orphan SP9155 receptor. The QRFP (yet QRFP-43 or 43RFa) peptide, generated by the same precursor protein, was also isolated and found to be a high affinity and potent agonist at the human orphan AQ27 receptor (Fukusumi et al., 2003). Altogether, both SP9155 and AQ27 were identical to the orphan GPR103 receptor previously reported in cattle (Lee et al., 2001). To note that the IUPHAR/BPS Guide to Pharmacology (http://www.guidetopharmacology.org) adopted the P518 receptor terminology according to the endogenous P518 peptide (or 26RFa) isolated by Jiang and colleagues (Jiang et al., 2003).

Humans have only one receptor for QRFP peptides while rodents have two receptor isoforms. Indeed, two orthologs of human GPR103 have been found in mouse and rat genomes and designated as GPR103A and GPR103B (Takayasu et al., 2006) and as QRFP-r1 and QRFP-r2 (Kampe et al., 2006), respectively. In this review, we will use the terminology QRFP-R1 and QRFP-R2 for both mouse and rat receptor subtypes.

\subsection{Structural information}

\subsubsection{The NPFF1 (GPR147) and NPFF2 (GPR74) receptors}

In humans, the NPFF1R and NPFF2R genes are located on chromosomes 10 (locus 10q21-22) and 4 (locus 4q21), respectively. Both contain 4 coding exons; no (NPFF1R) or up to three (NPFF2R) splice variants have been identified (further information, including other mammalian species, is available on IUPHAR and NCBI websites). As deduced from their cDNAs, rat and human NPFF1 receptors are proteins of 432 and 430 amino acids, respectively, which have $86 \%$ identity with each other (Bonini et al., 2000; Hinuma et al., 2000). Rat and human NPFF2 receptors (417 and 420 amino acids, respectively) share $78 \%$ identity and are nearly 50\% identical to rat and human NPFF1 (Bonini et al., 2000; Elshourbagy et al., 2000). Receptor- and species-linked sequence divergences essentially concern the receptor N-terminus and the second extracellular loop (domains that might be critical for ligand recognition and binding selectivity) as well as the third intracellular loop and the C-terminal tail known to interact with various signalling partners.

Comparing the primary sequence of human NPFF1 with that of other 7TMRs (for which ligands have been identified) showed high homology (31-37\% identity) with human orexin receptors 1 and 2, NPY1, 2 and 4 receptor subtypes, cholecystokinin (CCK) receptor A and the prolactin-releasing hormone (PRL) receptor (Bonini et al., 2000; Hinuma et al., 2000). The human NPFF2/GPR74 
receptor has been also shown to exhibit high amino acid identity (33\%) to the human NPY2 receptor subtype (Parker et al., 2000).

The existence of variants of the NPFF2 receptor has been reported in human and mouse (Cikos et al., 1999; Parker et al., 2000; Laemmle et al., 2003; Anko et al., 2006). The first description of a novel putative GPCR, termed NPGPR (Cikos et al., 1999), received poor attention as it was rapidly coined as an aberrant variant of the human NPFF2 receptor with an extended N-terminus (Elshourbagy et al., 2000) and a cloning artefact (Parker et al., 2000). Taking advantage of the determination of the exon-intron structure of the GPR74 gene (Parker et al., 2000), Laemmle and colleagues (Laemmle et al., 2003) identified three different forms of the human NPFF2 receptor resulting from alternative exon splicing : a full length NPGPR, most identical to the sequence published by Cikos and collaborators (Cikos et al., 1999), differs from HLWAR77 by an additional exon (exon 1) encoding 102 additional N-terminal residues. The GPR74 sequence differs from hNPFF2R both at 5-Óand 3-óends, hence it contains 3 additional aa and lacks 15 aa at the receptor $\mathrm{N}$ - and C-termini, respectively. Finally, a very short form of NPGPR, lacking exon 2 and encoding for a putative soluble protein of 132 amino acids, was also identified. Both NPGPR transcripts differed in their distribution in human tissues while binding studies indicated that the affinity of the full-length NPGPR for NPFF was nearly 100-fold lower than that of NPFF2 (Laemmle et al., 2003). NPGPR forms were not found in mouse unlike NPFF2 and GPR74 which were considered as alternatively spliced receptor variants (Anko et al., 2006).

\subsubsection{The PrRP (GPR10) receptor}

In humans, the PrRP-R gene is located on chromosome 10 (locus 10q26.13). It contains three coding exons that give rise to two splice variants (further information, including other mammalian species, is available on IUPHAR and NCBI websites). Human, rat and mouse PrRP receptors are 370 amino acid long proteins with a sequence identity close to $90 \%$ ( $\mathrm{Gu}$ et al., 2004). They are closely related to the Neuropeptide Y receptor family (31\% overall identity and $46 \%$ identity in TM domains) but they do not bind NPY-related peptides (Marchese et al., 1995).

\subsubsection{The Kiss1R (GPR54) receptor}

In humans, the Kiss1R gene is located on chromosome 19 (locus 19p13.3). It contains five coding exons with no known splice variants (further information, including other mammalian species, is available on IUPHAR and NCBI websites). Rat (396 amino acids) and human (398 amino acids) kisspeptin receptor proteins share $85 \%$ sequence identity (98\% identity in TM domains) whereas mouse and human proteins display $82 \%$ identity (Kirby et al., 2010). The N- 
terminus, the third extracellular loop and the C-terminal intracellular domain of rat and human receptor orthologs are less conserved (Kotani et al., 2001a).

The human Kiss1R displays a significant homology with the human GalR1, GalR2, and GalR3 (Muir et al., 2001; Ohtaki et al., 2001) although there is some discrepancy whether highest sequence identity is to GalR3 (30\%; Muir et al., 2001) or to GalR1 (45\%; Kirby et al., 2010). It is clear however that Kiss1R does not interact with galanin (Lee et al., 1999).

\subsubsection{The QRFP (QRFP-R, GPR103 or P518) receptor}

In humans, the QRFP-R gene is located on chromosome 4 (locus 4q27). It contains eight coding exons with multiple possibilities of alternative splicing (further information, including other mammalian species, is available on IUPHAR and NCBI websites). As inferred from cDNA analyses, the mammalian QRFP receptor proteins vary in amino-acid (a.a.) length and content depending on their human (431 a.a.), mouse (GPR103A : 433 a.a.; GPR103B : 425 a.a.) or rat (QRFP-r1 : 433 a.a.; QRFP-r2 : 427 a.a.) origin (Fukusumi et al., 2003; Kampe et al., 2006; Takayasu et al., 2006). Rodent GPR103 isoforms share 75\% and 78\% identity in mouse and rat, respectively. Human QRFP-R exhibits a slightly higher homology with GPR103A (85\% identity) and QRFP-r1 (84\% identity) subtypes, as compared to GPR103B (79\% identity) or QRFP-r2 (82\%) receptors (Kampe et al., 2006; Takayasu et al., 2006).

QRFP receptors display some sequence homology with several GPCRs, including the orexin, galanin GalR1, NPY2 and CCK receptors, but NPFF1 and NPFF2 receptors are their closest known parents, with amino acid identity close to 50\% (Jiang et al., 2003; Chartrel et al., 2011).

\section{RF-amides in vitro pharmacology : Ligand-receptor binding properties}

Binding properties of RF-amide receptors are essentially from standard radioligand binding studies using iodine-labelled peptides and membrane preparations from brain tissues or from heterologous cells transiently or stably expressing recombinant RF-amide receptors. Owing to their high specific radioactivity, $\left[{ }^{125} \mathrm{I}\right]$-labelled $\mathrm{RF}$-amide peptides allow the detection of minute amounts of receptors and the reliable characterization of binding sites endowed with nano or subnanomolar affinity properties. Specific binding was usually defined as part of total binding displaceable by micromolar concentrations of unlabelled peptides. Most studies are performed under equilibrium binding conditions in order to determine receptor affinities (apparent equilibrium dissociation constants) for $\left[{ }^{125} \mathrm{I}\right]$-tracers ( $\mathrm{K}_{\mathrm{d}}$ values) or unlabelled compounds ( $\mathrm{K}_{\mathrm{i}}$ values) from saturation or competition experiments, respectively. 
An overview of the characteristics of available tracers and of the binding affinity properties of the five RF-amide receptors for a representative set of peptides is presented in Tables 2 and 3. For a sake of clarity, only $\mathrm{K}_{\mathrm{d}}$ or $\mathrm{K}_{\mathrm{i}}$ values determined on recombinant RF-amide receptor transfected in heterologous cell lines are listed.

\subsection{Radiolabelled tracers for RF-amide receptors}

\subsubsection{NPFF1R (GPR147) and NPFF2R (GPR74) radioligands}

A iodinated analog of NPFF (with Phe ${ }^{1}$ substituted for a Tyr residue), known as $\left[{ }^{125}\right.$ I]Tyr-NPFF or $\left[{ }^{125} \mathrm{I}\right] \mathrm{Y} 8 \mathrm{Fa}$, was first introduced to characterize putative binding sites for this morphine modulating peptide (Allard et al., 1989). Thereafter, [D.Tyr ${ }^{1}$, (NMe)Phe ${ }^{3}$ NPFF (with substitution of L-Phe ${ }^{1}$ for D-Tyr and N-methylation of the second peptidic bond) was selected out of a series of NPFF analogs for its high affinity and greater stability than Y8Fa (Gicquel et al., 1992, 1994). Its radio-iodinated derivative (Devillers et al., 1994), referred to as $\left[{ }^{125} \mathrm{I}\right]-\left[\mathrm{D} . \mathrm{Tyr}^{1}\right.$, (NMe)Phe $\left.{ }^{3}\right] \mathrm{NPFF}$, $\left[{ }^{125} \mathrm{I}\right]-(1 \mathrm{DMe}) \mathrm{NPFF},\left[{ }^{125} \mathrm{I}\right]-(1 \mathrm{DMe}) \mathrm{Y} 8 \mathrm{Fa}$ or even $\left[{ }^{125} \mathrm{I}\right]-1 \mathrm{DMe}$, became a popular tracer for NPFF receptors, especially in autoradiographic studies (Gouarderes et al., 1997, 2000). [ $\left.{ }^{125} \mathrm{I}\right] \mathrm{Y} 8 \mathrm{Fa}$ and $\left[{ }^{125} \mathrm{I}\right]-(1 \mathrm{DMe}) \mathrm{Y} 8 \mathrm{Fa}$ display comparable affinities for both NPFF1 and NPFF2 receptors (Table 2).

The identification of $\mathrm{NPFF}_{\mathrm{A}}$ and $\mathrm{NPFF}_{\mathrm{B}}$ precursors and of two NPFF receptor subtypes prompted the search for R1- and R2-selective radioligands. Iodinated derivatives of RFRP-1 (YANLPLRFa) and RFRP-3 (YVPNLPQRFa) peptides, respectively referred to as [ $\left.{ }^{125} \mathrm{I}\right]-\mathrm{Tyr}-$ hNPSF (Liu et al., 2001) and [ $\left.{ }^{125} \mathrm{I}\right]$-Tyr-hRFRP-3 (Hinuma et al., 2000) or [ $\left.{ }^{125} \mathrm{I}\right]$-YVP (Gouarderes et al., 2002), were introduced as NPFF1-selective tracers. Conversely, $\left[{ }^{125} \mathrm{I}\right]$-EYWSLAAPQRFa or $\left[{ }^{125} \mathrm{I}\right]-\mathrm{EYF}$, derived from a peptide present in the rat $\mathrm{NPFF}_{\mathrm{A}}$ precursor (Table 1), was characterized as a high affinity NPFF2-selective probe (Gouarderes et al., 2001).

$\left[{ }^{3} \mathrm{H}\right]$-FFRFa, a tritiated analog of a high affinity tetrapeptide at endogenous NPFF receptors (Payza et al., 1993) has been used in some binding studies (Gealageas et al., 2012; Elhabazi et al., 2013; Bihel et al., 2015) although it exhibits a poor NPFF1/2 binding selectivity (Table 2). More recently, $\left[{ }^{3} \mathrm{H}\right]$-hRFRP-3 and $\left[{ }^{3} \mathrm{H}\right]$-EYF have been obtained by tritiation of the corresponding dehydro-Leu peptide precursors and characterized as interesting alternatives to radio-iodinated compounds (Talmont et al., 2009). More anecdotal probes were also reported such as rhodaminelabelled NPFF (TAMRA-NPFF) that might be useful in fluorescence polarization-based binding assays (Do et al., 2004).

The data presented in Table 2 deserve additional comments. Indeed, most saturation binding studies performed on membrane preparations from HEK or CHO cells transfected with individual receptors indicated an apparent homogenous population of high affinity binding sites. $\mathrm{K}_{\mathrm{d}}$ values for 
most radioligands are within the $0.1-1 \mathrm{nM}$ concentration range indicating a very high affinity of all these radiolabelled agonists for NPFF1 and NPFF2 receptors. Similar saturation studies, mostly focusing on NPFF2 receptors in native tissues, were performed on membrane preparations of rat spinal cord using $\left[{ }^{125} \mathrm{I}\right] \mathrm{Y} 8 \mathrm{Fa}\left(\mathrm{K}_{\mathrm{d}}: 0.09 \mathrm{nM}\right.$; Allard et al., 1989) or $\left[{ }^{125} \mathrm{I}\right]-(1 \mathrm{DMe}) \mathrm{NPFF}\left(\mathrm{K}_{\mathrm{d}}: 0.07\right.$ $\mathrm{nM}$; Devillers et al., 1994) or of olfactory bulb using [ $\left.{ }^{3} \mathrm{H}\right]-\mathrm{EYF}\left(\mathrm{K}_{\mathrm{d}}: 0.4 \mathrm{nM}\right.$; Talmont et al., 2010).

$\mathrm{K}_{\mathrm{d}}$ values for $\left[{ }^{125} \mathrm{I}\right]-(1 \mathrm{DMe}) \mathrm{NPFF}\left(0.1 \mathrm{nM}\right.$; Gouarderes et al., 1997) and [ $\left.{ }^{125} \mathrm{I}\right]-\mathrm{EYF}(0.02-0.04$ $\mathrm{nM}$; Gouarderes et al., 2001) were also determined from quantitative autoradiographic binding studies on rat and mouse brain tissue sections. It remains that true saturation was rarely achieved using radio-iodinated tracers (probably due to cost-effective manipulations and prohibitive radioactivity levels at high concentration) and that the maximal density in receptor sites $\left(\mathrm{B}_{\max }\right)$ was in general extrapolated. Moreover, when compared to their iodinated analogs (Table 2), $\left[{ }^{3} \mathrm{H}\right]-$ hRFRP-3 and $\left[{ }^{3} \mathrm{H}\right]$-EYF tracers displayed a 10- to 20-fold lower affinity for NPFF1 and NPFF2 receptors, respectively (Talmont et al., 2009, 2010). As well, $\left[{ }^{3} \mathrm{H}\right]-\mathrm{EYF}$ was reported to label a much greater amount of NPFF2 sites than $\left[{ }^{125} \mathrm{I}\right]-\mathrm{EYF}$ (Talmont et al., 2009). These observations tend to indicate that iodinated peptides are probably used under experimental conditions favouring the selection of a high affinity receptor site subpopulation (Zajac \& Roques, 1989; Talmont et al., 2009).

\subsubsection{PrRP-R, Kiss $1 R$ and $Q R F P-R$ radioligands}

The binding properties of these receptors have been studied using a limited set of radio-iodinated peptides. [ $\left.{ }^{125} \mathrm{I}\right]$-hPrRP-20 (Langmead et al., 2000) and $\left[{ }^{125} \mathrm{I}\right]-\mathrm{PrRP}-31$ of human (Roland et al., 1999), bovine (Hinuma et al., 1998) or rat (Maixnerova et al., 2011) origin were obtained through introduction of a ${ }^{125} \mathrm{I}$ atom at the level of the highly conserved Tyr residue in the PrRP sequence (Table 1). They display similar $K_{d}$ values at the PrRP receptor (Table 3). Noteworthy, the introduction of the long lifetime europium lanthanide to a Lys residue added to the N-terminus of hPrRP-31 led to Eu-(Lys)hPrRP-31, an original tracer prone to time-resolved fluorescence measurements (Boyle et al., 2005).

Similarly to the labelling of PrRPs, the single conserved Tyr residue in human kisspeptins was iodinated to give $\left[{ }^{125} \mathrm{I}\right]$-Kisspeptin-10 (Kotani et al., 2001a), $\left[{ }^{125} \mathrm{I}\right]$-Kisspeptin-13 (Mead et al., 2007) and $\left[{ }^{125} \mathrm{I}\right]$-Kisspeptin-15 (Ohtaki et al., 2001) tracers. All of them display very similar binding affinities at the Kiss1 receptor (Table 3). Iodinated kisspeptin-54 was essentially used in radioimmunoassays (Dhillo et al., 2005).

To our knowledge, binding studies performed on QRFP receptors are few and only $\left[{ }^{125} \mathrm{I}\right]-\mathrm{QRFP}-$ 43 has been used as a radiotracer (Fukusumi et al., 2003; Takayasu et al., 2006; Elhabazi et al., 2013). 


\subsection{Binding affinity properties of RF-amide receptors for their endogenous peptides}

Values reported in Tables 2 and 3 are from competition studies performed on membranes preparations from $\mathrm{CHO}$ or HEK cells expressing recombinant receptors, using fixed concentrations of tracers (usually close or below their respective $K_{d}$ values). $K_{i}$ values are taken from a selection of papers that examined the binding affinities of the five human RF-amide receptors for different RFamide peptides.

\subsubsection{NPFF1 (GPR147) and NPFF2 (GPR74) receptors}

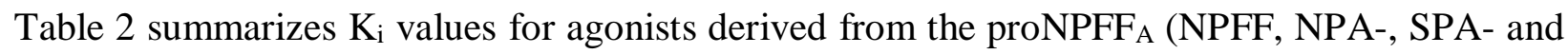
SQA-NPFF, and NPAF peptides from various species including the óNPSFôoctapeptide) and the proNPFF $_{B}$ (RFRP-1 and RFRP-3) precursors as well as for other RF-amide peptides such as FMRFa, PQRFa and the frog pancreatic peptide. This list also includes $\mathrm{K}_{\mathrm{i}}$ values for unlabelled tracer analogs regarded here as competitors.

RFRP-1 and RFRP-3 peptides display highest affinity for human NPFF1 receptors $\left(\mathrm{K}_{\mathrm{i}}\right.$ range : 0.4-2.7 $\mathrm{nM}$ ), followed by NPFF derivatives ( $\mathrm{K}_{\mathrm{i}}$ range : $\left.2.4-15 \mathrm{nM}\right)$ and finally by the NPAF peptide group $\left(\mathrm{K}_{\mathrm{i}}\right.$ range : 10-50 $\left.\mathrm{nM}\right)$. Affinity ranking of the same series of compounds at the human NPFF2 receptor indicates a very modest (if no) preference for NPFF-type agonists $\left(K_{i}\right.$ range : 0.03$1.1 \mathrm{nM}$ ) over NPAF-type peptides ( $\mathrm{K}_{\mathrm{i}}$ range : 0.15-1.8 $\mathrm{nM}$ ), with exception of the octapeptide NPSF $\left(\mathrm{K}_{\mathrm{i}}:\right.$ 12-37 $\left.\mathrm{nM}\right)$, and a lower affinity for RFRP-1 and RFRP-3 ( $\mathrm{K}_{\mathrm{i}}$ range : 3-150 $\left.\mathrm{nM}\right)$. Thus, RF-amide peptides resulting from the cleavage of $\operatorname{proNPFF}_{\mathrm{B}}$ preferentially bind the NPFF1 receptor while the reverse is true for $\operatorname{proNPFF}_{\mathrm{A}}$-derived peptides at the NPFF2 receptor.

As most $\mathrm{K}_{\mathrm{i}}$ values (as afforded from various studies using identical or distinct radioligands) are in overall agreement, one may assume that RF-amide peptides compete with tracers for binding to an identical and homogenous population of NPFF1 or NPFF2 receptor sites. Some differences have been noted depending on the type of radioligand (Table 2), such as a 5- to 6-fold lower binding affinity of peptides when assessed using [ $\left.{ }^{3} \mathrm{H}\right]$-hRFRP-3 (Talmont et al., 2009) or a 10- to 20- higher affinity of several peptides when using $\left[{ }^{3} \mathrm{H}\right]-$ FFRF (Elhabazi et al., 2013) instead of $\left[{ }^{125} \mathrm{I}\right]-\mathrm{YVP}$ at the NPFF1 receptor. Affinity rank order was however preserved.

Binding selectivity of RF-amide peptides at NPFF1 and NPFF2 receptors is rather difficult to express in absolute quantitative terms as it hardly depends on the radioligand pairs which are considered (Table 2). Taking mean $\mathrm{K}_{\mathrm{i}}$ values for the whole set of available data for each peptide, selectivity profiles for NPFF1- or NPFF2-preferring compounds may be proposed as follows. hRFRP-1 (37 aa), hRFRP-1 (12aa), FMRFa, hY-RFRP-3 (or YVP; 9aa) and hRFRP-3 (8aa) display 
a 2-, 3-, 5-, 20- and 50-fold higher affinity for NPFF1R than for NPFF2R, respectively. Conversely, while the octapeptides (NPFF, 1-DMe and NPSF) display a very poor selectivity towards NPFF2 receptors (close to 2-fold), the undecapeptides derived from the $\mathrm{NPFF}_{\mathrm{A}}$ precursor exhibit a much greater preference for NPFF2 receptors. Indeed, their selectivity indexes rise from 30, 40 to 130 for human SQA-, mouse SPA- and rat NPA-NPFF derivatives, and from 60, 85 to 160 for EYW- (or EYF), mouse QFW- and rat EFW-NPAF derivatives, respectively.

To note that the frog pancreatic peptide (fPP) is the most NPFF2 selective peptide (Table 2). It proved to be an efficient NPFF2R blocker to reinforce the NPFF1 nature of $\left[{ }^{125} \mathrm{I}\right]-(1 \mathrm{DMe}) \mathrm{NPFF}$ (Bonini et al., 2000) or [ $\left.{ }^{125} \mathrm{I}\right]$-YVP (Gouarderes et al., 2002, 2004b) binding on native rat tissues.

Overall, ligand binding properties of NPFF receptors are essentially from studies performed on membrane preparations from recombinant HEK (Bonini et al., 2000; Gouarderes et al., 2002) or CHO cells (Kotani et al., 2001b; Mollereau et al., 2002; Gouarderes et al., 2002; Engstrom et al., 2003; Yoshida et al., 2003; Gouarderes et al., 2007; Talmont et al., 2010), with special focus on receptors of human origin. Given the low density of receptor sites in native tissues and possible mismatches due to co-expression of various receptor subtypes and to low radioligand binding selectivity, only a few experiments addressed the pharmacological properties of endogenous NPFF receptors. It remains that binding studies performed on rat spinal cord slices (Roumy et al., 2000; Gouarderes et al., 2001; Mazarguil et al., 2001) or membrane preparations (Gouarderes et al., 2002; Engstrom et al., 2003) and on mouse olfactory bulb membranes (Gherardi \& Zajac, Peptides 1997; Gouarderes et al., 2001;Talmont et al., 2010) adequately reported on ligand binding properties of endogenous NPFF2R. Indeed, these CNS areas are highly enriched in this receptor subtype (Bonini et al., 2000; Gouarderes et al., 2001, 2002, 2004a), are easy to dissect and provide sufficient material. Although the overall binding profiles of human and rodent NPFF2 receptors were comparable, the recurrent observation of a slightly higher affinity of rodent receptors for most RFamide peptides was further investigated in a systematic comparative study by Talmont and coworkers (2010). The octapeptides NPSF and NPVF (or RFRP-3), respectively derived from the pro-NPFF ${ }_{A}$ and pro-NPFF ${ }_{B}$ precursors, as well as the non selective NPFFR antagonist RF9, were found to display a tenfold higher affinity for the mouse NPFF2 receptor than for the human one. Thus, species-related differences in NPFF2R binding properties might have a repercussion on the evaluation of drug pharmacological properties in vivo.

\subsection{2. $\operatorname{PrRP}(G P R 10)$, Kiss $1 R(G P R 54)$ and $Q R F P(G P R 103)$ receptors}

In contrast to the fairly high affinities of most RF-amides at both NPFF receptors (see below), the PrRP, the Kiss1R and QRFP receptors are very strong peptide discriminators (Table 3). Subnanomolar binding affinities and functional potencies are exclusively limited to their 
endogenous ligands while all other peptides display no significant interaction, even in the micromolar range. Noteworthy, the existence of receptor subtypes in mouse and rat is not associated with appreciable differences in their pharmacological properties (Kampe et al., 2006; Takayasu et al., 2006).

\subsection{Promiscuous binding properties of NPFF1 and NPFF2 receptors}

Several independent observations suggested the possibility for PrRPs (Engstrom et al., 2003; Ma et al., 2009), Kisspeptins (Lyubimov et al., 2010) and QRFPs (Bruzzone et al., 2007) to signal through NPFF receptors in vivo, in addition to their classical effects mediated by their respective PrRP, KiSS-1 and QRFP receptors. As an example, the pivotal role of NPFF receptors in the hyperalgesic and anti-morphine effects of PrRP-20, kisspeptin-10 and QRFP-26 was demonstrated in a systematic in vivo study, taking advantage of the selective NPFF receptor antagonist RF9 (Elhabazi et al., 2013).

More direct evidence for a low level of discrimination of NPFF1 and NPFF2 receptors towards most RF-amide peptides was provided by a set of in vitro binding and functional studies performed on heterologous cell lines expressing either NPFF receptor.

Data summarized in Table 3 indicate that PrRPs (Engstrom et al., 2003; Gouarderes et al., 2007; Elhabazi et al., 2013), Kisspeptins (Oishi et al., 2011; Elhabazi et al., 2013) and QRFPs (Gouarderes et al., 2007; Elhabazi et al., 2013) bind with nanomolar affinity to both NPFF1 and NPFF2 receptors. PrRPs and QRFPs exhibit a moderate NPFF2 selectivity whereas Kisspeptins exhibit a slight preference for NPFF1 receptors. The affinity of kisspeptins for the NPFF1 receptor was nearly equivalent to that of RFRPs while prolactin releasing peptides bound in a concentration range similar to that of proNPFF$_{\mathrm{A}}$-derived peptides to NPFF2 receptors.

Functional studies (Table 3) also indicated that all RF-amide peptides were able to mediate a typical $\mathrm{G}_{\mathrm{i} / \mathrm{o}}$ response through the stimulation of NPFF1 and NPFF2 receptors, i.e. a concentrationdependent inhibition of forskolin-induced cAMP accumulation. In general, PrRPs, kisspeptins and QRFPs potencies were lower than those of typical NPFF agonists. It remains that PrRPs at NPFF2R as well as kisspeptin-10 and kisspeptin-13 at both NPFF receptors promoted significant responses in the submicromolar concentration range, as did several $\operatorname{proNPFF}_{\mathrm{A}}$ and $\operatorname{proNPFF}_{\mathrm{B}}$-derived peptides at NPFF1 and NPFF2 receptors, respectively.

Thus, in contrast with the three other RF-amide receptors, NPFF1 and NPFF2 receptors exhibit a complex pharmacology, at least in vitro, as they are targeted by most RF-amide peptides. Only careful evaluation of the nature of the receptors involved in PrRPs, Kisspeptins and QRFPs in vivo 
effects, using for example highly selective antagonists or knockout mice for the different RF-amide receptors, will avoid misleading conclusions especially in tissues enriched in NPFF receptors.

\section{RF-amides in vitro pharmacology : Intracellular signalling pathways}

Following their discovery, RF-amide receptors have been rapidly assigned to G-protein coupled receptors according to their typical 7TM signatures, their modulation by guanine nucleotides and, more recently, for their ability to promote the binding of non hydrolysable GTP analogues following agonist stimulation. Several studies have been performed to characterize the nature of the $\mathrm{G}$ proteins involved in RF-amide receptor function as follows.

\subsection{NPFF1R and NPFF2R signalling pathways}

$\mathrm{G} \alpha_{\mathrm{i} / \mathrm{o}}$ coupling of NPFF1 and NPFF2 receptors is now well established. It has been verified using various procedures aiming at quantifying a dose-dependent inhibition of forskolin-stimulated accumulation of cAMP by RF-amide peptides in various heterologous cell lines (CHO, COS, HEK, SH-SY57) expressing either NPFF1 (Hinuma et al., 2000; Fukusumi et al., 2001; Liu et al., 2001; Mollereau et al., 2002; Kersante et al., 2006; Oishi et al., 2011; Elhabazi et al., 2013) or NPFF2 (Elshourbagy et al., 2000; Liu et al., 2001; Mollereau et al., 2002; Yoshida et al., 2003; Talmont et al., 2010; Oishi et al., 2011; Elhabazi et al., 2013) receptors. These cAMP responses were typically abolished by cell pre-treatment with the pertussis toxin PTX. All RF-amide peptides had a similar maximal efficacy and behaved as full agonists (Mollereau et al., 2002).

Table 3 summarizes potencies values of the five groups of RF-amide peptides for inhibiting cAMP production $\left(\mathrm{IC}_{50}\right)$ and allows their comparison with binding affinity constants $\left(\mathrm{K}_{\mathrm{i}}\right)$. Listing lower and upper limits for $\mathrm{IC}_{50}$ values reported in the literature allows to appreciate great variations for some agonists that may be explained by differences in receptor expression levels or assay conditions.

ProNPFF $_{\mathrm{A}^{-}}$and $\operatorname{proNPFF}_{\mathrm{B}}$-derived peptides are the most potent ones at NPFF2 and NPFF1 receptors, respectively, in agreement with their binding affinity properties. The NPFF1 receptor appears a much better peptide discriminator in terms of functional potency than binding affinity : most RF-amide peptides exhibit a nanomolar affinity while PrRPs, Kisspeptins and QRFPs show only very modest potencies in the low micromolar range. Such an exclusion of surrogate agonists for promoting a robust cAMP response was less marked with the NPFF2 receptor as RFRPs, PrRPs and Kisspeptins were active in the submicromolar range. Another interesting observation (Table 3) is the apparent lack of concordance between affinity and functional parameters. This may arise from 
assay conditions (radioligand nature, buffer composition, incubation time) and functional contexts (recombinant cells, receptor expression levels, modified G-proteins and/or reporter systems) that probably do not reproduce native physiological ones. However, one cannot exclude that it may also meet predictions of the so-called ligand paradox (Rosenkilde \& Schwartz, 2000; Kenakin \& Onaran, 2002), revisited into the more recent concept of biased agonism (Galandrin et al., 2007; Kenakin \& Christopoulos 2013; Wisler et al., 2014). Indeed, they both describe the $K_{i}$ value as a macroscopic binding affinity constant for an ensemble of possible receptor conformations whereas potency is linked to the ability of an agonist to stabilize the active receptor state (s) relevant to the type of response which is monitored.

Except the mention of a small NPFF-induced increase in inositol phosphate release in NPFF1transfected $\mathrm{CHO}$ cells, which most likely resulted from activation of the $\mathrm{G}_{\mathrm{q}} / \mathrm{PLC}$ pathway secondary to that of PTX-sensitive G proteins (Bonini et al., 2000), no mobilization of intracellular calcium nor release of arachidonic acid metabolites could be detected upon stimulation of NPFF receptors (Hinuma et al., 2000). Conversely, several studies took advantage of the existence of

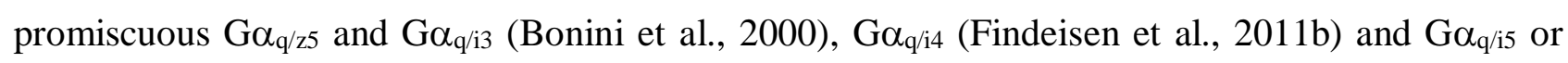
$\mathrm{G} \alpha_{\mathrm{q} / 05}$ (Elshourbagy et al., 2000; Liu et al., 2001) proteins - with $\mathrm{G} \alpha_{\mathrm{i}}, \mathrm{G} \alpha_{\mathrm{o}}$ and $\mathrm{G} \alpha_{\mathrm{z}}$ belonging to the inhibitory class of G proteins (Wettschureck \& Offermanns 2005)- to redirect NPFF receptors towards a robust and easy to monitor intracellular calcium release in response to RF-amide peptides. Similarly, the human $G \alpha_{16}$ protein and its murine counterpart $G \alpha_{15}$ - which belong to the $\mathrm{G} \alpha_{\mathrm{q}}$ class of subunits (Wettschureck \& Offermanns 2005)- were employed to artificially couple NPFF1R (Kim et al., 2015) and NPFF2R (Kotani et al., 2001b) to phospholipase C activation.

A few data in the literature suggested a possible coupling between NPFF receptors and Gas. NPFF and 1-DMe were reported to stimulate, though at a very high concentration, adenylate cyclase in mouse olfactory bulb and spinal cord membranes (Gherardi \& Zajac, 1997). In isolated rat dorsal raphe neurons which express essentially NPFF2 receptors (Gouarderes et al., 2002), NPFF was found to partially block the inhibition exerted by nociceptin on $\left[\mathrm{Ca}^{2+}\right]_{i}$ transients triggered by depolarization (Roumy \& Zajac, 1999); such an effect was prevented by the cholera toxin CTX, indicating that these receptors may couple to stimulatory $G_{s}$ proteins (Roumy \& Zajac, 2001). As well, co-transfection of NPFF1 receptors with the $\mathrm{G} \alpha_{\mathrm{q} / \mathrm{s}}$ chimera allowed NPFF to promote a weak but significant intracellular calcium mobilization in COS cells (Bonini et al., 2000). No responses were observed with the NPFF2 receptor, suggesting again coupling differences between the two NPFF receptors.

It is now well admitted that GPCRs may couple with a variety of G proteins, or even bypass these canonical partners, to initiate an array of intracellular signalling cascades (Defea, 2008; Maurice et al., 2011). The selection of particular pathways is primarily dependent on the agonist 
nature, a phenomenon often referred to as biased agonism (Galandrin et al., 2007; Kenakin \& Christopoulos, 2013; Wisler et al., 2014). In order to investigate the repertoire of endogenous Gproteins involved in NPFF-mediated signal transduction, several synthetic peptides corresponding to the last ten carboxy-terminal residues of various $\mathrm{G} \alpha$ subunits were tested for their ability to alter NPFF receptor binding or function. Following their delivery in SH-SY5Y cells stably expressing the NPFF2 receptor, the $G \alpha_{i 1,2}, G \alpha_{o}$ and $G \alpha_{i 3}$ peptides inhibited specific [ $\left.{ }^{125} \mathrm{I}\right]-\mathrm{EYF}$ binding to various extents, probably by promoting R-G uncoupling (Mollereau et al., 2005a). Surprisingly, the $\mathrm{G} \alpha_{\mathrm{s}}$ peptide was as efficient as $\mathrm{G} \alpha_{\mathrm{i} 1,2}$ in reducing $\left[{ }^{125} \mathrm{I}\right]-\mathrm{EYF}$ binding and was found to potentiate the anti-opioid effect of 1-DMe in $\mathrm{Ca}^{2+}$ conductance measurements. Using the $\left[{ }^{35} \mathrm{~S}\right]-\mathrm{GTP} \gamma \mathrm{S}$ binding experimental paradigm and various blocker peptides, Gouarderes and colleagues showed that $\mathrm{G \alpha}_{\mathrm{i} 3}$ and $\mathrm{G} \alpha_{\mathrm{s}}$ are the main transducers for NPFF1 receptors in CHO cells while NPFF2 may couple to $\mathrm{G} \alpha_{\mathrm{i} 2}, \mathrm{G} \alpha_{\mathrm{i} 3}, \mathrm{G} \alpha_{\mathrm{o}}$ and $\mathrm{G} \alpha_{\mathrm{s}}$ (Gouarderes et al., 2007).

Many GPCRs exploit the release of the G $\beta \gamma$ heterodimer, consecutive to G protein activation (essentially the $\mathrm{G}_{\mathrm{i} / \mathrm{o}}$ class), to enrich their signalling repertoire (Clapham \& Neer 1997; Wettschureck \& Offermanns 2005). Consistent with this observation, short application (15-30 sec) of various NPFFR agonists was found to inhibit voltage-gated (N-type) $\mathrm{Ca}^{2+}$ channels in dissociated DRG neurons (Roumy \& Zajac 1996; Gelot et al., 1998) and in SH-SY5Y neuroblastoma cells transfected with NPFF1 (Kersanté et al., 2006) or NPFF2 (Mollereau et al., 2007) receptors and to enhance $\mathrm{G}_{\mathrm{q}}$-triggered $\mathrm{IP}_{3}$-gated $\mathrm{Ca}^{2+}$ channel activity in NPFF1R- (Kersanté et al., 2006) and NPFF2R- (Mollereau et al., 2005a) expressing SH-SY5Y cells. Both responses, which were sensitive to PTX treatment, most likely involved the mediation of $G \beta \gamma$ subunits released from $\mathrm{G}_{\mathrm{i} / \mathrm{o}}$ proteins. Indeed, inhibition of $\left[\mathrm{Ca}^{2+}\right]_{\mathrm{i}}$ transients is known to rely on direct $\mathrm{G} \beta \gamma$ binding to the $\alpha_{1}$ subunit of Cav2 calcium channels (Clapham \& Neer 1997; Zamponi \& Currie 2013) whereas coincident signalling between $\mathrm{G}_{\mathrm{i} / \mathrm{o}}$ and $\mathrm{G}_{\mathrm{q}}$ coupled receptors arises from a physical interaction between G $\beta \gamma$ and PLC, leading to a potentiation of Gq-triggered $\mathrm{Ca}^{2+}$ release from intracellular stores (Philip et al., 2010; Lyon \& Tesmer 2013).

Interestingly, the attenuation of $\left[\mathrm{Ca}^{2+}\right]_{i}$ transients in response to acute NPFFR activation vanished with prolonged agonist pre-incubation time (20-30 $\mathrm{min})$, probably due to receptor desensitization (Kersanté et al., 2006; Mollereau et al., 2007). Such a condition (lack of direct effect of NPFF derivatives on the magnitude of $\left[\mathrm{Ca}^{2+}\right]_{i}$ rise evoked by cell depolarization) was selected in a number of studies to evidence the anti-opioid effects of NPFF receptors, in recombinant cells and in isolated neurons (Mollereau et al., 2005b; Mouledous et al., 2010b, for reviews)

Consistent with a recurrent observation for many GPCRs, NPFF2R activation by NPFF was found to recruit beta-arrestin 2 at the plasma membrane level of HEK cells (Elshourbagy et al., 
2000). Finally, it is worth mentioning that in differentiated SH-SY5Y cells, which express endogenous NPFF2 receptors, NPFF rapidly and transiently stimulated the ERK pathway, in a cAMP-dependent manner, and activated components of the NF- $\kappa \mathrm{B}$ system in an ERK-independent manner (Sun et al., 2012). NPFF was also found to stimulate the ERK pathway in SK-N-MC neuroblastoma cells (Anko \& Panula, 2006) while RFRPs inhibited gonadotropin gene transcription in gonadotrope L $\beta$ T2 cells via inhibition of AC/cAMP/PKA-mediated ERK activation (Son et al., 2012).

\subsection{PrRP-R signalling pathways}

Along with the discovery of the PrRP receptor, arachidonic acid metabolite release was first taken as a biological output for screening putative endogenous ligands on transfected CHO cells (Hinuma et al., 1998). Such a PrRP-mediated response was linked to the lipoxygenase pathway. Further examination of PrRP effects on primary or adenoma-derived pituitary cells (with endogenous PrRP-R expression) pointed to their ability to stimulate prolactin hormone (PRL) secretion (Hinuma et al., 1998).

There is now an overall consensus to consider functional $\mathrm{G}_{\mathrm{q}}$ coupling and $\mathrm{IP}_{3}$-induced calcium mobilization from intracellular stores as a major transduction pathway for PrRP receptors (Hinuma et al., 1998; Roland et al., 1999; Langmead et al., 2000). Both PrRP-20 and PrRP-31 behaved as full agonists and activated the receptor with equal potencies (Langmead et al., 2000; Engstrom et al., 2003; Elhabazi et al., 2013), suggesting a pivotal role for the amidated C-terminal part of these peptides (Hinuma et al., 1998; Roland et al., 1999; Engstrom et al., 2003). As shown in Table 3, PrRPs potencies in promoting a calcium response through PrRP-R activation well agreed with binding affinities.

PrRPs were first reported to have no relevant effects on either basal or forskolin-stimulated cAMP levels (Langmead et al., 2000). However, other studies pointed to the ability of these peptides to partially suppress cAMP production in pituitary cells (Hinuma et al., 1998) and of PTX to abolish by more than $80 \%$ the stimulation of $\left[{ }^{35} \mathrm{~S}\right]-\mathrm{GTP} \gamma \mathrm{S}$ binding to membranes of PrRP-R expressing CHO cells in response to PrRP-20 and -31 (Engstrom et al., 2003). The latter data thus suggested a possible coupling of PrRP-R to the $\mathrm{G}_{\mathrm{i} / \mathrm{o}}$ class of $\mathrm{G}$ proteins in these cells.

PrRPs (0.1-10 nM) were also found to significantly increase cAMP levels both in rat PC12 cells (Nanmoku et al., 2003; Samson \& Taylor, 2006) and in PrRP-R expressing CHO cells (Wang et al., 2012), supporting $\mathrm{G}_{\mathrm{s}}$ coupling. Addition of PrRPs to rat PC12 cells stimulated dopamine release as well as activity and expression of tyrosine hydroxylase, the enzyme responsible for the biosynthesis of catecholamines (Nanmoku et al., 2003, 2005). These effects were blocked by calcium chelators 
and reduced by PKA and PKC inhibitors, suggesting the involvement of $\mathrm{G}_{\mathrm{s}} / \mathrm{AC} / \mathrm{PKA}$ and $\mathrm{G}_{\mathrm{q}} / \mathrm{PLC} / \mathrm{PKC}$ pathways.

Possible regulation by PrRPs of the mitogen-activated protein kinase (MAPK) family has been investigated in rat RC-4B/C or GH3 pituitary tumor cells and in PC12 pheochromocytoma cells, which all express endogenous PrRP receptors (Hinuma et al., 1998; Takahashi et al., 2002; Nanmoku et al., 2003; Maixnerova et al., 2011). In RC-4B/C cells, PrRP-20 and -31 increased ERK1/2 and CREB phosphorylation and induced prolactin release (Maixnerova et al., 2011). In PC12 and GH3 cells, the activation of ERK and JNK by PrRPs was found to primarily involve $\mathrm{G}_{\mathrm{i} / \mathrm{o}}$ coupling, with a prominent role exerted by G $\beta \gamma$ heterodimers (Kimura et al., 2000; Nanmoku et al., 2005). p38 MAPK activity remained unaffected (Nanmoku et al., 2005). In GH3 cells, both ERK and JNK cascades were necessary to stimulate rat prolactin (rPRL) promoter activity, probably via the phosphorylation of an Ets transcription factor (Kimura et al., 2000). In PC12 cells, PrRP stimulation of ERK activity has been proposed to regulate tyrosine hydroxylase transcription, possibly through formation of an AP-1 complex (Nanmoku et al., 2005).

PrRP-R signalling also included Akt activation, a serine/theonine kinase known to play a central role in regulating key functions such as protein synthesis, glucose homeostasis, cell survival and death. In GH3 cells, PrRPs-induced Akt activation was mediated by $\mathrm{G}_{\mathrm{i} / \mathrm{o}}$ proteins, in a $\mathrm{G}_{\beta \gamma}$ dependent manner, and was partially dependent on PKC and extracellular calcium (Hayakawa et al., 2002). Blockade of Akt activation by wortmanin and examination of the effects of dominantnegative Akt or CREB proteins or of constitutively active Akt on rPRL promoter activity allowed the authors to suggest that PrRPs activation of a PI3Kinase/Akt cascade is necessary to induce rPRL promoter activity via a CREB-dependent mechanism. Recently, the PrRP receptor has been shown to play a key role in the pathogenesis of uterine fibroids, with PrRPs promoting specifically the proliferation of leiomyoma cells through activation of a PI3K-Akt-mTOR pathway (Varghese et al., 2013). Moreover, the aberrant expression of PrRP-R by leiomyoma cells was linked to the loss of transcriptional repression exerted by the REST tumor suppressor in the normal myometrum.

Further information on down-stream signalling pathways for the PrRP-R was provided by Lin and coworkers who identified a typical motif at the receptor C-terminus prone to elicit an interaction with PDZ domain proteins (Lin et al., 2001). Co-immunoprecipitation studies pointed the glutamate receptor interacting protein (GRIP), AMPA binding protein (ABP) and protein that interacts with C-kinase (PICK1) as potential interacting partners for both PrRP and AMPA receptors, suggesting that these receptors could be scaffolded together at the synapse level. More recently, PICK1 has been found to play a regulatory role in PrRP-R clustering and recycling (Madsen et al., 2012). 


\subsection{Kiss1R signalling pathways}

In various Kiss1R-transfected cell models, kisspeptins promote a robust calcium signal as the result of receptor coupling to the $\mathrm{G}_{\mathrm{q} / 11}$ protein, activation of phospholipase C (PLC), cleavage of phosphatidylinositol 4,5 biphosphate $\left(\mathrm{PIP}_{2}\right)$, and $\mathrm{IP}_{3}$-mediated calcium release from intracellular stores (Kotani et al., 2001a; Muir et al., 2001; Ohtaki et al., 2001). Kisspeptin-induced calcium mobilization was not affected by PTX (Kotani et al., 2001a) nor were basal or forskolin-elevated cAMP levels modulated by kisspeptins (Muir et al., 2001), supporting a lack of coupling of Kiss1R to $\mathrm{G}_{\mathrm{i} / \mathrm{o}}$ or $\mathrm{G}_{\mathrm{s}}$ proteins. Thus, and although kisspeptins may also trigger the release of arachidonic acid (Kotani et al., 2001a), monitoring of calcium mobilization became a popular functional assay to evaluate the agonist nature and potency of peptide derivatives at the Kiss1 receptor. As shown in Table 3, enly-kisspeptins were active in the low nanomolar concentration range while NPFF- and NPAF-related peptides, as well as RFRPs, PrRPs and QRFPs, did not bind to nor stimulated the Kiss1R. The N-terminally truncated peptides, kisspeptin-10 and -13 were three to ten times more active than kisspeptin-54 (metastin). Non-amidated metastin was weakly active (Ohtaki et al., 2001), again pointing to the importance of the C-terminally amidated sequence in receptor activation. To note an overall good correlation between peptide affinity and agonist potency.

On the other hand, the rise in intracellular calcium and diacylglycerol levels, as the result of enhanced $\mathrm{PIP}_{2}$ hydrolysis by Kiss1R-stimulated phospholipase $\mathrm{C}$, leads to protein kinase C (PKC) activation. In turn, activated protein kinase $\mathrm{C}$ initiates an array of intracellular phosphorylation events that appear to involve various subsets of kinases, depending on the cellular context. Indeed, in Kiss1R/CHO cells, kisspeptins induced a strong and sustained phosphorylation of ERK1/2 MAP kinases and a weak stimulation of p38 MAP kinase (Kotani et al., 2001a), similar to the pathway found in hypothalamic GnRH neurons (Liu et al., 2008). In a human placental cell line, kisspeptin10 transiently activated ERK1/2 and p38 MAP kinases and inhibited GSK3 beta activity through its phosphorylation (Roseweir et al., 2012). In anaplastic thyroid cancer cells with endogenous Kiss1R expression, metastin activated only ERK (Ringel et al., 2002) while it stimulated ERK and PI3K/Akt pathways in Kiss1R-null thyroid cancer cells stably transfected with Kiss1R (Stathatos et al., 2005). In a pancreatic beta cell line, kisspeptin-54 was reported to inhibit the expression of the transcriptional factor Isl-1 and insulin secretion via $\mathrm{Ca}^{2+} / \mathrm{PKC} / \mathrm{ERK} 1 / 2$ pathways (Chen et al., 2014). In a breast cancer cell line, the recruitment of GRK2 and $\beta$-arrestin- 2 in response to kisspeptin-10 was found a critical step for Kiss1R signalling towards ERK1/2 (Pampillo et al., 2009). In MEF fibroblasts, Kiss1R was reported to positively regulate ERK1/2 activity, using $\mathrm{G} \alpha_{\mathrm{q} / 11}$ and $\beta$-arrestin-2 pathways in a co-dependent and temporally overlapping manner. While $\beta$ arrestin-2 potentiated signalling to ERK, downstream of Kiss1R, $\beta$-arrestin-1 had the opposite 
effect (Szereszewski et al., 2010). The possibility for $\beta$-arrestins to mediate Kiss $1 \mathrm{R}$ signalling in their own right was further investigated in two recent studies. In mice lacking either $\beta$-arrestin-1 or -2, Kp-54-triggered GnRH secretion was significantly reduced as assessed from serum gonadotropin levels (Ahow et al., 2014). Conversely, following selective ablation of $\mathrm{G} \alpha_{\mathrm{q} / 11}$ in GnRH neurons, mice exhibited a milder reproductive phenotype as compared to wild-type but kept significant hormonal responses to Kp-54 administration (Babwah et al., 2015). These observations clearly indicated that Kiss1R remains signalling competent in the absence of $G \alpha_{\mathrm{q} / 11}$ and that it may

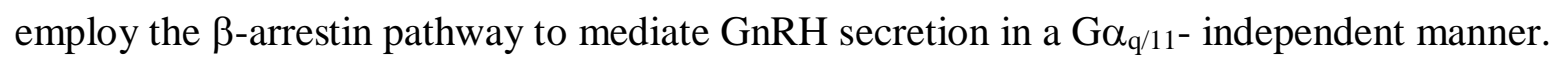

The physiological relevance of the Kiss1R/PLC/Ca ${ }^{2+}$ pathway was confirmed by pharmacological experiments performed on rat hypothalamic explants (Castellano et al., 2006a) as well as by electrophysiological recordings and calcium imaging studies in GnRH neurons from GnRH-GFP mice (Liu et al., 2008; Zhang et al., 2008; Dumalska et al., 2008). The G $\alpha_{\mathrm{q}}$ signalling pathway was clearly involved in the stimulatory effect of kisspeptins on GnRH secretion (Castellano et al., 2006a) and in the ability of these peptides to evoke a potent and sustained depolarising response in GnRH neurons (Dumalska et al., 2008, Liu et al., 2008, Pielecka-Fortuna et al., 2008, Zhang et al., 2008). Long-lasting depolarisation and increased firing rate of GnRH neurons mainly resulted from the concerted closure of inwardly rectifying $\mathrm{K}^{+}$(Kir) channels, opening of transient receptor potential (TRPC) channels, and inhibition of a slow afterhyperpolarization (sAHP) current. Kisspeptin-induced $\mathrm{PIP}_{2}$ hydrolysis inhibits Kir channels ( $\mathrm{K}_{\mathrm{ATP}}$ and GIRK channels) opening, a way to overcome the inhibition of GnRH neurons exerted by several $\mathrm{G}_{\mathrm{i} / \mathrm{o}}$ coupled receptors, such as Gaba в (Zhang et al., 2009) and $\mu$-opioid (Lagrange et al., 1995) receptors. In turn, activation of TRPC channels requires $\mathrm{PIP}_{2}$ depletion and cSrc kinase activation and is probably facilitated by calcium influx through low voltage-activated T-type calcium channels (Zhang et al., 2008; Zhang et al., 2013a; Ronnekleiv \& Kelly 2013). Kisspeptin inhibition of calcium-activated slow afterhyperpolarization (sAHP) currents was reported to involve protein kinase $\mathrm{C}$ activity and to facilitate a sustained firing of GnRH neurons (Zhang et al., 2013b). Once cell are depolarized, the opening of high voltage-activated $\mathrm{Ca}^{2+}$ (VGCCs) channels participates, together with TRPC channel activation and $\mathrm{Ca}^{+2}$ release from $\mathrm{IP}_{3}$ - and ryanodinesensitive stores, to the overall rise in intracellular $\mathrm{Ca}^{2+}$ concentration that leads to $\mathrm{GnRH}$ secretion (Zhang \& Spergel 2012; Ronnekleiv \& Kelly 2013).

Following the first description of KiSS-1 as a tumor suppressor gene (Lee et al., 1996), the effects of kisspeptins on cell mobility and chemotaxis, proliferation and cytoskeletal organization have been evaluated, together with underlying signalling processes. Among noticeable effects, metastin inhibited the chemotaxis of CHO cells towards fetal calf serum and of B16-BL6 melanoma cells towards fibronectin, only when Kiss1R was expressed in these cells (Ohtaki et al., 2001). 
Kisspeptin-10 inhibited trophoblast migration and their invasion of the uterine extracellular matrix, a process essential for fetal development (Bilban et al., 2004; Roseweir et al., 2012). Metastin induced also an excessive formation of focal adhesions and stress fibres in Kiss1R/B16-BL6 cells thereby explaining the observed reduction in cell motility- which was linked to the phosphorylation of focal adhesion kinase (FAK) and paxillin (Ohtaki et al., 2001). In Kiss1R/CHO cells, kisspeptin10 stimulated stress fibre formation, in a C3 exoenzyme-dependent manner, suggesting a possible intervention of the Rho subfamily of G proteins (Kotani et al., 2001a). Kisspeptins severely reduced the proliferation rate of various Kiss1R-expressing cell lines (Kotani et al., 2001a; Becker et al., 2005), at variance with expected effects of MAPK pathways in the control of cell cycle and proliferation.

A number of mechanisms have been postulated for kisspeptin/Kiss1R anti-metastatic and tumorsuppressor effects in numerous human cancers, including a role on matrix metalloproteinases and an interplay of Kiss1R with chemokine and EGFR receptors (Makri et al., 2008; Castano et al., 2009; Ji et al., 2013). It is important to mention here that, in breast cancer, elevated KiSS-1/Kiss1R expression was paradoxically correlated with worsened clinical outcome (Marot et al., 2007; Zajac et al., 2011; Cvetkovic et al., 2013).

\subsection{QRFP-R signalling pathways}

Pioneering work in the QRFP receptor field pointed to intracellular calcium mobilization as a major signal triggered by QRFP peptides in transfected CHO and HEK cells (Fukusumi et al., 2003; Jiang et al., 2003; Takayasu et al., 2006). These peptides also inhibited forskolin-induced cAMP accumulation in QRFP-R/CHO cells (Fukusumi et al., 2003). Thus, QRFP receptors may couple to both $\mathrm{G}_{\mathrm{q}}$ and $\mathrm{G}_{\mathrm{i} / \mathrm{o}}$ proteins. QRFP-26 has been reported to stimulate, though at high concentrations, cAMP production in rat pituitary cells pretreated with forskolin (Chartrel et al., 2003); the underlying mechanism remains to be clarified.

As shown in Table 3, QRFP-26 and QRFP-43 elicit a calcium response in the (sub) nanomolar concentration range, but they also inhibit cAMP production (Fukusumi et al., 2003). They are equipotent agonists in both assays, in agreement with their binding affinity for the human QRFP-R. Regardless the human, mouse or rat origin of QRFPs, the two mouse receptor isoforms were slightly less efficient than the human receptor in triggering intracellular calcium release, with $\mathrm{EC}_{50}$ values ranging from 73 to $194 \mathrm{nM}$ and from 42 to $70 \mathrm{nM}$, for QRFP-R1 and QRFP-R2 receptors respectively (Takayasu et al., 2006).

In the rat perfused pancreas, QRFP-26 was found to reduce the insulin secretion induced by either glucose, arginine or exendin-4, a GLP1 (glucagon-like peptide 1) analog that stimulates 
cAMP production. Such a response to QRFP-26 was suggested to involve $Q R F P-R / G_{i / o}$ coupling as it was absent in PTX-treated rats (Egido et al., 2007).

Activation of the QRFP receptor in human adrenocortical H295R cells also led to a decrease in cAMP production and to an increase of intracellular $\mathrm{Ca}^{2+}$ levels. QRFP treatment of these cells induced the secretion of aldosterone and cortisol which was found to involve PKC, MAPK and/or T-type $\mathrm{Ca}^{2+}$ channel-dependent pathways (Ramanjaneya et al., 2013). As discussed by the authors, future studies should delineate which particular G protein inhibitory $\alpha$-subunits couple to the QRFR receptor and how they may lead to an increase in $\left[\mathrm{Ca}^{2+}\right]_{\mathrm{i}}$ levels. Whether T-type calcium channels modulate QRFP-induced aldosterone secretion via an interaction with G $\beta \gamma$ subunits remains an interesting question. Indeed, their activity was found to be inhibited by certain $\beta \gamma$ dimers, independently of voltage, in a channel subtype-specific manner unique to the $\mathrm{Ca}_{\mathrm{v}} 3$ family (Wolfe et al., 2003).

\section{Structure-activity relationships studies on mammalian RF-amide peptides and non- peptidic ligands for $\mathrm{RF}$-amide receptors.}

Structure-activity/affinity relationship (SAR) studies are a key step towards a better comprehension of ligand features underlying high activity/affinity at their cognate receptors. They are also essential for the development of highly selective pharmacological tools (agonist or antagonist compounds) prone to unravel in vivo functional properties of given receptor subtypes under physio-pathological conditions. In the RF-amide field, several SAR studies have been conducted to define the minimal peptide sequence and the critical amino acids responsible for a high affinity and selective interaction with each RF-amide receptor. These studies, together with high-throughput screening campaigns, provided valuable information to develop several nonpeptidic compounds, exhibiting together greater stability, agonist or antagonist activities and therapeutic potential.

\subsection{SAR on the NPFF peptide scaffold}

Several modifications (amino acid deletions or substitutions) were introduced within the NPFF sequence (both at its $\mathrm{N}$ - or C-terminus) and the binding affinities of the resulting peptides were examined using $\left[{ }^{125} \mathrm{I}\right]\left[\mathrm{Tyr}^{1}\right] \mathrm{NPFF}$ (Gicquel et al., 1994) or $\left[{ }^{125} \mathrm{I}\right] 1 \mathrm{DMe}-\mathrm{NPFF}$ (Mazarguil et al., 2001) as the radioligands. Despite the lack of R1/R2 selectivity of these tracers (see Table 2), data presented in Table 4 most likely refer to the NPFF2 receptor as binding studies were performed on rat spinal cord preparations highly enriched in NPFF2R (Liu et al., 2001; Gouarderes et al., 2002, 
2004b). First of all, NPFF-OH (with a carboxylic acid group at its C-terminus) displayed no significant affinity, highlighting the common property of the RF-amide receptor family to bind Cterminally amidated neuropeptides. While deletion of the first two amino acids at the NPFF Nterminus did not alter their affinity towards NPFF receptors, NPFF(4-8) and NPFF(5-8) showed a reduction in affinity of 2 orders of magnitude. Further shortening of the NPFF sequence led to the tripeptide NPFF(3-8) with submicromolar affinity. Conservative substitution of $\mathrm{Phe}^{8}$ for another aromatic amino acid such as tyrosine ([Tyr $\left.{ }^{8}\right] \mathrm{NPFF}$ ), or of $\mathrm{Arg}^{7}$ for another basic residue ([Lys $\left.{ }^{8}\right]$ NPFF), both led to a marked decrease in affinity. The replacement of $\mathrm{Arg}^{7}$ or $\mathrm{Phe}^{8}$ residues with alanine, such as in $\left[\mathrm{Ala}^{7}\right] \mathrm{NPFF}$ and $\left[\mathrm{Ala}^{8}\right] \mathrm{NPFF}$, was much more deleterious. While substitution of the $\mathrm{Gln}^{6}$ residue for its acid counterpart ([Glu$]$ ]NPFF) also led to a significant reduction in affinity, modifications of the four first N-terminal NPFF amino acids were much better tolerated, highlighting the primary importance of the C-terminal RF-amide motif for high affinity binding to NPFF receptors.

Whether the typical Arg-Phe- $\mathrm{NH}_{2}$ signature also dictates functional activity for NPFF was further investigated by replacing $\mathrm{Arg}^{7}$ or $\mathrm{Phe}^{8}$ with a series of aliphatic and aromatic residues (Table 5). The potency and efficacy of NPFF analogs were determined from IP accumulation assays performed on COS-7 cells co-transfected with a chimeric G protein and human NPFF1 or NPFF2 receptors (Findeisen et al., 2011b). In agreement with former binding data (Table 4), substitution of $\mathrm{Phe}^{8}$ for Ala abolished NPFF agonist activity at NPFF1 and NPFF2 receptors. Interestingly, its replacement with non-aromatic cyclohexylalanine $\left(\left[\mathrm{Cha}^{8}\right] \mathrm{NPFF}\right)$ preserved significant activity at both receptors. Conversely, the substitution of Phe at the C-terminus by other aromatic amino acids such as Tyr, and especially Trp or His residues, led to a marked loss of peptide activity especially towards NPFF1R. Substitution of the natural L-Phe enantiomer for the D-Phe one resulted in a significant drop of agonist potency at both receptor subtypes. Elongation or shortening of the Phe side chain, such as in $\left[\mathrm{Hph}^{8}\right] \mathrm{NPFF}$ or $\left[\mathrm{Phg}^{8}\right] \mathrm{NPFF}$, severely impaired peptide potency at both receptors and impacted agonist efficacy especially at the NPFF1 receptor. Substitution of $\mathrm{Arg}^{7}$ by non-protonable side chains, such as in $\left[\mathrm{Ala}^{7}\right] \mathrm{NPFF}$ Ala or $\left[\mathrm{Cit}^{7}\right] \mathrm{NPFF}$, led to a drastic loss of activity, suggesting the requirement of a cationic group at this position. However, its replacement by charge-conserving residues (such as Lys or Orn) or by Arg analogs with shorter side chains (such as Agp and Apg), also markedly reduced peptide potencies at both receptors. Nevertheless, many of these compounds retained a full agonist activity toward NPFF2R, while exhibiting partial agonism at NPFF1 receptors.

Selectivity is also a critical point to evaluate the role of each individual receptor subtype. NPFF per se exhibits a modest selectivity towards NPFF2 receptor (Table 2). As the C-terminus part was considered as crucial for affinity and functional potency, Mazarguil and coworkers (Mazarguil et 
al., 2012) introduced an alanine-based sequence at the N-terminus of NPFF to obtain the $\mathrm{dA}(\mathrm{NMe}) \mathrm{AAFLFQPQRF}-\mathrm{NH}_{2}$ peptide that displayed a better NPFF2R selectivity $\left(\mathrm{K}_{\mathrm{i}}\right.$ values 0.16 $\mathrm{nM}$ and $12.1 \mathrm{nM}$ at NPFF2R and NPFF1R, respectively) essentially due to a decrease in affinity at the NPFF1R subtype.

\subsection{Dipeptidic and peptidomimetic agonists and antagonists of NPFF receptors}

A dipeptide derivative as small as dansyl-RF-amide was reported to suppress morphine-induced analgesia in vivo after peripheral administration, suggesting that the minimal Arg- $\mathrm{Phe}-\mathrm{NH}_{2}$ sequence was essential for anti-opioid activity (Brussaard et al., 1989). Indeed, this compound was later found to bind NPFF receptors in the rat spinal cord with fairly high affinity (Payza et al., 1993). Taking the Arg-Phe- $\mathrm{NH}_{2}$ motif as the minimal sequence required for peptide activity, Simonin and colleagues synthesized the dipeptide RF9 (Figure 1), with a bulky adamantoyl moiety at its $\mathrm{N}$-terminus, and provided evidence for its antagonist nature and similar binding affinity constants at human NPFF1 $(58 \mathrm{nM})$ and NPFF2 (75 nM) receptors (Simonin et al., 2006). More recently, independent studies reported on weak partial to full agonist activity of RF9 at NPFF1R and/or NPFF2R expressed in heterologous cell lines (Talmont et al., 2010; Findeisen et al., 2012; Kim et al., 2015). Overall, these findings raise the question of the relevance of the various in vitro assays built to study NPFFR functional properties. Such an important issue deserves further consideration. As RF9 interacted with other RF-amide receptors only in the low micromolar range, it was introduced as the first selective NPFF antagonist available (Simonin et al., 2006). In contrast with the former dansyl-RF- $\mathrm{NH}_{2}$ agonist, RF9 was able to prevent opioid-induced hyperalgesia and associated analgesic tolerance following its peripheral administration in rats (Simonin et al., 2006). RF9 was also found to display a strong gonadotropin-releasing activity in vivo in several mammalian species (Pineda et al., 2010a, b; Caraty et al., 2012a; Rizwan et al., 2012) that was suspected to involve an off-target action on kisspeptin receptors (Garcia-Galiano et al., 2012; Glanowska et al., 2014; Liu \& Herbison 2014; Sahin et al., 2015). Very recently, its Kiss1R agonistic component was clearly evidenced both in vitro and in vivo (Kim et al., 2015; Min et al., 2015), thereby questioning RF9 reliability as a pharmacological tool to delineate the role of NPFF receptors in the modulation of reproductive function.

An extensive structural exploration of the N-terminus of the Arg-Phe- $\mathrm{NH}_{2}$ sequence led to the identification of RF213, a novel dipeptide bearing a 2-phenylbenzoyl moiety (Gealageas et al., 2012). RF213 exhibited a subnanomolar affinity for NPFF1 receptors, similar to that of RFRP-3 $(\mathrm{NPVF})$, and an appreciable selectivity for NPFF1R $\left(\mathrm{K}_{\mathrm{i}}=0.3 \mathrm{nM}\right)$ versus NPFF2R $\left(\mathrm{K}_{\mathrm{i}}=590 \mathrm{nM}\right)$, and the ability to oppose fentanyl-induced hyperalgesia in rats. 
Both NPFF receptors exhibit a high structural homology with NPY receptors (Section 3.5.1.) and a significant affinity for the NPY Y1-selective BIBP3226 antagonist (Bonini et al., 2000; Mollereau et al., 2002). Inspired from previous works (Aiglstorfer et al., 2000; Mollereau et al., 2002), Kim and collaborators (Kim et al., 2015) reported on a NPFF antagonist structurally related to BIBP3226 (Figure 1). Based on a D-Arg-D-Phg backbone, this compound called GJ14 showed preferential binding affinity for the NPFF1 receptor subtype $\left(\mathrm{K}_{\mathrm{i}}\right.$ values $21 \mathrm{nM}$ and $387 \mathrm{nM}$ at NPFF1R and NPFF2R, respectively). Interestingly, GJ14 was found to exhibit anxiolytic properties, supporting NPFF receptors as novel potential targets for treating anxiety disorders. As a single but highly functionalized D-arginine residue, such as in BIBP3226 (Figure 1), may efficiently drive affinity and antagonistic properties at NPFF receptors (Mollereau et al., 2001, 2002; Kim et al., 2015), the C-terminus of the Arg-Phe- $\mathrm{NH}_{2}$ analog RF213 was deleted to afford the corresponding L-Arginine RF344 derivative (Figure 1). This compound was found to bind with high affinity ( $\mathrm{K}_{\mathrm{i}}$ close to 20 $\mathrm{nM}$ ) to both NPFF receptors (Bihel et al., 2015).

The significant affinity of the arginine BIBP3226, JG14 and RF344 derivatives for NPFF receptors highlight the requirement of a basic moiety (guanidine of arginine) combined with a hydrophobic group (phenyl ring). To go further with this simple pharmacophore pattern, the guanidine moiety was replaced with various secondary or tertiary amines, following a peptidomimetic approach. A new set of non-natural ornithine derivatives was developed and tested on both NPFF receptors (Bihel et al., 2015). The most interesting compounds (shown on Figure 1) were RF313 (NPFF1R: $K_{i}=172 \mathrm{nM}$; NPFF2R : $\mathrm{K}_{\mathrm{i}}=563 \mathrm{nM}$ ) and RF304 which exhibited a modest selectivity towards the NPFF2R subtype (NPFF1R: $K_{i}=400 \mathrm{nM}$; NPFF2R : $K_{i}=34 \mathrm{nM}$ ). As non-natural ornithine derivatives are much less polar $\left(\mathrm{RF} 313: \log \mathrm{D}=3.4, \mathrm{PSA}=63 \AA^{2}\right)$ than arginine derivatives, the probability they cross the blood-brain-barrier through a passive transport should be increased (Hitchcock, 2008). Indeed, RF313, despite its lower affinity for NPFF receptors when compared to RF9 or RF344, was able to prevent opioid-induced hyperalgesia in rats after oral administration (Bihel et al., 2015).

\subsection{Non-peptidic agonists and antagonists of NPFF receptors}

Dipeptides analogs were developed following a rational approach starting from the natural NPFF sequence. Alternatively, small non peptidic ligands of NPFF receptors were mainly identified from various chemical libraries following high throughput screening (HTS) campaigns. Benzoyl Gomisin Q and Gomisin G (Figure 2), two natural compounds isolated from the korean herb Schizandra chinensis, showed micromolar agonist potency at NPFF2R (Do et al., 2006). Interestingly, these compounds do not have any cationic moiety, suggesting that they bind to 
NPFF2R in a completely different way than arginine derivatives, although still keeping agonist activity.

Several pharmaceutical companies also discovered novel NPFF ligands (Figure 2). In 2003, Synaptic Pharmaceuticals patented quinolino- and quinazolino-guanidine derivatives (Forray et al., 2003; WO/2010/026657). The quinazoline series showed submicromolar and micromolar affinities for hNPFF1R and hNPFF2R, respectively, and were described as hNPFF1R antagonists and hNPFF2R partial agonists. The quinolone series bound with higher affinity to both NPFF receptors, showing a modest selectivity for the R1 subtype, and were described as antagonists. However, despite their drug-like character, quinazoline and quinazoline derivatives suffered from selectivity limitations, as they also displayed nanomolar affinities for a variety of receptors (NMDA, $\alpha_{2}$ adrenergic, NPY1) and transporters (serotonin and norepinephrine). In 2004, Actelion Pharmaceuticals patented a series of guanidinothiazole compounds (Caroff et al., 2004; WO/2004/083218) as hNPFF1R antagonists in the low nanomolar affinity range. However, no data were provided regarding the interaction of these compounds with the hNPFF2R and their antagonistic character at hNPFF1R was not documented. In 2009, Acadia Pharmaceuticals reported the discovery and the characterization of the first non-peptidic NPFF2R agonists in the micromolar range (Gaubert et al., 2009). Tested in vivo in rats, these aminoguanidines dose-dependently attenuated hypersensitivity in acute inflammatory and neuropathic pain models. Although structurally-related to Guanabenz, a well-known agonist of the $\square \alpha_{2}$-adrenergic receptor used to treat hypertension, these compounds did not show any agonistic activity at the $\square \alpha_{2 \mathrm{~A}}, \alpha_{2 \mathrm{~B}}$ or $\square \alpha_{2 \mathrm{C}}$ adrenergic receptors (Nguyen et al., 2014). Very recently, Journigan and collaborators described a novel series of ligands based on a generic guanidine-piperidine scaffold (Journigan et al., 2014). By simply modifying the functional groups linking the piperidine ring to the guanidine moiety and the aromatic platform, they were able to modulate the NPFF1/NPFF2 selectivity of ligands. While compound 53a (Figure 2) exhibited a modest preference towards the NPFF2R subtype (NPFF1R : $\mathrm{K}_{\mathrm{i}}=112 \mathrm{nM}$; NPFF2R : $\mathrm{K}_{\mathrm{i}}=30 \mathrm{nM}$ ), compound 46 was described as a NPFF1-selective antagonist $\left(\mathrm{NPFF} 1 \mathrm{R}: \mathrm{K}_{\mathrm{i}}=81 \mathrm{nM}\right.$; NPFF2R : $\left.\mathrm{K}_{\mathrm{i}}=1426 \mathrm{nM}\right)$. Both antagonists, at high concentration, only partially reversed the cAMP responses of NPFF1R or NPFF2R expressing cells to agonists. Noteworthy, compound $\mathbf{4 6}$ was able to prevent opioid-induced hyperalgesia (tail withdrawal test) following i.c.v. administration in mice, suggesting that NPFF1R is critically involved in this phenomenon.

\subsection{SAR on Prolactin-releasing peptides and their analogs}


The Prolactin-releasing peptides PrRP-20 and PrRP-31 (Table 1) were much less studied than NPFF or Kisspeptins. In 2005, Boyle and coworkers reported an extensive SAR of the N-truncated peptide (19-31)-PrRP (Table 6; Boyle et al., 2005). In agreement with the prominent role of the RFamide motif, PrRP with a C-terminal carboxylic acid was inactive. The systematic replacement of amino acids at different positions of (19-31)-PrRP pointed to a crucial role for the C-terminal heptapeptide. This SAR study confirmed previous work from Roland and collaborators who identified the heptapeptide (25-31)-PrRP as the shortest PrRP sequence preserving agonistic activity (Roland et al., 1999). However, a more recent study reported that $\mathrm{Phe}^{31}$ in PrRP-20 could be replaced with naphtylalanine or tyrosine, leading to compounds endowed with in vitro pharmacological properties comparable to those of PrRP-20 and able to elicit long-lasting anorexigenic effects after central administration in fasted mice (Maletinska et al., 2011). From a structural point of view, PrRP-20 exhibits an amphipathic $\alpha$-helical secondary structure with the residues $\mathrm{Ile}^{14}, \mathrm{Val}^{17}$ and $\mathrm{Phe}^{20}$ located on the same face of the helix (D'Ursi et al., 2002; DeLuca et al., 2013).

To date, no PrRP-R antagonists were described in peer-reviewed articles, but Otsuka Pharmaceuticals recently patented heterocyclic antagonists built on a tetrahydropyrido[4,3d]pyrimidinone scaffold (Takahashi et al., 2010; WO/2010/137738). These compounds display, like compound $\mathbf{2 3 8 6}$ (Figure 3), a high affinity for the PrRP receptor and were developed for the treatment of stress-related diseases.

\subsection{SAR on Kisspeptins and their analogs}

Since the discovery of the kisspeptin receptor (Kiss1R or GPR54) and of its endogenous ligands (Table 1), numerous structure-activity studies focused on the $\mathrm{N}$-terminally truncated sequences of kisspeptins (especially kisspeptin-10 and -13) and confirmed the C-terminus amide as a signature of this family of ligands (Table 7). Ala-scan experiments on kisspeptin-10 highlighted the critical role of ${ }^{6} \mathrm{Phe}$ and ${ }^{10} \mathrm{Phe}$ residues in terms of affinity and activity. Phe ${ }^{10}$ could be replaced by another aromatic residue (Trp, Tyr) without any loss of activity. $\mathrm{Arg}^{9}$ may be replaced by a basic amino acid such as lysine, but more surprisingly, its substitution for Ala preserved a potent agonist activity $\left(\mathrm{EC}_{50}=88 \mathrm{nM}\right)$ despite the loss of the electrostatic interaction of the guanidine residue. Finally, Phe $^{6}$ appeared to be one of the most important residues of the kisspeptin-10 sequence: this position did not tolerate many alterations and only aromatic residues preserved nanomolar binding affnity. NMR experiments on kisspeptin-13 in a membrane-like environment (SDS micelles) suggested a relatively stable helical conformation of the C-terminal part of the peptide (Orsini et al., 2007) while molecular modeling studies located the two important $\mathrm{Phe}^{6}$ and $\mathrm{Phe}^{10}$ residues on the same 
face of the helix (Gutierrez-Pascual et al., 2009). Taking into account these results highlighting the importance of the C-terminus sequence of kisspeptin-10, Niida and coworkers proposed a series of N-terminus acylated pentapeptides (FGLRWa) and identified the FM053a compound (Figure 4) as a full agonist (Niida et al., 2006; Tomita et al., 2006). A tryptophan residue at the C-terminus was preferred over phenylalanine as it slightly improved agonist activity at Kiss1R (Tomita et al., 2006). Further studies on this N-acylated pentapeptide series led to the agonist FTM080 (Figure 4), which was nearly as potent as kisspeptin-10 (Tomita et al., 2007, 2008a). However, FTM080 was found to be a substrate of matrix-metalloproteinase (MMPs) and to be cleaved between glycine and leucine residues in murine serum (Tomita et al., 2008b). To bypass such an enzymatic degradation, the authors inserted an appropriate dipeptide isoster into the pentapeptide sequence and found FTM145 (Figure 4 ) to be more stable (murine serum: $t_{1} / 2=38 \mathrm{~h}$ ) while keeping potent agonism at Kiss $1 \mathrm{R}$ (Tomita et al., 2008b). Other modifications of kisspeptin-10 were also found to enhance its in vivo stability. In 2010, Curtis and coworkers reported that the [dY]1KP-10 analog, with a ${ }^{\mathrm{T}} \mathrm{Tyr}$ at position 1, kept an in vitro bioactivity at Kiss1 R similar to that of kisspeptin-10 while displaying an improved efficacy to increase plasma LH and testosterone levels in mice (Curtis et al., 2010). $\mathrm{N}^{\mathrm{w}}$ methylation of $\mathrm{Arg}^{9}$, replacement of $\mathrm{Gly}^{7}$ with azaglycine, and substitution of $\operatorname{Trp}^{3}$ either by Ser, Thr, $\beta$-(3-pyridyl)alanine or by ${ }^{\mathrm{D}} \mathrm{Trp}$, led to a series of Kp-10 analogs that showed excellent Kiss1R agonist activity as well as higher stability in mouse serum (Asami et al., 2012a, b). All these modifications led to the disclosure by Takeda of compounds TAK-448 and TAK-683 (Matsui et al., 2012; Asami et al., 2013, 2014). Both pseudopeptides (Figure 4), when administered subcutaneously for several days to healthy men or prostate cancer patients, were well-tolerated and induced a sustained down regulation of testosterone into the castration range (MacLean et al., 2014; Scott et al., 2013). These two phase-1 studies indicated that the metastin analogs TAK-448 and TAK-683 may be promising candidates in clinical trials for the treatment of prostate cancer. Very recently, the triazololipopeptide 17 (Figure 4) that included a 1,2,3-triazole as a proteolysis-resistant amide bond mimic and an albumin-binding motif at its $\mathrm{N}$-terminus, was introduced as a highly potent kisspeptin analog able to trigger a prolonged increase of both LH and FSH plasma concentration in the ewe during the anoestrus season (Beltramo et al., 2015).

In 2009, the first Kiss1R antagonist, the peptide 234, was synthesized on the basis of the substitution in kisspeptin-10 of Leu $^{8}$ for D-Trp and of Ser ${ }^{5}$ for Gly (Roseweir et al., 2009). This peptide was able to reduce the pulsatile GnRH secretion in female pubertal monkeys and to inhibit the firing of GnRH neurons in murine brain. However, peptide 234 suffered from enzymatic degradation. In 2010, the Takeda pharmaceutical company isolated the first non-peptidic Kiss1R antagonists from a high-throughput screen of its internal chemical library. The antagonists 9I (IC 50 $=3.7 \mathrm{nM}$ ) and 15a $\left(\mathrm{IC}_{50}=3.6 \mathrm{nM}\right.$ ) belong to a new family of compounds (Figure 5) bearing a tetra- 
substituted pyridine scaffold (Kobayashi et al., 2010a, b; Doebelin et al., 2013). As a proof of concept, intravenous administration of 15a to castrated male rat suppressed plasma LH level, indicating the possibility for Kiss1R antagonists to act as a novel drugs for sex-hormone dependent diseases.

\subsection{SAR on QRFP peptides and their analogs}

Along with the discovery of QRFP-43 (or $43 \mathrm{RFa}$ ), Fukusumi and collaborators presented the first SAR study for QRFP neuropeptides (Fukusumi et al., 2003). QRFP-43 (with its typical Nterminal pyroglutamic acid) and the shorter QRFP-26 peptide (Table 1), which result from differential processing of the same precursor protein (see section 2.2.5.), display highest affinity and activity towards QRFP receptors (Table 3). As expected for RF-amide peptides, the carboxylic acid form of QRFPs was inactive. Moreover, serial deletion of the N-terminal sequence of QRFP-26 was found to gradually attenuate its affinity and potency (Fukusumi et al., 2003). The C-terminus decapeptide QRFP-(34-43) exhibited a submicromolar affinity for QRFP-R and very weak (if no) agonist activity; thus, QRFPs appeared to be much more sensitive to sequence shortening than other RF-amide peptides such as NPFF or kisspeptins. This observation is consistent with structural studies indicating a well-defined conformation of QRFP-26, with an amphipathic Ǔ-helical structure $\left(\right.$ Pro $^{4}-\mathrm{Arg}^{17}$ ) flanked by N- and C-terminal disordered regions (Thuau et al., 2005). Both the Cterminal sequence and the amphipathic helix seem important for QRFP-26 affinity and activity. However, a systematic Ala-scan and D-scan performed on the QRFP-(20-26) heptapeptide (GGFSFRF-NH $)_{2}$ showed that the last three C-terminal residues were very sensitive to substitution, while position 23 rather well tolerated both modifications (Le Marec et al., 2011). Most importantly, replacement of ${ }^{23} \mathrm{Ser}$ by a norvaline led to the agonist $\left[\mathrm{Nva}^{23}\right](20-26) 26 \mathrm{RFa}$ that was 3fold more potent $\left(\mathrm{EC}_{50}=233 \mathrm{nM}\right)$ than the native heptapeptide (Le Marec et al., 2011). Using a peptidomimetic approach applied to QRFP-(20-26), the same authors designed a series of aza- $\beta^{3}$ analogs and identified the $\left[\mathrm{Cmpi}^{23}\right.$, aza- $\left.\beta^{3}-\mathrm{Nva}^{23}\right](21-26) \mathrm{RFa}$ pseudopeptide as a stable and potent GPR103 agonist (LV-2172; Figure 6) with a long lasting orexigenic effect in mice (Neveu et al., 2012). To increase the metabolic stability of the QRFP-(20-26) heptapeptide, another peptidomimetic strategy targeted the N-terminal Gly-Gly pair which is prone to undergo enzymatic degradation (Pierry et al., 2013). The peptide bond was replaced by a fluorin-olefin moiety, which exhibits isosteric and isoelectronic properties similar to those of the native amide bond. The resulting $\left[\Psi^{20,21}(\mathrm{CF}=\mathrm{CH})\right](20-26) 26 \mathrm{RFa}$ compound (LV-2098; Figure 6) displayed a five-fold longer half-life (53 $\mathrm{min}$ as compared to $11 \mathrm{~min}$ for the heptapeptide) in human serum and an activity similar to that of the parent QRFP-(20-26) peptide (Pierry et al., 2013). 
Only a few QRFP-R antagonists were disclosed (Figure 6). In 2010, Banyu Pharmaceuticals patented a series of indole derivatives (Haga et al., 2010; WO/2010/126164). More recently, AstraZeneca developed non peptidic antagonists based on a pyrrolo[2,3-c]pyridine scaffold. The lead compound (25e ; Figure 6) has an $\mathrm{IC}_{50}$ of $40 \mathrm{nM}$ and better drug metabolism, pharmacokinetics and safety properties than the former indole derivatives (Georgsson et al., 2014). Using NMR in combination with conformational studies, these authors claimed that the peptidic (20-26)RFa agonist and the pyrrolo[2,3-c]pyridine could share overlapping pharmacophores, the antagonist mimicking the C-terminal $\mathrm{Arg}^{25}-\mathrm{Phe}^{26}$ residues of the endogenous peptide (Georgsson et al., 2014). At the same time, AstraZeneca published the results of a high throughput screening performed on 900,000 compounds which led to the identification of three chemical families, and in particular of the compound 2 (Figure 6) with an $\mathrm{IC}_{50}$ of $80 \mathrm{nM}$ in a binding assay (Nordqvist et al., 2014).

In summary, most SAR studies in the RF-amide field have been conducted starting from endogenous peptides. Although novel compounds exhibited interesting pharmacological properties, most of them are of peptidic nature and thus display a limited ádruggabilityô Many of these compounds have been optimized for their affinity/potency towards a given receptor but their selectivity profile for the entire RF-amide receptor family has rarely been reported. This is a critical issue if one remembers the propensity of NPFF receptors to bind most endogenous RF-amide peptides. Off-target actions and possible intervention of other receptors involved in the biological question of interest should also be considered when using novel pharmacological tools and interpreting functional data. Only a few non peptidic compounds were described, probably owing to the difficulty to develop heterocyclic compounds from peptidic ligands. However, using rational design or high throughput screening, some heterocyclic structures are emerging, but none of them reached clinical studies yet.

\section{Mammalian RF-amides peptides and their receptors in feeding and metabolism}

\subsection{Overview on energy homeostasis and regulation of food intake}

Feeding behaviour is intimately linked to energy homeostasis, a finely tuned equilibrium between energy intake (food consumption), energy expenditure (metabolism, thermogenesis, muscle activity) and energy storage (adipogenesis). Many endocrine and metabolic signals, in combination with genetic, cognitive, emotional and environmental factors, provide nutritionallyrelevant information to a central homeostatic regulator that generates, via efferent neural circuits, appropriate responses to meet caloric needs and maintain energy balance. 
Peripheral signals (neuropeptides and nutrients) released from the gastrointestinal tract, such as cholecystokinin (CCK) and ghrelin, from the pancreas (insulin, glucagon) and the adipose tissue (leptin, adiponectin) target specialized hypothalamic nuclei, either directly through the blood circulation and/or indirectly, via vagal and sympathetic input to relaying brain stem nuclei.

The hypothalamic network includes several interconnected nuclei, such as the arcuate (ARC), paraventricular (PVN), ventromedial (VMH), dorsomedial (DMH) and supraoptic (SON) nuclei and the lateral hypothalamic (LHA) and preoptic (POA) areas, that integrate peripheral and central information and exert, via a variety of orexigenic (such as NPY, Agouti-related peptide, galanin or orexin) and anorexigenic (such as proopio-melanocortin (POMC), melanocyte-stimulating hormone $(\alpha-\mathrm{MSH})$ or oxytocin) neuropeptides and neurotransmitters, a bi-directional control of food intake and metabolism. As a nodal homeostatic centre, the hypothalamus communicates with the dorsovagal complex (nucleus of the solitary tract (NTS), area postrema (AP), dorsal motor nucleus of the vagus $(\mathrm{DMX})$ ) in the brainstem and the lateral parabrachial nucleus (PB) in the midbrain to regulate consummatory aspects of feeding (including taste aversion and satiety), with the cortico-limbic system (ventral tegmental area (VTA), nucleus accumbens (NAc), amygdala (Amd), orbitofrontal cortex (OFC)) to integrate the reward and hedonic valuation of food, and with the hypothalamicpituitary-gonadal (HPG) axis to coordinate appetite and energy metabolism with reproductive state. It also responds to circadian influences and interacts with neurons involved in thermoregulation.

Deeper insights and expert comments have been published in several recent reviews devoted to the homeostatic control of food intake and energy metabolism (Lenard \& Berthoud, 2008; Parker \& Bloom, 2012; Williams \& Elmquist, 2012; Yeo \& Heisler, 2012; Schwartz \& Zeltser, 2013; Morton et al., 2014), to the dysregulation of reward systems in eating disorders (Volkow et al., 2011; Avena \& Bocarsly, 2012; Meye \& Adan, 2014; Singh, 2014) and to the link between nutrition and reproduction (Evans \& Anderson, 2012; Hirschberg, 2012; Navarro \& Tena-Sempere, 2012; Schneider et al., 2013).

More specifically, current knowledge on the involvement of the RF-amide system in feeding behaviour and energy balance will be presented hereafter.

\subsection{RFRPs and the NPFF1 receptor}

7.2.1. Distribution of RFRPs and their receptor in peripheral organs and CNS areas involved in energy homeostasis.

The localization of RFRP-containing neuronal cell bodies and of NPFF1 receptors, in relation with a possible implication in the control of food intake, is presented on schematic sagittal sections of rodent brain (Figure 7). Further details on the localization of RFRP peptides and of NPFF1R in 
Mammals are available in dedicated reviews (Fukusumi et al., 2006; Smith \& Clarke, 2010; Parhar et al., 2012).

Localization of RFRP neuronal cell bodies : As assessed from in situ hybridization (ISH) and immunohistochemistry (IHC) studies performed on mouse (Ukena \& Tsutsui, 2001; Kriegsfeld et al., 2006), rat (Hinuma et al., 2000; Fukusumi et al., 2001; Yano et al., 2003; Kriegsfeld et al., 2006; Johnson et al., 2007; Legagneux et al., 2009; Rizwan et al., 2009), hamster (Kriegsfeld et al., 2006), ovine (Clarke et al., 2008; Dardente et al., 2008; Smith et al., 2008a) and macaque (Ubuka et al., 2009a) brain, RFRP-positive neuronal cell bodies locate within the hypothalamus, essentially in the paraventricular (PVN) and dorsomedial (DMH) nuclei and between the DMH and ventromedial (VMH) nuclei.

Localization of RFRP fibers : No significant levels of immunoreactive (ir) RFRPs nor RFRPs mRNA were detected in peripheral tissues. In contrast, RFRP-ir neuron projections and nerve terminals have been visualized in a range of brain areas in rat, mouse, hamster, ovine and primate (Ukena \& Tsutsui, 2001; Yano et al., 2003; Gibson et al., 2008; Qi et al., 2009; Ubuka et al., 2009a). They include major structures involved in energy balance, such as the arcuate (ARC), dorsomedial (DMH) and paraventricular (PVN) nuclei as well as the preoptic (POA) and lateral (LHA) areas of the hypothalamus, the amygdala (Amd) in rat, mouse and hamster, and more caudal areas such as the lateral parabrachial nucleus (PB) and the nucleus of the solitary tract (NTS). In the ovine brain, retrograde tracer labelling further demonstrated that RFRP-3 neurons in the PVN and DMH nuclei project onto the ARC, LHA, VMH and POA (Qi et al., 2009) where the NPFF1 receptor is expressed.

Double-label immunohistochemistry in ovine brain allowed to identify a subset of RFRP-3 neurons in close apposition with NPY- or POMC- producing cells in the ARC and with orexin or melanin-concentrating hormone $(\mathrm{MCH})$ neurons in the LHA (Qi et al., 2009). Moreover, RFRP neuronal cell bodies in the DMH were found to project onto GnRH neurons in the preoptic area of human (Ubuka et al., 2009b), hamster (Kriegsfeld et al., 2006), sheep (Smith et al., 2008a) and monkey (Ubuka et al., 2009a) brain, suggesting that RFRPs are involved in the regulation of GnRH neurons.

Distribution of NPFF1R (GPR147) in mammalian tissues : As assessed from RT-qPCR analyses, NPFF1 receptors are expressed in the rat testis (and to a much lower extent in the ovary, placenta and retina) and show a broad distribution in the human and rat CNS (Bonini et al., 2000; Hinuma et al., 2000). NPFF1R localization in rat brain and human hypothalamus was also examined using ISH (Liu et al., 2001) and IHC (Goncharuk et al., 2004) approaches. More quantitative insights on the anatomical distribution of the NPFF1 receptor in the CNS of various mammalian species were obtained from autoradiography, a technique aiming at localizing specific radioligand binding sites 
on brain tissue sections. First studies were performed on rat brain, using [ $\left.{ }^{125} \mathrm{I}\right]-\mathrm{Y} 8 \mathrm{Fa}$ (Allard et al., 1992) and [ $\left.{ }^{125} \mathrm{I}\right]-1 \mathrm{DMe}$ (Dupouy et al., 1996; Dupouy \& Zajac, 1996), two tracers that do not discriminate among NPFF receptors subtypes (Table 2 and Section 4.1.1). To increase labelling specificity, subsequent autoradiographic studies included the frog pancreatic peptide (fPP) to abolish an undesirable NPFF2 binding component (Bonini et al., 2000) and took advantage of the more NPFF1-selective [ $\left.{ }^{125} \mathrm{I}\right]-Y V P$ radioligand to investigate NPFF1 receptor distribution (Gouarderes et al., 2002; Gouarderes et al., 2004a,b). Collectively, and within the context of feeding regulation and energy homeostasis, all these data pointed to the presence of the NPFF1R in the ARC and PVN hypothalamic nuclei, its low to moderate expression in other hypothalamic regions (DMH and VMH nuclei, LHA and POA areas), in the amygdala (Amd), and in brainstem (AP, NTS) regions (Liu et al., 2001; Gouarderes et al., 2002; Goncharuk et al., 2004; Gouarderes et al., 2004b).

It is worthwhile mentioning the existence of discrepancies in NPFF1R density profiles between ISH, IHC and autoradiography studies, but also between the different mammalian species studied so far (Goncharuk et al., 2004; Gouarderes et al., 2004b) and even between mouse or rat strains (Gouarderes et al., 2004a). For example, in Sprague-Dawley rats, [ $\left.{ }^{125} \mathrm{I}\right]-Y V P$ autoradiography showed the presence of the NPFF1R in the nucleus accumbens (NAc) and its absence in the ventral tegmental area (VTA), with the reverse observation being true in Wistar rats (Gouarderes et al., 2004a). Conversely, immunoblotting on extracts from the same regions of Sprague-Dawley rats indicated abundant NPFF1R in the VTA and a few in the NAc (Wu et al., 2010). This emphasizes the importance of comparing receptor (and peptide) distribution patterns afforded from various techniques and the great caution that should be taken when analyzing IHC data that used antibodies that have not yet been fully validated. Nevertheless, the possibility for NPFF receptors to localize on dopaminergic (and/or GABAergic) neurons in the rat VTA should not be excluded as double immunohistochemical staining suggested the expression of NPFF1R and NPFF2R on tyrosine hydroxylase- (or glutamic acid decarboxylase-) positive neurons (Wu et al., 2010).

\subsubsection{Effect of RFRPs on food intake}

Despite the considerable interest RFRP peptides and their NPFF1 (GPR147) receptor received as members of an opponent system to kisspeptin/Kiss1R in reproduction (Section 8) and the prominent link between the reproductive and metabolic status, less studies addressed their contribution in the control of food intake and energy balance in Mammals.

Similarly to effects of GnIH originally reported in birds (Tachibana et al., 2005), RFRP-3 (i.c.v) was found to increase food intake within 2 hours during photophase in male rats fed ad libitum (Johnson et al., 2007; Johnson \& Fraley, 2008; Murakami et al., 2008) as well as in male mice, 
ewes and non-human primates (Clarke et al., 2012). The i.c.v. administration of RFRP-3 to rats also caused a rapid and strong expression of the c-Fos immediate early gene (taken as a marker of neuronal activity) in several brain areas involved in the regulation of food intake and energy balance, such as the ARC, SON and rostral portion of the NTS (Yano et al., 2003). In sheep, RFRP3 infusion increased c-Fos labelling in orexigenic (NPY) as well as in anorexigenic (POMC) cells of the arcuate nucleus (Clarke et al., 2012). This paradoxical activation of POMC cells by RFRP-3 remains to be clarified since other studies reported a negative effect of this peptide on the firing of POMC neurons in mouse brain (Fu \& van den Pol 2010).

In marked contrast with former findings, anorectic effects of RFRF-1 (Kovacs et al., 2012) and RFRP-3 peptides (Kovacs et al., 2014) have been reported in rats on a liquid diet, following microinjection of the peptides into the amygdala. These effects were suppressed by the NPFF1/2 receptor antagonist RF9. The reasons for such striking discrepancies regarding the effects of RFRPs on food intake remain unclear but a possible link between an RFRP-3-elicited anxiogenic behaviour in rats (Kaewwongse et al., 2011) and the hypophagic effect of this peptide deserves further investigation.

\subsubsection{Effect of RFRPs on energy homeostasis}

I.c.v. injection of RFRP-3 in mice induced a significant hypothermia which was fully prevented by co-administration of the R1/R2 selective NPFF antagonist RF9 (Quelven et al., 2005; Mouledous et al., 2010a). RFRP-mediated decrease in body temperature was consistent with the presence of NPFF1R in several hypothalamic regions involved in thermoregulation in rodents (Ishiwata et al., 2001; Romanovsky, 2007). A more recent study pointed to a lack of effect of RFRP-3 on overall energy expenditure in male rats and on thermogenesis in female sheep that contrasted with the

general rule for áppetite-regulatingôpeptides that predicts opposite effects on food intake and energy expenditure (Clarke et al., 2012). Interestingly, the authors suggested that RFRP-3 may function as a molecular switch between feeding and reproductive behaviour, with high RFRP-3 activity favouring feeding over reproduction.

\subsubsection{Effect of feeding status on RFRPs/NPFF1R expression}

The number of RFRP-immunoreactive neuronal cell bodies in the DMH of Syrian hamster females was found to decrease following food restriction while their activity increased, as assessed from c-Fos labelling (Klingerman et al., 2011). Food intake remained similar to that of animals fed ad libitum but food hoarding significantly increased.

The impact of extreme metabolic changes was examined in wild-type and NPFF1 receptor null mice (Leon et al., 2014). Although both wild-type and NPFFR1 KO animals displayed a similar body weight in adulthood, complete food deprivation was found to accelerate weight loss in $\mathrm{KO}$ 
mice, in agreement with the expected function of RFRPs as orexigenic factors. High-fat diet evoked a similar increase in body weight in both strains but one cannot exclude that such modest alterations might be due to compensatory mechanisms.

Food availability and photoperiod have been shown to differentially impact RFRPs (in the DMH) and KiSS-1 (in the ARC) genes expression in male Syberian hamsters (Paul et al., 2009). Shorter photoperiods increased RFRP mRNA levels - although this point has been disputed (Revel et al., 2008) - while they remained unaffected by food restriction. At the opposite, KiSS-1 mRNA markedly decreased in animals exposed to reduced food availability whereas they were insensitive to the photoperiod. Such responses, which are unlikely to be driven by altered gonadal steroid production, suggest a specific recruitment of these RF-amide systems in the integration of photic and metabolic cues for the control of reproduction.

Light-induced neuronal activity of the suprachiasmatic nucleus (SCN) signals rest in nocturnal rodents and activity in diurnal primates (Houben et al., 2009). Such a circadian control is overridden when food access is restricted to a predictable episode during the rest phase, so that animals become active and anticipate the feeding event. Food anticipatory activity (FAA) has been recently found to be modulated by the interaction of $\mathrm{DMH}$ and $\mathrm{SCN}$ nuclei, within a hypothalamic network comprising RFRP fibers (Acosta-Galvan et al., 2011). Interestingly, this study demonstrated the activation of some RFRP neurons in the DMH during FAA and their projection onto vasoactiveintestinal and gastrin-releasing peptides neurons in the SCN, in close association with afferent fibers arriving from the retina.

\subsection{NPFF/NPAF and the NPFF2 receptor}

\subsubsection{Distribution of NPFF and NPAF and of their receptor in the CNS and in peripheral organs} involved in energy homeostasis

The localization of NPFF/NPAF-containing cell bodies and of NPFF2R in rodent brain, in relation with their known or putative roles in the control of food intake, is presented in Figure 7. Localization of NPFF/NPAF-containing neuronal cell bodies : The distribution of NPFF, NPAF and of their proNPFF A precursor is essentially from ISH and IHC studies performed on rat brain.

Highest levels of NPFF-containing neurons were found in the nucleus of the solitary tract (NTS), significant expression also occurred in the supraoptic (SON) and paraventricular (PVN) hypothalamic nuclei, while the existence of NPFF-ir neuronal cell bodies in the dorsomedial $(\mathrm{DMH})$, ventromedial (VMH) and arcuate (ARC) nuclei was more ambiguous (Kivipelto et al., 1989; Boersma et al., 1993; Lee et al., 1993; Panula et al., 1996; Vilim et al., 1999; Goncharuk et al., 2006). A comparative semi-quantitative immunohistochemical analysis of the distribution of 
NPFF in rat and human forebrain revealed a similar pattern in both species, with a noticeable difference in the human SON which exhibited very low NPFF-ir levels (Goncharuk et al., 2006).

Localization of NPFF/NPAF-containing neuronal fibers and terminals: Immunoreactive NPFF fibers and nerve terminals exhibit a wide distribution in mammalian brain, including the hypothalamus (POA, ARC, DMH, PVN and VMH nuclei), limbic areas (NAc, amygdala), and the lateral parabrachial nucleus (PB) in the midbrain (Kivipelto et al., 1989; Panula et al., 1996; Vilim et al., 1999; Goncharuk et al., 2006).

Localization of NPFF2R : The distribution of NPFF2R in mammalian tissues has been examined using RT-qPCR, ISH, IHC and autoradiography, often in parallel with NPFF1R.

As shown from RT-qPCR performed on peripheral tissues, significant NPFF2R mRNA levels were detected in the rat (Bonini et al., 2000) and human (Elshourbagy et al., 2000) adipose tissue. Low to moderate NPFF2R expression was also reported in rat retina, salivary gland, stomach, intestine and liver (Bonini et al., 2000).

Mapping of NPFF2R mRNA in rat brain areas showed a limited pattern of expression, including the medulla (NTS, dorsal motor nucleus of the vagus), the hypothalamus (LHA) and the amygdala (Bonini et al., 2000; Liu et al., 2001). Immunohistochemical detection of NPFF2 receptors in the human brain provided a more precise profile : in the forebrain, highest receptor density was found in the amygdala and DMH and moderate to low expression in the VMH, LHA, PVN and VTA; within the medulla oblongata, the dorsal motor nucleus of the vagus nerve (DMX) and the region of catecholaminergic cell groups $(\mathrm{A} 1 / \mathrm{C} 1)$ were the most enriched areas in NPFF2R (Goncharuk \& Jhamandas, 2008).

Autoradiographic studies using the NPFF2R selective $\left[{ }^{125} \mathrm{I}\right]-\mathrm{EYF}$ tracer under controlled conditions (Gouarderes et al., 2001, 2002, 2004b), indicated a wider distribution and a considerably greater density of NPFF2R in the rat brain, as compared to NPFF1R. Brainstem (NTS and PB) and hypothalamic (VMH, LHA, POA and ARC) nuclei as well as areas belonging to the dopaminergic mesolimbic system (VTA, NAc, caudate putamen) exhibited significant levels in NPFF2 binding sites. To note that some NPFFergic neurons from the NTS project within the lateral parabrachial nucleus (Kivipelto \& Panula, 1991; Panula et al., 1996; Jhamandas et al., 2001) where mu-opioid receptors are expressed (Chamberlin et al., 1999; Nicklous \& Simansky, 2003).

Autoradiographic studies also pointed to marked differences in the distribution of NPFF2 receptor binding sites according to species (Dupouy \& Zajac, 1996; Gouarderes et al., 2004b) and rodent strain (Gouarderes et al., 2004a). No NPFF2R sites were detectable in the central nervous system of the diurnal Octodon Degus rodent nor in the olfactory bulb (OB) of rats, in marked contrast with mice that exhibited a high density of binding sites in this latter region. As the olfactory bulb is known to contribute to the evaluation of hedonic properties of food and to contain a circadian clock 
that participates to feeding anticipation (Nolasco et al., 2012; Caba et al., 2014), a direct participation of NPFF2R in olfactory bulb-linked feeding behaviour in rats is unlikely.

Although available data revealed some species- and methodological-dependent variations in the relative density of endogenous proNPFF A peptides or NPFF2R within brain areas, they provided an overall distribution pattern that is consistent with their role in feeding regulation.

\subsubsection{Effect of NPFF and NPAF on food intake}

As an introductory remark, it is important to keep in mind that proNPFF${ }_{A}$-derived peptides poorly discriminate between NPFF1 and NPFF2 receptors in terms of binding affinity. Although such a drawback might be compensated for by a greater functional selectivity (Table 3), high local peptide concentrations may be reached when injected into the brain. Therefore, data need to be interpreted with much caution unless highly selective NPFF1 or NPFF2 receptor antagonists or genetic invalidated mice are used.

NPFF is involved in the modulation of diverse feeding-related processes, including intestinal motility (Decker et al., 1997; Fang et al., 2005), osmoregulation (Majane \& Yang, 1991; Sunter et al., 2001; Kalliomaki \& Panula, 2004) and food intake (Murase et al., 1996; Sunter et al., 2001; Nicklous \& Simansky, 2003; Dockray, 2004).

Initial studies reported that i.c.v. injections of NPFF induce a rapid, transient and dose-dependent reduction in food intake in fasted rats (Murase et al., 1996; Sunter et al., 2001) and in ad libitum fed mice (Bechtold \& Luckman, 2006). In rats, the hypophagic action of NPFF was first attributed to the concomitant large increase in water intake induced by the peptide (Sunter et al., 2001) but no significant dipsogenic effects of NPFF (Nicklous \& Simansky, 2003) or NPAF (Newmyer \& Cline, 2011) were detected in subsequent studies. NPFF-induced hypophagia is unlikely to result from an interference with satiety signalling sequences as the activity of NPFF neurons within the NTS was insensitive to the satiety factor CCK (Bechtold \& Luckman, 2007). In turn, i.c.v. administration of NPFF was found to activate oxytocin neurons in the PVN (Jhamandas \& MacTavish, 2003), suggesting the participation of the anorectic oxytocin peptide in NPFF-induced hypophagia.

The well-known anti-opioid effects of NPFF in nociception (Section 9) and orexigenic action of opioid agonists (Morley et al., 1983; Yim \& Lowy, 1984), opposite to that of NPFF, raised the possibility for an interplay between both systems in the regulation of food intake. Indeed, the central administration of FMRF-amide peptides in mice or of NPFF in rats dose-dependently prevented hyperphagia induced by mu or kappa opioid receptor agonists (Kavaliers \& Hirst, 1986; Nicklous \& Simansky, 2003). At higher doses, NPFF infusion into the lateral parabrachial nucleus markedly increased food intake in freely feeding rats, with a delayed onset and a prevention by naloxone very similar to mu-opioid responses (Nicklous \& Simansky, 2003). Thus, NPFF may 
exert pro- and anti-opioid effects on food intake, very similarly to its capacity to induce antinociception or to oppose opioid-induced analgesia (Section 9.2.2).

\subsubsection{Effects of NPFF, NPAF and NPSF on energy homeostasis}

EFW-NPAF or dNPA (two NPFF2R preferring peptides) reversed the hypothermia evoked by saline injection in mice when injected into the third ventricle (Quelven et al., 2005; Mouledous et al., 2010a). This response, which was blocked by the NPFF antagonist RF9, is probably due to NPFF2R-induced hyperthermia. However, given the bell-shape dose-response curves for these agonists and the decrease in body temperature consecutive to the administration of the poorly NPFF2R-selective NPFF and 1DMe peptides in mice (Desprat \& Zajac, 1997; Zajac et al., 2000; Quelven et al., 2005; Fang et al., 2008), one cannot exclude that NPFF2R-driven hyperthermia might be dominated by the hypothermic NPFF1R response in case of co-stimulation of both NPFF receptors (Mouledous et al., 2010a).

NPFF and NPAF peptides were found to modulate the expression of a number of genes involved in adipose metabolism in 3T3-L1-differentiated rodent fat cells (Lefrere et al., 2002). Up-regulation of beta 2 and beta3-adrenergic receptor genes was accompanied with an increase in receptor expression and potency of beta adrenergic agonists to stimulate adenylate cyclase activity in NPAFand NPFF-treated cells. Thus, these RF-amide peptides may impact body energy storage and utilization via activation of beta-adrenergic receptors known to activate lipolysis and thermogenesis and to exert a negative control on lipogenesis and glucose transport (Lafontan \& Berlan, 1993). To note however that NPFF, NPAF and NPSF failed to modify lipid accumulation in 3T3-L1 cells while they partially reduced adipogenesis in 3T3-F442A preadipose fat cells (Herrera-Herrera \& Salazar-Olivo, 2008). As peptide potencies did not correlate with their affinities for NPFF2 receptors, the occurrence of alternative signalling pathways was suggested.

In marked contrast with former data obtained on rodent adipocytes, NPFF was found to inhibit catecholamine-induced lipolysis in human fat cells, via NPFF2R activation (Dahlman et al., 2007). Similar responses to NPFF and NPSF were observed in human adipocytes differentiated in vitro from adipocyte precursor cells, taken either from non-obese or obese donors, while the expression of key genes involved in lipolysis modulation (including beta-adrenergic receptor genes) remained unaffected (van Harmelen et al., 2010). Altogether, these data indicate that NPFF, NPAF and NPSF peptides may display marked species differences in their ability to regulate lipolysis and fat storage.

\subsubsection{Metabolic context and genetic predisposition to obesity}

Hyperosmolar stress of rats was reported to down-regulate NPFF gene transcription in the SON and PVN nuclei (two hypothalamic nuclei involved in body fluid homeostasis), independently from 
the vasopressin regulatory system, but underlying mechanisms remain to be clarified (Kalliomaki \& Panula, 2004).

Chronic food restriction or diet-induced obesity in rats did not affect NPFF2R mRNA levels in the hypothalamus (Laemmle et al., 2003). Conversely, a clinical examination of the relative expression of NPFF1 and NPFF2 receptors in the adipose tissue from non obese and obese individuals showed a significantly lower NPFF2R mRNA level in the obese cohort (van Harmelen et al., 2010).

Among candidate marker genes of obesity, MC4R (melanocortin-4 receptor) and FTO (fat mass and obesity associated protein) genes show frequent prevalence of mutations or polymorphisms related to obesity (Dina et al., 2007; Loos et al., 2008). Looking at single-nucleotide polymorphisms in the NPFF2R gene, some haplotypes have been associated with an increased risk of obesity whereas others were strongly protective against obesity, in association with higher lipolytic activity (Dahlman et al., 2007; Hunt et al., 2011). The authors concluded that NPFF2R may play an important role in obesity predisposition, independently of NPY2R, FTO and MC4R loci.

\subsection{PrRPs and the PRRP receptor}

\subsubsection{Distribution of PrRPs and their receptor in peripheral organs and CNS areas involved in} energy homeostasis.

The brain distribution of PrRP-containing cell bodies and of the PrRP receptor, in light with their known or putative roles in feeding behaviour in Mammals, is presented in Figure 7. Detailed localization of PrRPs and their receptor in peripheral and central tissues has been published in several reviews (Sun et al., 2005; Fukusumi et al., 2006; Lin, 2008; Dodd \& Luckman, 2013).

Localization of PrRP-containing neurons and cell bodies : PrRP mRNA was detected in a limited set of peripheral organs involved in feeding such as the pancreas, the stomach and possibly the intestine (Fujii et al., 1999; Roland et al., 1999; Nieminen et al., 2000). As assessed from RT-PCR, ISH and IHC studies performed in rat brain, PrRP cell bodies concentrate within the nucleus tractus solitarius (NTS), and to a lesser extent in the ventrolateral medulla (VLM) of the caudal brainstem and the dorsomedial hypothalamic (DMH) nucleus (Fujii et al., 1999; Iijima et al., 1999; Maruyama et al., 1999; Ibata et al., 2000; Lee et al., 2000). In the VLM and NTS, PrRP co-localizes with tyrosine hydroxylase within A1 and A2 noradrenergic neurons, respectively (Chen et al., 1999; Roland et al., 1999).

Localization of PrRP fibre projections : In the rat brain, a dense network of PrRP-ir fibers was identified in the PVN, SON, DMH/LHA and POA regions of the hypothalamus, but also in the amygdala and area postrema (Iijima et al., 1999; Maruyama et al., 1999; Ibata et al., 2000; Lin, 2008). PrRP-positive neurons from the NTS project to the PVN (Onaka, 2004) where they contact 
anorexigenic oxytocin (Maruyama et al., 1999; Ibata et al., 2000; Yamashita et al., 2013) and corticotrophin-releasing hormone-containing neurons (Maruyama et al., 1999; Matsumoto et al., 1999; Lin et al., 2002). More than 90\% of PrRP neurons are leptin receptor-positive (Ellacott et al., 2002).

Localization of the PrRP receptor: The PrRP receptor is widely distributed in the brain, with high to moderate expression in the VMH, PVN, POA, DMH, AP and NTS consistent with a prominent role in the regulation of food intake and energy homeostasis (Fujii et al., 1999; Roland et al., 1999; Ibata et al., 2000; Lin, 2008). Although such a receptor localization was supported by ISH, RT-PCR and IHC studies, the existence of mismatches between the localization of PrRP-containing nerve fibers and of PrRP-R raised several hypotheses, among which the possibility for PrRP to signal via other receptors (Lin, 2008; Dodd \& Luckman, 2013).

\subsubsection{Effect of PrRPs on food intake}

Accumulating evidence indicates a prominent role of the PrRP system in the stimulation of energy consumption and the reduction of food intake. In fasted rats, i.c.v. administration of PrRP reduces food intake (without impacting water intake) and weight gain, and increases energy expenditure in brown adipose tissue (Lawrence et al., 2000, 2002; Ellacott et al., 2003; Gu et al., 2004). Compared to an i.c.v. application, intra-DMH injection of PrRP promoted an earlier, weaker and longer anorexia in rats while intra-PVN injection had no significant impact on feeding behaviour (Seal et al., 2001).

PrRP-induced hypophagia is abolished in PrRP-R KO mice (Bechtold \& Luckman, 2006) and in the @LETFôrat strain that expresses non functional PrRP receptors (Watanabe et al., 2005). These rats, together with PrRP -/- and PrRP-R -/- null mice, showed late-onset obesity and a hyperphagic phenotype, which depended on feeding experimental paradigms ( $\mathrm{Gu}$ et al., 2004; Watanabe et al., 2005; Bechtold \& Luckman, 2007; Bjursell et al., 2007; Takayanagi et al., 2008).

Genetic ablation of the PrRP system in mice abrogates CCK-mediated satiety (Lawrence et al., 2002; Bechtold \& Luckman, 2006; Takayanagi et al., 2008). Several explanations have been proposed in light of the prominent role exerted by the NTS in satiety regulation, the privileged localization of PrRP neuronal cell bodies, fibers and receptors within this brainstem nucleus and their functional activation by the satiety factor CCK (Lawrence et al., 2002; Dodd \& Luckman, 2013). To sum up, PrRP-induced hypophagia may originate from a reduction in meal size due to an acceleration of satiety signalling.

\subsubsection{Effects of PrRP on energy homeostasis}


Pair-feeding studies suggest that PrRP effect on body weight cannot be attributed solely to its anorectic-like action. Indeed, acute PrRP injections in fasted and satiated rats cause a long lasting hyperthermia, an increase in oxygen consumption and in mRNA levels of the brown adipose tissue factor UCP-1, pointing to an increased energy expenditure (Ellacott et al., 2002, 2003; Lawrence et al., 2004). Noteworthy, animals became rapidly refractory to the anorectic and body weight action of PrRP upon its chronic administration (Ellacott et al., 2003). In marked contrast with the long term effects of leptin (Zhang et al., 1994), tolerance is a phenomenon encountered for many short term acting peptides such as CCK (Crawley \& Beinfeld, 1983), galanin (Smith et al., 1994) or MCH (Rossi et al., 1997). Thus, although leptin and PrRP have similar and additive acute effects (Ellacott et al., 2002), the latter peptide may affect energy homeostasis only in the short to medium term.

PrRP -/- and PrRP-R -/- male mice show no significant differences in oxygen consumption or core body temperature in basal conditions, as compared to wild-type animals (Bjursell et al., 2007; Takayanagi et al., 2008). However, the increase in $\mathrm{O}_{2}$ consumption was reduced in $\mathrm{PrRP}-/-$ mice after stressful stimuli (Takayanagi \& Onaka, 2010). As former studies showed that emotional stress and nociceptive stimuli were able to activate medullary and hypothalamic neurons (Maruyama et al., 2001) and to up-regulate their content in PrRP mRNA (Mera et al., 2006), PrRP may regulate energy expenditure in response to stress conditions (Onaka et al., 2010; Maniscalco et al., 2012).

Finally, the possibility that PrRP mediates central anorectic effects through a receptor distinct from PrRP-R has been evoked (Ellacott et al., 2005; Onaka et al., 2010; Dodd \& Luckman, 2013). The NPFF2 receptor is a putative candidate as it displays a high affinity for PrRPs (Table 3), a significant expression in hypothalamic areas, and mediates a similar response (reduction in food intake) following i.c.v NPFF injection (Section 7.3).

\subsubsection{Effects of metabolic signals on PrRP/PrRP-R expression}

PrRP-R -/- null mice exhibit a reduced glucose tolerance and a significant increase in insulin and leptin levels (Gu et al., 2004). The anorectic response to leptin is impaired in PrRP and PrRP-R KO mice (Takayanagi et al., 2008; Dodd \& Luckman, 2013), an observation which could be related to the fact that most PrRP-containing neurons in the hypothalamus and brainstem express leptin receptors (Ellacott et al., 2002). PrRP mRNA expression in the NTS, DMH and VMH is down regulated in situations of food restriction that correlate with reduced signalling of the adiposederived hormone leptin (Lawrence et al., 2000; Ellacott et al., 2002). Although the functional interplay between leptin and PrRP has not been fully elucidated, both peptides may mediate satiety signalling from the adipose tissues.

H7.5. Kisspeptins and the Kiss1 receptor 


\subsection{Kisspeptins and the Kiss 1 receptor}

\subsubsection{Distribution of kisspeptins and their receptor in peripheral organs and CNS areas involved in}

energy homeostasis.

A schematic presentation of the localization of Kiss-containing cell bodies and of Kiss1R in the rodent brain, which supports the involvement of this RF-amide system in feeding behaviour, is shown Figure 7. The neuroanatomy of the kisspeptin system in mammalian brain has been described in many papers (Clarkson et al., 2009; Herbison et al., 2010; Smith, 2013) along with potential detection artefacts (Mikkelsen \& Simonneaux, 2009).

Localization of kisspeptin-containing neurons and cell bodies : KiSS-1 mRNA was detected in several peripheral organs involved in feeding processes such as mouse and human pancreatic cells (Ohtaki et al., 2001; Hauge-Evans et al., 2006), mouse and human liver (Ohtaki et al., 2001; Song et al., 2014) and rat fat tissue (Brown et al., 2008). KiSS-1 immunoreactivity was also detected in mouse and human plasma (Song et al., 2014).

Within the mammalian brain, kisppeptin-containing neuronal cell bodies essentially locate within two hypothalamic regions: the arcuate nucleus (ARC) and the periventricular area of the third ventricle (referred to as RP3V), a continuum of cells that extends from the anteroventral periventricular nucleus (AVPV) to the preoptic (POA) area. ISH and IHC studies have consistently highlighted these kisspeptin cell populations in every mammalian species examined to date, including mice (Gottsch et al., 2004; Clarkson \& Herbison, 2006), rat (Kinoshita et al., 2005; Smith et al., 2006b; Mikkelsen \& Simonneaux, 2009; Xu et al., 2012), hamster (Revel et al., 2006; Greives et al., 2007), ovine (Pompolo et al., 2006; Franceschini et al., 2006; Goodman et al., 2007), monkey and man (Shahab et al., 2005; Rometo et al., 2007; Ramaswamy et al., 2008). Kisspeptin-ir and KiSS-1 mRNA-expressing cell populations were also detected in the amygdala (Kim et al., 2011; Xu et al., 2012) and between the dorsomedial (DMH) and ventral (VMH) hypothalamic nuclei (Dun et al., 2003; Brailoiu et al., 2005; Clarkson \& Herbison, 2006; Franceschini et al., 2006; Bosch et al., 2012). A broad distribution profile for KiSS-1 neurons was described using a Kiss1Cre/ $\beta$-galactosidase transgenic mouse model (Cravo et al., 2011) but the specificity of this reporter system awaits further confirmation.

One cannot exclude that early IHC data might have been limited by cross-reactivity of kisspeptin antibodies with other RF-amide peptides (Mikkelsen \& Simonneaux, 2009). More recently, using a well-characterized kisspetin antibody validated on brain tissue sections of Kiss1-KO mice, Clarkson and coworkers provided a detailed description of KiSS-1-ir cell bodies and fibres in female mouse brain and confirmed the existence of three main populations of kisspeptin-producing cells located in the RP3V, the ARC and in the DMH at a very low density (Clarkson et al., 2009). To note that the 
relative distribution of kisspeptin neurons within these hypothalamic regions vary between species and gender, with the AVPV nucleus displaying a marked sexual dimorphism in rodents (Section 8.5.1.).

Localization of kisspeptin fibre projections : Abundant neuronal Kiss-ir fibres have been found in hypothalamic areas such as the ARC, POA, paraventricular (PVN) and the DMH nuclei in rodents, while low-density fibres were located in discrete extra-hypothalamic regions (Brailoiu et al., 2005; Kinoshita et al., 2005; Clarkson \& Herbison, 2006; Franceschini et al., 2006; d'Anglemont de Tassigny et al., 2008; Ramaswamy et al., 2008; Clarkson et al., 2009). Using anterograde and retrograde tracing techniques to establish the projection pattern of kisspeptin cells in mouse brain, Yeo and Herbison confirmed the existence of wide projections of neurons originating from the ARC and the RP3V regions onto DMH, PVN and POA hypothalamic nuclei (Yeo \& Herbison, 2011). ARC projections to associated limbic structures such as the lateral hypothalamic area (LHA) were also identified.

Localization of Kiss1 receptors : Kiss1R transcripts are present in various peripheral tissues involved in energy homeostasis such as the pancreas, the adipose tissue and possibly the stomach and the intestine (Kotani et al., 2001a; Muir et al., 2001; Ohtaki et al., 2001; Hauge-Evans et al., 2006; Song et al., 2014; Chen et al., 2014).

In rat brain, Kiss1R mRNA was detected by ISH in several hypothalamic nuclei (ARC, DMH, LHA and POA) and in mesocorticolimbic areas such as the nucleus accumbens (NAc), ventral tegmental area (VTA), amygdala (Amd), striatum and orbito frontal (OFC) cortex (Lee et al., 1999). All latter structures have been involved in food rewarding processes (Gao \& Horvath, 2008; Lenard \& Berthoud, 2008; Dardeno et al., 2010; Volkow et al., 2011).

The use of a transgenic Kiss1R LacZ knock-in mouse provided a detailed mapping of Kiss1R gene expression that fairly well fitted the distribution of receptor mRNAs in rat brain (Herbison et al., 2010). Noticeable exceptions were the lack of X-gal labelling in the amygdala and VTA areas and especially in the ARC that contains abundant kisspeptin fibers in mouse and rat (Clarkson et al., 2009; Mikkelsen \& Simonneaux, 2009; Yeo \& Herbison, 2011) and expresses functional Kiss1 receptor as shown by electrophysiological studies performed on mouse brain (Fu \& van den Pol, 2010).

\subsubsection{Kisspeptin-mediated control of food intake and energy balance}

Despite their óstrategicôlocation within hypothalamic areas and peripheral tissues (pancreas and adipose tissue) involved in energy homeostasis, endogenous kisspeptins and their receptor have long been considered as poorly effective in controlling body weight or food consumption. Indeed, initial studies failed to evidence central effects of kisspeptins on food intake in ad libitum-fed or 
pre-fasted rats (Navarro et al., 2004; Thompson et al., 2004; Castellano et al., 2005). This was later confirmed in ewes that received food and water ad libitum (Clarke et al., 2012). Kiss1R KO mice displayed no obvious phenotypes related to feeding behaviour as well (Seminara et al., 2003). However, Kisspeptin-10 (i.c.v.) was reported to increase meal intervals in mice, while meal size remained unchanged (Stengel et al., 2011), and to slightly reduce food intake and body weight of female rats during the pubertal period (Sahin et al., 2015).

In contrast to former reports, convincing evidence was provided, at least in Kiss1R KO female mice up to 8-10 weeks old, for a regulatory role of kisppeptins in body weight, adiposity, energy balance and glucose homeostasis (Tolson et al., 2014). Both male and female Kiss1R -/- mice displayed an increased fat mass along with marked hyperleptinemia. Only females showed a significant increase in body weight, higher basal glucose levels and altered glucose tolerance relative to wild-type or heterozygous females. Surprisingly, KO females were hypophagic and their obesity likely resulted from reduced energy expenditure and locomotor activity. Thus, adult female Kiss1R KO mice display a metabolic and diabetic phenotype but further experiments are needed to dissect the contribution of strain, gender and dependency on kisspeptin signalling (ligand versus receptor). Indeed, a previous comparison of male and female Kiss1R KO mice pointed to a slight decrease in body weight of males at 9-12 weeks, without noticeable variation in females (Lapatto et al., 2007; Garcia-Galiano et al., 2012). KiSS-1 KO female mice were significantly smaller than their wild-type littermates at two months of age (d'Anglemont de Tassigny et al., 2007) while another study found no feeding-related phenotype for KiSS-1 KO mice of either sex (Lapatto et al., 2007).

The work by Tolson and collaborators (Tolson et al., 2014) raises also several questions regarding the interaction of metabolic/diabetic cues and the role of kisspeptin signalling in the brain and/or the periphery. Both in vitro and in vivo kisspeptin treatments have been found to enhance glucose-induced insulin secretion (Hauge-Evans et al., 2006; Wahab et al., 2011), supporting a role of kisspeptins in pancreatic 6 cell function. Such a possibility may underlie the impaired glucose tolerance of Kiss1R KO female mice (Tolson et al., 2014). However, several studies already reported an inhibition by kisspeptins of glucose-induced insulin secretion from $\beta$ pancreatic MIN6 (Hauge-Evans et al., 2006) and NIT (Chen et al., 2014) cell lines, rat perfused pancreas (Silvestre et al., 2008) and mouse pancreatic islets (Vikman \& Ahren, 2009; Chen et al., 2014). Chen and coworkers investigated into more detail the mechanisms by which kisspeptin affects insulin release and evidenced a cross-talk between Kiss1R signaling and the transcriptional factor Isl-1 (Chen et al., 2014). Kiss1R and Isl-1 were found to be coexpressed in mouse pancreatic islets and in NIT cells. Both in vitro and in vivo experiments, taking advantage of an Isl-1-inducible KO mouse model, revealed that Kisspeptin-54 inhibited insulin secretion by lowering Isl-1 expression and 
promotor activity of the insulin gene. Conversely, a feedback loop driven by insulin negatively regulated KiSS-1 mRNA levels and kisspeptin concentration in mouse NIT and pancreatic cells. This study also identified two distinct signaling pathways responsible for kisspeptin and insulin effects. Most interestingly, the discovery of a hormonal kisspeptin circuit between the liver and the endocrine pancreas highlighted a link between hyperglucagonemia, increased hepatic kisspeptin production and impaired glucose-stimulated insulin secretion from $\beta$ pancreatic cells in type 2 diabetes (Song et al., 2014). Morevover, and in contrast with Tolson and coworkersô findings (Tolson et al., 2014), liver-specific KiSS-1 knockdown in mice submitted to various metabolic alterations was found to increase glucose-induced insulin secretion and to improve glucose tolerance (Song et al., 2014).

\subsubsection{Impact of energy status on expression of the kisspeptin system}

As the metabolic status is a known regulator of reproductive activity (Schneider, 2004; Schneider et al., 2013), much attention has been paid to the kisspeptinergic system as a link between energy balance and fertility (Castellano et al., 2010a; Navarro \& Tena-Sempere, 2012; Parhar et al., 2012; Wahab et al., 2013; De Bond \& Smith, 2014). Indeed, the expression and activity of kisspeptins and their receptor are highly sensitive to metabolic cues and regulatory factors. The next section will focus on the impact of negative (fasting, caloric restriction, lactation) and positive (high fat diet, obesity, diabetes) energy balance states on the KiSS-1/Kiss1R system as both under- and over-nutrition result in reproductive dysfunction.

Low energy balance : Acute fasting was consistently associated with a significant reduction of hypothalamic KiSS-1 mRNA levels in pre-pubertal male and female rats (Castellano et al., 2005) and in female (Brown et al., 2008; Kalamatianos et al., 2008; Matsuzaki et al., 2011) and male (Luque et al., 2007) adult rats. Similar responses to low food supply were reported in monkeys (Wahab et al., 2011). Chronic food restriction also down-regulated KiSS-1 expression in ovariectomized (OVX) ewes (Backholer et al., 2010) and in post-pubertal gilts (Zhou et al., 2014).

Other conditions of negative energy balance, such as lactation (Yamada et al., 2007; Xu et al., 2009), chronic blockade of mTOR in ovariectomized (OVX) rats (Roa et al., 2009), caloric restriction (True et al., 2011) or metabolic stress, such as streptozotocin-induced diabetes mellitus (Castellano et al., 2006b; Castellano et al., 2009a) or acute inflammation evoked by systemic injection of lipopolysaccharide (Castellano et al., 2010b), also pointed to a close relationship between altered gonadotropic function and decreased hypothalamic KiSS-1 mRNA levels.

The reduction in hypothalamic KiSS-1 expression concerned both the ARC and AVPV nuclei in rodents (Castellano et al., 2005; Luque et al., 2007; True et al., 2011) and pig (Zhou et al., 2014) or the ARC and POA in ewe brain (Backholer et al., 2010). However, KiSS-1 expression was reported 
to be more severely impaired in the AVPV of fasted OVX rats (Kalamatianos et al., 2008) whereas KiSS-1 mRNA levels were selectively decreased in the ARC of gonadally intact adult female rats (Matsuzaki et al., 2011) or of OVX rats following chronic mTOR blockade (Roa et al., 2009). Thus, these two KiSS-1 neuronal populations probably differentially contribute to the coupling of energy balance with gonadal function.

The impact of a low energy balance on hypothalamic Kiss1R expression appears also highly variable: an increase in Kiss1R expression was reported in rats (Castellano et al., 2005), a decrease was observed both in male adult rats (Luque et al., 2007) and in nutrient-restricted gilts, specifically in the ARC nucleus (Zhou et al., 2014), while no significant expression changes occurred neither in the ARC nor in the AVPV of fasted female rats (Matsuzaki et al., 2011).

The functional relevance of former observations was confirmed by studies on undernourished female rats (Castellano et al., 2005) and on uncontrolled diabetic male and female rats (Castellano et al., 2006b, 2009a) where kisspeptin-10 injections partially rescued hypogonadotropism and reproductive defects. Similarly, in women suffering from hypothalamic amenorrhea (often associated with negative energy balance), repeated administration of kisspeptin-54 substantially improved their gonadotropic function (Jayasena et al., 2010). Interestingly, acute fasting and caloric restriction in pubertal female rats not only decreased KiSS-1 mRNA in the ARC and AVPV but also inhibited the hypothalamic expression of the neurokinin B (NKB) gene and its NK3 receptor (Navarro et al., 2012). Given the coexpression of NKB and KiSS-1 in ARC neurons (Goodman et al., 2007; Ramaswamy et al., 2010) and the fact that delayed female puberty may also be partially prevented by repeated administration of NKB (Navarro et al., 2012), there is a possibility for NKB and KiSS-1 signalling to cooperate in the metabolic control of puberty (Navarro, 2013).

Positive energy balance : Obesity and diabetes are paradigms of altered energy homeostasis in which more than sufficient reserves of metabolic fuels are available, but the body cannot use them. Although they are frequently associated to lower fertility and altered puberty onset, the effects of persistent overweight on the expression and activity of the hypothalamic kisspeptinergic system have been much less investigated than those of energy deficit (Ahmed et al., 2009; Loret de Mola, 2009; Biro \& Wien, 2010; Rachon \& Teede, 2010).

In ob/ob mouse lacking the leptin gene, kisspeptin signalling is disrupted as revealed by a decrease in KiSS-1 gene expression (Smith et al., 2006a), especially within the ARC nucleus (Quennell et al., 2011). Noteworthy, exogenous leptin (but not caloric restriction) is able to reverse adverse effects of obesity on reproduction in males and females (Mounzih et al., 1997; Cleary et al., 2001).

Studies on DBA/2J mice, prone to high fat diet-induced infertility, evidenced a marked reduction in KiSS-1 mRNA levels in the ARC and RP3V and of Kiss-ir neurons in the AVPV (Quennell et 
al., 2011). Conversely, pre-pubescent gilts fed a high fat diet showed an early attainment of puberty and increased KiSS-1 and Kiss1R mRNAs expression in the AVPV (Zhuo et al., 2014). Metabolic programming of puberty onset related to post-natal over/under feeding and differential hypothalamic KiSS-1 expression has recently been proposed (Castellano et al., 2011).

Streptozotocin (STZ)-treated rats is a widely used model of type 1 diabetes, with the destruction of pancreatic islets leading to hypoinsulinemia and hyperglycemia (Arison et al., 1967). Such diabetic animals are characterized by a hypogonadotropic hypogonadism (Spindler-Vomachka \& Johnson, 1985; Sexton \& Jarow, 1997), with normal LH responses to kisspeptin, low levels in KiSS-1 mRNAs but normal Kiss1R expression in the hypothalamus (Castellano et al., 2006b). Gonadotropin deficiency in this diabetic model most likely occured upstream of GnRH neurons, with hypoleptinemia being a critical factor for reducing Kiss expression and subsequent excitatory input to GnRH neurons (Castellano et al., 2006b; Luque et al., 2007; Quennell et al., 2011).

Most interestingly, KiSS-1 was recently uncovered as a causal link between early dysregulated glucagon secretion by pancreatic $\alpha$ cells and impaired glucose-induced insulin secretion from $\beta$ cells in type 2 diabetes (Song et al., 2014). Glucagon was found to stimulate KiSS-1 production in the liver from human diabetic patients and diabetic mouse models. Importantly, KiSS-1 knockdown in the liver of hyperglucagonemic, glucose-intolerant, high-fat-diet fed, and leptin insensitive db/db mice increased glucose-induced insulin secretion and improved glucose tolerance.

In the adipose tissue, the KiSS-1 gene is regulated by sex steroids and the nutritional status (Brown et al., 2008). In contrast with findings in the hypothalamus, food restriction increased Kiss mRNA levels in the adipose tissue of male and female rats, while rats fed a high fat diet or obese Zucker rats showed a lower KiSS-1 expression in this tissue. Neonatal excitotoxic lesion of the ARC did not affect KiSS-1 mRNA levels in fat tissue, suggesting that kisspeptins may function as local regulators of adipocyte function.

Altogether, much data emphasize the importance of the kisspeptin neuronal network in the integration of metabolic signals to control puberty and reproduction. In the hypothalamus, the kisspeptin system may operate as a metabolic sensor that transmits nutritional relevant information to the HPG axis to adjust reproductive activity with available energy reserves. In fact, an array of metabolic signals and neuropeptides are involved in the maintenance of energy balance and reproductive processes (Schneider, 2004; Schneider et al., 2013) and the interplay of leptin, but also ghrelin and adiponectin, as well as of NPY/POMC neuropeptides with the kisspeptinergic system still receives considerable attention (Castellano et al., 2009b; True et al., 2011; Evans \& Anderson, 2012; Garcia-Garcia, 2012; Sanchez-Garrido \& Tena-Sempere, 2013; De Bond \& Smith, 2014). 


\subsection{QRFPs and their QRFP receptor}

7.6.1. Distribution of QRFPs and their receptor in peripheral organs and CNS areas involved in energy homeostasis.

A schematic presentation of the localization of QRFP-containing cell bodies and of QRFP$\mathrm{R} 1 / \mathrm{R} 2$ receptors in rodent brain, with reference to regions involved in the regulation of energy balance, is shown Figure 7.

Localization of QRFP-containing neurons : The expression and localization of QRFP-26 and QRFP-43 peptides and of their transcripts have been assessed in human, rat and mouse tissues using RT-qPCR, ISH and IHC.

At the periphery, significant levels of QRFP transcripts were found in the human retina (Jiang et al JBC 2003), rat optic nerve (Fukusumi et al., 2003) and mouse eye (Takayasu et al., 2006). RTqPCR and IHC experiments using antibodies against QRFP-26 confirmed the expression of QRFPs in human gut and pancreatic islets as well as in mouse gut, liver, endocrine pancreas and MIN6 insulinoma cells (Jiang et al., 2003; Prevost et al., 2015). QRFPs mRNA are also expressed in mouse adipose tissue and 3T3-L1 adipocytes (Zhang et al., 2007; Mulumba et al., 2010; Jossart et al., 2014) and in murine macrophages (Jossart et al., 2014). Circulating levels of QRFP-26 were also measured in human plasma by using a radio-immunoassay (Galusca et al., 2012; Prevost et al., 2015).

Within the CNS, ISH studies indicated that QRFP-26 and QRFP-43 were primarily expressed in the hypothalamus, within the LHA, the periventricular (PeV), VMH and ARC nuclei in rat (Chartrel et al., 2003; Kampe et al., 2006), mouse (Takayasu et al., 2006) and human (Bruzzone et al., 2006) brain. QRFP mRNA also displays a species-dependent distribution : in the human brain, it is abundant in the cerebellum and the medulla, with a moderate expression in the caudate putamen, VTA and hypothalamus while in mouse brain, it concentrates in the hypothalamus and the cortex (Jiang et al., 2003).

Localization of the QRFP receptor in the CNS and peripheral tissues : The distribution of QRFP-R in mammalian tissues is essentially from RT-qPCR, in situ hybridization and radioligand binding assays.

Fairly high levels of QRFP receptor mRNA were found in the human retina (Jiang et al., 2003) whereas lower levels were detected in rat and mouse eye (Fukusumi et al., 2003; Takayasu et al., 2006). QRFP-R mRNA was also detected in human omental adipocytes and in murine 3T3-L1 adipocytes (Mulumba et al., 2010) as well as in mouse adipose tissue and MIN6 insulinoma cells (Prevost et al., 2015). The presence of QRFP-R immunoreactivity in human pancreatic sections has been recently reported (Prevost et al., 2015). 
The QRFP receptor is primarily expressed in the CNS where it displays a much wider distribution than QRFP peptides. Within the human brain, QRFP-R mRNA is moderately enriched in the hypothalamus and the cortex, lower levels are found in the amygdala and VTA (Jiang et al., 2003). The localization of QRFP receptors in mouse (Jiang et al., 2003) and rat (Fukusumi et al., 2003) brain, first examined through RT-PCR and ISH experiments, was further detailed thanks to a comparative study based on ISH and autoradiographic mapping of [ $\left.{ }^{125} \mathrm{I}\right]-26 \mathrm{RFa}$ binding sites in rat brain (Bruzzone et al., 2007). The existence of some regions enriched in 26RFa binding sites but deprived of QRFP mRNA as well as inconstancies in the distribution patterns of the QRFP-R in rat brain (Fukusumi et al., 2003; Bruzzone et al., 2007) were, at least in part, explained by the presence of two receptor orthologs QRFP-R1 and QRFP-R2 in rodent brain (mouse GPR103A/B; (Takayasu et al., 2006) and rat QRFP-r1/2, (Kampe et al., 2006)). Given the high homology and close pharmacological properties of these receptors (Section 3.5.4.), the possibility that riboprobes and/or $\left[{ }^{125} \mathrm{I}\right]-26 \mathrm{RFa}$ actually labelled a mixture of GPR103A/B receptors in rat brain could not be excluded.

Although a systematic comparison of the distribution of GPR103A/B receptor transcripts was undertaken in mouse brain (Takayasu et al., 2006), data from Fukusumi and coworkers (Fukusumi et al., 2003) were taken as the reference pattern for QRFP-r1 expression in rat brain (Kampe et al., 2006). Several qualitative and quantitative discrepancies regarding the distribution of the GPR103B (QRFP-r2) receptor mRNA in mouse and rat brain (Kampe et al., 2006) still merit further examination. With reference to regions regulating energy balance, a consensus view reconcilying both rodent species may be proposed as follows : QRFP-R1 mRNA is found in the olfactory bulb, the cortex-amygdala transition zone, in various hypothalamic areas (ARC, VMH, PVN), in the NTS (Fukusumi et al., 2003; Takayasu et al., 2006) and possibly in the parabrachial (PB) and dorsal motor vagal (DMX) nuclei (Bruzzone et al., 2007); QRFP-R2 mRNA is mainly detected in the nucleus accumbens (NAc) shell and caudate putamen (CP), in the VTA, and in some regions (POA, LHA, PVN) of the hypothalamus (Kampe et al., 2006; Takayasu et al., 2006). Thus, QRFP receptor subtypes differentially distribute in rodent brain, with only very little overlap.

\subsubsection{QRFP-mediated control of food intake}

First evidence for an orexigenic effect of QRFPs was provided by studies performed on mice, either freely fed (do Rego et al., 2006; Takayasu et al., 2006), submitted to a moderate high fat diet (Moriya et al., 2006) or to partial food restriction (Chartrel et al., 2003). Acute i.c.v. injection of QRFPs, during early light phase, induced a dose-dependent increase in food intake, in locomotor activity and in oxygen consumption (do Rego et al., 2006; Takayasu et al., 2006). 
Chronic administration of QRFPs had no significant impact on body weight in mice under standard chow diet, in marked contrast with NPY which led to obesity (Moriya et al., 2006; Takayasu et al., 2006). The body weight of QRFP-R1 null mice -which suffer from a severe osteopenia- was indistinguishable from that of their wild-type littermates (Baribault et al., 2006), although no indication was given on how the animals were housed or fed. Thus, QRFP anabolic action may be counteracted by an increase in energy expenditure. In mice fed a moderately high fat diet, the continuous infusion of QRFPs caused a transient hyperphagia and a net increase in body weight (Moriya et al., 2006). However, pair-fed control and treated mice experienced a similar weight gain while QRFP-43 clearly increased fat mass. Thus, QRFPs may cause metabolic changes independently of their orexigenic effect.

The situation was more contrasted in rats, in which QRFPs were first reported to have no significant effects on the intake of standard low fat chow diet (Kampe et al., 2006; Patel et al., 2008). Subsequent studies evidenced an orexigenic effect of QRFP-26 and QRFP-43 only when rats were fed a high fat diet (Primeaux et al., 2008) or food-restricted (Lectez et al., 2009). Thus, QRFPs may increase the motivation to eat, drive preference for highly palatable food rich in fat and sucrose and/or regulate reward systems in the brain (Primeaux et al., 2013).

The possibility for QRFP-26 and QRFP-43 to mediate their orexigenic activity in part through the modulation of the hypothalamic NPY/POMC system (Takayasu et al., 2006) was confirmed by a series of converging observations in rats (Lectez et al., 2009). These data support the view that QRFP neurons originating from the VMH and LHA project onto NPY-ergic neurons within the ARC. QRFP-26 release and subsequent receptor activation increase food intake by stimulating the expression and release of NPY which in turn inhibits the firing of POMC neurons by activating Y1 and Y5 NPY receptors (Lectez et al., 2009).

Searching for putative interactions of QRFPs with other orexigenic peptide networks pointed to orexin as a plausible candidate. QRFP-promoted food intake in orexin -/- mice was similar to that found in wild-type mice (Takayasu et al., 2006). In genetically obese mice models with leptin insufficiency (ob/ob) or insensitivity ( $\mathrm{db} / \mathrm{db}$ ), orexin expression was down-regulated (Yamamoto et al., 2000) while prepro-QRFP transcript was up-regulated (Takayasu et al., 2006). Thus, QRFP and orexin probably exert orexigenic effects through independent signalling pathways.

\subsubsection{QRFPs effects on energy homeostasis and adipose metabolism.}

Acute i.c.v. administration of QRFP in mice significantly increased their locomotor activity during the light and dark periods (do Rego et al., 2006; Takayasu et al., 2006). Oxygen consumption, taken as a index of metabolic rate, was also enhanced (Takayasu et al., 2006). Pairfeeding studies conducted under moderate fat diet indicated that mice chronically treated with 
QRFPs did not display marked differences in locomotor activity. However, lowered rectal temperature and expression of the brown adipose tissue uncoupling protein-1 (UCP-1) indicated a decreasing energy expenditure (Moriya et al., 2006). These QRFP-treated mice also exhibited a higher fat mass and increased plasmatic concentrations of insulin, leptin, cholesterol, free fatty acids and triglycerides (Moriya et al., 2006).

The inhibition by QRFP-26 of glucose-induced insulin secretion in rat perfused pancreas (Egido et al., 2007) was observed in rat pancreatic INS-1E- $\beta$ cells and in human pancreatic islets (Granata et al., 2014). In the latter study however, QRFP-26 and QRFP-43 were found to exert opposite effects on insulin secretion, mediated via $\mathrm{G}_{\mathrm{i} / \mathrm{o}}$ inhibition or $\mathrm{G}_{\mathrm{s}}$ stimulation of cAMP production, respectively. QRFP receptor silencing blocked the QRFP-43 insulinotropic effect in INS-1E- $\beta$ cells, leaving the QRFP-26 insulinostatic response unaffected. In marked contrast with these findings, Prévost and collaborators showed that QRFP-26 was able to stimulate insulin release from MIN6 insulinoma cells, via the activation of endogenous QRFP receptors (Prevost et al., 2015). As MIN6 cells do not express the NPFF2 receptor, the possibility for QRFP-26 to mediate an inhibition of insulin release from INS-1E- $\beta$ cells and pancreatic islets via activation of NPFF2R has been evoked (Prevost et al., 2015). Most interestingly, QRFP-26 was proposed to play an incretin-like role in the regulation of glucose homeostasis : following glucose ingestion, it is secreted from the gut into the blood to reach pancreatic $\beta$ cells (where it stimulates insulin secretion) and insulin-sensitive tissues, such as the striated muscle and the liver (where it may possibly favor glucose uptake), in order to adjust glycemia levels (Prevost et al., 2015).

The role of QRFPs and of their QRFP receptor in adipogenesis and lipid metabolism has been investigated in differentiated murine 3T3-L1 adipocyte cells (Mulumba et al., 2010). These cells (which express the QRFP precursor and the QRFP-R2 receptor) responded to a QRFP-26 or QRFP43 application by a pronounced increase in their triglyceride content and in fatty acid uptake and by up-regulating various genes involved in lipid storage. These responses as well as the inhibition by QRFP-26 or QRFP-43 of isoproterenol-induced lipolysis were blocked following QRFP-R2 knockdown in 3T3-L1 cells. Thus, in addition to their central orexigenic effect, QRFPs may act as peripheral adipogenic factors to regulate fatty acid uptake and fat storage, via activation of the QRFP-R2.

\subsubsection{Impact of energy status on the expression of QRFPs and their receptor}

Obese individuals and human patients with type- 1 and type- 2 diabetes, under fasting conditions, show marked elevation in their QRFP-26 plasma levels (Prevost et al., 2015). In fasted mice, as compared to fed control animals, as well as in genetically obese mice characterized by leptin insufficiency (ob/ob) or leptin insensitivity (db/db) fed on an ad libitum chow diet, hypothalamic 
prepro-QRFP mRNA levels are up-regulated (Takayasu et al., 2006). On the other hand, mice fed a moderately high fat diet and chronically treated with QRFP-43 display a marked hyperleptinemia, including in QRFP/pair-fed groups (Moriya et al., 2006).

Rats consuming high fat diet for 3 weeks gained more weight and fat mass, as compared to animals fed a low fat diet, and showed increased prepro-QRFP mRNA levels in the VMH/ARC region of the hypothalamus while QRFP-R expression remained unchanged (Primeaux et al., 2008). Another study systematically examined the influence of dietary composition on hypothalamic QRFP-43 content (Beck \& Richy, 2009). Rats fed ad libitum for 8 weeks with either standard or high-fat diet did not display differences in body weight or total energy intake, indicating that rats adapted their quantity of food to counterbalance the higher energy density of high fat diet. However, diet composition strongly impacted hypothalamic QRFP-43 expression, as it became undetectable in the VMH of rats submitted to high fat diet. Thus, a decrease in the QRFP orexigenic tone may contribute to the normalization of caloric intake in rats fed a high fat diet. Most interestingly, QRFP-43 levels in the VMH (but not in the LHA) inversely correlated with leptin plasma levels which in turn significantly correlated with diet adipogenic index, as expected (Barsh $\&$ Schwartz, 2002). Leptin exerts its anorexigenic activity mainly via the NPY/POMC system in the ARC (Satoh et al., 1997; Tang-Christensen et al., 1999) and recent studies already pointed to the importance of the QRFP/leptin balance in the regulation of that system (Lectez et al., 2009). The data from Beck and Richy confirmed the role of leptin in the VMH where it may regulate, according to its plasma levels, the expression of the orexigenic QRFP peptides (Beck \& Richy, 2009).

The nutritional or energy status also affects QRFP expression in peripheral tissues. Indeed, in a mouse model of diet-induced obesity, the expression of QRFP-R2 was strikingly increased in metabolically active fat tissues whereas QRFP gene expression was decreased, most probably by pro-inflammatory cytokines such as TNF- $\alpha$ (Mulumba et al., 2010).

Jossart and collaborators uncovered the expression and secretion of QRFP by macrophages, and its paracrine role between macrophages and adipocytes with a pro-adipogenic effect (Jossart et al., 2014). Down-regulation of QRFP expression in adipocytes and macrophages was found to primarily depend on IFN- $\beta$ levels which were up-regulated in macrophages following LPS stimulation and elevated in obese mice with metabolic endotoxemia. To note that endotoxins play an important role in the initiation of insulin resistance in obesity (Cani et al., 2008) and that macrophages participate to the development of obese adipose tissue inflammation (Anderson et al., 2010). More generally, this study highlighted an autocrine/paracrine function for QRFP and its contribution to a switch in the obese adipose tissue from a proadipogenic to a pro-inflammatory 
state. Therefore, QRFP was proposed as a potential biomarker for metabolic endotoxemia in adipose tissue (Jossart et al., 2014).

In line with consistent observations indicating that QRFPs had no effect on food intake when injected during the dark phase in mice (Takayasu et al., 2006) and rats (Kampe et al., 2006), QRFP26 was recently identified as a circulating hormone submitted to circadian variations in humans (Galusca et al., 2012). In healthy women, QRFP-26 plasma levels were high and stable during the morning, dramatically felt at noon and remained low until next early morning. Anorectic patients revealed an alteration of the circadian rhythmicity and a marked increase in mean circulating QRFP-26 levels together with those of ghrelin. In addition to lower BMI and fat mass, these subjects had also lower leptin and $17 \beta$-estradiol levels than the control group. These data may reflect an adaptive mechanism of the organism to promote energy intake and to increase fat stores in response to undernutrition.

To conclude this section, all RF-amide neuropeptides and their receptors contribute in many ways to the regulation of food intake and energy balance in Mammals. Despite the fact that their implication in feeding behaviour has been conserved throughout evolution (Dockray, 2004; Bechtold \& Luckman, 2007), it is still difficult to ascertain whether it represents their primary function. Nowadays, RF-amides may be better regarded as one of the numerous peptidergic systems that cooperate to finely tune energy homeostasis, both at the central and peripheral levels. In addition to the difficulties associated to deciphering the proper role of each RF-amide/receptor pair in the regulation of complex physiological functions (appetite, energy metabolism or food reward, among others) as well as in pathological conditions such as obesity and diabetes, the promiscuous binding properties of NPFF1 and NPFF2 receptors still represent a major drawback that should be soon relieved with the development of highly selective pharmacological tools.

\section{Mammalian RF-amide peptides and their receptors in reproduction}

\subsection{Control of reproduction and HPG axis in Mammals}

The control of reproduction in Mammals is governed by the hypothalamo-pituitary-gonadal (HPG) axis and by complex positive and negative steroidal feedback from the periphery. Identified in 1977 by the Medicine Nobel Laureates Roger Guillemin and Andrew V. Schally, the Gonadotropin-Releasing Hormone $(\mathrm{GnRH})$ released by scattered hypothalamic cells represents the main positive regulator of the reproductive system (Matsuo et al., 1971; Burgus et al., 1972). GnRH neurons are mainly located in the preoptic area (POA) and massively project to the median 
eminence (Hahn \& Coen, 2006). Triggered by gonadal steroids, the pulsatile secretion of GnRH into the portal blood enhances the synthesis and pulsatile secretion of LH (Luteinizing Hormone) and FSH (Follicle Stimulating Hormone) gonadotropins from the anterior pituitary (Plant, 2015). In females, positive and negative steroidal feedback on GnRH neurons finely tune LH release. During the almost entire cycle period, negative feedback mechanisms manage the pulsatile secretion of LH allowing follicular development and steroidogenesis (Andreu et al., 1998 ; Jones \& Hsueh, 1981). However, during proestrus, the LH surge preceeding ovulation is triggered by a positive feedback (Moenter et al., 2003) in different rodent species but not in primates (Yamaji et al., 1971 ; Karsch et al., 1973 ; Knobil, 1974).

Among multiple modulators of reproduction, RF-amide peptides are involved in the control of the HPG axis. Notably, Kisspeptin and its receptor are now well described as potent positive regulators of mammalian GnRH neurons. On the other hand, although the precise role of the RFRP3/NPFF1R system is not entirely understood, especially in Mammals, several studies highlight its subtle negative effect under special conditions.

\subsection{The RFRPS/NPFF1R system in the regulation of reproductive axis}

It is important to keep in mind that depending on the publications, RFRP-1 or RFRP-3 are considered mammalian orthologs of GnIH (GnRH Inhibitory Hormone) which represents the main GnRH inhibitory system in birds (Tsutsui et al., 2000; Tsutsui et al., 2010). RFRP-1 is now considered the structural ortholog, whereas RFRP-3 is considered the functional ortholog of avian GnIH (Clarke et al., 2008; Pineda et al., 2010a; Smith \& Clarke, 2010; Tsutsui et al., 2012; Ubuka et al., 2012b). This explains why most studies in Mammals focused on RFRP-3.

\subsubsection{Localization of RFRPs and their NPFF1 receptor in reproductive pathways}

The distribution of RFRP peptides and receptor in brain regions involved in the control of reproduction in rats and mice are highlighted in Figure 8.

RFRP peptides are mainly expressed within the CNS (Chartrel et al., 2006; Tsutsui et al., 2009; Tsutsui et al., 2010). In situ hybridization studies allowed to detect RFRP mRNA in the dorsomedial hypothalamic nucleus (DMH) in mouse and hamster brain (Kriegsfeld et al., 2006; Ubuka et al., 2012a) as well as in the periventricular nucleus (PeV) and in the region between dorsomedial (DMH) and ventromedial (VMH) hypothalamic nuclei in rat brain (Hinuma et al., 2000; Legagneux et al., 2009). RFRP-expressing neuronal cell bodies mainly locate within the intermediate periventricular nucleus $(\mathrm{PeV})$ of the hypothalamus in macaque (Ubuka et al., 2009a) and in DMH and PVN in sheep (Clarke et al., 2008). On the other hand, immunohistochemistry 
pointed to very high levels of RFRP-1 in the thalamus and midbrain with cell bodies more precisely localized within PeV and VMH nuclei (Fukusumi et al., 2001). Using a rabbit antibody raised against white-crowned sparrow GnIH, immunoreactive cells were found to concentrate within the DMH in rats, mice and Syrian hamsters (Kriegsfeld et al., 2006). In the rat, Syrian hamster and sheep brain, many RFRP-3 fibers establish close contacts with GnRH neurons in the preoptic area (Kriegsfeld et al., 2006; Johnson et al., 2007; Smith et al., 2008a), suggesting a role for this RFamide peptide in the control of reproductive axis. This peptide may also act upstream of GnRH neurons, as RFRP-ir fibers were found to project onto kisspeptin neurons that express NPFF1R in the rat (Rizwan et al., 2012).

NPFF1R mRNA expression was reported in the suprachiasmatic nucleus (SCN), PeV, supraoptic nucleus (SON), and pars tuberalis (PT) in the sheep (Dardente et al., 2008). In rats, NPFF1R mRNA was found in the medial preoptic nucleus, the anteroventral periventricular nucleus (AVPV), medial $(\mathrm{MH})$, paraventricular and periventricular hypothalamic nuclei and in the SCN (Hinuma et al., 2000; Liu et al., 2001). In mice, NPFF1R is weakly expressed in GnRH neurons (Poling et al., 2012). In vitro binding autoradiography in rats showed NPFF1R expression in several regions of hypothalamus, including the anterior (AHA) and lateral (LHA) hypothalamic area and the ARC, VMH, PVN and PeV nuclei (Gouarderes et al., 2004a). In mice, binding autoradiography displayed NPFF1R expression in AHA, ARC, LHA, MH, PVN and PeV (Gouarderes et al., 2004a).

\subsubsection{The RFRPS/NPFFIR system in the regulation of reproduction}

Since the discovery of the inhibitory effect of GnIH in the Japanese quail (Tsutsui et al., 2000), RFRPs (the GnIH homologs in Mammals) have been proposed to counterbalance the positive regulation exerted by kisspeptin on GnRH secretion. Such a hypothesis was supported by the observation of their inhibitory effects both in vitro and in vivo, at the hypothalamic and pituitary levels, in various vertebrate classes including Mammals. At the same time, Hinuma and collaborators provided the first demonstration for a pituitary role of a ñmammalian GnIHò in rats by reporting a dose-dependent effect of RFRP-1 (i.c.v. injection) on plasma prolactin levels. However, no effect of this peptide was observed on LH and FSH levels (Hinuma et al., 2000). More recently, several studies showed that the central and peripheral administration of RFRP-3 was able to decrease the release of gonadotropins, notably LH, in various vertebrates including Mammals (Kriegsfeld et al., 2006; Johnson et al., 2007; Clarke et al., 2008; Kadokawa et al., 2009; Sari et al., 2009; Pineda et al., 2010a).

$\underline{\text { RFRPs/NPFF1R effects on GnRH and gonadotropin secretion }}$

In Mammals, a majority of in vitro and in vivo studies reported an inhibitory effect of RFRPson gonadotrophin secretion, both at hypothalamic and pituitary levels. However, it is important to 
mention here that most experiments in the field that took RF9 (see Fig. 1 and Section 6.2. for further details) as a highly selective NPFF1R antagonist probably led to inaccurate interpretation. Indeed, the rise in gonadotropin release consecutive to RF9 administration, first attributed to a decrease in the endogenous RFRP-3 tone due to NPFF1R blockade (Pineda et al., 2010a, b; Caraty et al., 2012a; Garcia-Galiano et al., 2012; Glanowska et al., 2014), is now clearly assigned to the ability of this compound to activate Kiss1 receptors. Recent studies indeed showed that RF9 promotes intracellular calcium mobilization, inositol phosphate accumulation and ERK phosphorylation in Kiss1R-expressing heterologous cell lines (Kim et al., 2015; Min et al., 2015). Moreover, in NPFF1R KO mice, RF9 $(5 \mathrm{nmol} / 5 \mu \mathrm{L}$; i.c.v. administration) triggers a robust $\mathrm{LH}$ release, similar to that obtained in wild-type littermates, whereas it displays a very weak effect in Kiss1R KO mice (Min et al., 2015). Therefore, owing to its clear Kiss1R agonist component, we will not keep describing RF9 as a tool to modulate LH release via inhibition of NPFF1 receptors.

In vitro, RFRP-3 dose-dependently inhibited the release of LH and FSH induced by GnRH in cell cultures from sheep pituitary and this effect, which was more prominent on LH than on FSH release, strictly depended on the presence of GnRH (Clarke et al., 2008; Sari et al., 2009). RFRP-3 reduced, in a dose-dependent manner, the mobilization of intracellular calcium evoked by $\mathrm{GnRH}$ in ovine pituitary cells in vitro (Clarke et al., 2008). In anterior pituitary cells of cattle, RFRP-3 (0.1 $\mathrm{nM}$ to $1 \mu \mathrm{M}$ concentration range) efficiently decreased $\mathrm{LH}$ levels in the presence of $1 \mathrm{nM} \mathrm{GnRH}$ (Kadokawa et al., 2009). In LßT2 cells, a mouse gonadotrope cell line, RFRP-1 and RFRP-3 also inhibited GnRH-induced LH release (Son et al., 2012). In ram pituitary cell cultures, RFRP-3 totally blocked the increase in expression of LH $\beta$ and FSH $\beta$ mRNA subunits and the stimulation of ERK1/2 phosphorylation induced by GnRH (Sari et al., 2009; Son et al., 2012). Both RFRP-1 and RFRP-3 inhibited GnRH-induced cAMP/PKA activation in L 3 T2 cells (Son et al., 2012). In contrast with former findings, RFRP-3 treatment of isolated anterior pituitary cells from rats, for $3 \mathrm{~h}$ at doses ranging from 1 to $100 \mathrm{nM}$, did not induce any significant variation in $\mathrm{LH}$ levels, in the presence or absence of GnRH (Anderson et al., 2009). The study of RFRP-3 effects on mouse GnRH neurons evidenced three types of responses : $41 \%$ of cells underwent a suppression of firing rate, $12 \%$ displayed an increased firing rate, while no effect was seen in remaining cells (Ducret et al., 2009). The authors also reported that the impact of RFRP-3 on GnRH neurons firing did not significantly vary during the estrous cycle, nor with sex or localization of GnRH neurons.

In vivo, the central injection of RFRP-3 was found to only slightly inhibit gonadotropin release in female and male rats (Johnson et al., 2007; Pineda et al., 2010a) and to reduce GnRH levels in the pig only at a single effective dose of $0.1 \mathrm{nM}$ ( $\mathrm{Li}$ et al., 2013). Intravenous (i.v.) administration of RFRP-3 diminishes the response of ovariectomized (OVX) rats to GnRH (Rizwan et al., 2009). LH pulses (but not FSH) are suppressed in ewes following i.v. RFRP-3 injection (Clarke et al., 2008). 
Although RFRP-3 secreted from the median eminence into the hypophysial portal blood inhibited GnRH-induced LH release from sheep pituitary, no correlation between RFRP-3 and LH pulses could be evidenced (Smith et al., 2012). I.c.v. and i.p. RFRP injections (500 and 600 ng, respectively) into the Syrian hamster caused a drastic reduction (more than $70 \%$ ) in plasma $\mathrm{LH}$ levels within 30 minutes (Kriegsfeld et al., 2006). Significant differences in plasmatic LH and FSH levels were also detected in rats following i.c.v. injection of RFRP-3 (Johnson et al., 2007). More recently, the generation of the first mouse line with constitutive inactivation of the NPFF1 receptor allowed to observe an increase in KiSS-1 expression in the ARC, elevated serum FSH levels and a greater LH response to GnRH, confirming the inhibitory effect of RFRP-3 on reproductive axis (Leon et al., 2014). RFRP-3 (i.c.v.) also impacted sex behaviour in male rats (mounts, intromission, ejaculations) during the photophase (Johnson et al., 2007), in sharp contrast with its lack of effect in mice, sheep and non-human primates (Clarke et al., 2012).

The site of action of RFRPs in Mammals remains controversial. For example, i.v. administration of RFRP-3 was found to promote a significant decrease in serum LH serum levels after 120 minutes while an i.c.v. injection of RFRP-3 had no effect on pulsatile LH secretion in OVX rats (Murakami et al., 2008). Moreover, and in contrast with the well-reported paradigm for an inhibitory effect of RFRP-3 on reproduction, RFRP-3 (i.c.v.) clearly stimulated the gonadotrophic axis via GnRH activation in male Syrian hamsters (Ancel et al., 2012) and short-day Siberian hamsters (Ubuka et al., 2012a), although an inhibitory effect has been shown in female hamsters (Kriegsfeld et al., 2006). Moreover, in the striped hamster, hypothalamic RFRPs mRNA levels were found to be higher in males during the breeding season (Zhao et al., 2014).

The RFRP system and the positive feedback of estrogens on reproductive axis.

In rodents, many studies indicate that inhibition of the RFRP system is necessary for estrogens to exert a positive feedback at hypothalamic and pituitary levels, in agreement with the observation of RFRP fibers in close contact with GnRH neurons in the hypothalamus of rats, Syrian hamsters and sheeps (Kriegsfeld et al., 2006; Johnson et al., 2007; Smith et al., 2008a). RFRP-3 had a central inhibitory effect on GnRH neuronal activity during the GnRH/LH surge induced by estradiol, but not on tonic pulsatile LH secretion in rat (Anderson et al., 2009; Rizwan et al., 2009). RFRP-3 infusion within the AVPV - a region known to mediate the positive feedback of estrogen responsible for the preovulatory surge in rats - suppressed c-Fos cellular activation but only slightly decreased LH concentration at the surge preovulatory peak (Anderson et al., 2009). The targeting of RFRP-containing cells by gonadal steroids in the DMH is supported by the presence of the estrogen receptor $\alpha(\mathrm{ER} \alpha)$ on RFRP-ir cells and by an increase in their c-Fos labelling in response to estrogen (E2) administration (Kriegsfeld et al., 2006). In OVX mice, E2 down-regulated preproRFRP mRNA in the hypothalamus, suggesting that the attenuation of the RFRP endogenous tone by 
estrogen may allow the positive feedback responsible for preovulatory LH surge (Molnar et al., 2011). However, no effects of i.c.v. RFRP-3 injection, at either dose, have been observed on LH pulse frequency or amplitude nor on mean plasma LH concentration in rats (Anderson et al., 2009). $\underline{\text { RFRPs and gonadotropins during the estrus cycle. }}$

The different effects of RFRP-3 during the estrus phase in mice and hamster are modulated by estrogen levels (Kriegsfeld et al., 2006; Gibson et al., 2008; Molnar et al., 2011). The female cycle divides into four stages (metaestrus, diestrus, proestrus and estrus), with different estrogen levels and variable cellular ratios (leucocytes, epithelial cells and cornified cells) within the vagina (Banks, 1993; Priedkalns \& Leiser, 1998). During the estrus cycle, two patterns of GnRH and LH secretion are observed (Fox \& Smith, 1985). The ARC drives a tonic pulsatile secretion of LH that is responsible for the negative feedback, which lasts during almost the entire cycle. The positive feedback is triggered by high concentrations of estrogens that stimulate GnRH release following AVPV activation. This mechanism only occurs at the proestrus stage to provoke the preovulatory LH surge. In the Syrian hamster, SCN fibers contact RFRP-containing neuronal cells. Moreover, during proestrus, the number and activity of RFRP-containing cells are reduced in the DMH compared to diestrus, where levels are maximal (Gibson et al., 2008). During the LH surge, early gene expression is reduced in RFRP-positive cells, under the dependence of estrogens (Gibson et al., 2008). As well, c-Fos-positive RFRP-1-ir neurons are significantly higher during diestrus than in proestrus or estrus stages (Jorgensen et al., 2014). RFRP-3 suppresses cellular activation within the AVPV, a region known to robustly express c-Fos, especially in kisspeptin-ir neurons during the preovulatory surge (Smith et al., 2006a). However, no effect of estrogens on RFRPs mRNA levels has been observed in female rats (Quennell et al., 2010).

The RFRP system and the regulation of sexual organ activity

Central and direct actions of RFRPs on testicular activity have been extensively reviewed by Ubuka and coworkers (Ubuka et al., 2014). RFRPs and NPFF1R mRNAs are expressed in rodent (Zhao et al., 2010; Singh et al., 2011a, b), pig (Li et al., 2012) and human (Oishi et al., 2012) gonads, in agreement with the potential autocrine/paracrine control of testicular activity exerted by GnRH and RFRPs (Anjum et al., 2012). RFRPs and NPFF1R protein expression is observed in the testis of Syrian hamsters, and more particularly in myoid cells of all tubules and spermatocytes, round spermatids and elongated spermatids, with various patterns according to the phase of the seminiferous epithelial cycle (Zhao et al., 2010). ISH studies in Syrian hamster testis showed a strong expression of RFRPs and NPFF1R mRNAs in spermatocytes and spermatids (Zhao et al., 2010). A significant correlation between RFRPs, GnRH and GnRH-R immunostaining levels was observed in mice testis from birth to senescence (Anjum et al., 2014). RFRP-ir levels decrease from birth to pre-puberty, whereas they significantly increase during pubertal and reproductive stages to 
senescence. Compared to control mice, RFRP treatment induced a significant and dose-dependent decrease in GnRH-R expression in the testis (Anjum et al., 2012) and the inhibition of testicular activities in adult mice, either directly or through GnRH (Anjum et al., 2014). RFRP-3 was also found to decrease the proliferation of germinal cells, to increase apoptotic markers and to inhibit $\mathrm{LH}$ receptor expression, 3 $\beta$-Hydroxysteroid deshydrogenase and P450 side-chain cleavage (enzymes involved in sexual steroid synthesis) and testosterone secretion from mice testes (Anjum et al., 2014).

\subsection{The NPFF/NPFF2R system and the regulation of reproductive axis in Mammals}

\subsubsection{Localization of the NPFF/NPFF2R system in reproduction pathways}

In rat and mouse brain (Figure 8), NPFF-immunoreactive neurons, fibers and terminals mainly locate in the hypothalamus (Kivipelto et al., 1989). They massively project onto the neurohypophysis and establish synaptic contacts with pituitary cells (Kivipelto et al., 1989; Boersma et al., 1993). At the periphery, the NPFF2R protein was found to be expressed in late elongated spermatids of Syrian hamsters testis (Zhao et al., 2010).

\subsubsection{Function of the NPFF/NPFF2R system in reproduction}

To our knowledge, no evidence for a function of NPFF in reproduction has been published to date. As reported by Pineda and collaborators, central injection of NPFF in rats had no effect on circulating LH and FSH levels (Pineda et al., 2010a).

\subsection{The PrRPS/ PrRP-R system and the regulation of reproductive axis in Mammals}

\subsubsection{Localization of PrRPs and PrRP-R in reproduction pathways}

A schematic presentation of the localization of these peptides and their receptor in rodent brain areas involved in reproduction is provided in Figure 8.

In the rat, PrRP mRNA is distributed throughout the CNS, with highest levels in the DMH and medulla oblongata and moderate expression in the hypothalamus (Hinuma et al., 1998). However, bioactive PrRPs have been found to be significantly enriched within the hypothalamus as compared to the medulla oblongata in rat brain (Fujii et al., 1999). Within the medulla oblongata, PrRPs are expressed in the ventrolateral reticular formation (VLRN), the commissural nucleus of the lateral NTS and the dorsal medullary of the reticular field where noradrenergic neurons are located (Chen et al., 1999). These noradrenergic neurons project onto the hypothalamus (Chen et al., 1999) where they stimulate GnRH neurons in the medial septum diagonal band and in the POA (Wright \& 
Jennes, 1993). In the hypothalamus, PrRPs were mainly located in the bed nucleus of striaterminalis (BST; Matsumoto et al., 1999), in close proximity to the POA area where PrRP-R-ir and GnRH-ir neurons were shown to colocalize (Feng et al., 2007). Both PrRP and PrRP-R mRNA were present in rat testis and epididymis (Nieminen et al., 2000).

Expression of PrRPs in female rat brain varies during the estrus cycle and is linked to GnRH expression (Feng et al., 2007). Sex steroids, which are involved in the phase transition during the estrus cycle, have a direct influence on PrRP mRNA and peptide expression in rats (Kataoka et al., 2001). In ovariectomized rats, estrogen up-regulated PrRP mRNA levels in the NTS and VLRN while progesterone induced a significant increase in PrRP mRNA only in the NTS. In the latter region, double-label immunocytochemistry allowed to identify a majority (70\%) of PrRP immunoreactive neurons that co-expressed ER $\alpha$ receptors (Kataoka et al., 2001).

PrRP-R mRNA was found in several hypothalamic regions such as the medial preoptic area and the periventricular, paraventricular and dorsomedial nuclei in rats (Roland et al., 1999) and was also detected in human and rat pituitaries (Welch et al., 1995; Hinuma et al., 1998; Fujii et al., 1999).

\subsubsection{Function of the PrRPs/PrRP-R system in the reproductive axis}

There is some evidence for an action of PrRPs on the reproductive axis and gonadotropin secretion. Although PrRP-31 failed in promoting LH, FSH or PRL release from rat anterior pituitary cells in vitro (Kawamata et al., 2000), i.c.v. injection of PrRP-31 in rats led to a significant increase in plasma LH, FSH and testosterone levels, and stimulated the release of GnRH from male rats hypothalamic explants (Seal et al., 2000). Administration of anti-PrRP serum significantly reduced the amplitude of the LH surge in female rats, suggesting a role of PrRPs in the preovulatory signal (Hizume et al., 2000). The secretory profile of PrRPs in the rat hypothalamus, before and during LH surge, has been examined using push-pull perfusion of sex steroids (Watanobe, 2001). An early rise in PrRPs and a subsequent release of GnRH, followed by the LH surge, have been observed in the medial preoptic area (POA), a region enriched in GnRH neurons. Thus, PrRPs may mediate the steroid-induced LH surge through the activation of GnRH neurons in POA (Watanobe, 2001).

\subsection{The Kisspeptins/Kiss1R system in the regulation of reproductive axis}

The pivotal role of Kisspeptins in reproduction was first apprehended with the isolation of Kiss1R mutations in idiopathic hypogonadotropic hypogonadism (de Roux et al., 2003; Seminara et al., 2003) and the observation of a similar phenotype in KiSS-1 KO mice (d'Anglemont de Tassigny et al., 2007; Lapatto et al., 2007). Kiss1R null mice were also reported to be infertile (Seminara et 
al., 2003). Altogether, these data demonstrated that the Kisspeptins/Kiss1R system is mandatory for the development of puberty and the control of reproduction. As a consequence, over the past decade, research on the involvement of this system in the control of reproduction of many different species has been very active and led to the publication of a considerable amount of literature.

In this review, we have tried to summarize the main findings in the field. Further details are available in recent reviews that specifically address this topic (Pinilla et al., 2012; Wahab et al., 2013; Clarke et al., 2015; Plant 2015).

\subsubsection{Localization of Kisspeptins and Kiss $1 R$ in reproductive pathways and organs}

The distribution of kisspeptins in the mammalian nervous system has been detailed in recent reviews (Mikkelsen \& Simonneaux, 2009; Lehman et al., 2010). A schematic presentation of the localization of these peptides and their receptor within the rodent brain is provided in Figure 8 .

In the mammalian brain, two main populations of kisspeptinergic neurons are known to be involved in reproduction regulation and feedback signals. The presence of KiSS-1 mRNA and protein in the arcuate (ARC) and dorsomedial (DMH) hypothalamic nuclei and the preoptic region, including the preoptic area (POA) and the anteroventral periventricular nucleus (AVPV), were identified by in situ hybridization (Gottsch et al., 2004; Irwig et al., 2004; Han et al., 2005; Shahab et al., 2005; Smith et al., 2005a, 2006b, 2007, 2008b, 2009a, 2010; Estrada et al., 2006; Revel et al., 2006; Adachi et al., 2007; Kauffman et al., 2007a, b; Rometo et al., 2007; Takase et al., 2009; Ansel et al., 2010) or immunohistochemistry (Kinoshita et al., 2005; Clarkson \& Herbison, 2006; Franceschini et al., 2006; Goodman et al., 2007; Greives et al., 2007; Mason et al., 2007; Decourt et al., 2008; Ramaswamy et al., 2008, 2010; Clarkson et al., 2009; Ohkura et al., 2009; Smith et al., 2009a; Takase et al., 2009; Cheng et al., 2010; Desroziers et al., 2010; Hrabovszky et al., 2010; Wakabayashi et al., 2010). However, within these main regions, each mammalian species displays a specific KiSS-1 distribution pattern.

KiSS-1 expression displays a marked sexual dimorphism in almost all mammalian species studied to date (Ansel et al., 2010; Cheng et al., 2010; Hrabovszky et al., 2010). In rodents, females express a significantly higher number of Kiss1R/Kiss-ir neurons than males in AVPV, in direct link with the absence of testosterone during a perinatal critical window (Gogan et al., 1980, 1981; Corbier, 1985; Connolly \& Resko, 1994; Simerly, 1998, 2002; Smith et al., 2005a, b; Clarkson \& Herbison, 2006; Adachi et al., 2007; Kauffman et al., 2007a; Ansel et al., 2010). This suggest that in adults, sex differences in the number of Kiss1R/Kiss-ir neurons in AVPV is not due to difference in circulating levels of gonadal steroids. In contrast, no sex differences were found in the ARC (Clarkson \& Herbison, 2006; Adachi et al., 2007; Kauffman et al., 2007a). Kisspeptin immunoreactive fibers contact GnRH neuron cell bodies (Kinoshita et al., 2005; Clarkson \& 
Herbison, 2006; Smith et al., 2008a) and their terminals in the median eminence (Smith et al., 2011). Kisspeptin neurons in the ARC, POA and AVPV regions of sheep, rat and mouse brain coexpress steroid receptors (Lehman et al., 2010).

In all mammalian species, high levels of Kiss1 receptor mRNA and protein are found in GnRH neurons of the hypothalamus and in cells of the periventricular $(\mathrm{PeV})$ region of the hypothalamus, suggesting that the effects of kisspeptins are mediated by these neurons (Irwig et al., 2004; Han et al., 2005; Colledge, 2009; Smith et al., 2009a; Herbison et al., 2010). In rat brain, Kiss1R mRNA was detected in ARC, DMH, LHA, POA and AHA hypothalamic regions (Lee et al., 1999). In the periphery, KiSS-1 and Kiss1R have been detected in rat ovary (Terao et al., 2004; Castellano et al., 2006b) and oviduct (Gaytan et al., 2007), as well as in human and marmoset ovaries (Gaytan et al., 2009). Although the functional role of this system in male gonads remains controversial, expression of KiSS-1 and Kiss1R has been reported in testis (Mei et al., 2013; Salehi et al., 2015).

\subsubsection{Functions of the Kisspeptins/Kiss1R system in reproduction}

The involvement of kisspeptin in different aspects of reproduction in Mammals has been recently reviewed (Pinilla et al., 2012; Wahab et al., 2013; Clarke et al., 2015; Plant 2015). This peptide is involved in the stimulation of gonadotropin release, in the steroid feedback loop of the gonadal axis and in the pubertal switch at hypothalamic, pituitary and gonadal levels. Thus, the disruption of the KiSS-1/Kiss1R system is responsible for severe reproductive disorders.

Kisspeptins stimulate the secretion of gonadotropins

Kisspeptins induce a massive release of gonadotropins via its stimulatory action on GnRH neurons (Han et al., 2005). Kisspeptin-induced LH secretion is blocked by the GnRH antagonist, acyline (Gottsch et al., 2004; Irwig et al., 2004). In vitro, kisspeptin-10-mediated GnRH secretion from hypothalamic fragments is absent in Kiss1R null mice (d'Anglemont de Tassigny et al., 2008). Systemic or i.c.v. administration of kisspeptin-10 or -54 stimulates LH release in a large variety of mammalian species, including rodents (Gottsch et al., 2004; Irwig et al., 2004; Matsui et al., 2004; Kinoshita et al., 2005; Messager et al., 2005; Navarro et al., 2005; Patterson et al., 2006; Thompson et al., 2006; Tovar et al., 2006; Greives et al., 2007), sheep (Messager et al., 2005), cows (Kadokawa et al., 2008) and primates (Dhillo et al., 2005, 2007; Shahab et al., 2005; Plant et al., 2006). Similar effects of kisspeptins on FSH secretion have been described in rodents (Gottsch et al., 2004; Matsui et al., 2004; Navarro et al., 2005; Thompson et al., 2006; Roa et al., 2008), sheep (Caraty et al., 2007) and humans (Dhillo et al., 2005), with noticeable differences in the delay and sensitivity of the FSH response as compared to the LH one (Navarro et al., 2005). On cultured baboon pituitary cells, kisspeptin evoked a dose-dependent release of LH but not of FSH (Luque et al., 2011). Finally, there is also emerging evidence to consider kisspeptin as a good candidate for 
therapeutic outcomes in the field of human reproduction (Clarke et al., 2015). Indeed, kisspeptin increases LH pulsatility in healthy volunteers as well as in patients with defects in gonadotropin secretion (George et al., 2011; Chan et al., 2011; Jayasena et al., 2013; Jayasena et al., 2014). In addition, kisspeptin is able to trigger oocyte maturation and embryo implantation during in vitro fertilization, without side-effects encountered with standard molecules (Jayasena et al., 2014; Abbara et al., 2015).

Kisspeptins are involved in the steroidal feedback loop

The pulsatile release of GnRH into the hypophyseal portal blood stimulates the synthesis and secretion of gonadotropins in the peripheral circulation, allowing LH and FSH to reach the gonads and to stimulate the secretion of sex steroids. Subsequently, sex steroids initiate either positive or negative feedback loops, depending on tissues or brain regions that are targeted and on the presence of the kisspeptin system (Navarro et al., 2004; Smith et al., 2005a, b). For example, the anteroventral periventricular nucleus of the hypothalamus (AVPV), which is responsible for the stimulation of GnRH secretion and subsequent preovulatory LH surge and rise in FSH level, is submitted to an estrogen-positive feedback (Wiegand \& Terasawa, 1982; Adachi et al., 2007). In rodents, the decrease in kisspeptin mRNA expression and immunoreactivity observed in the AVPV following gonadectomy can be restored by estrogens (Smith et al., 2005a; Adachi et al., 2007). Estrogens up-regulate KiSS-1 gene expression in AVPV through activation of estrogen ER $\alpha$ receptors (Adachi et al., 2007). Kisspeptin neurons in the AVPV mediate the positive feedback of estrogen by opposing the inhibitory action of GABA signalling in GnRH neurons (Zhang et al., 2009). The arcuate nucleus (ARC) can also mediate a positive feedback, at least in the sheep (Estrada et al., 2006). On the other hand, in rodents, sheep and primates, the negative feedback exerted by sex steroids is controlled at the ARC (infundibular nucleus in humans) level. There, estrogens suppress the frequency of GnRH pulses and reduce kisspeptin mRNA expression ( Smith et al., 2005a; Adachi et al., 2007; Li et al., 2009). In addition, and in marked contrast with former observations in the AVPV, high levels of estrogens counteract the increase in kisspeptin mRNA and immunoreactivity induced by gonadectomy in rodents (Smith et al., 2005a; Adachi et al., 2007).

\section{Kisspeptins as a switch for puberty}

Kisspeptins are crucial for puberty onset. During the pubertal transition, an increase in hypothalamic kisspeptin mRNA expression was found to occur in rats (Navarro et al., 2004), mice (Han et al., 2005) and monkeys (Shahab et al., 2005). The release of kisspeptin-54 in the median eminence of female rhesus monkeys coincided with the pubertal increase in GnRH release (Keen et al., 2008). Kiss1R and KiSS-1 KO mice of both genders display striking reproductive abnormalities, such as underdevelopment of gonads and accessory reproductive organs, reduced levels of gonadotropins and steroid hormones, absence of estrous cycle or impaired gametogenesis 
and sexual maturation (Funes et al., 2003; Seminara et al., 2003; d'Anglemont de Tassigny et al., 2007; Dungan et al., 2007; Kauffman et al., 2007b; Lapatto et al., 2007). In humans, loss-offunction mutations in Kiss1R cause hypogonadotropic hypogonadism (de Roux et al., 2003; Seminara et al., 2003; Lanfranco et al., 2005; Semple et al., 2005) whereas gain-of-function mutations cause precocious puberty (Teles et al., 2008).

Kisspeptins as a regulator of sexual organs activity

In vitro, kisspeptins were found to favor oocyte maturation (Saadeldin et al., 2012) and to induce the production of progesterone in cultured rat luteal cells (Peng et al., 2013). Kisspeptins are expressed in Leydig cells in the testes, in the cumulus oocyte complex and in the epithelium of ovarian and oviductal tissues. Kiss1R is found in seminiferous tubules and more particularly in the acrosomal region of spermatids and mature spermatozoa (Hsu et al., 2014). Morover, kisspeptin-10 challenge of Fluo-4 loaded sperm was found to promote an increase in intracellular calcium indicating that these highly specialized cells express a functional Kiss1R in these highly specialized cells, which suggests a possible direct role of this receptor in mammalian fertilization (Hsu et al., 2014).

\subsection{The $Q R F P S / Q R F P-R$ system and the regulation of reproductive axis in Mammals}

\subsubsection{Localization of the $Q R F P S / Q R F P-R$ system in reproduction pathways}

A schematic presentation of the localization of QRFPs and QRFP-R1/R2 in rodent brain areas involved in reproduction is provided in Figure 8.

Prepro-QRFP mRNA was originally shown to be mainly expressed in discrete rat brain areas such as the ventromedial hypothalamic nucleus (VMH) and the lateral hypothalamic (LHA) area (Chartrel et al., 2003). Other studies evidenced also QRFP mRNA in ARC of rats (Fukusumi et al., 2003; Kampe et al., 2006) and PeV of mice (Takayasu et al., 2006). In adult female rat pituitary, maximal relatives value of QRFP-R and QRFP transcripts were found during diestrus, while a lower expression was seen during the period preceding full pubertal development (Navarro et al., 2006).

In rodent brain, the distribution of QRFP- receptors markedly depends on the receptor isoform and the animal species which are considered. Within mouse brain areas involved in reproduction, QRFP-R1 mRNA was observed in the VMH while QRFP-R2 mRNA was detected in several hypothalamic regions such as AVPV, PVN and LHA and in the SCN (Takayasu et al., 2006). In rats, both QRFP-R1 and QRFP-R2 isoforms were found in the preoptic (POA) and anterior (AHA) hypothalamic areas, which contain the main populations of GnRH neurons, in the PVN and VMH nuclei as well as in the bed nucleus of stria terminalis (BST). In contrast with mice, rat QRFP-R1 
mRNA preferentially located within the ARC and LHA (Fukusumi et al., 2003; Kampe et al., 2006). QRFP and QRFP-R mRNA were also found in the human pituitary (Jiang et al., 2003).

In peripheral tissues, QRFP and QRFP-R mRNAs were found in humans (Jiang et al., 2003; Baribault et al., 2006) and rodents (Fukusumi et al., 2003; Jiang et al., 2003; Takayasu et al., 2006) testes. In mice testis, the QRFP-R2 isoform appeared to predominate (Takayasu et al., 2006).

\subsubsection{Function of the $Q R F P S / Q R F P-R$ system in the reproductive axis}

Known as a hypophysiotropic peptide (Chartrel et al., 2003), QRFP-26 emerged in the recent years as a new actor in the regulation of the reproductive axis (Navarro et al., 2006; Patel et al., 2008). Indeed, following its incubation with pituitary tissue from adult female rats at diestrus, QRFP-26 was found to induce the release of LH in a dose-dependent manner $\left(10^{-10}\right.$ to $\left.10^{-6} \mathrm{M}\right)$ and of FSH at micromolar concentrations. Moreover, the application of high doses of QRFP-26 on this tissue enhanced the GnRH-evoked secretion of LH and FSH, without any significant effect on PRL release (Navarro et al., 2006). The i.c.v. injection of QRFP-26 led to a significant increase in serum LH (but not FSH) level, in high and low estrogen environment, as well as during the afternoon preceding preovulatory surge in female rats. A systemic administration of QRFP-26 was also able to elicit a significant increase of LH (but not FSH) in cyclic female rats at diestrus. No effect has been observed in males, suggesting a sexual dimorphism in terms of gonadotropin activation by QRFP-26 (Navarro et al., 2006). However, in male rats, the i.c.v. administration of QRFP-43 promoted a significant increase in LH and FSH plasma levels, an effect which was blocked by a GnRH antagonist (Patel et al., 2008). The central action of QRFP-43 was also confirmed on hypothalamic explants from male rats, with a significant increase in GnRH release while no changes in LH or FSH levels were found in the anterior pituitary (Patel et al., 2008). No effect on circulating gonadotropin or sex steroid levels was observed after intraperitoneal administration of high doses of this peptide (Patel et al., 2008). Similarly, in the adult male rhesus monkey, peripheral administration QRFP-26 and QRFP-43 had no effect on testosterone and prolactin secretion (Wahab et al., 2012).

\subsection{RF-amide peptides and control of reproduction in seasonal breeders}

To ensure optimal conditions for their reproduction in temperate climates, some animals, called ñseasonal breedersò, are sensitive to environmental changes and undergo a photoperiodic control of their reproductive activity. Photoperiod is an important physical factor that modifies sexual behaviour and molecular activity in the brain. In the wild world, offspring are born during the most favourable season, usually spring. However, according to gestation duration, photoperiodic animal 
species may breed at different periods of the year, thereby defining đóng-dayô(LD) and óhort-dayô (SD) breeders. Given their short gestation, small animals (like the hamster) reproduce in springtime, when days are long. On the other hand, animals with a longer gestation period (about 4 months for the sheep), are called short-day breeders, or fall-breeders.

Time of breeding is directly related to gestation length and critically depends on environmental signals, among which the duration of light exposure (called photoperiod) is the main protagonist. Indeed, photoperiod is a reliable indicator of seasonal changes, with day length being translated at the brain level with melatonin, a neurohormone synthetized and secreted by the pineal gland during the night, and inhibited by light. As a consequence, short days are associated with long nocturnal melatonin secretion, whereas long days correspond to short nocturnal melatonin secretion. Depending on the breeding animal, via a process that is not well understood, translation of the melatonin signal makes sheep sexually active in short-day (SD) and hamster sexually active in longday (LD). The distribution of melatonin binding sites in the brain differs across species (MassonPevet et al., 1994), although a high density of melatonin receptors has been observed in the pars tuberalis of the adenohypophysis in many mammalian species (Masson-Pevet \& Gauer, 1994). This region emerged as an important structure involved in melatonin effects on seasonal reproductive function (Klosen et al., 2002; Dardente et al., 2003; Johnston et al., 2006). Owing to their regulation by melatonin, much interest has been recently devoted to kisspeptin and RFRP-3 peptides, now regarded as major actors of the seasonal control of reproduction in Mammals (Simonneaux et al., 2013).

\subsubsection{The Kisspeptin system and the seasonal control of reproduction}

KiSS-1 appears to be the missing link between HPG and melatonin. In seasonal animals such as long-day (LD) and short-day (SD) breeders, KiSS-1 gene expression is regulated by the photoperiod.

In the Syrian hamster (a long-day breeder), kisspeptin-10 challenge counteracts the suppressive effect of short-light exposure on reproduction and restores testicular activity, indicating that low kisspeptin levels signal an arrest of reproduction in short-days (Revel et al., 2006). Melatonin and sex steroids effects on kisspeptin neurons seem to play a crucial role in timing the neuroendocrine control of the gonadotropic axis in the Syrian hamster. Indeed, in SD compared to LD, KiSS-1 expression in the ARC and the AVPV of male and female hamsters is strongly reduced, as are circulating levels of testosterone in agreement with the stimulatory role of kisspeptins on the gonadotropic axis (Revel et al., 2006; Simonneaux et al., 2009; Ansel et al., 2010; Bartzen-Sprauer et al., 2014). Moreover, the increase in KiSS-1 mRNAs in the ARC observed after castration of LD Syrian hamsters (Revel et al., 2006; Ansel et al., 2010) is consistent with the inhibitory effect of sex 
steroids on KiSS-1 expression in this region. Thus, the decrease in KiSS-1 expression observed in short days in the ARC is not due to a diminution of testosterone levels, whereas in the AVPV, it likely results from the lowering of circulating testosterone (Ansel et al., 2010). On the other hand, pinealectomy prevents the down-regulation of KiSS-1 expression in SD Syrian hamsters (Revel et al, 2006) and increases the number of KiSS-1 neurons in the ARC, but not in the AVPV, of male or female SD hamsters (Ansel et al., 2010). Finally, some Kiss-1-positive cells were found in the mediobasal hypothalamus where melatonin target sites locate, providing additional support for a link between melatonin and kisspeptin systems (Goldman et al., 2001; Hastings et al., 1988; Maywood et al., 1995; Lewis et al., 2002; Mikkelsen \& Simonneaux, 2009). Thus, in the Syrian hamster, the long nocturnal peak of melatonin in SD seems to inactivate the reproductive state via an inhibition of KiSS-1 expression which is either sex steroid-dependent in the AVPV or sex steroid-independent the ARC. However, in the Siberian hamster - which also belongs to long-day breeders- kisspeptin levels in the ARC were found to be higher under SD conditions than under LD ones (Greives et al., 2007; Mason et al., 2007; Simonneaux et al., 2009). Such unexpected findings raised several hypotheses and still merit further clarification (Simonneaux et al., 2013).

As in long-day breeders, kisspeptin expression in sheep (a short-day breeder) varies with the season and sex steroid levels. In ewe, as observed in the Syrian hamster, acute and chronic administration of kisspeptins during anestrus reactivates the gonadal axis and triggers ovulation (Caraty et al., 2007; Smith et al., 2009b). The expression of KiSS-1 mRNA in the ARC and the number of contacts between kisspeptin and GnRH neurons are higher during the breeding season, as compared to the non-breeding period. Such a differential expression is independent from sex-steroid feedback as it is preserved in OVX ewes, with or without estrogen replacement (Franceschini et al., 2006; Caraty et al., 2007; Smith et al., 2008b). Furthermore, estrogens have been found to exert a stronger inhibition of KiSS-1 expression in anestrus rather than during the breeding season, suggesting a change in sensitivity towards negative estrogen feedback according to the season (Smith et al., 2008a).

Altogether, data from Syrian hamster and sheep are consistent with a role for ARC kisspeptin neurons in the control of seasonal reproduction. They also indicate that the same melatonin message can lead to opposite reproductive states in different species, according to the breeding type (Karsch et al., 1984; Revel et al., 2008). In sheep brain, melatonin receptors have been localized in the premammillary hypothalamic area, a structure anatomically closely related to ARC (Malpaux et al., 1998). However, a direct effect of melatonin on kisspeptin neurons has not been demonstrated yet, although the long melatonin peak in SD clearly suppresses sexual activity in hamsters and increases it in sheep. 


\subsubsection{The RFRPS/NPFF1R system and the seasonal control of reproduction}

In Mammals, RFRP-expressing neurons are mainly located in the mediobasal hypothalamus (Ukena \& Tsutsui, 2001; Kriegsfeld et al., 2006; Clarke et al., 2008; Dardente et al., 2008; Revel et al., 2008; Smith et al., 2008a; Rizwan et al., 2009). In this region, RFRPs expression was found to vary according to photoperiod in LD and SD breeders (Dardente et al., 2008; Revel et al., 2008; Smith et al., 2008a; Mason et al., 2010; Ubuka et al., 2012b; Janati et al., 2013), but not in nonphotoperiodic animals, such as rats (Revel et al., 2008). This region was found to contain melatonin binding sites and its electrolytic lesion prevented the inhibitory effect of melatonin on reproduction of the long-days breeder Syrian hamster (Maywood et al., 1996).

In seasonal Mammals, RFRP-immunoreactivity decreases in short-day periods, whatever the LD or SD breeding type of the species considered. In Syrian and Siberian hamsters, RFRPs mRNA levels are higher in the long photoperiod than during the short one (Revel et al., 2008), an observation which is inconsistent with the inhibitory role of RFRPs on reproductive axis previously reported. In other long-day breeders, such as the Jerboa and European hamsters, RFRPs expression is also inhibited in SD-maintained animals (Janati et al., 2013). In SD-adapted Syrian and Siberian hamsters (no reproductive activity), hypothalamic RFRPs mRNA was strongly down-regulated, in association with a decrease in RFRP immunoreactivity in perikarya and fibers (Revel et al., 2008; Mason et al., 2010; Ubuka et al., 2012a). Remarkably, in short-day breeders, RFRPs mRNA and protein expression were also reduced in SD conditions when animals are sexually active (Dardente et al., 2008; Smith et al., 2008a). In sheep, hypothalamic RFRPs mRNA levels are about 20\% higher under LP than SP conditions (Dardente et al., 2008). In the marsupial brain, there is a twofold increase in RFRP-3 cell body number in the hypothalamus during the non-breeding season as compared to the breeding one (Harbid et al., 2013). In agreement with its inhibitory role on the gonadotropic axis, RFRP-3 input on GnRH neurons varied with the season (Clarke et al., 2008; Smith et al., 2008a). The analysis of the sequence of the goat RFRP gene highlighted a positive correlation between gene mutations and average daily sunshine duration, reproductive seasonality and litter size (Huang et al., 2012).

Testosterone treatment of SD-adapted Syrian hamsters (or testis ablation of LD-animals) did not alter RFRP mRNA levels (Revel et al., 2008). However, estrogens down regulates RFRP mRNA levels in OVX mice (Molnar et al., 2011) and ER- $\alpha$ is present in a subset of RFRP-positive neurons in the hypothalamus where they respond to estrogen administration (Kriegsfeld et al., 2006; Gibson et al., 2008; Molnar et al., 2011), suggesting a role for sex steroids in the regulation of RFRPs expression.

Melatonin modulates RFRPs mRNA levels according to the photoperiod (Revel et al., 2008; Ubuka et al., 2012a). For example, during the short-day period, melatonin was found to decrease 
RFRPs mRNA levels in the Siberian hamster (a long-day breeder), whereas the pineal hormone induced the expression of $\mathrm{GnIH}$ in quails which are sexually inactive during this period (Ubuka et al., 2005; Revel et al., 2008; Ubuka et al., 2012a). At the periphery, RFRP and NPFF1R mRNA expression in male Syrian hamster testes is impacted by photoperiod and reproductive conditions (Zhao et al., 2010).

Often referred to as an inhibitor of reproduction in Mammals, RFRPs cannot be reduced to this simple assertion as they exert a sexually and photoperiodically dimorphic action in hamsters (Ancel et al., 2012; Ubuka et al., 2012a). While an inhibitory effect on gonadotropin release was reported in OVX female Syrian hamsters (Kriegsfeld et al., 2006), the i.c.v. administration of RFRP-3 to male Syrian hamsters stimulated GnRH cell activity and increased LH production and circulating levels of testosterone. The i.c.v injection of RFRP-3 in male Syrian hamsters under photo-inhibitory conditions, led to an increase in LH release and reactivated the reproductive axis after 5 weeks of continuous treatment, similarly to what was observed under LD conditions (Ancel et al., 2012), and stimulates gonadotropin secretion in LD Siberian hamster (Ubuka et al., 2012a).

No photoperiod-dependent effects have been reported in seasonal Mammals for the other members of the RF-amide peptide and receptor family. In contrast to kisspeptins, the roles of RFRPs in reproduction seem to be more difficult to analyse owing to their strong variations according to the LD or SD breeding type of the animal models.

Overall, all mammalian RF-amide peptides, except NPFF, have been implicated in the modulation of reproduction. Among them, special attention was paid to Kisspeptin/Kiss1R and RFRPs/NPFF1R systems as main regulators of central control of reproduction, acting on GnRH neurons. These neuropeptides integrate environmental signals as photoperiod and sexual steroids to permit a regulation of the reproductive axis with variable intensity. Nevertheless, although the role of kisspeptins as potent stimulators of the reproductive axis has been well-established, RFRPs function remains controversial in Mammals. They appear to regulate reproduction mainly in seasonal breeders, but in opposite way according to the reproductive state, sex and species. In the future, the identification of novel pharmacological tools, as highly selective NPFF1R antagonists, should be very helpful to clearly decipher the precise action of this system in the wide and complex field of reproduction.

\section{Mammalian RF-amide peptides and their receptors in nociception and pain}

Several reports indicate that mammalian RF-amide peptides and their receptors are involved in the modulation of nociception. Here, we will briefly present their distribution in pain pathways in 
mice, rats and human and summarize current knowledge on their roles in nociception and in the modulation of opiates effects. These topics have been discussed into more details in a recent review (Ayachi \& Simonin, 2014).

\subsection{The RFRPs/NPFF1R system in nociception and pain}

\subsubsection{Localization in pain pathways}

Figure 9 presents the distribution of RFRPs and their NPFF1 receptor in the regions of rat and mouse central and peripheral nervous systems involved in the modulation of nociception.

RFRP-1 and RFRP-3 containing neuronal cell bodies and fibers are widely distributed in the brain, with high levels in the hypothalamus. RFRP-1/-3 immunoreactivity was detected in several regions known to be implicated in the modulation of nociception like the spinal trigeminal nucleus (STN), nucleus tractus solitarius (NTS), dorsal medial raphe nucleus (DMR), locus coeruleus (LC), parabrachial nucleus (PB), periaqueductal gray (PAG), amygdala (Amd), ventral postlateral nucleus (VPL) of the thalamus, and the paraventricular nucleus (PVN) of the hypothalamus (Ukena \& Tsutsui, 2001; Yano et al., 2003). ISH studies also revealed the presence of RFRP mRNA in the lateral hypothalamic area (LHA) of rat brain (Hinuma et al., 2000). In addition, dense networks of RFRP-immunoreactive fibers and high levels of RFRP mRNA were found in the dorsal horn of mouse spinal cord (Ukena \& Tsutsui, 2001; Yang \& Iadarola, 2003). In rats, much lower levels or no RFRP expression at all were observed in this region (Yano et al., 2003; Pertovaara et al., 2005).

In rats or mice, the NPFF1 receptor mRNA locates within the raphe nucleus, locus coeruleus, parabrachial nucleus, periaqueductal gray, amygdala, ventral tegmental area (VTA), habenula (Hab), nucleus accumbens (NAc) and the paraventricular nucleus of the hypothalamus (Bonini et al., 2000; Hinuma et al., 2000; Liu et al., 2001). NPFF1R mRNA expression and binding sites in the spinal cord or in the nucleus tractus solitarius were reported to vary between rat and mouse strains (Bonini et al., 2000; Liu et al., 2001; Gouarderes et al., 2004a). In humans, NPFF1R mRNA was detected in several areas, including in pain- or nociception- associated regions such as the spinal cord, hypothalamus, and amygdala (Bonini et al., 2000).

\subsubsection{Modulation of nociception}

Under basal nociceptive conditions, RFRP-1 effect primarily depends on opioid receptor activation as the peptide had no effect when injected (i.c.v.) alone while it decreased or blocked morphine analgesia (Liu et al., 2001). Conversely, i.c.v. RFRP-3 administration in mice had no effect on morphine analgesia but significantly decreased thermal nociceptive threshold when injected alone (Elhabazi et al., 2013). A few studies examined RFRPs effects under chronic pain 
conditions; in a neuropathic model, RFRP-1 has been shown to induce an anti-nociceptive effect in rats (Pertovaara et al., 2005).

\subsection{The NPFF/NPFF2R receptor system in pain and nociception}

\subsubsection{Localization in pain pathways}

A schematic distribution of NPFF and NPAF peptides and of their NPFF2 receptor in regions of rat and mouse central and peripheral nervous systems involved in nociception and pain modulation is presented in Figure 9.

NPFF is unevenly expressed in the rat CNS with highest levels localized to the superficial layers of the dorsal spinal cord (Yang et al., 2008). NPFF mRNA was found in the spinal trigeminal nucleus, nucleus tractus solitarius and paraventricular hypothalamus nucleus; NPFF-ir fibers were also detected in parabrachial nuclei, amygdala, and nucleus accumbens (Panula et al., 1996; Yang \& Iadarola, 2006; Yang et al., 2008, for reviews). The presence of NPFF in dorsal root ganglia (DRG) remains controversial (Yang \& Iadarola, 2006; Ayachi \& Simonin, 2014). A few studies focused on NPAF which was also found in mouse and rat spinal cord (Bonnard et al., 2001; Yang et al., 2008). In humans, the presence of NPFF in the spinal cord was revealed by using a specific radioimmunoassay (Majane et al., 1988), while NPFF mRNA was detected both in the medulla and spinal cord (Nystedt et al., 2002). NPFF and NPAF peptides were also detected in human cerebrospinal fluid using a radioimmunoassay (Sundblom et al., 1997) and mass-spectrometry (Burlet-Schiltz et al., 2002).

The localization of NPFF2R mRNA and protein in rodents has been extensively reviewed (Panula et al., 1996; Roumy \& Zajac, 1998; Fukusumi et al., 2006; Yang \& Iadarola, 2006; Yang et al., 2008). NPFF2R mRNA was detected in various brain regions involved in the modulation of nociception such as thalamic nuclei, hypothalamus, and superficial layers of the spinal cord (Bonini et al., 2000; Elshourbagy et al., 2000; Liu et al., 2001; Yang \& Iadarola, 2003). NPFF2R transcripts have also been detected in rat (Bonini et al., 2000; Yang \& Iadarola 2006) and human post-mortem (Yang et al., Neuropeptides 2008) DRGs, as well as in the spinal trigeminal nucleus (STN) of rat (Bonini et al., 2000; Yang \& Iadarola 2003) and primate (Zeng et al., 2003) brain. Human NPFF2R mRNA was also found in the spinal cord, medulla oblongata, hypothalamus, substantia nigra and amygdala (Bonini et al., 2000). Immunohistochemistry and western blotting provided evidence for the presence of NPFF2R in the rat ventral tegmental area (VTA), nucleus accumbens (NAc) and spinal cord (Wu et al., 2010) and for NPFF2R immunoreactivity in the lower brainstem and spinal cord of monkey CNS, with dense labelling in the spinal trigeminal nucleus and superficial layers of the dorsal horn (Zeng et al., 2003). Binding experiments using radiolabelled tracers pointed to the 
presence of NPFF2R binding sites in the superficial layers of the spinal cord, lateral hypothalamus (LHA), parabrachial nucleus (PB), nucleus of solitary tract (NTS) and spinal trigeminal nucleus (STN) in rats and mice (Allard et al., 1989, 1992; Dupouy \& Zajac, 1996; Bonini et al., 2000; Gouarderes et al., 2000, 2002, 2004a, b). Interestingly, pharmacological (neonatal capsaicin treatment) and surgical (dorsal rhizotomy and peripheral axotomy) lesions induced a dramatic decrease in $\left[{ }^{125} \mathrm{I}\right]-(1 \mathrm{DMe}) \mathrm{NPFF}$ binding density in the superficial layers of the dorsal horn of rat spinal cord, suggesting that NPFF2R are partly associated with primary afferents (Gouarderes et al., 1996). Ligation of rat lumbar dorsal spinal roots promoted a net accumulation of receptor sites on the side peripheral to the ligature, as afforded from $\left[{ }^{125} \mathrm{I}\right]-(1 \mathrm{DMe}) \mathrm{NPFF}$ autoradiographic evaluation of NPFF2R sites along ligated root fibers (Gouarderes et al., Synapse 2000). Thus, a population of NPFF2 receptors is synthesized in DRGs and migrates through anterograde axonal transport towards primary afferent endings in the rat spinal cord. Finally, binding experiments conducted on human brain tissues pointed to high densities of $\left[{ }^{125} \mathrm{I}\right]-\mathrm{Y} 8 \mathrm{Fa}$ binding sites in superficial layers of the spinal cord dorsal horn and in the spinal trigeminal nucleus (Allard et al., 1994), though the low NPFF1/2R binding selectivity of Y8Fa (Table 2) precluded a clear definition of the NPFF receptor subtype which was labelled. Noteworthy, within the CNS, highest levels of NPFF1R and NPFF2R mRNA were detected in the human and rat spinal cord, respectively (Bonini et al., 2000; Yang \& Iadarola 2003).

\subsubsection{Modulation of nociception}

Effect of peptide injection: The effects of NPPF and NPAF on nociception have been widely described in several reviews (Panula et al., 1996; Roumy \& Zajac, 1998; Fukusumi et al., 2006; Simonin et al., 2006; Yang \& Iadarola, 2006; Yang et al., 2008; Mouledous et al., 2010b; Ayachi \& Simonin, 2014). NPFF injection was reported to induce two different types of responses depending on the site of injection. In rats or mice, intrathecal NPFF injection evoked analgesia and potentiated opioids action, whereas i.c.v. injection induced pro-nociceptive effects and reversal of morphine analgesia (Roumy \& Zajac, 1998). Anti-morphine analgesic effects of NPFF were blocked by RF9, a selective antagonist of NPFF1/2 receptors, which potentiated opiate-induced analgesia and prevented the development of opiate-induced hyperalgesia and tolerance (Simonin et al., 2006; Elhabazi et al., 2012). Very similarly to NPFF, NPAF administration reversed or potentiated morphine analgesia depending on its route of administration (i.c.v. or i.t., respectively; Bonnard et al., 2001; Jhamandas et al., 2006). Altogether these data indicate that NPFF/NPAF and their NPFF2 receptor contribute to the homeostatic control of opiates action.

Anti opioid effect : To explain tolerance to morphine analgesia and opioid-induced hyperalgesia, the existence of a homeostatic equilibrium between an anti-nociceptive opioid system and a pro- 
nociceptive anti-opioid system has been proposed (Rothman, 1992; Simonnet \& Rivat, 2003). This hypothesis postulates that during opiate treatment, the stimulation of the opioidergic system by exogenous opiates will trigger the release of endogenous pro-nociceptive anti-opioid molecules which will counteract opiates action. Upon chronic opiate administration, anti-opioid molecules levels increase and counteract opiate action, thereby leading to the necessity to increase opiate dosage to reach the same analgesic effect. This may explain the loss of efficacy of opiates, i.e. tolerance to analgesia. Furthermore, when stopping the treatment, the endogenous tone of the antiopioid system remains high, leading to the observation of a decrease in the basal nociceptive threshold and to so-called opioid-induced hyperalgesia. Several lines of evidence support this hypothesis. Particularly, morphine treatments have been shown to induce an increase in NPFF-like immunoreactivity in brain and spinal cord (Malin et al., 1990; Devillers et al., 1995; Stinus et al., 1995) whereas the blockade of NPFF1/2 receptors with an antagonist (RF9) potentiated opiate analgesia and prevented the development of analgesic tolerance and of opioid-induced hyperalgesia (Simonin et al., 2006; Elhabazi et al., 2012). The anti-opioid properties of the NPFF/NPAF system not only concern opiate nociceptive modulating properties but also other opiate effects such as reward, locomotor activity, feeding and drinking behavior (see Section 7.3.2.) or intestinal motility (Mouledous et al., 2010b).

Persistent pain: The implication of NPFF and NPFF2R in persistent pain has been recently reviewed (Ayachi \& Simonin, 2014). In inflammatory pain, several reports indicated that NPFF and NPFF2R were up-regulated at the level of their transcripts (Vilim et al., 1999; Yang \& Iadarola, 2003; Nystedt et al., 2004), binding sites (Lombard et al., 1999) and protein expression, as assessed by NPFF and NPFF2R immunoreactivity (Yang \& Iadarola, 2003; Ayachi \& Simonin, 2014). Conversely, no up-regulation of NPFF and a modest up-regulation of NPFF2R were observed in neuropathic pain conditions (Vilim et al., 1999; Nystedt et al., 2004). It remains that NPFF displayed anti-allodynic and anti-hyperalgesic effects in both models of persistent pain in rodents (Ayachi \& Simonin, 2014). In humans, the concentrations of NPFF in the plasma and in the cerebrospinal fluid of patients with a chronic pain disorder did not differ from those of healthy volunteers, suggesting a lack of up-regulation of this system under persistent pain conditions (Sundblom et al., 1997).

\subsection{The PrRPs/PrRP-R system in nociception and pain}

\subsubsection{Localization in pain pathways}

Figure 9 depicts the distribution of PrRPs and PrRP-R in various nociception and pain modulated areas from rat and mouse central and peripheral nervous system. More details on the distribution of 
PrRPs and PrRP-R in the central nervous system and on their localization in pain pathways are available in (Fukusumi et al., 2006; Lin, 2008; Dodd \& Luckman, 2013; Ayachi \& Simonin, 2014).

In rats, the presence of PrRPs has been demonstrated in several areas implicated in pain control or in processing of nociceptive inputs (Kalliomaki et al., 2004). Indeed, PrRP mRNA was found in the medulla oblongata, nucleus of solitary tract (NTS) and ventrolateral medulla (VLM). PrRP-ir nerve fibers were detected in the VLM, periaqueductal gray (PAG) and basolateral amygdaloid nucleus. No signal was found in the spinal cord, suggesting that pain modulatory effects of PrRPs are probably of supraspinal origin (Kalliomaki et al., 2004). The PrRP receptor was also detected in various regions involved in pain processing including the nucleus tractus solitarius (NTS), lateral and medial parabrachial nuclei (PB), locus coerulus (LC), raphe nucleus (DMR), amygdala (Amd), nucleus accumbens (NAc), and the paraventricular hypothalamic (PVN) nucleus (Fujii et al., 1999; Roland et al., 1999; Fukusumi et al., 2006).

In human brain, PrRP mRNA were found to be enriched in the hypothalamus, medulla oblongata and thalamus (Fujii et al., 1999; Takahashi et al., 2000), in agreement with the involvement of this peptide in the control of pain transmission. In contrast, human PrRP receptor mRNA was detected only in the pituitary gland among tissues examined.

\subsubsection{Modulation of nociception}

PrRPs modulate nociception under basal conditions but in opposite ways, depending on the site of injection. Indeed, when injected directly into the nucleus tractus solitarius (NTS), PrRP-20 induced analgesia (Kalliomaki et al., 2004). When injected in the caudal ventrolateral medulla (VLM) or i.c.v in rats and mice, PrRP promoted hyperalgesia (Kalliomaki et al., 2004; Laurent et al., 2005; Elhabazi et al., 2013). Similarly, under neuropathic pain conditions, PrRPs attenuated tactile allodynia when injected into the PAG or the NTS, but had no effect when injected into the caudal ventrolateral medulla (Kalliomaki et al., 2004).

Likewise the NPFF/NPFF2R system, PrRPs and their receptor have been proposed to belong to an anti-opioid system. Indeed, PrRP KO mice displayed potentiated morphine-induced analgesia, reduced morphine tolerance, enhanced morphine-induced conditioned place preference acquisition and decreased severity of the naloxone-precipitated morphine-withdrawal syndrome, as compared to controls (Laurent et al., 2005). Moreover, the existence of a functional interplay between NPFF1/2 and PrRP receptors has been described. Indeed, the blockade of NPFF1/2 receptors by a selective antagonist (RF9) led to the suppression of the hyperalgesic and anti-morphine effects of PrRPs (Elhabazi et al., 2013), while the anti-opioid effect of NPFF was absent in the PrRP-R KO mice (Laurent et al., 2005). 


\subsection{The kisspeptins/Kiss1R system in nociception and pain}

\subsubsection{Localization in pain pathways}

The distribution of kisspeptins and Kiss1R in several nociception and pain modulating areas of rat and mouse central and peripheral nervous systems is illustrated in Figure 9. Further details on the localization of this peptidergic system in humans and rodents are available in (Kirby et al., 2010).

Kisspeptins are expressed in various pain-related pathways in rodents. Thus, in mouse brain, kisspeptin-ir fibers has been located in the paraventricular hypothalamic nucleus, periaqueductal gray, locus coeruleus and nucleus solitarius (Dun et al., 2003; Clarkson et al., 2009). In the rat, both kisspeptin mRNA and immunoreactivity have been found in the dorsal horn of the spinal cord and in L4/L5 DRG wherein kisspeptin was probably expressed by nociceptors (Dun et al., 2003; Mi et al., 2009). In humans, low levels of KiSS-1 mRNA were found in the brain and more specifically in the hypothalamus and the basal ganglia (Muir et al., 2001), a region known to play a role in pain processing and analgesia.

In rat and mouse brain, Kiss1R was detected in several regions involved in pain control such as the locus coeruleus (LC), parabrachial nuclei (PB), periaqueductal gray (PAG), ventral tegmental area (VTA), amygdala (Amd), lateral hypothalamus area (LHA) and habenula (Hab) (Lee et al., 1999; Herbison et al., 2010). Kiss1R-positive immunostaining has also been found in rat DRG neurons and in lamina I and II of the dorsal horn of the spinal cord (Mi et al., 2009). In humans, Kiss1R mRNA has been detected in many areas implicated in pain and nociception like the amygdala, locus coeruleus, medulla oblongata and spinal cord (Kirby et al., 2010).

\subsubsection{Modulation of nociception}

Kisspeptin displays peripheral pro-nociceptive properties as its intraplantar injection induced a nocifensive response and decreases pain thermal threshold in the hot plate test (Spampinato et al., 2011). At the central level, kisspeptin-10 administration (i.c.v.) induced hyperalgesia as well as anti-morphine activity (Elhabazi et al., 2013). These pro-nociceptive properties also appear to be implicated in inflammatory pain as kisspeptin induced hyperalgesia in formalin test, enhanced TRPV1 phosphorylation at the site of injection and ERK1/2 phosphorylation in the dorsal horn in mice (Spampinato et al., 2011). Kisspeptins and Kiss1R transcripts and proteins were also upregulated in DRG and dorsal horn neurons of rats submitted to the Complete Freund Adjuvant (CFA) model of chronic inflammatory pain (Mi et al., 2009). 


\subsection{The QRFPS/QRFP-R system in nociception and pain}

\subsubsection{Localization in pain pathways}

Figure 9 displays the localization of QRFPs and of QRFP receptors in nociception and pain modulating areas in rats and mice. QRFP mRNA has been mainly detected in the lateral hypothalamic area (Chartrel et al., 2011) but several pieces of evidence also suggest the presence of QRFPs in the rodent spinal cord and DRG (Takayasu et al., 2006; Yamamoto et al., 2008, 2011). QRFP-R1/2 mRNA and binding sites are widely expressed in rodent CNS including in the nucleus solitarius tract (NTS), parabrachial nucleus (PB), locus coerulus (LC), raphe nucleus (DMR), periaqueductal gray (PAG), ventral tegmental area (VTA), amygdala (Amd), habenula (Hab), lateral hypothalamic area (LHA), nucleus accumbens (NAc), paraventricular nucleus of the hypothalamus (PVN) and the spinal cord (Chartrel et al., 2011). In situ hybridization experiments showed that the expression patterns of QRFP-R1 and R2 isoforms in mouse brain did not overlap (Takayasu et al., 2006).

In humans, quantitative PCR analyses pointed to highest expression of QRFP-43 precursor mRNA in the brain, and more precisely in the medulla, retina and vestibular nucleus (Jiang et al., 2003). By using immunohistochemistry and in situ hybridization, Bruzzone and collaborators identified QRFP-26-immunoreactive fibers and QRFP-26-expressing neurons in human paraventricular hypothalamus nucleus and dorsal horn of spinal cord (Bruzzone et al., 2006). In human brain, QRFP receptor mRNA was highly enriched in the trigeminal ganglion, hypothalamus and vestibular nucleus (Jiang et al., 2003).

Overall, QRFP transcript and QRFP-R1/2 binding sites or mRNA were detected in several pain neuronal pathways, raising up their potential involvement in the transmission or integration of nociceptive inputs (Chartrel et al., 2011; Ayachi \& Simonin, 2014).

\subsubsection{Modulation of nociception}

The effects of QRFP peptides on the modulation of nociception have been recently reviewed (Ayachi \& Simonin, 2014). In naive rats or mice, depending on site of application and peptide concentration, QRFP-26 was reported to induce a pro-nociceptive response or no effect on the basal nociceptive threshold (Yamamoto et al., 2008, 2009, 2011; Elhabazi et al., 2013). Conversely to basal nociceptive state, QRFP injection in rats with inflammatory or neuropathic pain produced an anti-allodynic effect (Yamamoto et al., 2008, 2009, 2011).

To conclude this section, the distribution pattern and/or in vivo activity of all RF-amide peptides and their receptors attest of their involvement in the modulation of nociception, both under basal or 
chronic pain conditions. Moreover, several RF-amide peptides and their receptors belong to an antiopioid pro-nociceptive system that is prone to modulate opiates action. All these data emphasize the importance of these RF-amide systems in the modulation of nociception and opiate analgesia and the need to develop alternative therapeutic approaches for pain treatment.

\section{Conclusion and therapeutic perspectives}

Since the discovery of the first mammalian RF-amide peptide NPFF (Yang et al., 1985), thirty years of research efforts have led to the identification of a rather large family of mammalian neuropeptides and their receptors. These neuropeptide signaling systems seem to be conserved during the course of evolution, not only at a structural level, but also functionally. Their wellestablished occurrence prior to the divergence of protostomes and deuterostomes emphasizes the importance of these systems in both Vertebrates and Invertebrates. In Mammals, functional studies support the evolutionary continuity of RF-amides as key regulators of energy balance, feeding behavior, reproduction, and sensory modulation. Moreover, their distribution both at the spinal and supraspinal levels as well as at the periphery clearly reflects their involvement in these functions. From a philogenetic point of view, kisspeptin and its receptor separate from the other RF-amide systems. However, it is interesting to note that galanin receptors and their ligands that cluster with kisspeptin ones have also been clearly involved in energy homeostasis, pain and reproduction (Lang et al., 2014; Fang et al., 2015), suggesting that the galanin system should also be considered when studying the function of RF-amide peptides and their receptors.

From a therapeuthic perspective, although these different mammalian RF-amide systems have been involved in the regulation of the same functions, some of them are emerging as interesting targets for specific applications. NPFF/NPFF2R seems to be the most promising pair to target for pain treatment. Indeed, NPFF2R antagonists may be regarded as innovative tools to improve the efficacy and limit the undesirable side effects of opiates during chronic pain therapy (Elhabazi et al., 2012). Moreover, looking at single-nucleotide polymorphisms in the NPFF2R gene, some haplotypes have been associated with an increased risk of obesity whereas others were strongly protective against obesity, in association with higher lipolytic activity (Dahlman et al., 2007; Hunt et al., 2011), suggesting that NPFF2R may play an important role in obesity predisposition. Kisspeptin/Kiss1R and to a lower extent RFRP/NPFF1R represent the most promising members of the RF-amide family to control reproduction and fertility both in animals and humans. Kisspeptin receptor agonists could be helpful in improving the reproductive activity of domestic animals, including stimulation of quiescent gonadotropin axis and optimization of artificial insemination (Caraty et al., 2012b), as well as in the treatment of fertility disorders in 
women (Clarke et al., 2015). Furthermore, the recent discovery of a hormonal kisspeptin circuit between the liver and the endocrine pancreas highlighted a link between hyperglucagonemia, increased hepatic kisspeptin production and impaired glucose-stimulated insulin secretion from $\beta$ pancreatic cells in type 2 diabetes mellitus (Song et al., 2014). These observations suggest that peripherally-acting kisspeptin receptor antagonists could represent interesting therapeutic tools for the treatment of type 2 diabetes (Song et al., 2014). Targeting QRFP/QRFPR and PrRP/PrRPR systems appears more promising in the control of feeding behaviour. Indeed, QRFPs have been shown to be regulated by adiposity signals and nutrients status and to be orexigenic in several mammalian species. Therefore, elucidating the mechanisms by which QRFP receptor agonists increase high-fat diet intake could markedly influence the development of therapies for the treatment of obesity (Primeaux et al., 2013). The PrRP system has been shown to be an important point of integration in the control of energy metabolism and stress response, suggesting that this anorexigenic system might also represent an interesting target for the treatment of obesity (Onaka et al., 2010).

In conclusion, the mammalian RF-amide system shares many features for a family that includes not only peptides with a conserved Arg-Phe- $\mathrm{NH}_{2}$ sequence at their carboxyl-terminal end but also receptors that display significant sequence homologies and regulate the same set of physiological functions. Strikingly, endogenous opioid peptides and their receptors play prominent roles in the same functions, suggesting a close link between opioid and RF-amide systems. Such a relationship has been pointed very early for NPFF, with the proposal of an anti-opioid function that has been largely characterized in subsequent studies. Futher progress in the development of selective pharmacological tools for the different RF-amide receptors should greatly facilitate the study of their function in various mammalian species and confirm their potential as therapeutic targets.

\section{Conflict of interest}

The authors declare no conflicts of interest.

\section{Acknowledgments}

This work was supported by the CNRS, INSERM, Université de Strasbourg and by grants from Conectus and the Agence Nationale de la Recherche (ANR-13-BSV1-0001). This work has been published within the LABEX ANR-10-LABX-0034-Medalis and received financial support from the French government managed by "Agence Nationale de la Recherche" under "Programme d'investissement d'avenir. 


\section{References}

Abbara A., Jayasena C.N., Christopoulos G., Narayanaswamy S., Izzi-Engbeaya C., Nijher G.M.K., et al. (2015). Efficacy of Kisspeptin-54 to Trigger Oocyte Maturation in Women at High Risk of Ovarian Hyperstimulation Syndrome (OHSS) During In Vitro Fertilization (IVF) Therapy. J Clin Endocrinol Metab 100, 3322-3331.

Acosta-Galvan G., Yi C.X., van der Vliet J., Jhamandas J.H., Panula P., Angeles-Castellanos M., et al. (2011). Interaction between hypothalamic dorsomedial nucleus and the suprachiasmatic nucleus determines intensity of food anticipatory behavior. Proc Natl Acad Sci U S A 108, 5813-5818.

Adachi S., Yamada S., Takatsu Y., Matsui H., Kinoshita M., Takase K., et al. (2007). Involvement of anteroventral periventricular metastin/kisspeptin neurons in estrogen positive feedback action on luteinizing hormone release in female rats. J Reprod Dev 53, 367-378.

Ahmed M.L., Ong K.K. \& Dunger D.B. (2009). Childhood obesity and the timing of puberty. Trends Endocrinol Metab 20, 237-242.

Ahow M., Min L., Pampillo M., Nash C., Wen J., Soltis K., et al. (2014). KISS1R signals independently of Galphaq/11 and triggers LH secretion via the beta-arrestin pathway in the male mouse. Endocrinology 155, 4433-4446.

Aiglstorfer I., Hendrich I., Moser C., Bernhardt G., Dove S. \& Buschauer A. (2000). Structure-Activity Relationships of Neuropeptide Y Y1 Receptor Antagonists Related to BIBP 3226. Bioorganic \& Medicinal Chemistry Letters 10, 1597-1600.

Allard M., Geoffre S., Legendre P., Vincent J.D. \& Simonnet G. (1989). Characterization of rat spinal cord receptors to FLFQPQRF-amide, a mammalian morphine modulating peptide: a binding study. Brain Res 500, $169-176$.

Allard M., Zajac J.M. \& Simonnet G. (1992). Autoradiographic distribution of receptors to FLFQPQRF-amide, a morphine-modulating peptide, in rat central nervous system. Neuroscience 49, 101-116.

Allard M., Jordan D., Zajac J.M., Ries C., Martin D., Monkouanga D., et al. (1994). Autoradiographic localization of receptors for neuropeptide FF, FLFQPQRF-amide, in human spinal sensory system. Brain Res 633, 127-132.

Ancel C., Bentsen A.H., Sebert M.E., Tena-Sempere M., Mikkelsen J.D. \& Simonneaux V. (2012). Stimulatory effect of RFRP-3 on the gonadotrophic axis in the male Syrian hamster: the exception proves the rule. Endocrinology 153, 1352-1363.

Anderson E.K., Gutierrez D.A. \& Hasty A.H. (2010). Adipose tissue recruitment of leukocytes. Curr Opin Lipidol 21, 172-177.

Anderson G.M., Relf H.L., Rizwan M.Z. \& Evans J.J. (2009). Central and peripheral effects of RF-amide-related peptide-3 on luteinizing hormone and prolactin secretion in rats. Endocrinology 150, 1834-1840.

Andreu C., Parborell F., Vanzulli S., Chemes H. \& Tesone M. (1998). Regulation of follicular luteinization by a gonadotropin-releasing hormone agonist: relationship between steroidogenesis and apoptosis. Mol Reprod Dev 51, 287-294.

Anjum S., Krishna A., Sridaran R. \& Tsutsui K. (2012). Localization of gonadotropin-releasing hormone $(\mathrm{GnRH})$, gonadotropin-inhibitory hormone $(\mathrm{GnIH})$, kisspeptin and $\mathrm{GnRH}$ receptor and their possible roles in testicular activities from birth to senescence in mice. J Exp Zool A Ecol Genet Physiol 317, 630-644.

Anjum S., Krishna A. \& Tsutsui K. (2014). Inhibitory roles of the mammalian GnIH ortholog RFRP3 in testicular activities in adult mice. $J$ Endocrinol 223, 79-91.

Anko M.L., Ostergard M., Lintunen M. \& Panula P. (2006). Alternative splicing of human and mouse NPFF2 receptor genes: Implications to receptor expression. FEBS Lett 580, 6955-6960.

Anko M.L. \& Panula P. (2006). Regulation of endogenous human NPFF2 receptor by neuropeptide FF in SK-NMC neuroblastoma cell line. J Neurochem 96, 573-584.

Ansel L., Bolborea M., Bentsen A.H., Klosen P., Mikkelsen J.D. \& Simonneaux V. (2010). Differential regulation of kiss1 expression by melatonin and gonadal hormones in male and female Syrian hamsters. $J$ Biol Rhythms 25, 81-91.

Ansel L., Bentsen A.H., Ancel C., Bolborea M., Klosen P., Mikkelsen J.D., et al. (2011). Peripheral kisspeptin reverses short photoperiod-induced gonadal regression in Syrian hamsters by promoting GNRH release. Reproduction 142, 417-425.

Arison R.N., Ciaccio E.I., Glitzer M.S., Cassaro J.A. \& Pruss M.P. (1967). Light and electron microscopy of lesions in rats rendered diabetic with streptozotocin. Diabetes 16, 51-56.

Asami T., Nishizawa N., Ishibashi Y., Nishibori K., Horikoshi Y., Matsumoto H., et al. (2012a). Trypsin resistance of a decapeptide KISS1R agonist containing an N-omega-methylarginine substitution. Bioorganic \& Medicinal Chemistry Letters 22, 6328-6332. 
Asami T., Nishizawa N., Ishibashi Y., Nishibori K., Nakayama M., Horikoshi Y., et al. (2012b). Serum stability of selected decapeptide agonists of KISS1R using pseudopeptides. Bioorganic \& Medicinal Chemistry Letters 22, 6391-6396.

Asami T., Nishizawa N., Matsui H., Nishibori K., Ishibashi Y., Horikoshi Y., et al. (2013). Design, Synthesis, and Biological Evaluation of Novel Investigational Nonapeptide KISS1R Agonists with TestosteroneSuppressive Activity. J Med Chem 56, 8298-8307.

Asami T., Nishizawa N., Matsui H., Takatsu Y., Suzuki A., Kiba A., et al. (2014). Physicochemically and Pharmacokinetically Stable Nonapeptide KISS1 Receptor Agonists with Highly Potent TestosteroneSuppressive Activity. Journal of Medicinal Chemistry 57, 6105-6115.

Avena N.M. \& Bocarsly M.E. (2012). Dysregulation of brain reward systems in eating disorders: neurochemical information from animal models of binge eating, bulimia nervosa, and anorexia nervosa. Neuropharmacology 63, 87-96.

Ayachi S. \& Simonin F. (2014). Involvement of Mammalian RF-Amide Peptides and Their Receptors in the Modulation of Nociception in Rodents. Front Endocrinol (Lausanne) 5, 158.

Babwah A.V., Navarro V.M., Ahow M., Pampillo M., Nash C., Fayazi M., et al. (2015). GnRH Neuron-Specific Ablation of Gaq/11 Results in Only Partial Inactivation of the Neuroendocrine-Reproductive Axis in Both Male and Female Mice: In Vivo Evidence for Kiss1r-Coupled Gaq/11-Independent GnRH Secretion. J Neurosci 35, 12903-12916.

Backholer K., Smith J.T., Rao A., Pereira A., Iqbal J., Ogawa S., et al. (2010). Kisspeptin cells in the ewe brain respond to leptin and communicate with neuropeptide $\mathrm{Y}$ and proopiomelanocortin cells. Endocrinology 151, 2233-2243.

Banks W.J. (1993). Female reproductive system. (Eds.),Applied veterinary histology(pp. 452-457). St Louis: Mosby-Year book Inc.

Baribault H., Danao J., Gupte J., Yang L., Sun B., Richards W., et al. (2006). The G-protein-coupled receptor GPR103 regulates bone formation. Mol Cell Biol 26, 709-717.

Barsh G.S. \& Schwartz M.W. (2002). Genetic approaches to studying energy balance: perception and integration. Nat Rev Genet 3, 589-600.

Bartzen-Sprauer J., Klosen P., Ciofi P., Mikkelsen J.D. \& Simonneaux V. (2014). Photoperiodic co-regulation of kisseptin, neurokinin B and dynorphin in the hypothalamus of a seasonal rodent. J Neuroendocrinol 26, 510520.

Bechtold D.A. \& Luckman S.M. (2006). Prolactin-releasing Peptide mediates cholecystokinin-induced satiety in mice. Endocrinology 147, 4723-4729.

Bechtold D.A. \& Luckman S.M. (2007). The role of RF-amide peptides in feeding. J Endocrinol 192, 3-15.

Beck B. \& Richy S. (2009). Suppression of QRFP 43 in the hypothalamic ventromedial nucleus of Long-Evans rats fed a high-fat diet. Biochem Biophys Res Commun 383, 78-82.

Becker J.A., Mirjolet J.F., Bernard J., Burgeon E., Simons M.J., Vassart G., et al. (2005). Activation of GPR54 promotes cell cycle arrest and apoptosis of human tumor cells through a specific transcriptional program not shared by other Gq-coupled receptors. Biochem Biophys Res Commun 326, 677-686.

Beltramo M., Robert V., Galibert M., Madinier J-B., Marceau P., Dardente H., et al. (2015). Rational Design of Triazololipopeptides Analogs of Kisspeptin Inducing a Long-Lasting Increase of Gonadotropins. J Med Chem 58, 3459-3470.

Bihel F., Humbert J.P., Schneider S., Bertin I., Wagner P., Schmitt M., et al. (2015). Development of a peptidomimetic antagonist of neuropeptide FF receptors for the prevention of opioid-induced hyperalgesia. ACS Chem Neurosci.

Bilban M., Ghaffari-Tabrizi N., Hintermann E., Bauer S., Molzer S., Zoratti C., et al. (2004). Kisspeptin-10, a KiSS-1/metastin-derived decapeptide, is a physiological invasion inhibitor of primary human trophoblasts. $J$ Cell Sci 117, 1319-1328.

Biro F.M. \& Wien M. (2010). Childhood obesity and adult morbidities. Am J Clin Nutr 91, 1499S-1505S.

Bjursell M., Lenneras M., Goransson M., Elmgren A. \& Bohlooly Y.M. (2007). GPR10 deficiency in mice results in altered energy expenditure and obesity. Biochem Biophys Res Commun 363, 633-638.

Boer H.H., Schot L.P., Veenstra J.A. \& Reichelt D. (1980). Immunocytochemical identification of neural elements in the central nervous systems of a snail, some insects, a fish, and a mammal with an antiserum to the molluscan cardio-excitatory tetrapeptide FMRF-amide. Cell Tissue Res 213, 21-27.

Boersma C.J., Sonnemans M.A. \& Van Leeuwen F.W. (1993). Immunocytochemical localization of neuropeptide FF (FMRF amide-like peptide) in the hypothalamo-neurohypophyseal system of Wistar and Brattleboro rats by light and electron microscopy. J Comp Neurol 336, 555-570.

Bonini J.A., Jones K.A., Adham N., Forray C., Artymyshyn R., Durkin M.M., et al. (2000). Identification and characterization of two G protein-coupled receptors for neuropeptide FF. J Biol Chem 275, 39324-39331.

Bonnard E., Burlet-Schiltz O., Frances B., Mazarguil H., Monsarrat B., Zajac J.M., et al. (2001). Identification of neuropeptide FF-related peptides in rodent spinal cord. Peptides 22, 1085-1092. 
Bonnard E., Burlet-Schiltz O., Monsarrat B., Girard J.P. \& Zajac J.M. (2003). Identification of proNeuropeptide FFA peptides processed in neuronal and non-neuronal cells and in nervous tissue. Eur J Biochem 270, 41874199.

Bosch M.A., Xue C. \& Ronnekleiv O.K. (2012). Kisspeptin expression in guinea pig hypothalamus: effects of 17beta-estradiol. J Comp Neurol 520, 2143-2162.

Boyle R.G., Downham R., Ganguly T., Humphries J., Smith J. \& Travers S. (2005). Structure-activity studies on prolactin-releasing peptide (PrRP). Analogues of PrRP-(19-31)-peptide. Journal of Peptide Science 11, 161165.

Brailoiu G.C., Dun S.L., Ohsawa M., Yin D., Yang J., Chang J.K., et al. (2005). KiSS-1 expression and metastin-like immunoreactivity in the rat brain. J Comp Neurol 481, 314-329.

Brown R.E., Imran S.A., Ur E. \& Wilkinson M. (2008). KiSS-1 mRNA in adipose tissue is regulated by sex hormones and food intake. Mol Cell Endocrinol 281, 64-72.

Brussaard A.B., Kits K.S., Termaat A., Mulder A.H. \& Schoffelmeer A.N.M. (1989). Peripheral Injection of Dns-Rfa, a Fmrfa Agonist, Suppresses Morphine-Induced Analgesia in Rats. Peptides 10, 735-739.

Bruzzone F., Lectez B., Tollemer H., Leprince J., Dujardin C., Rachidi W., et al. (2006). Anatomical distribution and biochemical characterization of the novel RF-amide peptide $26 \mathrm{RFa}$ in the human hypothalamus and spinal cord. J Neurochem 99, 616-627.

Bruzzone F., Lectez B., Alexandre D., Jegou S., Mounien L., Tollemer H., et al. (2007). Distribution of 26RFa binding sites and GPR103 mRNA in the central nervous system of the rat. J Comp Neurol 503, 573-591.

Burgus R., Butcher M., Amoss M., Ling N., Monahan M., Rivier J., et al. (1972). Primary structure of the ovine hypothalamic luteinizing hormone-releasing factor (LRF) (LH-hypothalamus-LRF-gas chromatography-mass spectrometry-decapeptide-Edman degradation). Proc Natl Acad Sci U S A 69, 278-282.

Burlet-Schiltz O., Mazarguil H., Sol J.C., Chaynes P., Monsarrat B., Zajac J.M., et al. (2002). Identification of neuropeptide FF-related peptides in human cerebrospinal fluid by mass spectrometry. FEBS Lett 532, 313318.

Caba M., Pabello M., Moreno M.L. \& Meza E. (2014). Main and accessory olfactory bulbs and their projections in the brain anticipate feeding in food-entrained rats. Chronobiol Int 31, 869-877.

Cani P.D., Bibiloni R., Knauf C., Waget A., Neyrinck A.M., Delzenne N.M., et al. (2008). Changes in gut microbiota control metabolic endotoxemia-induced inflammation in high-fat diet-induced obesity and diabetes in mice. Diabetes 57, 1470-1481.

Caraty A., Smith J.T., Lomet D., Ben Said S., Morrissey A., Cognie J., et al. (2007). Kisspeptin synchronizes preovulatory surges in cyclical ewes and causes ovulation in seasonally acyclic ewes. Endocrinology 148, 5258-5267.

Caraty A., Blomenröhrt M., Vogel G.M.T., Lomet D., Briant C. \& Beltramo M. (2012a). RF9 Powerfully Stimulates Gonadotrophin Secretion in the Ewe: Evidence for a Seasonal Threshold of Sensitivity. $J$ Neuroendocrinol 24, 725-736.

Caraty A., Decourt C., Briant C. \& Beltramo M. (2012b). Kisspeptins and the reproductive axis: potential applications to manage reproduction in farm animals. Domest Anim Endocrinol 43, 95-102.

Caroff E., Steger M., Valdenaire O., Fecher A., Breu V., Hilpert K., et al. (2004). Preparation of tetrahydrobenzothiazolylguanidines and related compounds as neuropeptide FF receptor antagonists, (Ltd, A. P. ed).

Castano J.P., Martinez-Fuentes A.J., Gutierrez-Pascual E., Vaudry H., Tena-Sempere M. \& Malagon M.M. (2009). Intracellular signaling pathways activated by kisspeptins through GPR54: do multiple signals underlie function diversity? Peptides 30, 10-15.

Castellano J.M., Navarro V.M., Fernandez-Fernandez R., Nogueiras R., Tovar S., Roa J., et al. (2005). Changes in hypothalamic KiSS-1 system and restoration of pubertal activation of the reproductive axis by kisspeptin in undernutrition. Endocrinology 146, 3917-3925.

Castellano J.M., Navarro V.M., Fernandez-Fernandez R., Castano J.P., Malagon M.M., Aguilar E., et al. (2006a). Ontogeny and mechanisms of action for the stimulatory effect of kisspeptin on gonadotropinreleasing hormone system of the rat. Mol Cell Endocrinol 257i 258, 75 ï 83.

Castellano J.M., Navarro V.M., Fernandez-Fernandez R., Roa J., Vigo E., Pineda R., et al. (2006b). Expression of hypothalamic KiSS-1 system and rescue of defective gonadotropic responses by kisspeptin in streptozotocin-induced diabetic male rats. Diabetes 55, 2602-2610.

Castellano J.M., Navarro V.M., Roa J., Pineda R., Sanchez-Garrido M.A., Garcia-Galiano D., et al. (2009a). Alterations in hypothalamic KiSS-1 system in experimental diabetes: early changes and functional consequences. Endocrinology 150, 784-794.

Castellano J.M., Roa J., Luque R.M., Dieguez C., Aguilar E., Pinilla L., et al. (2009b). KiSS-1/kisspeptins and the metabolic control of reproduction: physiologic roles and putative physiopathological implications. Peptides 30, 139-145. 
Castellano J.M., Bentsen A.H., Mikkelsen J.D. \& Tena-Sempere M. (2010a). Kisspeptins: bridging energy homeostasis and reproduction. Brain Res 1364, 129-138.

Castellano J.M., Bentsen A.H., Romero M., Pineda R., Ruiz-Pino F., Garcia-Galiano D., et al. (2010b). Acute inflammation reduces kisspeptin immunoreactivity at the arcuate nucleus and decreases responsiveness to kisspeptin independently of its anorectic effects. Am J Physiol Endocrinol Metab 299, E54-61.

Castellano J.M., Bentsen A.H., Sanchez-Garrido M.A., Ruiz-Pino F., Romero M., Garcia-Galiano D., et al. (2011). Early metabolic programming of puberty onset: impact of changes in postnatal feeding and rearing conditions on the timing of puberty and development of the hypothalamic kisspeptin system. Endocrinology 152, 3396-3408.

Chamberlin N.L., Mansour A., Watson S.J. \& Saper C.B. (1999). Localization of mu-opioid receptors on amygdaloid projection neurons in the parabrachial nucleus of the rat. Brain Res 827, 198-204.

Chan Y-M., Butler J.P., Pinnell N.E., Pralong F.P., Crowley W.F. Jr., Ren C., et al. (2011). Kisspeptin Resets the Hypothalamic GnRH Clock in Men. J Clin Endocrinol Metab 96, E908-E915.

Chartrel N., Dujardin C., Anouar Y., Leprince J., Decker A., Clerens S., et al. (2003). Identification of 26RFa, a hypothalamic neuropeptide of the RF-amide peptide family with orexigenic activity. Proc Natl Acad Sci U S A $100,15247-15252$.

Chartrel N., Bruzzone F., Leprince J., Tollemer H., Anouar Y., Do-Rego J.C., et al. (2006). Structure and functions of the novel hypothalamic RF-amide neuropeptides R-RFa and 26RFa in vertebrates. Peptides 27, 1110-1120.

Chartrel N., Alonzeau J., Alexandre D., Jeandel L., Alvear-Perez R., Leprince J., et al. (2011). The RF-amide neuropeptide $26 \mathrm{RFa}$ and its role in the control of neuroendocrine functions. Front Neuroendocrinol 32, 387397.

Chen C., Dun S.L., Dun N.J. \& Chang J.K. (1999). Prolactin-releasing peptide-immunoreactivity in A1 and A2 noradrenergic neurons of the rat medulla. Brain Res 822, 276-279.

Chen J., Fu, R., Cui Y., Pan J., Li Y., Zhang X., et al. (2014). LIM-Homeodomain Transcription Factor Isl-1 Mediates Kisspeptinôs Effect on Insulin Secretion in Mice. Mol Endocrinol 28, 1276-1290.

Cheng G., Coolen L.M., Padmanabhan V., Goodman R.L. \& Lehman M.N. (2010). The kisspeptin/neurokinin B/dynorphin (KNDy) cell population of the arcuate nucleus: sex differences and effects of prenatal testosterone in sheep. Endocrinology 151, 301-311.

Cikos S., Gregor P. \& Koppel J. (1999). Sequence and tissue distribution of a novel G-protein-coupled receptor expressed prominently in human placenta. Biochem Biophys Res Commun 256, 352-356.

Clapham D.E. \& Neer E.J. (1997). G Protein $\beta \gamma$ Subunits. Annu Rev Pharmacol Toxicol 37, 167-203.

Clarke H., Dhillo W.S. \& Jayasena C.N. (2015). Comprehensive Review on Kisspeptin and Its Role in Reproductive Disorders. Endocrinol Metab 30, 124-141.

Clarke I.J., Sari I.P., Qi Y., Smith J.T., Parkington H.C., Ubuka T., et al. (2008). Potent action of RF-amiderelated peptide-3 on pituitary gonadotropes indicative of a hypophysiotropic role in the negative regulation of gonadotropin secretion. Endocrinology 149, 5811-5821.

Clarke I.J., Smith J.T., Henry B.A., Stefanidis A., Millar R.P., Sari I.P., et al. (2012). Gonadotropin-inhibitory hormone is a hypothalamic peptide that provides a molecular switch between reproduction and feeding. Neuroendocrinology 95, 305-316.

Clarkson J. \& Herbison A.E. (2006). Postnatal development of kisspeptin neurons in mouse hypothalamus; sexual dimorphism and projections to gonadotropin-releasing hormone neurons. Endocrinology 147, 58175825 .

Clarkson J., d'Anglemont de Tassigny X., Colledge W.H., Caraty A. \& Herbison A.E. (2009). Distribution of kisspeptin neurones in the adult female mouse brain. J Neuroendocrinol 21, 673-682.

Cleary M.P., Bergstrom H.M., Dodge T.L., Getzin S.C., Jacobson M.K. \& Phillips F.C. (2001). Restoration of fertility in young obese (Lep(ob) Lep(ob)) male mice with low dose recombinant mouse leptin treatment. Int J Obes Relat Metab Disord 25, 95-97.

Colledge W.H. (2009). Kisspeptins and GnRH neuronal signalling. Trends Endocrinol Metab 20, 115-121.

Connolly P.B. \& Resko J.A. (1994). Prenatal testosterone differentiates brain regions controlling gonadotropin release in guinea pigs. Biol Reprod 51, 125-130.

Corbier P. (1985). Sexual differentiation of positive feedback: effect of hour of castration at birth on estradiolinduced luteinizing hormone secretion in immature male rats. Endocrinology 116, 142-147.

Cravo R.M., Margatho L.O., Osborne-Lawrence S., Donato J., Jr., Atkin S., Bookout A.L., et al. (2011). Characterization of Kiss 1 neurons using transgenic mouse models. Neuroscience 173, 37-56.

Crawley J.N. \& Beinfeld M.C. (1983). Rapid development of tolerance to the behavioural actions of cholecystokinin. Nature 302, 703-706.

Curtis A.E., Cooke J.H., Baxter J.E., Parkinson J.R.C., Bataveljic A., Ghatei M.A., et al. (2010). A kisspeptin-10 analog with greater in vivo bioactivity than kisspeptin-10. Am J Physiol Endocrinol Metab 298, E296-E303. 
Cvetkovic D., Babwah A.V. \& Bhattacharya M. (2013). Kisspeptin/KISS1R System in Breast Cancer. J Cancer 4, 653-661.

d'Anglemont de Tassigny X., Fagg L.A., Dixon J.P., Day K., Leitch H.G., Hendrick A.G., et al. (2007). Hypogonadotropic hypogonadism in mice lacking a functional Kiss1 gene. Proc Natl Acad Sci U S A 104, 10714-10719.

d'Anglemont de Tassigny X., Fagg L.A., Carlton M.B. \& Colledge W.H. (2008). Kisspeptin can stimulate gonadotropin-releasing hormone $(\mathrm{GnRH})$ release by a direct action at $\mathrm{GnRH}$ nerve terminals. Endocrinology 149, 3926-3932.

d'Anglemont de Tassigny X. \& Colledge W.H. (2010). The role of kisspeptin signaling in reproduction. Physiology (Bethesda) 25, 207-217.

D'Ursi A.M., Albrizio S., Di Fenza A., Crescenzi O., Carotenuto A., Picone D., et al. (2002). Structural studies on Hgr3 orphan receptor ligand prolactin-releasing peptide. Journal of Medicinal Chemistry 45, 5483-5491.

Dahlman I., Dicker A., Jiao H., Kere J., Blomqvist L., van Harmelen V., et al. (2007). A common haplotype in the G-protein-coupled receptor gene GPR74 is associated with leanness and increased lipolysis. Am J Hum Genet 80, 1115-1124.

Dardeno T.A., Chou S.H., Moon H.S., Chamberland J.P., Fiorenza C.G. \& Mantzoros C.S. (2010). Leptin in human physiology and therapeutics. Front Neuroendocrinol 31, 377-393.

Dardente H., Klosen P., Pevet P. \& Masson-Pevet M. (2003). MT1 melatonin receptor mRNA expressing cells in the pars tuberalis of the European hamster: effect of photoperiod. J Neuroendocrinol 15, 778-786.

Dardente H., Birnie M., Lincoln G.A. \& Hazlerigg D.G. (2008). RF-amide-related peptide and its cognate receptor in the sheep: cDNA cloning, mRNA distribution in the hypothalamus and the effect of photoperiod. J Neuroendocrinol 20, 1252-1259.

De Bond J.A. \& Smith J.T. (2014). Kisspeptin and energy balance in reproduction. Reproduction 147, R53-63.

de Roux N., Genin E., Carel J.C., Matsuda F., Chaussain J.L. \& Milgrom E. (2003). Hypogonadotropic hypogonadism due to loss of function of the KiSS1-derived peptide receptor GPR54. Proc Natl Acad Sci U S A 100, 10972-10976.

Decker B., Vadokas B., Kutschenreuter U., Golenhofen K., Voigt K., McGregor G.P., et al. (1997). Action of FMRF-amide-like peptides on porcine gastrointestinal motility in vitro. Peptides 18, 1531-1537.

Decourt C., Tillet Y., Caraty A., Franceschini I. \& Briant C. (2008). Kisspeptin immunoreactive neurons in the equine hypothalamus Interactions with GnRH neuronal system. J Chem Neuroanat 36, 131-137.

Defea K. (2008). Beta-arrestins and heterotrimeric G-proteins: collaborators and competitors in signal transduction. Br J Pharmacol 153 Suppl 1, S298-309.

DeLuca S.H., Rathmann D., Beck-Sickinger A.G. \& Meiler J. (2013). The activity of prolactin releasing peptide correlates with its helicity. Biopolymers 99, 314-325.

Desprat C. \& Zajac J.M. (1997). Hypothermic effects of neuropeptide FF analogues in mice. Pharmacol Biochem Behav 58, 559-563.

Desroziers E., Mikkelsen J., Simonneaux V., Keller M., Tillet Y., Caraty A., et al. (2010). Mapping of kisspeptin fibres in the brain of the pro-oestrous rat. J Neuroendocrinol 22, 1101-1112.

Devillers J.P., Mazarguil H., Allard M., Dickenson A.H., Zajac J.M. \& Simonnet G. (1994). Characterization of a potent agonist for NPFF receptors: binding study on rat spinal cord membranes. Neuropharmacology 33, 661-669.

Devillers J.P., Boisserie F., Laulin J.P., Larcher A. \& Simonnet G. (1995). Simultaneous activation of spinal antiopioid system (neuropeptide FF) and pain facilitatory circuitry by stimulation of opioid receptors in rats. Brain Res 700, 173-181.

Dhillo W.S., Chaudhri O.B., Patterson M., Thompson E.L., Murphy K.G., Badman M.K., et al. (2005). Kisspeptin-54 stimulates the hypothalamic-pituitary gonadal axis in human males. J Clin Endocrinol Metab 90, 6609-6615.

Dhillo W.S., Chaudhri O.B., Thompson E.L., Murphy K.G., Patterson M., Ramachandran R., et al. (2007). Kisspeptin-54 stimulates gonadotropin release most potently during the preovulatory phase of the menstrual cycle in women. J Clin Endocrinol Metab 92, 3958-3966.

Dina C., Meyre D., Gallina S., Durand E., Korner A., Jacobson P., et al. (2007). Variation in FTO contributes to childhood obesity and severe adult obesity. Nat Genet 39, 724-726.

Do E.U., Choi G., Shin J., Jung W.S. \& Kim S.I. (2004). Fluorescence polarization assays for high-throughput screening of neuropeptide FF receptors. Anal Biochem 330, 156-163.

Do E.U., Piao L.Z., Choi G., Choi Y.B., Kang T.M., Shin J., et al. (2006). The high throughput screening of neuropeptide FF2 receptor ligands from Korean herbal plant extracts. Peptides 27, 997-1004.

do Rego J.C., Leprince J., Chartrel N., Vaudry H. \& Costentin J. (2006). Behavioral effects of 26RF-amide and related peptides. Peptides 27, 2715-2721.

Dockray G.J., Vaillant C. \& Williams R.G. (1981). New vertebrate brain--gut peptide related to a molluscan neuropeptide and an opioid peptide. Nature 293, 656-657. 
Dockray G.J., Reeve J.R., Jr., Shively J., Gayton R.J. \& Barnard C.S. (1983). A novel active pentapeptide from chicken brain identified by antibodies to FMRF-amide. Nature 305, 328-330.

Dockray G.J. (2004). The expanding family of -RF-amide peptides and their effects on feeding behaviour. Exp Physiol 89, 229-235.

Dodd G.T. \& Luckman S.M. (2013). Physiological Roles of GPR10 and PrRP Signaling. Front Endocrinol (Lausanne) 4, 20.

Doebelin C., Wagner P., Bertin I., Simonin F., Schmitt M., Bihel F., et al. (2013). Trisubstitution of pyridine through sequential and regioselective palladium cross-coupling reactions affording analogs of known GPR54 antagonists. Rsc Advances 3, 10296-10300.

Ducret E., Anderson G.M. \& Herbison A.E. (2009). RF-amide-related peptide-3, a mammalian gonadotropininhibitory hormone ortholog, regulates gonadotropin-releasing hormone neuron firing in the mouse. Endocrinology 150, 2799-2804.

Dumalska I., Wu M., Morozova E., Liu R., van den Pol A. \& Alreja M. (2008). Excitatory Effects of the Puberty-Initiating Peptide Kisspeptin and Group I Metabotropic Glutamate Receptor Agonists Differentiate Two Distinct Subpopulations of Gonadotropin-Releasing Hormone Neurons. J Neurosci 28, 8003-8013.

Dun S.L., Brailoiu G.C., Parsons A., Yang J., Zeng Q., Chen X., et al. (2003). Metastin-like immunoreactivity in the rat medulla oblongata and spinal cord. Neurosci Lett 335, 197-201.

Dungan H.M., Gottsch M.L., Zeng H., Gragerov A., Bergmann J.E., Vassilatis D.K., et al. (2007). The role of kisspeptin-GPR54 signaling in the tonic regulation and surge release of gonadotropin-releasing hormone/luteinizing hormone. J Neurosci 27, 12088-12095.

Dupouy V., Puget A., Eschalier A. \& Zajac J.M. (1996). Species differences in the localization of neuropeptide FF receptors in rodent and lagomorph brain and spinal cord. Peptides 17, 399-405.

Dupouy V. \& Zajac J.M. (1996). Neuropeptide FF receptors in rat brain: a quantitative light-microscopic autoradiographic study using [125I][D.Tyr1, (NMe)Phe3]NPFF. Synapse 24, 282-296.

Egido E.M., Hernandez R., Leprince J., Chartrel N., Vaudry H., Marco J., et al. (2007). 26RFa, a novel orexigenic neuropeptide, inhibits insulin secretion in the rat pancreas. Peptides 28, 725-730.

Eipper B.A., Stoffers D.A. \& Mains R.E. (1992). The biosynthesis of neuropeptides: peptide alpha-amidation. Annu Rev Neurosci 15, 57-85.

Elhabazi K., Trigo J.M., Mollereau C., Mouledous L., Zajac J.M., Bihel F., et al. (2012). Involvement of neuropeptide FF receptors in neuroadaptive responses to acute and chronic opiate treatments. Br J Pharmacol $165,424-435$

Elhabazi K., Humbert J.P., Bertin I., Schmitt M., Bihel F., Bourguignon J.J., et al. (2013). Endogenous mammalian RF-amide peptides, including PrRP, kisspeptin and 26RFa, modulate nociception and morphine analgesia via NPFF receptors. Neuropharmacology 75C, 164-171.

Ellacott K.L., Lawrence C.B., Rothwell N.J. \& Luckman S.M. (2002). PRL-releasing peptide interacts with leptin to reduce food intake and body weight. Endocrinology 143, 368-374.

Ellacott K.L., Lawrence C.B., Pritchard L.E. \& Luckman S.M. (2003). Repeated administration of the anorectic factor prolactin-releasing peptide leads to tolerance to its effects on energy homeostasis. Am J Physiol Regul Integr Comp Physiol 285, R1005-1010.

Ellacott K.L., Donald E.L., Clarkson P., Morten J., Masters D., Brennand J., et al. (2005). Characterization of a naturally-occurring polymorphism in the UHR-1 gene encoding the putative rat prolactin-releasing peptide receptor. Peptides 26, 675-681.

Elphick M.R. \& Mirabeau O. (2014). The evolution and variety of RFamide-type neuropeptides: insights from deuterostomian invertebrates. Front Endocrinol (Lausanne) 5, 93.

Elshourbagy N.A., Ames R.S., Fitzgerald L.R., Foley J.J., Chambers J.K., Szekeres P.G., et al. (2000). Receptor for the pain modulatory neuropeptides FF and AF is an orphan $\mathrm{G}$ protein-coupled receptor. J Biol Chem 275 , 25965-25971.

Engstrom M., Brandt A., Wurster S., Savola J.M. \& Panula P. (2003). Prolactin releasing peptide has high affinity and efficacy at neuropeptide FF2 receptors. J Pharmacol Exp Ther 305, 825-832.

Espinoza E., Carrigan M., Thomas S.G., Shaw G. \& Edison A.S. (2000). A statistical view of FMRFamide neuropeptide diversity. Mol Neurobiol 21, 35-56.

Estrada K.M., Clay C.M., Pompolo S., Smith J.T. \& Clarke I.J. (2006). Elevated KiSS-1 expression in the arcuate nucleus prior to the cyclic preovulatory gonadotrophin-releasing hormone/lutenising hormone surge in the ewe suggests a stimulatory role for kisspeptin in oestrogen-positive feedback. $J$ Neuroendocrinol 18, 806-809.

Evans J.J. \& Anderson G.M. (2012). Balancing ovulation and anovulation: integration of the reproductive and energy balance axes by neuropeptides. Hum Reprod Update 18, 313-332.

Fang Q., Guo J., Chang M., Chen L.X., Chen Q. \& Wang R. (2005). Neuropeptide FF receptors exert contractile activity via inhibition of nitric oxide release in the mouse distal colon. Peptides 26, 791-797. 
Fang Q., Wang Y.Q., He F., Guo J., Guo J., Chen Q., et al. (2008). Inhibition of neuropeptide FF (NPFF)induced hypothermia and anti-morphine analgesia by RF9, a new selective NPFF receptors antagonist. Regul Pept 147, 45-51.

Fang P., He B., Shi M., Kong G., Dong X., Zhub Y., et al. (2015). The regulative effect of galanin family members on link of energy metabolism and reproduction. Peptides 71, 240-249.

Feng Y., Zhao H., An X.F., Ma S.L. \& Chen B.Y. (2007). Expression of brain prolactin releasing peptide (PrRP) changes in the estrous cycle of female rats. Neurosci Lett 419, 38-42.

Findeisen M., Rathmann D. \& Beck-Sickinger A.G. (2011a). RF-amide Peptides: Structure, Function, Mechanisms and Pharmaceutical Potential. Pharmaceuticals 4, 1248-1280.

Findeisen M., Rathmann D. \& Beck-Sickinger A.G. (2011b). Structure-activity studies of RF-amide peptides reveal subtype-selective activation of neuropeptide FF1 and FF2 receptors. ChemMedChem 6, 1081-1093.

Findeisen M., Würker C., Rathmann D., Meier R., Meiler J., Olsson R., et al. (2012). Selective Mode of Action of Guanidine-Containing Non-Peptides at Human NPFF Receptors. Journal of Medicinal Chemistry 55, 6124-6136.

Forray C.C., Craig D., Kawakami J., Konkel M.J., Boteju L.W., Wetzel J.M., et al. (2003). Preparation of quinazolinylguanidines, quinolinylguanidines, and N-sulfonyl arginylphenylalaninamides for the treatment of pain, (Corporation, S. P. ed).

Fox S.R. \& Smith M.S. (1985). Changes in the pulsatile pattern of luteinizing hormone secretion during the rat estrous cycle. Endocrinology 116, 1485-1492.

Franceschini I., Lomet D., Cateau M., Delsol G., Tillet Y. \& Caraty A. (2006). Kisspeptin immunoreactive cells of the ovine preoptic area and arcuate nucleus co-express estrogen receptor alpha. Neurosci Lett 401, 225230.

Fredriksson R., Lagerström M.C., Lundin L-G. \& Schiöth H.B. (2003). The G-Protein-Coupled Receptors in the Human Genome Form Five Main Families. Phylogenetic Analysis, Paralogon Groups, and Fingerprints. Mol Pharmacol 63,1256-1272.

Fu L.Y. \& van den Pol A.N. (2010). Kisspeptin directly excites anorexigenic proopiomelanocortin neurons but inhibits orexigenic neuropeptide Y cells by an indirect synaptic mechanism. J Neurosci 30, 10205-10219.

Fujii R., Fukusumi S., Hosoya M., Kawamata Y., Habata Y., Hinuma S., et al. (1999). Tissue distribution of prolactin-releasing peptide (PrRP) and its receptor. Regul Pept 83, 1-10.

Fukusumi S., Habata Y., Yoshida H., Iijima N., Kawamata Y., Hosoya M., et al. (2001). Characteristics and distribution of endogenous RF-amide-related peptide-1. Biochim Biophys Acta 1540, 221-232.

Fukusumi S., Yoshida H., Fujii R., Maruyama M., Komatsu H., Habata Y., et al. (2003). A new peptidic ligand and its receptor regulating adrenal function in rats. J Biol Chem 278, 46387-46395.

Fukusumi S., Fujii R. \& Hinuma S. (2006). Recent advances in mammalian RF-amide peptides: the discovery and functional analyses of PrRP, RFRPs and QRFP. Peptides 27, 1073-1086.

Funes S., Hedrick J.A., Vassileva G., Markowitz L., Abbondanzo S., Golovko A., et al. (2003). The KiSS-1 receptor GPR54 is essential for the development of the murine reproductive system. Biochem Biophys Res Commun 312, 1357-1363.

Galandrin S., Oligny-Longpre G. \& Bouvier M. (2007). The evasive nature of drug efficacy: implications for drug discovery. Trends Pharmacol Sci 28, 423-430.

Galusca B., Jeandel L., Germain N., Alexandre D., Leprince J., Anouar Y., et al. (2012). Orexigenic neuropeptide 26RFa: new evidence for an adaptive profile of appetite regulation in anorexia nervosa. J Clin Endocrinol Metab 97, 2012-2018.

Gao Q. \& Horvath T.L. (2008). Neuronal control of energy homeostasis. FEBS Lett 582, 132-141.

Garcia-Galiano D., van Ingen Schenau D., Leon S., Krajnc-Franken M.A., Manfredi-Lozano M., Romero-Ruiz A., et al. (2012). Kisspeptin signaling is indispensable for neurokinin B, but not glutamate, stimulation of gonadotropin secretion in mice. Endocrinology 153, 316-328.

Garcia-Garcia R.M. (2012). Integrative control of energy balance and reproduction in females. ISRN Vet Sci 2012, 121389.

Gaubert G., Bertozzi F., Kelly N.M., Pawlas J., Scully A.L., Nash N.R., et al. (2009). Discovery of Selective Nonpeptidergic Neuropeptide FF2 Receptor Agonists. Journal of Medicinal Chemistry 52, 6511-6514.

Gaytan F., Gaytan M., Castellano J.M., Romero M., Roa J., Aparicio B., et al. (2009). KiSS-1 in the mammalian ovary: distribution of kisspeptin in human and marmoset and alterations in KiSS-1 mRNA levels in a rat model of ovulatory dysfunction. Am J Physiol Endocrinol Metab 296, E520-531.

Gaytan M., Castellano J.M., Roa J., Sanchez-Criado J.E., Tena-Sempere M. \& Gaytan F. (2007). Expression of KiSS-1 in rat oviduct: possible involvement in prevention of ectopic implantation? Cell Tissue Res 329, 571579.

Gealageas R., Schneider S., Humbert J.-P., Bertin I., Schmitt M., Laboureyras E., et al. (2012). Development of sub-nanomolar dipeptidic ligands of neuropeptide FF receptors. Bioorganic \& Medicinal Chemistry Letters $22,7471-7474$. 
Gelot A., Mazarguil H., Dupuy P., Francés B., Gouardères C., Roumy M., et al. (1998). Biochemical, cellular and pharmacological activities of a human neuropeptide FF-related peptide. Eur J Pharmacol 354, 1671 172.

George J.T., Veldhuis J.D., Roseweir A.K., Newton C.L., Faccenda E., Millar R.P., et al. (2011). Kisspeptin-10 Is a Potent Stimulator of LH and Increases Pulse Frequency in Men. J Clin Endocrinol Metab 96, E1228E1236.

Georgsson J., Bergstrom F., Nordqvist A., Watson M.J., Blundell C.D., Johansson M.J., et al. (2014). GPR103 Antagonists Demonstrating Anorexigenic Activity in Vivo: Design and Development of Pyrrolo 2,3-c pyridines That Mimic the C-Terminal Arg-Phe Motif of QRFP26. Journal of Medicinal Chemistry 57, 59355948.

Gherardi N. \& Zajac J.M. (1997). Neuropeptide FF receptors of mouse olfactory bulb: binding properties and stimulation of adenylate cyclase activity. Peptides 18, 577-583.

Gibson E.M., Humber S.A., Jain S., Williams W.P., 3rd, Zhao S., Bentley G.E., et al. (2008). Alterations in RFamide-related peptide expression are coordinated with the preovulatory luteinizing hormone surge. Endocrinology 149, 4958-4969.

Gicquel S., Mazarguil H., Allard M., Simonnet G. \& Zajac J.M. (1992). Analogues of F8Famide resistant to degradation, with high affinity and in vivo effects. Eur J Pharmacol 222, 61-67.

Gicquel S., Mazarguil H., Desprat C., Allard M., Devillers J.P., Simonnet G., et al. (1994). Structure-activity study of neuropeptide FF: contribution of N-terminal regions to affinity and activity. J Med Chem 37, 34773481 .

Glanowska K.M., Burger L.L. \& Moenter S.M. (2014). Development of Gonadotropin-Releasing Hormone Secretion and Pituitary Response. J Neurosci 34, 15060-15069.

Gogan F., Beattie I.A., Hery M., Laplante E. \& Kordon D. (1980). Effect of neonatal administration of steroids or gonadectomy upon oestradiol-induced luteinizing hormone release in rats of both sexes. J Endocrinol 85, 69-74.

Gogan F., Slama A., Bizzini-Koutznetzova B., Dray F. \& Kordon C. (1981). Importance of perinatal testosterone in sexual differentiation in the male rat. $J$ Endocrinol 91, 75-79.

Goldman B.D. (2001). Mammalian photoperiodic system: formal properties and neuroendocrine mechanisms of photoperiodic time measurement. J Biol Rhythms 16, 283-301.

Goncharuk V., Zeng Z., Wang R., MacTavish D. \& Jhamandas J.H. (2004). Distribution of the neuropeptide FF1 receptor (hFF1) in the human hypothalamus and surrounding basal forebrain structures: immunohistochemical study. J Comp Neurol 474, 487-503.

Goncharuk V. \& Jhamandas J.H. (2008). Neuropeptide FF2 receptor distribution in the human brain. An immunohistochemical study. Peptides 29, 1544-1553.

Goncharuk V.D., Buijs R.M., Mactavish D. \& Jhamandas J.H. (2006). Neuropeptide FF distribution in the human and rat forebrain: a comparative immunohistochemical study. J Comp Neurol 496, 572-593.

Goodman R.L., Lehman M.N., Smith J.T., Coolen L.M., de Oliveira C.V., Jafarzadehshirazi M.R., et al. (2007). Kisspeptin neurons in the arcuate nucleus of the ewe express both dynorphin A and neurokinin B. Endocrinology 148, 5752-5760.

Gottsch M.L., Cunningham M.J., Smith J.T., Popa S.M., Acohido B.V., Crowley W.F., et al. (2004). A role for kisspeptins in the regulation of gonadotropin secretion in the mouse. Endocrinology 145, 4073-4077.

Gouarderes C., Kar S. \& Zajac J-M. (1996). Presence of neuropeptide FF receptors on primary afferent fibres of the rat spinal cord. Neuroscience 74, 21-27.

Gouarderes C., Tafani J.A., Mazarguil H. \& Zajac J.M. (1997). Autoradiographic characterization of rat spinal neuropeptide FF receptors by using [125I][D.Tyr1, (NMe)Phe3]NPFF. Brain Res Bull 42, 231-238.

Gouarderes C., Roumy M., Advokat C., Jhamandas K. \& Zajac J.M. (2000). Dual localization of neuropeptide FF receptors in the rat dorsal horn. Synapse 35, 45-52.

Gouarderes C., Mollereau C., Tafani J.A., Mazarguil H. \& Zajac J.M. (2001). [(125)I]EYF: a new high affinity radioligand to neuropeptide FF receptors. Peptides 22, 623-629.

Gouarderes C., Quelven I., Mollereau C., Mazarguil H., Rice S.Q. \& Zajac J.M. (2002). Quantitative autoradiographic distribution of NPFF1 neuropeptide FF receptor in the rat brain and comparison with NPFF2 receptor by using $\left[{ }^{125} \mathrm{I}\right] \mathrm{YVP}$ and $\left[{ }^{125} \mathrm{I}\right] \mathrm{EYF}$ as selective radioligands. Neuroscience 115, 349-361.

Gouarderes C., Faura C.C. \& Zajac J.M. (2004a). Rodent strain differences in the NPFF1 and NPFF2 receptor distribution and density in the central nervous system. Brain Res 1014, 61-70.

Gouarderes C., Puget A. \& Zajac J.M. (2004b). Detailed distribution of neuropeptide FF receptors (NPFF1 and NPFF2) in the rat, mouse, octodon, rabbit, guinea pig, and marmoset monkey brains: a comparative autoradiographic study. Synapse 51, 249-269.

Gouarderes C., Mazarguil H., Mollereau C., Chartrel N., Leprince J., Vaudry H., et al. (2007). Functional differences between NPFF1 and NPFF2 receptor coupling: high intrinsic activities of RF-amide-related peptides on stimulation of [35S]GTPgammaS binding. Neuropharmacology 52, 376-386. 
Granata R., Settanni F., Trovato L., Gallo D., Gesmundo I., Nano R., et al. (2014). RF-amide peptides 43RFa and 26RFa both promote survival of pancreatic beta-cells and human pancreatic islets but exert opposite effects on insulin secretion. Diabetes 63, 2380-2393.

Greives T.J., Mason A.O., Scotti M.A., Levine J., Ketterson E.D., Kriegsfeld L.J., et al. (2007). Environmental control of kisspeptin: implications for seasonal reproduction. Endocrinology 148, 1158-1166.

Gu W., Geddes B.J., Zhang C., Foley K.P. \& Stricker-Krongrad A. (2004). The prolactin-releasing peptide receptor (GPR10) regulates body weight homeostasis in mice. J Mol Neurosci 22, 93-103.

Gutierrez-Pascual E., Leprince J., Martinez-Fuentes A.J., Segalas-Milazzo I., Pineda R., Roa J., et al. (2009). In Vivo and in Vitro Structure-Activity Relationships and Structural Conformation of Kisspeptin-10-Related Peptides. Molecular Pharmacology 76, 58-67.

Haga Y., Mizutani S. \& Sato N. (2010). Preparation of indole-2-carboxamide derivatives as QRFP receptor (GPR103) antagonists, (Banyu Pharmaceutical Co., L. ed).

Hahn J.D. \& Coen C.W. (2006). Comparative study of the sources of neuronal projections to the site of gonadotrophin-releasing hormone perikarya and to the anteroventral periventricular nucleus in female rats. $J$ Comp Neurol 494, 190-214.

Han S.K., Gottsch M.L., Lee K.J., Popa S.M., Smith J.T., Jakawich S.K., et al. (2005). Activation of gonadotropin-releasing hormone neurons by kisspeptin as a neuroendocrine switch for the onset of puberty. $J$ Neurosci 25, 11349-11356.

Harbid A.A., McLeod B.J., Caraty A. \& Anderson G.M. (2013). Seasonal changes in RF-amide-related peptide3 neurons in the hypothalamus of a seasonally breeding marsupial species, the brushtail possum (Trichosurus vulpecula). J Comp Neurol 521, 3030-3041.

Hastings M.H., Walker A.P., Roberts A.C. \& Herbert J. (1988). Intra-hypothalamic melatonin blocks photoperiodic responsiveness in the male Syrian hamster. Neuroscience 24, 987-991.

Hauge-Evans A.C., Richardson C.C., Milne H.M., Christie M.R., Persaud S.J. \& Jones P.M. (2006). A role for kisspeptin in islet function. Diabetologia 49, 2131-2135.

Hayakawa J., Ohmichi M., Tasaka K., Kanda Y., Adachi K., Nishio Y., et al. (2002). Regulation of the PRL promoter by Akt through cAMP response element binding protein. Endocrinology 143, 13-22.

Herbison A.E., de Tassigny X., Doran J. \& Colledge W.H. (2010). Distribution and postnatal development of Gpr54 gene expression in mouse brain and gonadotropin-releasing hormone neurons. Endocrinology 151, 312-321.

Herrera-Herrera M.L. \& Salazar-Olivo L.A. (2008). RF-amide neuropeptides inhibit murine and human adipose differentiation. Biochem Biophys Res Commun 377, 29-34.

Hinuma S., Habata Y., Fujii R., Kawamata Y., Hosoya M., Fukusumi S., et al. (1998). A prolactin-releasing peptide in the brain. Nature 393, 272-276.

Hinuma S., Shintani Y., Fukusumi S., Iijima N., Matsumoto Y., Hosoya M., et al. (2000). New neuropeptides containing carboxy-terminal RF-amide and their receptor in mammals. Nat Cell Biol 2, 703-708.

Hirschberg A.L. (2012). Sex hormones, appetite and eating behaviour in women. Maturitas 71, 248-256.

Hitchcock S.A. (2008). Blood-brain barrier permeability considerations for CNS-targeted compound library design. Current Opinion in Chemical Biology 12, 318-323.

Hizume T., Watanobe H., Yoneda M., Suda T. \& Schioth H.B. (2000). Involvement of prolactin-releasing peptide in the preovulatory luteinizing hormone and prolactin surges in the rat. Biochem Biophys Res Commun 279, 35-39.

Houben T., Deboer T., van Oosterhout F. \& Meijer J.H. (2009). Correlation with behavioral activity and rest implies circadian regulation by SCN neuronal activity levels. J Biol Rhythms 24, 477-487.

Hrabovszky E., Ciofi P., Vida B., Horvath M.C., Keller E., Caraty A., et al. (2010). The kisspeptin system of the human hypothalamus: sexual dimorphism and relationship with gonadotropin-releasing hormone and neurokinin B neurons. Eur J Neurosci 31, 1984-1998.

Hsu M.C., Wang J.Y., Lee Y.J., Jong D.S., Tsui K.H. \& Chiu C.H. (2014). Kisspeptin modulates fertilization capacity of mouse spermatozoa. Reproduction 147, 835-845.

Huang D.W., Di R., Wang J.X., Chu M.X., He J.N., Cao G.L., et al. (2012). Analysis on DNA sequence of goat RFRP gene and its possible association with average daily sunshine duration. Mol Biol Rep 39, 9167-9177.

Hunt S.C., Hasstedt S.J., Xin Y., Dalley B.K., Milash B.A., Yakobson E., et al. (2011). Polymorphisms in the NPY2R gene show significant associations with BMI that are additive to FTO, MC4R, and NPFFR2 gene effects. Obesity (Silver Spring) 19, 2241-2247.

Ibata Y., Iijima N., Kataoka Y., Kakihara K., Tanaka M., Hosoya M., et al. (2000). Morphological survey of prolactin-releasing peptide and its receptor with special reference to their functional roles in the brain. Neurosci Res 38, 223-230.

Iijima N., Kataoka Y., Kakihara K., Bamba H., Tamada Y., Hayashi S., et al. (1999). Cytochemical study of prolactin-releasing peptide (PrRP) in the rat brain. Neuroreport 10, 1713-1716. 
Irwig M.S., Fraley G.S., Smith J.T., Acohido B.V., Popa S.M., Cunningham M.J., et al. (2004). Kisspeptin activation of gonadotropin releasing hormone neurons and regulation of KiSS-1 mRNA in the male rat. Neuroendocrinology 80, 264-272.

Ishiwata T., Hasegawa H., Yasumatsu M., Akano F., Yazawa T., Otokawa M., et al. (2001). The role of preoptic area and anterior hypothalamus and median raphe nucleus on thermoregulatory system in freely moving rats. Neurosci Lett 306, 126-128.

Janati A., Talbi R., Klosen P., Mikkelsen J.D., Magoul R., Simonneaux V., et al. (2013). Distribution and seasonal variation in hypothalamic RF-amide peptides in a semi-desert rodent, the jerboa. J Neuroendocrinol 25, 402-411.

Jayasena C.N., Nijher G.M., Abbara A., Murphy K.G., Lim A., Patel D., et al. (2010). Twice-weekly administration of kisspeptin-54 for 8 weeks stimulates release of reproductive hormones in women with hypothalamic amenorrhea. Clin Pharmacol Ther 88, 840-847.

Jayasena C.N., Comninos A.N., Veldhuis J.D., Misra S., Abbara A., Izzi-Engbeaya C., et al. (2013). A single injection of kisspeptin-54 temporarily increases luteinizing hormone pulsatility in healthy women. Clinical Endocrinology 79, 558-563.

Jayasena C.N., Abbara A., Veldhuis J.D., Comninos A.N., Ratnasabapathy R., De Silva A., et al. (2014). Increasing LH Pulsatility in Women With Hypothalamic Amenorrhoea Using Intravenous Infusion of Kisspeptin-54. J Clin Endocrinol Metab 99, E953-E961.

Jékely G. (2013). Global view of the evolution and diversity of metazoan neuropeptide signalling. Proc Natl Acad Sci U S A 110, 8702-8707.

Jhamandas J.H., Jhamandas A. \& Harris K.H. (2001). New central projections of neuropeptide FF: colateral branching pathways in the brainstem and hypothalamus in the rat. J Chem Neuroanat 21, 171-179.

Jhamandas J.H. \& MacTavish D. (2003). Central administration of neuropeptide FF causes activation of oxytocin paraventricular hypothalamic neurones that project to the brainstem. J Neuroendocrinol 15, 24-32.

Jhamandas K., Milne B., Sutak M., Gouarderes C., Zajac J.M. \& Yang H.Y. (2006). Facilitation of spinal morphine analgesia in normal and morphine tolerant animals by neuropeptide SF and related peptides. Peptides 27, 953-963.

Ji K., Ye L., Mason M.D. \& Jiang W.G. (2013). The Kiss-1/Kiss-1R complex as a negative regulator of cell motility and cancer metastasis (Review). Int J Mol Med 32, 747-754.

Jiang Y., Luo L., Gustafson E.L., Yadav D., Laverty M., Murgolo N., et al. (2003). Identification and characterization of a novel RF-amide peptide ligand for orphan G-protein-coupled receptor SP9155. J Biol Chem 278, 27652-27657.

Johnson M.A., Tsutsui K. \& Fraley G.S. (2007). Rat RF-amide-related peptide-3 stimulates GH secretion, inhibits LH secretion, and has variable effects on sex behavior in the adult male rat. Horm Behav 51, 171180.

Johnson M.A. \& Fraley G.S. (2008). Rat RFRP-3 alters hypothalamic GHRH expression and growth hormone secretion but does not affect KiSS-1 gene expression or the onset of puberty in male rats. Neuroendocrinology 88, 305-315.

Johnston J.D., Tournier B.B., Andersson H., Masson-Pevet M., Lincoln G.A. \& Hazlerigg D.G. (2006). Multiple effects of melatonin on rhythmic clock gene expression in the mammalian pars tuberalis. Endocrinology 147 , 959-965.

Jones P.B. \& Hsueh A.J. (1981). Direct effects of gonadotropin releasing hormone and its antagonist upon ovarian functions stimulated by FSH, prolactin, ans LH. Biol Reprod 24, 747-759.

Jorgensen S.R., Andersen M.D., Overgaard A. \& Mikkelsen J.D. (2014). Changes in RF-amide-related peptide-1 (RFRP-1)-immunoreactivity during postnatal development and the estrous cycle. Endocrinology 155, 44024410.

Jossart C., Mulumba M., Granata R., Gallo D., Ghigo E., Marleau S., et al. (2014). Pyroglutamylated RF-amide peptide (QRFP) gene is regulated by metabolic endotoxemia. Mol Endocrinol 28, 65-79.

Journigan V.B., Mésangeau C., Vyas N., Eans S.O., Cutler S.J., McLaughlin J.P., et al. (2014). Nonpeptide Small Molecule Agonist and Antagonist Original Leads for Neuropeptide FF1 and FF2 Receptors. Journal of Medicinal Chemistry.

Kadokawa H., Matsui M., Hayashi K., Matsunaga N., Kawashima C., Shimizu T., et al. (2008). Peripheral administration of kisspeptin-10 increases plasma concentrations of GH as well as LH in prepubertal Holstein heifers. J Endocrinol 196, 331-334.

Kadokawa H., Shibata M., Tanaka Y., Kojima T., Matsumoto K., Oshima K., et al. (2009). Bovine C-terminal octapeptide of RF-amide-related peptide-3 suppresses luteinizing hormone (LH) secretion from the pituitary as well as pulsatile LH secretion in bovines. Domest Anim Endocrinol 36, 219-224.

Kaewwongse M., Takayanagi Y. \& Onaka T. (2011). Effects of RF-amide-related peptide (RFRP)-1 and RFRP3 on oxytocin release and anxiety-related behaviour in rats. J Neuroendocrinol 23, 20-27. 
Kalamatianos T., Grimshaw S.E., Poorun R., Hahn J.D. \& Coen C.W. (2008). Fasting reduces KiSS-1 expression in the anteroventral periventricular nucleus (AVPV): effects of fasting on the expression of KiSS1 and neuropeptide Y in the AVPV or arcuate nucleus of female rats. J Neuroendocrinol 20, 1089-1097.

Kalliomaki M.L. \& Panula P. (2004). Neuropeptide FF, but not prolactin-releasing peptide, mRNA is differentially regulated in the hypothalamic and medullary neurons after salt loading. Neuroscience 124, 8187.

Kalliomaki M.L., Pertovaara A., Brandt A., Wei H., Pietila P., Kalmari J., et al. (2004). Prolactin-releasing peptide affects pain, allodynia and autonomic reflexes through medullary mechanisms. Neuropharmacology 46, 412-424.

Kampe J., Wiedmer P., Pfluger P.T., Castaneda T.R., Burget L., Mondala H., et al. (2006). Effect of central administration of QRFP(26) peptide on energy balance and characterization of a second QRFP receptor in rat. Brain Res 1119, 133-149.

Karsch F.J., Dierschke D.K., Weick R.F., Yamaji T., Hotchkiss J. \& Knobil E. (1973). Positive and negative feedback control by estrogen of luteinizing hormone secretion in the rhesus monkey. Endocrinology 92, 799804.

Karsch F.J., Bittman E.L., Foster D.L., Goodman R.L., Legan S.J. \& Robinson J.E. (1984). Neuroendocrine basis of seasonal reproduction. Recent Prog Horm Res 40, 185-232.

Kataoka Y., Iijima N., Yano T., Kakihara K., Hayashi S., Hinuma S., et al. (2001). Gonadal regulation of PrRP mRNA expression in the nucleus tractus solitarius and ventral and lateral reticular nuclei of the rat. Brain Res Mol Brain Res 87, 42-47.

Kauffman A.S., Gottsch M.L., Roa J., Byquist A.C., Crown A., Clifton D.K., et al. (2007a). Sexual differentiation of Kiss1 gene expression in the brain of the rat. Endocrinology 148, 1774-1783.

Kauffman A.S., Park J.H., McPhie-Lalmansingh A.A., Gottsch M.L., Bodo C., Hohmann J.G., et al. (2007b). The kisspeptin receptor GPR54 is required for sexual differentiation of the brain and behavior. J Neurosci 27, 8826-8835.

Kavaliers M. \& Hirst M. (1986). FMRF-amide: an endogenous peptide with marked inhibitory effects on opioidinduced feeding behavior. Brain Res Bull 17, 403-408.

Kawamata Y., Fujii R., Fukusumi S., Habata Y., Hosoya M., Hinuma S., et al. (2000). Analyses for susceptibility of rat anterior pituitary cells to prolactin-releasing peptide. Endocrine 12, 215-221.

Keen K.L., Wegner F.H., Bloom S.R., Ghatei M.A. \& Terasawa E. (2008). An increase in kisspeptin-54 release occurs with the pubertal increase in luteinizing hormone-releasing hormone-1 release in the stalk-median eminence of female rhesus monkeys in vivo. Endocrinology 149, 4151-4157.

Kenakin T. \& Onaran O. (2002). The ligand paradox between affinity and efficacy: can you be there and not make a difference? Trends Pharmacol Sci 23, 275-280.

Kenakin T. \& Christopoulos A. (2013). Signalling bias in new drug discovery: detection, quantification and therapeutic impact. Nat Rev Drug Discov 12, 205-216.

Kersante F., Mollereau C., Zajac J.M. \& Roumy M. (2006). Anti-opioid activities of NPFF1 receptors in a SHSY5Y model. Peptides 27, 980-989.

Kim D.K., Yun S., Son G.H., Hwang J.I., Park C.R., Kim J.I., et al. (2014). Coevolution of the spexin/galanin/kisspeptin family: spexin activates galanin receptor type II and III. Endocrinology 155, 18641873.

Kim J., Semaan S.J., Clifton D.K., Steiner R.A., Dhamija S. \& Kauffman A.S. (2011). Regulation of Kiss1 expression by sex steroids in the amygdala of the rat and mouse. Endocrinology 152, 2020-2030.

Kim J.S., Brownjohn P.W., Dyer B.S., Beltramo M., Walker C.S., Hay D.L., et al. (2015). Anxiogenic and Stressor Effects of the Hypothalamic Neuropeptide RFRP-3 Are Overcome by the NPFFR Antagonist GJ14. Endocrinology 156, 4152-4162.

Kimura A., Ohmichi M., Tasaka K., Kanda Y., Ikegami H., Hayakawa J., et al. (2000). Prolactin-releasing peptide activation of the prolactin promoter is differentially mediated by extracellular signal-regulated protein kinase and c-Jun N-terminal protein kinase. J Biol Chem 275, 3667-3674.

Kinoshita M., Tsukamura H., Adachi S., Matsui H., Uenoyama Y., Iwata K., et al. (2005). Involvement of central metastin in the regulation of preovulatory luteinizing hormone surge and estrous cyclicity in female rats. Endocrinology 146, 4431-4436.

Kirby H.R., Maguire J.J., Colledge W.H. \& Davenport A.P. (2010). International Union of Basic and Clinical Pharmacology. LXXVII. Kisspeptin receptor nomenclature, distribution, and function. Pharmacol Rev 62, 565-578.

Kivipelto L., Majane E.A., Yang H.Y. \& Panula P. (1989). Immunohistochemical distribution and partial characterization of FLFQPQRF-amidelike peptides in the central nervous system of rats. J Comp Neurol 286, 269-287.

Kivipelto L. \& Panula P. (1991). Central neuronal pathways containing FLFQPQRF-amide-like (morphinemodulating) peptides in the rat brain. Neuroscience 41, 137-148. 
Klingerman C.M., Williams W.P., 3rd, Simberlund J., Brahme N., Prasad A., Schneider J.E., et al. (2011). Food Restriction-Induced Changes in Gonadotropin-Inhibiting Hormone Cells are Associated with Changes in Sexual Motivation and Food Hoarding, but not Sexual Performance and Food Intake. Front Endocrinol (Lausanne) 2, 101.

Klosen P., Bienvenu C., Demarteau O., Dardente H., Guerrero H., Pevet P., et al. (2002). The mt1 melatonin receptor and RORbeta receptor are co-localized in specific TSH-immunoreactive cells in the pars tuberalis of the rat pituitary. J Histochem Cytochem 50, 1647-1657.

Knobil E. (1974). On the control of gonadotropin secretion in the rhesus monkey. Recent Prog Horm Res 30, 146.

Kobayashi T., Sasaki S., Tomita N., Fukui S., Kuroda N., Nakayama M., et al. (2010a). Synthesis and structureactivity relationships of 2-acylamino-4,6-diphenylpyridine derivatives as novel antagonists of GPR54. Bioorganic \& Medicinal Chemistry 18, 3841-3859.

Kobayashi T., Sasaki S., Tomita N., Fukui S., Nakayama M., Kiba A., et al. (2010b). 2-Acylamino-4,6diphenylpyridine derivatives as novel GPR54 antagonists with good brain exposure and in vivo efficacy for plasma LH level in male rats. Bioorganic \& Medicinal Chemistry 18, 5157-5171.

Kotani M., Detheux M., Vandenbogaerde A., Communi D., Vanderwinden J.M., Le Poul E., et al. (2001a). The metastasis suppressor gene KiSS-1 encodes kisspeptins, the natural ligands of the orphan G protein-coupled receptor GPR54. J Biol Chem 276, 34631-34636.

Kotani M., Mollereau C., Detheux M., Le Poul E., Brézillon S., Vakili J., et al. (2001b). Functional characterization of a human receptor for neuropeptide FF and related peptides.. Br J Pharmacol 133, 138144.

Kovacs A., Laszlo K., Galosi R., Toth K., Ollmann T., Peczely L., et al. (2012). Microinjection of RFRP-1 in the central nucleus of amygdala decreases food intake in the rat. Brain Res Bull 88, 589-595.

Kovacs A., Laszlo K., Galosi R., Ollmann T., Peczely L., Zagoracz O., et al. (2014). Intraamygdaloid microinjection of RF-amide-related peptide-3 decreases food intake in rats. Brain Res Bull 107, 61-68.

Krajniak K.G. (2013). Invertebrate FMRFamide Related Peptides. Protein \& Peptide Letters 20, 647-670.

Kriegsfeld L.J., Mei D.F., Bentley G.E., Ubuka T., Mason A.O., Inoue K., et al. (2006). Identification and characterization of a gonadotropin-inhibitory system in the brains of mammals. Proc Natl Acad Sci U S A $103,2410-2415$.

Laemmle B., Schindler M., Beilmann M., Hamilton B.S., Doods H.N. \& Wieland H.A. (2003). Characterization of the NPGP receptor and identification of a novel short mRNA isoform in human hypothalamus. Regul Pept $111,21-29$.

Lafontan M. \& Berlan M. (1993). Fat cell adrenergic receptors and the control of white and brown fat cell function. J Lipid Res 34, 1057-1091.

Lagrange A.H., Rønnekleiv O.K. \& Kelly M.J. (1995). Estradiol-17 beta and mu-opioid peptides rapidly hyperpolarize GnRH neurons: a cellular mechanism of negative feedback? Endocrinology 136, 2341-2344.

Lanfranco F., Gromoll J., von Eckardstein S., Herding E.M., Nieschlag E. \& Simoni M. (2005). Role of sequence variations of the $\mathrm{GnRH}$ receptor and $\mathrm{G}$ protein-coupled receptor 54 gene in male idiopathic hypogonadotropic hypogonadism. Eur J Endocrinol 153, 845-852.

Lang R., Gundlach A.L., Holmes F.E., Hobson S.A., Wynick D., Hökfelt T., et al. (2015). Physiology, Signaling, and Pharmacology of Galanin Peptides and Receptors: Three Decades of Emerging Diversity. Pharmacol Rev 67, 118-175.

Langmead C.J., Szekeres P.G., Chambers J.K., Ratcliffe S.J., Jones D.N., Hirst W.D., et al. (2000). Characterization of the binding of [(125)I]-human prolactin releasing peptide (PrRP) to GPR10, a novel G protein coupled receptor. Br J Pharmacol 131, 683-688.

Lapatto R., Pallais J.C., Zhang D., Chan Y.M., Mahan A., Cerrato F., et al. (2007). Kiss1-/- mice exhibit more variable hypogonadism than Gpr54-/- mice. Endocrinology 148, 4927-4936.

Laurent P., Becker J.A., Valverde O., Ledent C., de Kerchove d'Exaerde A., Schiffmann S.N., et al. (2005). The prolactin-releasing peptide antagonizes the opioid system through its receptor GPR10. Nat Neurosci 8, 17351741.

Lawrence C.B., Celsi F., Brennand J. \& Luckman S.M. (2000). Alternative role for prolactin-releasing peptide in the regulation of food intake. Nat Neurosci 3, 645-646.

Lawrence C.B., Ellacott K.L. \& Luckman S.M. (2002). PRL-releasing peptide reduces food intake and may mediate satiety signaling. Endocrinology 143, 360-367.

Lawrence C.B., Liu Y.L., Stock M.J. \& Luckman S.M. (2004). Anorectic actions of prolactin-releasing peptide are mediated by corticotropin-releasing hormone receptors. Am J Physiol Regul Integr Comp Physiol 286, R101-107.

Le Marec O., Neveu C., Lefranc B., Dubessy C., Boutin J.A., Do-Rego J.C., et al. (2011). Structure-Activity Relationships of a Series of Analogues of the RF-amide-Related Peptide 26RFa. Journal of Medicinal Chemistry 54, 4806-4814. 
Lectez B., Jeandel L., El-Yamani F.Z., Arthaud S., Alexandre D., Mardargent A., et al. (2009). The orexigenic activity of the hypothalamic neuropeptide $26 \mathrm{RFa}$ is mediated by the neuropeptide $\mathrm{Y}$ and proopiomelanocortin neurons of the arcuate nucleus. Endocrinology 150, 2342-2350.

Lee C.H., Wasowicz K., Brown R., Majane E.A., Yang H.T. \& Panula P. (1993). Distribution and characterization of neuropeptide FF-like immunoreactivity in the rat nervous system with a monoclonal antibody. Eur J Neurosci 5, 1339-1348.

Lee D.K., Nguyen T., O'Neill G.P., Cheng R., Liu Y., Howard A.D., et al. (1999). Discovery of a receptor related to the galanin receptors. FEBS Lett 446, 103-107.

Lee D.K., Nguyen T., Lynch K.R., Cheng R., Vanti W.B., Arkhitko O., et al. (2001). Discovery and mapping of ten novel $\mathrm{G}$ protein-coupled receptor genes. Gene 275, 83-91.

Lee J.H., Miele M.E., Hicks D.J., Phillips K.K., Trent J.M., Weissman B.E., et al. (1996). KiSS-1, a novel human malignant melanoma metastasis-suppressor gene. J Natl Cancer Inst 88, 1731-1737.

Lee Y., Yang S.P., Soares M.J. \& Voogt J.L. (2000). Distribution of prolactin-releasing peptide mRNA in the rat brain. Brain Res Bull 51, 171-176.

Lefrere I., De Coppet P., Camelin J.C., Le Lay S., Mercier N., Elshourbagy N., et al. (2002). Neuropeptide AF and FF modulation of adipocyte metabolism. Primary insights from functional genomics and effects on betaadrenergic responsiveness. J Biol Chem 277, 39169-39178.

Legagneux K., Bernard-Franchi G., Poncet F., La Roche A., Colard C., Fellmann D., et al. (2009). Distribution and genesis of the RFRP-producing neurons in the rat brain: comparison with melanin-concentrating hormone- and hypocretin-containing neurons. Neuropeptides 43, 13-19.

Lehman M.N., Merkley C.M., Coolen L.M. \& Goodman R.L. (2010). Anatomy of the kisspeptin neural network in mammals. Brain Res 1364, 90-102.

Lenard N.R. \& Berthoud H.R. (2008). Central and peripheral regulation of food intake and physical activity: pathways and genes. Obesity (Silver Spring) 16 Suppl 3, S11-22.

Leon S., Garcia-Galiano D., Ruiz-Pino F., Barroso A., Manfredi-Lozano M., Romero-Ruiz A., et al. (2014). Physiological roles of gonadotropin-inhibitory hormone signaling in the control of mammalian reproductive axis: studies in the NPFF1 receptor null mouse. Endocrinology 155, 2953-2965.

Lewis D., Freeman D.A., Dark J., Wynne-Edwards K.E. \& Zucker I. (2002). Photoperiodic control of oestrous cycles in Syrian hamsters: mediation by the mediobasal hypothalamus. J Neuroendocrinol 14, 294-299.

Li X., Su J., Lei Z., Zhao Y., Jin M., Fang R., et al. (2012). Gonadotropin-inhibitory hormone (GnIH) and its receptor in the female pig: cDNA cloning, expression in tissues and expression pattern in the reproductive axis during the estrous cycle. Peptides 36, 176-185.

Li X., Su J., Fang R., Zheng L., Lei R., Wang X., et al. (2013). The effects of RFRP-3, the mammalian ortholog of $\mathrm{GnIH}$, on the female pig reproductive axis in vitro. Mol Cell Endocrinol 372, 65-72.

Li X.F., Kinsey-Jones J.S., Cheng Y., Knox A.M., Lin Y., Petrou N.A., et al. (2009). Kisspeptin signalling in the hypothalamic arcuate nucleus regulates GnRH pulse generator frequency in the rat. PLoS One 4, e8334.

Lin S.H., Arai A.C., Wang Z., Nothacker H.P. \& Civelli O. (2001). The carboxyl terminus of the prolactinreleasing peptide receptor interacts with PDZ domain proteins involved in alpha-amino-3-hydroxy-5methylisoxazole-4-propionic acid receptor clustering. Mol Pharmacol 60, 916-923.

Lin S.H., Leslie F.M. \& Civelli O. (2002). Neurochemical properties of the prolactin releasing peptide (PrRP) receptor expressing neurons: evidence for a role of PrRP as a regulator of stress and nociception. Brain Res 952, $15-30$.

Lin S.H. (2008). Prolactin-releasing peptide. Results Probl Cell Differ 46, 57-88.

Liu Q., Guan X.M., Martin W.J., McDonald T.P., Clements M.K., Jiang Q., et al. (2001). Identification and characterization of novel mammalian neuropeptide ff- like peptides that attenuate morphine-induced antinociception. J Biol Chem 276, 36961-36969.

Liu X., Lee K. \& Herbison A.E. (2008). Kisspeptin Excites Gonadotropin-Releasing Hormone Neurons through a Phospholipase C/Calcium-Dependent Pathway Regulating Multiple Ion Channels. Endocrinology 149, $4605-4614$.

Liu X. \& Herbison A.E. (2014). RF9 Excitation of GnRH Neurons Is Dependent Upon Kiss1r in the Adult Male and Female Mouse. Endocrinology 155, 4915-4924.

Lombard M.C., Weil-Fugazza J., Ries C. \& Allard M. (1999). Unilateral joint inflammation induces bilateral and time-dependent changes in neuropeptide FF binding in the superficial dorsal horn of the rat spinal cord: implication of supraspinal descending systems. Brain Res 816, 598-608.

Loos R.J., Lindgren C.M., Li S., Wheeler E., Zhao J.H., Prokopenko I., et al. (2008). Common variants near MC4R are associated with fat mass, weight and risk of obesity. Nat Genet 40, 768-775.

Loret de Mola J.R. (2009). Obesity and its relationship to infertility in men and women. Obstet Gynecol Clin North Am 36, 333-346, ix. 
Luque R.M., Kineman R.D. \& Tena-Sempere M. (2007). Regulation of hypothalamic expression of KiSS-1 and GPR54 genes by metabolic factors: analyses using mouse models and a cell line. Endocrinology 148, 46014611.

Luque R.M., Cordoba-Chacon J., Gahete M.D., Navarro V.M., Tena-Sempere M., Kineman R.D., et al. (2011). Kisspeptin regulates gonadotroph and somatotroph function in nonhuman primate pituitary via common and distinct signaling mechanisms. Endocrinology 152, 957-966.

Lyon A.M. \& Tesmer J.G. (2013). Structural Insights into Phospholipase C- $\beta$ Function. Mol Pharmacol 84, 488500.

Lyubimov Y., Engstrom M., Wurster S., Savola J.M., Korpi E.R. \& Panula P. (2010). Human kisspeptins activate neuropeptide FF2 receptor. Neuroscience 170, 117-122.

Ma L., MacTavish D., Simonin F., Bourguignon J.J., Watanabe T. \& Jhamandas J.H. (2009). Prolactin-releasing peptide effects in the rat brain are mediated through the Neuropeptide FF receptor. Eur J Neurosci 30, 15851593.

MacLean D.B., Matsui H., Suri A., Neuwirth R. \& Colombel M. (2014). Sustained Exposure to the Investigational Kisspeptin Analog, TAK-448, Down-Regulates Testosterone into the Castration Range in Healthy Males and in Patients With Prostate Cancer: Results From Two Phase 1 Studies. J Clin Endocrinol Metab 99, E1445-E1453.

Madsen K.L., Thorsen T.S., Rahbek-Clemmensen T., Eriksen J. \& Gether U. (2012). Protein interacting with C kinase 1 (PICK1) reduces reinsertion rates of interaction partners sorted to Rab11-dependent slow recycling pathway. J Biol Chem 287, 12293-12308.

Maixnerova J., Spolcova A., Pychova M., Blechova M., Elbert T., Rezacova M., et al. (2011). Characterization of prolactin-releasing peptide: binding, signaling and hormone secretion in rodent pituitary cell lines endogenously expressing its receptor. Peptides 32, 811-817.

Majane E.A., Casanova M.F. \& Yang H.Y. (1988). Biochemical characterization of FMRF-NH2-like peptides in spinal cords of various mammalian species using specific radioimmunoassays. Peptides 9, 1137-1144.

Majane E.A. \& Yang H.Y. (1991). Mammalian FMRF-NH2-like peptide in rat pituitary: decrease by osmotic stimulus. Peptides 12, 1303-1308.

Makri A., Pissimissis N., Lembessis P., Polychronakos C. \& Koutsilieris M. (2008). The kisspeptin (KiSS1)/GPR54 system in cancer biology. Cancer Treat Rev 34, 682-692.

Maletinska L., Spolcova A., Maixnerova J., Blechova M. \& Zelezna B. (2011). Biological properties of prolactin-releasing peptide analogs with a modified aromatic ring of a $\mathrm{C}$-terminal phenylalanine amide. Peptides 32, 1887-1892.

Malin D.H., Lake J.R., Fowler D.E., Hammond M.V., Brown S.L., Leyva J.E., et al. (1990). FMRF-NH2-like mammalian peptide precipitates opiate-withdrawal syndrome in the rat. Peptides 11, 277-280.

Malpaux B., Daveau A., Maurice-Mandon F., Duarte G. \& Chemineau P. (1998). Evidence that melatonin acts in the premammillary hypothalamic area to control reproduction in the ewe: presence of binding sites and stimulation of luteinizing hormone secretion by in situ microimplant delivery. Endocrinology 139, 15081516.

Maniscalco J.W., Kreisler A.D. \& Rinaman L. (2012). Satiation and stress-induced hypophagia: examining the role of hindbrain neurons expressing prolactin-releasing Peptide or glucagon-like Peptide 1. Front Neurosci 6, 199.

Marchese A., Heiber M., Nguyen T., Heng H.H., Saldivia V.R., Cheng R., et al. (1995). Cloning and chromosomal mapping of three novel genes, GPR9, GPR10, and GPR14, encoding receptors related to interleukin 8, neuropeptide Y, and somatostatin receptors. Genomics 29, 335-344.

Marot D., Bieche I., Aumas C., Esselin S., Bouquet C., Vacher S., et al. (2007). High tumoral levels of Kiss1 and G-protein-coupled receptor 54 expression are correlated with poor prognosis of estrogen receptor-positive breast tumors. Endocr Relat Cancer 14, 691-702.

Maruyama M., Matsumoto H., Fujiwara K., Kitada C., Hinuma S., Onda H., et al. (1999). Immunocytochemical localization of prolactin-releasing peptide in the rat brain. Endocrinology 140, 2326-2333.

Maruyama M., Matsumoto H., Fujiwara K., Noguchi J., Kitada C., Fujino M., et al. (2001). Prolactin-releasing peptide as a novel stress mediator in the central nervous system. Endocrinology 142, 2032-2038.

Mason A.O., Greives T.J., Scotti M.A., Levine J., Frommeyer S., Ketterson E.D., et al. (2007). Suppression of kisspeptin expression and gonadotropic axis sensitivity following exposure to inhibitory day lengths in female Siberian hamsters. Horm Behav 52, 492-498.

Mason A.O., Duffy S., Zhao S., Ubuka T., Bentley G.E., Tsutsui K., et al. (2010). Photoperiod and reproductive condition are associated with changes in RF-amide-related peptide (RFRP) expression in Syrian hamsters (Mesocricetus auratus). J Biol Rhythms 25, 176-185.

Masson-Pevet M. \& Gauer F. (1994). Seasonality and melatonin receptors in the pars tuberalis in some long day breeders. Biol Signals 3, 63-70. 
Masson-Pevet M., George D., Kalsbeek A., Saboureau M., Lakhdar-Ghazal N. \& Pevet P. (1994). An attempt to correlate brain areas containing melatonin-binding sites with rhythmic functions: a study in five hibernator species. Cell Tissue Res 278, 97-106.

Matsui H., Takatsu Y., Kumano S., Matsumoto H. \& Ohtaki T. (2004). Peripheral administration of metastin induces marked gonadotropin release and ovulation in the rat. Biochem Biophys Res Commun 320, 383-388.

Matsui H., Tanaka A., Yokoyama K., Takatsu Y., Ishikawa K., Asami T., et al. (2012). Chronic Administration of the Metastin/Kisspeptin Analog KISS1-305 or the Investigational Agent TAK-448 Suppresses Hypothalamic Pituitary Gonadal Function and Depletes Plasma Testosterone in Adult Male Rats. Endocrinology 153, 5297-5308.

Matsumoto H., Murakami Y., Horikoshi Y., Noguchi J., Habata Y., Kitada C., et al. (1999). Distribution and characterization of immunoreactive prolactin-releasing peptide (PrRP) in rat tissue and plasma. Biochem Biophys Res Commun 257, 264-268.

Matsuo H., Baba Y., Nair R.M., Arimura A. \& Schally A.V. (1971). Structure of the porcine LH- and FSHreleasing hormone. I. The proposed amino acid sequence. Biochem Biophys Res Commun 43, 1334-1339.

Matsuzaki T., Iwasa T., Kinouchi R., Yoshida S., Murakami M., Gereltsetseg G., et al. (2011). Fasting reduces the kiss1 mRNA levels in the caudal hypothalamus of gonadally intact adult female rats. Endocr J 58, 10031012.

Maurice P., Guillaume J.L., Benleulmi-Chaachoua A., Daulat A.M., Kamal M. \& Jockers R. (2011). GPCRinteracting proteins, major players of GPCR function. Adv Pharmacol 62, 349-380.

Maywood E.S. \& Hastings M.H. (1995). Lesions of the iodomelatonin-binding sites of the mediobasal hypothalamus spare the lactotropic, but block the gonadotropic response of male Syrian hamsters to short photoperiod and to melatonin. Endocrinology 136, 144-53.

Maywood E.S., Bittman E.L. \& Hastings M.H. (1996). Lesions of the melatonin- and androgen-responsive tissue of the dorsomedial nucleus of the hypothalamus block the gonadal response of male Syrian hamsters to programmed infusions of melatonin. Biol Reprod 54, 470-477.

Mazarguil H., Gouarderes C., Tafani J.A.M., Marcus D., Kotani M., Mollereau C., et al. (2001). Structureactivity relationships of neuropeptide FF: role of C-terminal regions. Peptides 22, 1471-1478.

Mazarguil H., Mollereau C., Czaplicki G. \& Zajac J.M. (2012). Study of the N-terminal part of peptidic selective NPFF2 agonists. Peptides 37, 157-160.

Mead E.J., Maguire J.J., Kuc R.E. \& Davenport A.P. (2007). Kisspeptins are novel potent vasoconstrictors in humans, with a discrete localization of their receptor, $\mathrm{G}$ protein-coupled receptor 54 , to atherosclerosis-prone vessels. Endocrinology 148, 140-147.

Mei H., Doran J., Kyle V., Yeo S-H. \& Colledge W.H. (2013). Does kisspeptin signaling have a role in the testes?. Front Endocrinol (Lausanne) 4, 198.

Mera T., Fujihara H., Kawasaki M., Hashimoto H., Saito T., Shibata M., et al. (2006). Prolactin-releasing peptide is a potent mediator of stress responses in the brain through the hypothalamic paraventricular nucleus. Neuroscience 141, 1069-1086.

Messager S., Chatzidaki E.E., Ma D., Hendrick A.G., Zahn D., Dixon J., et al. (2005). Kisspeptin directly stimulates gonadotropin-releasing hormone release via $\mathrm{G}$ protein-coupled receptor 54. Proc Natl Acad Sci U $S$ A 102, 1761-1766.

Meye F.J. \& Adan R.A. (2014). Feelings about food: the ventral tegmental area in food reward and emotional eating. Trends Pharmacol Sci 35, 31-40.

Mi W.L., Mao-Ying Q.L., Liu Q., Wang X.W., Li X., Wang Y.Q., et al. (2009). The distribution of kisspeptin and its receptor GPR54 in rat dorsal root ganglion and up-regulation of its expression after CFA injection. Brain Res Bull 78, 254-260.

Mikkelsen J.D. \& Simonneaux V. (2009). The neuroanatomy of the kisspeptin system in the mammalian brain. Peptides 30, 26-33.

Min L., Leon S., Li H., Pinilla L., Carroll R.S., Tena-Sempere M., et al. (2015). RF9 Acts as a KISS1R Agonist In Vivo and In Vitro. Endocrinology 156, 4639 ï 4648.

Moenter S.M., DeFazio A.R., Pitts G.R. \& Nunemaker C.S. (2003). Mechanisms underlying episodic gonadotropin-releasing hormone secretion. Front Neuroendocrinol 24, 79-93.

Mollereau C., Gouarderes C., Dumont Y., Kotani M., Detheux M., Doods H., et al. (2001). Agonist and antagonist activities on human NPFF(2) receptors of the NPY ligands GR231118 and BIBP3226. Br $J$ Pharmacol 133, 1-4.

Mollereau C., Mazarguil H., Marcus D., Quelven I., Kotani M., Lannoy V., et al. (2002). Pharmacological characterization of human $\mathrm{NPFF}(1)$ and $\mathrm{NPFF}(2)$ receptors expressed in CHO cells by using NPY Y(1) receptor antagonists. Eur J Pharmacol 451, 245-256.

Mollereau C., Mazarguil H., Zajac J.M. \& Roumy M. (2005a). Neuropeptide FF (NPFF) analogs functionally antagonize opioid activities in NPFF2 receptor-transfected SH-SY5Y neuroblastoma cells. Mol Pharmacol 67, 965-975. 
Mollereau C., Roumy M. \& Zajac J-M. (2005b). Opioid-modulating Peptides: Mechanisms of Action. Current Topics in Medicinal Chemistry 5, 341-355.

Mollereau C., Zajac J-M . \& Roumy M. (2007). Staurosporine differentiation of NPFF2 receptor-transfected SHSY5Y neuroblastoma cells induces selectivity of NPFF activity towards opioid receptors. Peptides 28, 11251128.

Molnar C.S., Kallo I., Liposits Z. \& Hrabovszky E. (2011). Estradiol down-regulates RF-amide-related peptide (RFRP) expression in the mouse hypothalamus. Endocrinology 152, 1684-1690.

Moriya R., Sano H., Umeda T., Ito M., Takahashi Y., Matsuda M., et al. (2006). RF-amide peptide QRFP43 causes obesity with hyperphagia and reduced thermogenesis in mice. Endocrinology 147, 2916-2922.

Morley J.E., Levine A.S., Yim G.K. \& Lowy M.T. (1983). Opioid modulation of appetite. Neurosci Biobehav $\operatorname{Rev} 7,281-305$.

Morton G.J., Meek T.H. \& Schwartz M.W. (2014). Neurobiology of food intake in health and disease. Nat Rev Neurosci 15, 367-378.

Mouledous L., Barthas F. \& Zajac J.M. (2010a). Opposite control of body temperature by NPFF1 and NPFF2 receptors in mice. Neuropeptides 44, 453-456.

Mouledous L., Mollereau C. \& Zajac J.M. (2010b). Opioid-modulating properties of the neuropeptide FF system. Biofactors 36, 423-429.

Mounzih K., Lu R. \& Chehab F.F. (1997). Leptin treatment rescues the sterility of genetically obese ob/ob males. Endocrinology 138, 1190-1193.

Muir A.I., Chamberlain L., Elshourbagy N.A., Michalovich D., Moore D.J., Calamari A., et al. (2001). AXOR12, a novel human G protein-coupled receptor, activated by the peptide KiSS-1. J Biol Chem 276, 28969-28975.

Mulumba M., Jossart C., Granata R., Gallo D., Escher E., Ghigo E., et al. (2010). GPR103b functions in the peripheral regulation of adipogenesis. Mol Endocrinol 24, 1615-1625.

Murakami M., Matsuzaki T., Iwasa T., Yasui T., Irahara M., Osugi T., et al. (2008). Hypophysiotropic role of RF-amide-related peptide-3 in the inhibition of LH secretion in female rats. J Endocrinol 199, 105-112.

Murase T., Arima H., Kondo K. \& Oiso Y. (1996). Neuropeptide FF reduces food intake in rats. Peptides 17, 353-354.

Nanmoku T., Takekoshi K., Isobe K., Kawakami Y., Nakai T. \& Okuda Y. (2003). Prolactin-releasing peptide stimulates catecholamine release but not proliferation in rat pheochromocytoma PC12 cells. Neurosci Lett 350, 33-36.

Nanmoku T., Takekoshi K., Fukuda T., Ishii K., Isobe K. \& Kawakami Y. (2005). Stimulation of catecholamine biosynthesis via the PKC pathway by prolactin-releasing peptide in PC12 rat pheochromocytoma cells. $J$ Endocrinol 186, 233-239.

Nässel D.R. \& Wegener C. (2011). A comparative review of short and long neuropeptide F signaling in invertebrates: Any similarities to vertebrate neuropeptide Y signaling?. Peptides 32, 1335-1355.

Navarro V.M., Fernandez-Fernandez R., Castellano J.M., Roa J., Mayen A., Barreiro M.L., et al. (2004). Advanced vaginal opening and precocious activation of the reproductive axis by KiSS-1 peptide, the endogenous ligand of GPR54. J Physiol 561, 379-386.

Navarro V.M., Castellano J.M., Fernandez-Fernandez R., Tovar S., Roa J., Mayen A., et al. (2005). Characterization of the potent luteinizing hormone-releasing activity of KiSS-1 peptide, the natural ligand of GPR54. Endocrinology 146, 156-163.

Navarro V.M., Fernandez-Fernandez R., Nogueiras R., Vigo E., Tovar S., Chartrel N., et al. (2006). Novel role of $26 \mathrm{RFa}$, a hypothalamic RF-amide orexigenic peptide, as putative regulator of the gonadotropic axis. $J$ Physiol 573, 237-249.

Navarro V.M., Ruiz-Pino F., Sanchez-Garrido M.A., Garcia-Galiano D., Hobbs S.J., Manfredi-Lozano M., et al. (2012). Role of neurokinin B in the control of female puberty and its modulation by metabolic status. $J$ Neurosci 32, 2388-2397.

Navarro V.M. \& Tena-Sempere M. (2012). Neuroendocrine control by kisspeptins: role in metabolic regulation of fertility. Nat Rev Endocrinol 8, 40-53.

Navarro V.M. (2013). Interactions between kisspeptins and neurokinin B. Adv Exp Med Biol 784, 325-347.

Neveu C., Lefranc B., Tasseau O., Do-Rego J.C., Bourmaud A., Chan P., et al. (2012). Rational Design of a Low Molecular Weight, Stable, Potent, and Long-Lasting GPR103 Aza-beta(3)-pseudopeptide Agonist. Journal of Medicinal Chemistry 55, 7516-7524.

Newmyer B.A. \& Cline M.A. (2011). Neuropeptide AF is associated with short-term reduced food intake in rats. Behav Brain Res 219, 351-353.

Nguyen P.H., Hammoud H., Halliez S., Pang Y.H., Evrard J., Schmitt M., et al. (2014). Structure-Activity Relationship Study around Guanabenz Identifies Two Derivatives Retaining Antiprion Activity but Having Lost alpha 2-Adrenergic Receptor Agonistic Activity. Acs Chemical Neuroscience 5, 1075-1082. 
Nicklous D.M. \& Simansky K.J. (2003). Neuropeptide FF exerts pro- and anti-opioid actions in the parabrachial nucleus to modulate food intake. Am J Physiol Regul Integr Comp Physiol 285, R1046-1054.

Nieminen M.L., Brandt A., Pietila P. \& Panula P. (2000). Expression of mammalian RF-amide peptides neuropeptide FF (NPFF), prolactin-releasing peptide (PrRP) and the PrRP receptor in the peripheral tissues of the rat. Peptides 21, 1695-1701.

Niida A., Wang Z.X., Tomita K., Oishi S., Tamamura H., Otaka A., et al. (2006). Design and synthesis of downsized metastin (45-54) analogs with maintenance of high GPR54 agonistic activity. Bioorganic \& Medicinal Chemistry Letters 16, 134-137.

Nolasco N., Juarez C., Morgado E., Meza E. \& Caba M. (2012). A circadian clock in the olfactory bulb anticipates feeding during food anticipatory activity. PLoS One 7, e47779.

Nordqvist A., Kristensson L., Johansson K.E., Isaksson da Silva K., Fex T., Tyrchan C., et al. (2014). New Hits as Antagonists of GPR103 Identified by HTS. ACS Medicinal Chemistry Letters 5, 527-532.

Nystedt J.M., Brandt A.M., Mandelin J., Vilim F.S., Ziff E.B. \& Panula P. (2002). Analysis of human neuropeptide FF gene expression. J Neurochem 82, 1330-1342.

Nystedt J.M., Lemberg K., Lintunen M., Mustonen K., Holma R., Kontinen V.K., et al. (2004). Pain- and morphine-associated transcriptional regulation of neuropeptide FF and the G-protein-coupled NPFF2 receptor gene. Neurobiol Dis 16, 254-262.

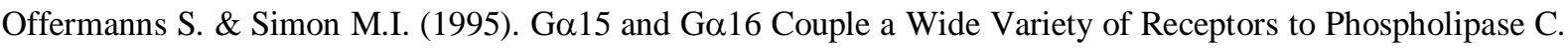
J Biol Chem 270, 15175-15180.

Ohkura S., Uenoyama Y., Yamada S., Homma T., Takase K., Inoue N., et al. (2009). Physiological role of metastin/kisspeptin in regulating gonadotropin-releasing hormone $(\mathrm{GnRH})$ secretion in female rats. Peptides $30,49-56$.

Ohtaki T., Shintani Y., Honda S., Matsumoto H., Hori A., Kanehashi K., et al. (2001). Metastasis suppressor gene KiSS-1 encodes peptide ligand of a G-protein-coupled receptor. Nature 411, 613-617.

Oishi S., Misu R., Tomita K., Setsuda S., Masuda R., Ohno H., et al. (2011). Activation of Neuropeptide FF Receptors by Kisspeptin Receptor Ligands. ACS Med Chem Lett 2, 53-57.

Oishi H., Klausen C., Bentley G.E., Osugi T., Tsutsui K., Gilks C.B., et al. (2012). The Human GonadotropinInhibitory Hormone Ortholog RF-amide Related Peptide-3 Suppresses Gonadotropin-Induced Progesterone Production in Human Granulosa Cells. Endocrinology 153, 3435-3445.

Onaka T. (2004). Neural pathways controlling central and peripheral oxytocin release during stress. $J$ Neuroendocrinol 16, 308-312.

Onaka T., Takayanagi Y. \& Leng G. (2010). Metabolic and stress-related roles of prolactin-releasing peptide. Trends Endocrinol Metab 21, 287-293.

Orsini M.J., Klein M.A., Beavers M.P., Connolly P.J., Middleton S.A. \& Mayo K.H. (2007). Metastin (KiSS-1) mimetics identified from peptide structure-activity relationship-derived pharmacophores and directed small molecule database screening. Journal of Medicinal Chemistry 50, 462-471.

Pampillo M., Camuso N., Taylor J.E., Szereszewski J.M., Ahow M.R., Zajac M., et al. (2009). Regulation of GPR54 signaling by GRK2 and \{beta $\}$-arrestin. Mol Endocrinol 23, 2060-2074.

Panula P., Aarnisalo A.A. \& Wasowicz K. (1996). Neuropeptide FF, a mammalian neuropeptide with multiple functions. Prog Neurobiol 48, 461-487.

Parhar I., Ogawa S. \& Kitahashi T. (2012). RF-amide peptides as mediators in environmental control of GnRH neurons. Prog Neurobiol 98, 176-196.

Parker J.A. \& Bloom S.R. (2012). Hypothalamic neuropeptides and the regulation of appetite. Neuropharmacology 63, 18-30.

Parker R.M., Copeland N.G., Eyre H.J., Liu M., Gilbert D.J., Crawford J., et al. (2000). Molecular cloning and characterisation of GPR74 a novel G-protein coupled receptor closest related to the Y-receptor family. Brain Res Mol Brain Res 77, 199-208.

Pasquier J., Lafont A-G., Tostivint H., Vaudry H., Rousseau K. \& Dufour S. (2012). Comparative evolutionary histories of kisspeptins and kisspeptin receptors in vertebrates reveal both parallel and divergent features. Front Endocrinol (Lausanne) 3, 173.

Patel S.R., Murphy K.G., Thompson E.L., Patterson M., Curtis A.E., Ghatei M.A., et al. (2008). Pyroglutamylated RF-amide peptide 43 stimulates the hypothalamic-pituitary-gonadal axis via gonadotropinreleasing hormone in rats. Endocrinology 149, 4747-4754.

Patterson M., Murphy K.G., Thompson E.L., Patel S., Ghatei M.A. \& Bloom S.R. (2006). Administration of kisspeptin-54 into discrete regions of the hypothalamus potently increases plasma luteinising hormone and testosterone in male adult rats. J Neuroendocrinol 18, 349-354.

Paul M.J., Pyter L.M., Freeman D.A., Galang J. \& Prendergast B.J. (2009). Photic and nonphotic seasonal cues differentially engage hypothalamic kisspeptin and RF-amide-related peptide mRNA expression in Siberian hamsters. J Neuroendocrinol 21, 1007-1014. 
Payza K., Akar C.A. \& Yang H.Y. (1993). Neuropeptide FF receptors: structure-activity relationship and effect of morphine. J Pharmacol Exp Ther 267, 88-94.

Peng J., Tang M., Zhang B.P., Zhang P., Zhong T., Zong T., et al. (2013). Kisspeptin stimulates progesterone secretion via the Erk1/2 mitogen-activated protein kinase signaling pathway in rat luteal cells. Fertil Steril 99, 1436-1443 e1431.

Perry S.J., Yi-Kung Huang E., Cronk D., Bagust J., Sharma R., Walker R.J., et al. (1997). A human gene encoding morphine modulating peptides related to NPFF and FMRF-amide. FEBS Lett 409, 426-430.

Pertovaara A., Ostergard M., Anko M.L., Lehti-Koivunen S., Brandt A., Hong W., et al. (2005). RF-amiderelated peptides signal through the neuropeptide FF receptor and regulate pain-related responses in the rat. Neuroscience 134, 1023-1032.

Peymen K., Watteyne J., Frooninckx L., Schoofs L. \& Beets I. (2014). The FMRF-amide-Like Peptide Family in Nematodes. Front Endocrinol (Lausanne) 5, 90.

Philip F., Kadamur G., Gonzalez Silos R., Woodson J. \& Ross E.M. (2010). Synergistic Activation of Phospholipase C- $\beta 3$ by G $\alpha q$ and G $\beta \gamma$ Describes a Simple Two-State Coincidence Detector. Curr Biol 20, 1327-1335.

Pielecka-Fortuna J., Chu Z. \& Moenter S.M. (2008). Kisspeptin Acts Directly and Indirectly to Increase Gonadotropin-Releasing Hormone Neuron Activity and Its Effects Are Modulated by Estradiol. Endocrinology 149, 1979-1986.

Pierry C., Couve-Bonnaire S., Guilhaudis L., Neveu C., Marotte A., Lefranc B., et al. (2013). Fluorinated Pseudopeptide Analogues of the Neuropeptide 26RFa: Synthesis, Biological, and Structural Studies. Chembiochem 14, 1620-1633.

Pineda R., Garcia-Galiano D., Sanchez-Garrido M.A., Romero M., Ruiz-Pino F., Aguilar E., et al. (2010a). Characterization of the inhibitory roles of RFRP3, the mammalian ortholog of $\mathrm{GnIH}$, in the control of gonadotropin secretion in the rat: in vivo and in vitro studies. Am J Physiol Endocrinol Metab 299, E39-46.

Pineda R., Garcia-Galiano D., Sanchez-Garrido M.A., Romero M., Ruiz-Pino F., Aguilar E. et al. (2010b). Characterization of the Potent Gonadotropin-Releasing Activity of RF9, a Selective Antagonist of RFAmide-Related Peptides and Neuropeptide FF Receptors: Physiological and Pharmacological Implications. Endocrinology 151, 1902-1913.

Pinilla L., Aguilar E., Dieguez C., Millar R.P. \& Tena-Sempere M. (2012). Kisspeptins and reproduction: physiological roles and regulatory mechanisms. Physiol Rev 92, 1235-1316.

Plant T.M., Ramaswamy S. \& Dipietro M.J. (2006). Repetitive activation of hypothalamic G protein-coupled receptor 54 with intravenous pulses of kisspeptin in the juvenile monkey (Macaca mulatta) elicits a sustained train of gonadotropin-releasing hormone discharges. Endocrinology 147, 1007-1013.

Plant T.M. (2015). Neuroendocrine control of the onset of puberty. Front Neuroendocrinol.

Poling M.C., Kim J., Dhamija S. \& Kauffman A.S. (2012). Development, sex steroid regulation, and phenotypic characterization of RF-amide-related peptide (Rfrp) gene expression and RF-amide receptors in the mouse hypothalamus. Endocrinology 153, 1827-1840.

Pompolo S., Pereira A., Estrada K.M. \& Clarke I.J. (2006). Colocalization of kisspeptin and gonadotropinreleasing hormone in the ovine brain. Endocrinology 147, 804-810.

Prevost G., Jeandel L., Arabo A., Coeffier M., El Ouahli M., Picot M., et al. (2015). The hypothalamic neuropeptide 26RFa acts as an incretin to regulate glucose homeostasis. Diabetes.

Price D.A. \& Greenberg M.J. (1977). Structure of a molluscan cardioexcitatory neuropeptide. Science 197, 670671.

Priedkalns J. \& Leiser R. (1998). Female reproductive system. in Dellmann, H. \& Eurell, J. ed (Eds.),Textbook of veterinary histology(pp. 247-269). Baltimore: Williams and Wilkins.

Primeaux S.D., Blackmon C., Barnes M.J., Braymer H.D. \& Bray G.A. (2008). Central administration of the RFamide peptides, QRFP-26 and QRFP-43, increases high fat food intake in rats. Peptides 29, 1994-2000.

Primeaux S.D., Barnes M.J. \& Braymer H.D. (2013). Hypothalamic QRFP: regulation of food intake and fat selection. Horm Metab Res 45, 967-974.

Qi Y., Oldfield B.J. \& Clarke I.J. (2009). Projections of RF-amide-related peptide-3 neurones in the ovine hypothalamus, with special reference to regions regulating energy balance and reproduction. $J$ Neuroendocrinol 21, 690-697.

Quelven I., Roussin A. \& Zajac J.M. (2005). Comparison of pharmacological activities of Neuropeptide FF1 and Neuropeptide FF2 receptor agonists. Eur J Pharmacol 508, 107-114.

Quennell J.H., Rizwan M.Z., Relf H.L. \& Anderson G.M. (2010). Developmental and steroidogenic effects on the gene expression of RF-amide related peptides and their receptor in the rat brain and pituitary gland. $J$ Neuroendocrinol 22, 309-316.

Quennell J.H., Howell C.S., Roa J., Augustine R.A., Grattan D.R. \& Anderson G.M. (2011). Leptin deficiency and diet-induced obesity reduce hypothalamic kisspeptin expression in mice. Endocrinology 152, 1541-1550. 
Rachon D. \& Teede H. (2010). Ovarian function and obesity--interrelationship, impact on women's reproductive lifespan and treatment options. Mol Cell Endocrinol 316, 172-179.

Ramanjaneya M., Karteris E., Chen J., Rucinski M., Ziolkowska A., Ahmed N., et al. (2013). QRFP induces aldosterone production via PKC and T-type calcium channel-mediated pathways in human adrenocortical cells: evidence for a novel role of GPR103. Am J Physiol Endocrinol Metab 305, E1049-1058.

Ramaswamy S., Guerriero K.A., Gibbs R.B. \& Plant T.M. (2008). Structural interactions between kisspeptin and GnRH neurons in the mediobasal hypothalamus of the male rhesus monkey (Macaca mulatta) as revealed by double immunofluorescence and confocal microscopy. Endocrinology 149, 4387-4395.

Ramaswamy S., Seminara S.B., Ali B., Ciofi P., Amin N.A. \& Plant T.M. (2010). Neurokinin B stimulates GnRH release in the male monkey (Macaca mulatta) and is colocalized with kisspeptin in the arcuate nucleus. Endocrinology 151, 4494-4503.

Revel F.G., Saboureau M., Masson-Pevet M., Pevet P., Mikkelsen J.D. \& Simonneaux V. (2006). Kisspeptin mediates the photoperiodic control of reproduction in hamsters. Curr Biol 16, 1730-1735.

Revel F.G., Saboureau M., Pevet P., Simonneaux V. \& Mikkelsen J.D. (2008). RF-amide-related peptide gene is a melatonin-driven photoperiodic gene. Endocrinology 149, 902-912.

Ringel M.D., Hardy E., Bernet V.J., Burch H.B., Schuppert F., Burman K.D., et al. (2002). Metastin receptor is overexpressed in papillary thyroid cancer and activates MAP kinase in thyroid cancer cells. $J$ Clin Endocrinol Metab 87, 2399.

Rizwan M.Z., Porteous R., Herbison A.E. \& Anderson G.M. (2009). Cells expressing RF-amide-related peptide$1 / 3$, the mammalian gonadotropin-inhibitory hormone orthologs, are not hypophysiotropic neuroendocrine neurons in the rat. Endocrinology 150, 1413-1420.

Rizwan M.Z., Poling M.C., Corr M., Cornes P.A., Augustine R.A., Quennell J.H., et al. (2012). RF-amiderelated peptide-3 receptor gene expression in GnRH and kisspeptin neurons and GnRH-dependent mechanism of action. Endocrinology 153, 3770-3779.

Roa J. \& Tena-Sempere M. (2007). KiSS-1 system and reproduction: comparative aspects and roles in the control of female gonadotropic axis in mammals. Gen Comp Endocrinol 153, 132-140.

Roa J., Vigo E., Castellano J.M., Gaytan F., Garcia-Galiano D., Navarro V.M., et al. (2008). Follicle-stimulating hormone responses to kisspeptin in the female rat at the preovulatory period: modulation by estrogen and progesterone receptors. Endocrinology 149, 5783-5790.

Roa J., Garcia-Galiano D., Varela L., Sanchez-Garrido M.A., Pineda R., Castellano J.M., et al. (2009). The mammalian target of rapamycin as novel central regulator of puberty onset via modulation of hypothalamic Kiss1 system. Endocrinology 150, 5016-5026.

Roland B.L., Sutton S.W., Wilson A.J., Luo L., Pyati J., Huvar R., et al. (1999). Anatomical distribution of prolactin-releasing peptide and its receptor suggests additional functions in the central nervous system and periphery. Endocrinology 140, 5736-5745.

Romanovsky A.A. (2007). Thermoregulation: some concepts have changed. Functional architecture of the thermoregulatory system. Am J Physiol Regul Integr Comp Physiol 292, R37-46.

Rometo A.M., Krajewski S.J., Voytko M.L. \& Rance N.E. (2007). Hypertrophy and increased kisspeptin gene expression in the hypothalamic infundibular nucleus of postmenopausal women and ovariectomized monkeys. J Clin Endocrinol Metab 92, 2744-2750.

Rønnekleiv O.K. \& Kelly M.J. (2013). Kisspeptin Excitation of GnRH Neurons. Adv Exp Med Biol 784, 113131.

Rosenkilde M.M. \& Schwartz T.W. (2000). Potency of ligands correlates with affinity measured against agonist and inverse agonists but not against neutral ligand in constitutively active chemokine receptor. Mol Pharmacol 57, 602-609.

Roseweir A.K., Kauffman A.S., Smith J.T., Guerriero K.A., Morgan K., Pielecka-Fortuna J., et al. (2009). Discovery of potent kisspeptin antagonists delineate physiological mechanisms of gonadotropin regulation. $J$ Neurosci 29, 3920-3929.

Roseweir A.K., Katz A.A. \& Millar R.P. (2012). Kisspeptin-10 inhibits cell migration in vitro via a receptorGSK3 beta-FAK feedback loop in HTR8SVneo cells. Placenta 33, 408-415.

Rossi M., Choi S.J., O'Shea D., Miyoshi T., Ghatei M.A. \& Bloom S.R. (1997). Melanin-concentrating hormone acutely stimulates feeding, but chronic administration has no effect on body weight. Endocrinology 138, 351355.

Rothman R.B. (1992). A review of the role of anti-opioid peptides in morphine tolerance and dependence. Synapse 12, 129-138.

Roumy M. \& Zajac J-M. (1996). Effects of neuropeptide FF on intracellular Ca2+ in mouse spinal ganglion neurons. Eur J Pharmacol 306, 291-295.

Roumy M. \& Zajac J.M. (1998). Neuropeptide FF, pain and analgesia. Eur J Pharmacol 345, 1-11.

Roumy M. \& Zajac J. (1999). Neuropeptide FF selectively attenuates the effects of nociceptin on acutely dissociated neurons of the rat dorsal raphe nucleus. Brain Res 845, 208-214. 
Roumy M., Gouarderes C., Mazarguil H. \& Zajac J.M. (2000). Are neuropeptides FF and SF neurotransmitters in the rat? Biochem Biophys Res Commun 275, 821-824.

Roumy M. \& Zajac J-M. (2001). Neuropeptide FF receptors couple to a cholera toxin-sensitive G-protein in rat dorsal raphe neurones. Eur J Pharmacol 417, 45-49.

Saadeldin I.M., Koo O.J., Kang J.T., Kwon D.K., Park S.J., Kim S.J., et al. (2012). Paradoxical effects of kisspeptin: it enhances oocyte in vitro maturation but has an adverse impact on hatched blastocysts during in vitro culture. Reprod Fertil Dev 24, 656-668.

Sahin Z., Canpolat S., Ozcan M., Ozgocer T. \& Kelestimur H. (2015). Kisspeptin antagonist prevents RF9induced reproductive changes in female rats. Reproduction 149, 465-473.

Salehi S., Adeshina I., Chen H., Zirkin B.R., Hussain M.A., Wondisford F., et al. (2015). Developmental and Endocrine Regulation of Kisspeptin Expression in Mouse Leydig Cells. Endocrinology 156, 1514-1522.

Samson W.K. \& Taylor M.M. (2006). Prolactin releasing peptide (PrRP): an endogenous regulator of cell growth. Peptides 27, 1099-1103.

Sanchez-Garrido M.A. \& Tena-Sempere M. (2013). Metabolic control of puberty: roles of leptin and kisspeptins. Horm Behav 64, 187-194.

Sari I.P., Rao A., Smith J.T., Tilbrook A.J. \& Clarke I.J. (2009). Effect of RF-amide-related peptide-3 on luteinizing hormone and follicle-stimulating hormone synthesis and secretion in ovine pituitary gonadotropes. Endocrinology 150, 5549-5556.

Satake H., Hisada M., Kawada T., Minakata H., Ukena K. \& Tsutsui K. (2001). Characterization of a cDNA encoding a novel avian hypothalamic neuropeptide exerting an inhibitory effect on gonadotropin release. Biochem J 354, 379-385.

Satoh N., Ogawa Y., Katsuura G., Hayase M., Tsuji T., Imagawa K., et al. (1997). The arcuate nucleus as a primary site of satiety effect of leptin in rats. Neurosci Lett 224, 149-152.

Schneider J.E. (2004). Energy balance and reproduction. Physiol Behav 81, 289-317.

Schneider J.E., Wise J.D., Benton N.A., Brozek J.M. \& Keen-Rhinehart E. (2013). When do we eat? Ingestive behavior, survival, and reproductive success. Horm Behav 64, 702-728.

Schwartz G.J. \& Zeltser L.M. (2013). Functional organization of neuronal and humoral signals regulating feeding behavior. Annu Rev Nutr 33, 1-21.

Scott G., Ahmad I., Howard K., MacLean D., Oliva C., Warrington S., et al. (2013). Double-blind, randomized, placebo-controlled study of safety, tolerability, pharmacokinetics and pharmacodynamics of TAK-683, an investigational metastin analogue in healthy men. Brit J Clin Pharmacol 75, 381-391.

Seal L.J., Small C.J., Kim M.S., Stanley S.A., Taheri S., Ghatei M.A., et al. (2000). Prolactin releasing peptide (PrRP) stimulates luteinizing hormone (LH) and follicle stimulating hormone (FSH) via a hypothalamic mechanism in male rats. Endocrinology 141, 1909-1912.

Seal L.J., Small C.J., Dhillo W.S., Stanley S.A., Abbott C.R., Ghatei M.A., et al. (2001). PRL-releasing peptide inhibits food intake in male rats via the dorsomedial hypothalamic nucleus and not the paraventricular hypothalamic nucleus. Endocrinology 142, 4236-4243.

Seminara S.B., Messager S., Chatzidaki E.E., Thresher R.R., Acierno J.S., Jr., Shagoury J.K., et al. (2003). The GPR54 gene as a regulator of puberty. $N$ Engl J Med 349, 1614-1627.

Semple R.K., Achermann J.C., Ellery J., Farooqi I.S., Karet F.E., Stanhope R.G., et al. (2005). Two novel missense mutations in $\mathrm{g}$ protein-coupled receptor 54 in a patient with hypogonadotropic hypogonadism. $J$ Clin Endocrinol Metab 90, 1849-1855.

Sexton W.J. \& Jarow J.P. (1997). Effect of diabetes mellitus upon male reproductive function. Urology 49, 508513.

Shahab M., Mastronardi C., Seminara S.B., Crowley W.F., Ojeda S.R. \& Plant T.M. (2005). Increased hypothalamic GPR54 signaling: a potential mechanism for initiation of puberty in primates. Proc Natl Acad Sci U S A 102, 2129-2134.

Silvestre R.A., Egido E.M., Hernandez R. \& Marco J. (2008). Kisspeptin-13 inhibits insulin secretion without affecting glucagon or somatostatin release: study in the perfused rat pancreas. J Endocrinol 196, 283-290.

Simerly R.B. (1998). Organization and regulation of sexually dimorphic neuroendocrine pathways. Behav Brain Res 92, 195-203.

Simerly R.B. (2002). Wired for reproduction: organization and development of sexually dimorphic circuits in the mammalian forebrain. Annu Rev Neurosci 25, 507-536.

Simonin F., Schmitt M., Laulin J.-P., Laboureyras E., Jhamandas J.H., MacTavish D., et al. (2006). RF9, a potent and selective neuropeptide FF receptor antagonist, prevents opioid-induced tolerance associated with hyperalgesia. Proc Natl Acad Sci USA 103, 466-471.

Simonneaux V., Ansel L., Revel F.G., Klosen P., Pevet P. \& Mikkelsen J.D. (2009). Kisspeptin and the seasonal control of reproduction in hamsters. Peptides 30, 146-153.

Simonneaux V., Ancel C., Poirel V.J. \& Gauer F. (2013). Kisspeptins and RFRP-3 Act in Concert to Synchronize Rodent Reproduction with Seasons. Front Neurosci 7, 22. 
Simonnet G. \& Rivat C. (2003). Opioid-induced hyperalgesia: abnormal or normal pain? Neuroreport 14, 1-7.

Singh M. (2014). Mood, food, and obesity. Front Psychol 5, 925.

Singh P., Krishna A., Sridaran R. \& Tsutsui K. (2011a). Immunohistochemical localization of GnRH and RFamide-related peptide-3 in the ovaries of mice during the estrous cycle. J Mol Histol 42, 371-381.

Singh P., Krishna A. \& Tsutsui K. (2011b). Effects of gonadotropin-inhibitory hormone on folliculogenesis and steroidogenesis of cyclic mice. Fertil Steril 95, 1397-1404.

Smith B.K., York D.A. \& Bray G.A. (1994). Chronic cerebroventricular galanin does not induce sustained hyperphagia or obesity. Peptides 15, 1267-1272.

Smith J.T., Cunningham M.J., Rissman E.F., Clifton D.K. \& Steiner R.A. (2005a). Regulation of Kiss1 gene expression in the brain of the female mouse. Endocrinology 146, 3686-3692.

Smith J.T., Dungan H.M., Stoll E.A., Gottsch M.L., Braun R.E., Eacker S.M., et al. (2005b). Differential regulation of KiSS-1 mRNA expression by sex steroids in the brain of the male mouse. Endocrinology 146, 2976-2984.

Smith J.T., Acohido B.V., Clifton D.K. \& Steiner R.A. (2006a). KiSS-1 neurones are direct targets for leptin in the ob/ob mouse. J Neuroendocrinol 18, 298-303.

Smith J.T., Popa S.M., Clifton D.K., Hoffman G.E. \& Steiner R.A. (2006b). Kiss1 neurons in the forebrain as central processors for generating the preovulatory luteinizing hormone surge. $J$ Neurosci 26, 6687-6694.

Smith J.T., Clay C.M., Caraty A. \& Clarke I.J. (2007). KiSS-1 messenger ribonucleic acid expression in the hypothalamus of the ewe is regulated by sex steroids and season. Endocrinology 148, 1150-1157.

Smith J.T., Coolen L.M., Kriegsfeld L.J., Sari I.P., Jaafarzadehshirazi M.R., Maltby M., et al. (2008a). Variation in kisspeptin and RF-amide-related peptide (RFRP) expression and terminal connections to gonadotropinreleasing hormone neurons in the brain: a novel medium for seasonal breeding in the sheep. Endocrinology 149, 5770-5782.

Smith J.T., Rao A., Pereira A., Caraty A., Millar R.P. \& Clarke I.J. (2008b). Kisspeptin is present in ovine hypophysial portal blood but does not increase during the preovulatory luteinizing hormone surge: evidence that gonadotropes are not direct targets of kisspeptin in vivo. Endocrinology 149, 1951-1959.

Smith J.T., Li Q., Pereira A. \& Clarke I.J. (2009a). Kisspeptin neurons in the ovine arcuate nucleus and preoptic area are involved in the preovulatory luteinizing hormone surge. Endocrinology 150, 5530-5538.

Smith J.T., Saleh S.N. \& Clarke I.J. (2009b). Seasonal and cyclical change in the luteinizing hormone response to kisspeptin in the ewe. Neuroendocrinology 90, 283-291.

Smith J.T. \& Clarke I.J. (2010). Gonadotropin inhibitory hormone function in mammals. Trends Endocrinol Metab 21, 255-260.

Smith J.T., Shahab M., Pereira A., Pau K.Y. \& Clarke I.J. (2010). Hypothalamic expression of KISS1 and gonadotropin inhibitory hormone genes during the menstrual cycle of a non-human primate. Biol Reprod 83, 568-577.

Smith J.T., Li Q., Yap K.S., Shahab M., Roseweir A.K., Millar R.P., et al. (2011). Kisspeptin is essential for the full preovulatory $\mathrm{LH}$ surge and stimulates $\mathrm{GnRH}$ release from the isolated ovine median eminence. Endocrinology 152, 1001-1012.

Smith J.T., Young I.R., Veldhuis J.D. \& Clarke I.J. (2012). Gonadotropin-inhibitory hormone (GnIH) secretion into the ovine hypophyseal portal system. Endocrinology 153, 3368-3375.

Smith J.T. (2013). Sex steroid regulation of kisspeptin circuits. Adv Exp Med Biol 784, 275-295.

Son Y.L., Ubuka T., Millar R.P., Kanasaki H. \& Tsutsui K. (2012). Gonadotropin-inhibitory hormone inhibits GnRH-induced gonadotropin subunit gene transcriptions by inhibiting AC/cAMP/PKA-dependent ERK pathway in LbetaT2 cells. Endocrinology 153, 2332-2343.

Song W.J., Mondal P., Wolfe A., Alonso L.C., Stamateris R., Ong B.W., et al. (2014). Glucagon regulates hepatic kisspeptin to impair insulin secretion. Cell Metab 19, 667-681.

Southey B.R., Rodriguez-Zas S.L. \& Sweedler J.V. (2006). Prediction of neuropeptide prohormone cleavages with application to RF-amides. Peptides 27, 1087-1098.

Spampinato S., Trabucco A., Biasiotta A., Biagioni F., Cruccu G., Copani A., et al. (2011). Hyperalgesic activity of kisspeptin in mice. Mol Pain 7, 90.

Spindler-Vomachka M. \& Johnson D.C. (1985). Altered hypothalamic-pituitary function in the adult female rat with streptozotocin-induced diabetes. Diabetologia 28, 38-44.

Stafford L.J., Xia C., Ma W., Cai Y. \& Liu M. (2002). Identification and characterization of mouse metastasissuppressor KiSS1 and its G-protein-coupled receptor. Cancer Res 62, 5399-5404.

Stathatos N., Bourdeau I., Espinosa A.V., Saji M., Vasko V.V., Burman K.D., et al. (2005). KiSS-1/G proteincoupled receptor 54 metastasis suppressor pathway increases myocyte-enriched calcineurin interacting protein 1 expression and chronically inhibits calcineurin activity. J Clin Endocrinol Metab 90, 5432-5440.

Stengel A., Wang L., Goebel-Stengel M. \& Tache Y. (2011). Centrally injected kisspeptin reduces food intake by increasing meal intervals in mice. Neuroreport 22, 253-257. 
Stinus L., Allard M., Gold L. \& Simonnet G. (1995). Changes in CNS neuropeptide FF-like material, pain sensitivity, and opiate dependence following chronic morphine treatment. Peptides 16, 1235-1241.

Sun B., Fujiwara K., Adachi S. \& Inoue K. (2005). Physiological roles of prolactin-releasing peptide. Regul Pept $126,27-33$.

Sun Y.L., Zhang X.Y., He N., Sun T., Zhuang Y., Fang Q., et al. (2012). Neuropeptide FF activates ERK and NF kappa B signal pathways in differentiated SH-SY5Y cells. Peptides 38, 110-117.

Sundblom D.M., Panula P. \& Fyhrquist F. (1995). Neuropeptide FF-like immunoreactivity in human plasma. Peptides 16, 347-350.

Sundblom D.M., Kalso E., Tigerstedt I., Wahlbeck K., Panula P. \& Fyhrquist F. (1997). Neuropeptide FF-like immunoreactivity in human cerebrospinal fluid of chronic pain patients and healthy controls. Peptides 18, 923-927.

Sunter D., Hewson A.K., Lynam S. \& Dickson S.L. (2001). Intracerebroventricular injection of neuropeptide FF, an opioid modulating neuropeptide, acutely reduces food intake and stimulates water intake in the rat. Neurosci Lett 313, 145-148.

Szereszewski J.M., Pampillo M., Ahow M.R., Offermanns S., Bhattacharya M. \& Babwah A.V. (2010). GPR54 regulates ERK1/2 activity and hypothalamic gene expression in a Galpha(q/11) and beta-arrestin-dependent manner. PLoS One 5, e12964.

Tachibana T., Sato M., Takahashi H., Ukena K., Tsutsui K. \& Furuse M. (2005). Gonadotropin-inhibiting hormone stimulates feeding behavior in chicks. Brain Res 1050, 94-100.

Tachibana T. \& Sakamoto T. (2014). Functions of two distinct "prolactin-releasing peptides" evolved from a common ancestral gene. Front Endocrinol (Lausanne) 5, 170.

Takahashi A., Suzuki M., Nakamura Y. \& Sakurai Y. (2010). Preparation of aminotetrahydropyridopyrimidinone derivatives for use in the treatment of stress-related conditions, (Otsuka Pharmaceutical Co., L. ed).

Takahashi K., Yoshinoya A., Arihara Z., Murakami O., Totsune K., Sone M., et al. (2000). Regional distribution of immunoreactive prolactin-releasing peptide in the human brain. Peptides 21, 1551-1555.

Takahashi K., Totsune K., Murakami O., Sone M., Noshiro T., Hayashi Y., et al. (2002). Expression of prolactin-releasing peptide and its receptor in the human adrenal glands and tumor tissues of adrenocortical tumors, pheochromocytomas and neuroblastomas. Peptides 23, 1135-1140.

Takase K., Uenoyama Y., Inoue N., Matsui H., Yamada S., Shimizu M., et al. (2009). Possible role of oestrogen in pubertal increase of Kiss1/kisspeptin expression in discrete hypothalamic areas of female rats. $J$ Neuroendocrinol 21, 527-537.

Takayanagi Y., Matsumoto H., Nakata M., Mera T., Fukusumi S., Hinuma S., et al. (2008). Endogenous prolactin-releasing peptide regulates food intake in rodents. J Clin Invest 118, 4014-4024.

Takayanagi Y. \& Onaka T. (2010). Roles of prolactin-releasing peptide and RF-amide related peptides in the control of stress and food intake. FEBS J 277, 4998-5005.

Takayasu S., Sakurai T., Iwasaki S., Teranishi H., Yamanaka A., Williams S.C., et al. (2006). A neuropeptide ligand of the G protein-coupled receptor GPR103 regulates feeding, behavioral arousal, and blood pressure in mice. Proc Natl Acad Sci U S A 103, 7438-7443.

Talmont F., Garcia L.P., Mazarguil H., Zajac J.M. \& Mollereau C. (2009). Characterization of two novel tritiated radioligands for labelling Neuropeptide FF (NPFF(1) and NPFF(2)) receptors. Neurochem Int 55, 815-819.

Talmont F., Mouledous L., Piedra-Garcia L., Schmitt M., Bihel F., Bourguignon J.J., et al. (2010). Pharmacological characterization of the mouse NPFF2 receptor. Peptides 31, 215-220.

Tang-Christensen M., Holst J.J., Hartmann B. \& Vrang N. (1999). The arcuate nucleus is pivotal in mediating the anorectic effects of centrally administered leptin. Neuroreport 10, 1183-1187.

Teles M.G., Bianco S.D., Brito V.N., Trarbach E.B., Kuohung W., Xu S., et al. (2008). A GPR54-activating mutation in a patient with central precocious puberty. $N$ Engl J Med 358, 709-715.

Terao Y., Kumano S., Takatsu Y., Hattori M., Nishimura A., Ohtaki T., et al. (2004). Expression of KiSS-1, a metastasis suppressor gene, in trophoblast giant cells of the rat placenta. Biochim Biophys Acta 1678, 102110.

Thompson E.L., Patterson M., Murphy K.G., Smith K.L., Dhillo W.S., Todd J.F., et al. (2004). Central and peripheral administration of kisspeptin-10 stimulates the hypothalamic-pituitary-gonadal axis. $J$ Neuroendocrinol 16, 850-858.

Thompson E.L., Murphy K.G., Patterson M., Bewick G.A., Stamp G.W., Curtis A.E., et al. (2006). Chronic subcutaneous administration of kisspeptin-54 causes testicular degeneration in adult male rats. Am J Physiol Endocrinol Metab 291, E1074-1082.

Thuau R., Guilhaudis L., Segalas-Milazzo I., Chartrel N., Oulyadi H., Boivin S., et al. (2005). Structural studies on 26RFa, a novel human RF-amide-related peptide with orexigenic activity. Peptides 26, 779-789.

Tolson K.P., Garcia C., Yen S., Simonds S., Stefanidis A., Lawrence A., et al. (2014). Impaired kisspeptin signaling decreases metabolism and promotes glucose intolerance and obesity. J Clin Invest 124, 3075-3079. 
Tomita K., Niida A., Oishi S., Ohno H., Cluzeau J., Navenot J.-M., et al. (2006). Structure-activity relationship study on small peptidic GPR54 agonists. Bioorganic \& Medicinal Chemistry 14, 7595-7603.

Tomita K., Oishi S., Cluzeau J., Ohno H., Navenot J.-M., Wang Z.-X., et al. (2007). SAR and QSAR studies on the n-terminally acylated pentapeptide agonists for GPR54. Journal of Medicinal Chemistry 50, 3222-3228.

Tomita K., Oishi S., Ohno H. \& Fujii N. (2008a). Structure-activity relationship study and NMR analysis of fluorobenzoyl pentapeptide GPR54 agonists. Biopolymers 90, 503-511.

Tomita K., Oishi S., Ohno H., Peiper S.C. \& Fujii N. (2008b). Development of Novel G-Protein-Coupled Receptor 54 Agonists with Resistance to Degradation by Matrix Metalloproteinase. Journal of Medicinal Chemistry 51, 7645-7649.

Tovar S., Vazquez M.J., Navarro V.M., Fernandez-Fernandez R., Castellano J.M., Vigo E., et al. (2006). Effects of single or repeated intravenous administration of kisspeptin upon dynamic LH secretion in conscious male rats. Endocrinology 147, 2696-2704.

True C., Kirigiti M.A., Kievit P., Grove K.L. \& Smith M.S. (2011). Leptin is not the critical signal for kisspeptin or luteinising hormone restoration during exit from negative energy balance. J Neuroendocrinol 23, 10991112.

Tsutsui K., Saigoh E., Ukena K., Teranishi H., Fujisawa Y., Kikuchi M., et al. (2000). A novel avian hypothalamic peptide inhibiting gonadotropin release. Biochem Biophys Res Commun 275, 661-667.

Tsutsui K., Saigoh E., Yin H., Ubuka T., Chowdhury V.S., Osugi T., et al. (2009). A new key neurohormone controlling reproduction, gonadotrophin-inhibitory hormone in birds: discovery, progress and prospects. $J$ Neuroendocrinol 21, 271-275.

Tsutsui K., Bentley G.E., Kriegsfeld L.J., Osugi T., Seong J.Y. \& Vaudry H. (2010). Discovery and evolutionary history of gonadotrophin-inhibitory hormone and kisspeptin: new key neuropeptides controlling reproduction. J Neuroendocrinol 22, 716-727.

Tsutsui K., Ubuka T., Bentley G.E. \& Kriegsfeld L.J. (2012). Gonadotropin-inhibitory hormone (GnIH): discovery, progress and prospect. Gen Comp Endocrinol 177, 305-314.

Ubuka T., Bentley G.E., Ukena K., Wingfield J.C. \& Tsutsui K. (2005). Melatonin induces the expression of gonadotropin-inhibitory hormone in the avian brain. Proc Natl Acad Sci U S A 102, 3052-3057.

Ubuka T., Lai H., Kitani M., Suzuuchi A., Pham V., Cadigan P.A., et al. (2009a). Gonadotropin-inhibitory hormone identification, cDNA cloning, and distribution in rhesus macaque brain. J Comp Neurol 517, 841855.

Ubuka T., Morgan K., Pawson A.J., Osugi T., Chowdhury V.S., Minakata H., et al. (2009b). Identification of human GnIH homologs, RFRP-1 and RFRP-3, and the cognate receptor, GPR147 in the human hypothalamic pituitary axis. PLoS One 4, e8400.

Ubuka T., Inoue K., Fukuda Y., Mizuno T., Ukena K., Kriegsfeld L.J., et al. (2012a). Identification, expression, and physiological functions of Siberian hamster gonadotropin-inhibitory hormone. Endocrinology 153, 373385.

Ubuka T., Son Y.L., Tobari Y. \& Tsutsui K. (2012b). Gonadotropin-inhibitory hormone action in the brain and pituitary. Front Endocrinol (Lausanne) 3, 148.

Ubuka T., Son Y.L., Tobari Y., Narihiro M., Bentley G.E., Kriegsfeld L.J., et al. (2014). Central and direct regulation of testicular activity by gonadotropin-inhibitory hormone and its receptor. Front Endocrinol (Lausanne) 5, 8.

Ukena K. \& Tsutsui K. (2001). Distribution of novel RF-amide-related peptide-like immunoreactivity in the mouse central nervous system. Neurosci Lett 300, 153-156.

Ukena K., Iwakoshi E., Minakata H. \& Tsutsui K. (2002). A novel rat hypothalamic RF-amide-related peptide identified by immunoaffinity chromatography and mass spectrometry. FEBS Lett 512, 255-258.

Ukena K. \& Tsutsui K. (2005). A new member of the hypothalamic RF-amide peptide family, LPXRF-amide peptides: Structure, localization and function. Mass Spectrometry Reviews 24, 469- 486.

Ukena K., Tachibana T., Tobari Y., Leprince J., Vaudry H. \& Tsutsui K. (2013). Identification, localization and function of a novel neuropeptide, 26RFa, and its cognate receptor, GPR103, in the avian hypothalamus. Gen Comp Endocrinol 190, 42-46.

van Harmelen V., Dicker A., Sjolin E., Blomqvist L., Wiren M., Hoffstedt J., et al. (2010). Effects of pain controlling neuropeptides on human fat cell lipolysis. Int J Obes (Lond) 34, 1333-1340.

Varghese B.V., Koohestani F., McWilliams M., Colvin A., Gunewardena S., Kinsey W.H., et al. (2013). Loss of the repressor REST in uterine fibroids promotes aberrant $\mathrm{G}$ protein-coupled receptor 10 expression and activates mammalian target of rapamycin pathway. Proc Natl Acad Sci U S A 110, 2187-2192.

Vikman J. \& Ahren B. (2009). Inhibitory effect of kisspeptins on insulin secretion from isolated mouse islets. Diabetes Obes Metab 11 Suppl 4, 197-201.

Vilim F.S., Aarnisalo A.A., Nieminen M.L., Lintunen M., Karlstedt K., Kontinen V.K., et al. (1999). Gene for pain modulatory neuropeptide NPFF: induction in spinal cord by noxious stimuli. Mol Pharmacol 55, 804811. 
Volkow N.D., Wang G.J. \& Baler R.D. (2011). Reward, dopamine and the control of food intake: implications for obesity. Trends Cogn Sci 15, 37-46.

Wahab F., Riaz T. \& Shahab M. (2011). Study on the effect of peripheral kisspeptin administration on basal and glucose-induced insulin secretion under fed and fasting conditions in the adult male rhesus monkey (Macaca mulatta). Horm Metab Res 43, 37-42.

Wahab F., Salahuddin H., Anees M., Leprince J., Vaudry H., Tena-Sempere M., et al. (2012). Study of the effect of 26RF- and 43RF-amides on testosterone and prolactin secretion in the adult male rhesus monkey (Macaca mulatta). Peptides 36, 23-28.

Wahab F., Atika B. \& Shahab M. (2013). Kisspeptin as a link between metabolism and reproduction: evidences from rodent and primate studies. Metabolism 62, 898-910.

Wakabayashi Y., Nakada T., Murata K., Ohkura S., Mogi K., Navarro V.M., et al. (2010). Neurokinin B and dynorphin $\mathrm{A}$ in kisspeptin neurons of the arcuate nucleus participate in generation of periodic oscillation of neural activity driving pulsatile gonadotropin-releasing hormone secretion in the goat. J Neurosci 30, 31243132.

Walker R.J., Papaioannou S. \& Holden-Dye L. (2009). A review of FMRF-amide- and RF-amide-like peptides in metazoa. Invert Neurosci 9, 111-153.

Wang Y., Wang C.Y., Wu Y., Huang G., Li J. \& Leung F.C. (2012). Identification of the receptors for prolactinreleasing peptide (PrRP) and Carassius RF-amide peptide (C-RFa) in chickens. Endocrinology 153, 18611874.

Watanabe T.K., Suzuki M., Yamasaki Y., Okuno S., Hishigaki H., Ono T., et al. (2005). Mutated G-proteincoupled receptor GPR10 is responsible for the hyperphagia/dyslipidaemia/obesity locus of Dmo1 in the OLETF rat. Clin Exp Pharmacol Physiol 32, 355-366.

Watanobe H. (2001). In vivo release of prolactin-releasing peptide in rat hypothalamus in association with luteinizing hormone and prolactin surges. Neuroendocrinology 74, 359-366.

Weber E., Evans C.J., Samuelsson S.J. \& Barchas J.D. (1981). Novel peptide neuronal system in rat brain and pituitary. Science 214, 1248-1251.

Welch S.K., O'Hara B.F., Kilduff T.S. \& Heller H.C. (1995). Sequence and tissue distribution of a candidate Gcoupled receptor cloned from rat hypothalamus. Biochem Biophys Res Commun 209, 606-613.

Wettschureck N. \& Offermanns S. (2005). Mammalian G Proteins and Their Cell Type Specific Functions. Physiol Rev 85, 1159-1204.

Wiegand S.J. \& Terasawa E. (1982). Discrete lesions reveal functional heterogeneity of suprachiasmatic structures in regulation of gonadotropin secretion in the female rat. Neuroendocrinology 34, 395-404.

Williams K.W. \& Elmquist J.K. (2012). From neuroanatomy to behavior: central integration of peripheral signals regulating feeding behavior. Nat Neurosci 15, 1350-1355.

Wisler J.W., Xiao K., Thomsen A.R. \& Lefkowitz R.J. (2014). Recent developments in biased agonism. Curr Opin Cell Biol 27, 18-24.

Wolfe J.T., Wang H., Howard J., Garrison J.C. \& Barrett P.Q. (2003). T-type calcium channel regulation by specific G-protein $\beta \gamma$ subunits. Nature 424, 209-213.

Wright D.E. \& Jennes L. (1993). Origin of noradrenergic projections to GnRH perikarya-containing areas in the medial septum-diagonal band and preoptic area. Brain Res 621, 272-278.

Wu C.H., Tao P.L. \& Huang E.Y. (2010). Distribution of neuropeptide FF (NPFF) receptors in correlation with morphine-induced reward in the rat brain. Peptides 31, 1374-1382.

Xu J., Kirigiti M.A., Grove K.L. \& Smith M.S. (2009). Regulation of food intake and gonadotropin-releasing hormone/luteinizing hormone during lactation: role of insulin and leptin. Endocrinology 150, 4231-4240.

Xu Z., Kaga S., Mochiduki A., Tsubomizu J., Adachi S., Sakai T., et al. (2012). Immunocytochemical localization of kisspeptin neurons in the rat forebrain with special reference to sexual dimorphism and interaction with GnRH neurons. Endocr J 59, 161-171.

Yamada M., Ozawa A., Ishii S., Shibusawa N., Hashida T., Ishizuka T., et al. (2001). Isolation and characterization of the rat prolactin-releasing peptide gene: multiple TATA boxes in the promoter region. Biochem Biophys Res Commun 281, 53-56.

Yamada S., Uenoyama Y., Kinoshita M., Iwata K., Takase K., Matsui H., et al. (2007). Inhibition of metastin (kisspeptin-54)-GPR54 signaling in the arcuate nucleus-median eminence region during lactation in rats. Endocrinology 148, 2226-2232.

Yamaji T., Dierschke D.J., Hotchkiss J., Bhattacharya A.N., Surve A.H. \& Knobil E. (1971). Estrogen induction of LH release in the rhesus monkey. Endocrinology 89, 1034-1041.

Yamamoto T., Wada T. \& Miyazaki R. (2008). Analgesic effects of intrathecally administered 26RFa, an intrinsic agonist for GPR103, on formalin test and carrageenan test in rats. Neuroscience 157, 214-222.

Yamamoto T., Miyazaki R. \& Yamada T. (2009). Intracerebroventricular administration of 26RFa produces an analgesic effect in the rat formalin test. Peptides 30, 1683-1688. 
Yamamoto T., Miyazaki R., Yamada T. \& Shinozaki T. (2011). Anti-allodynic effects of intrathecally and intracerebroventricularly administered $26 \mathrm{RFa}$, an intrinsic agonist for GRP103, in the rat partial sciatic nerve ligation model. Peptides 32, 1262-1269.

Yamamoto Y., Ueta Y., Serino R., Nomura M., Shibuya I. \& Yamashita H. (2000). Effects of food restriction on the hypothalamic prepro-orexin gene expression in genetically obese mice. Brain Res Bull 51, 515-521.

Yamashita M., Takayanagi Y., Yoshida M., Nishimori K., Kusama M. \& Onaka T. (2013). Involvement of prolactin-releasing peptide in the activation of oxytocin neurones in response to food intake. $J$ Neuroendocrinol 25, 455-465.

Yang H.Y., Fratta W., Majane E.A. \& Costa E. (1985). Isolation, sequencing, synthesis, and pharmacological characterization of two brain neuropeptides that modulate the action of morphine. Proc Natl Acad Sci U S A 82, 7757-7761.

Yang H.Y. \& Iadarola M.J. (2003). Activation of spinal neuropeptide FF and the neuropeptide FF receptor 2 during inflammatory hyperalgesia in rats. Neuroscience 118, 179-187.

Yang H.Y. \& Iadarola M.J. (2006). Modulatory roles of the NPFF system in pain mechanisms at the spinal level. Peptides 27, 943-952.

Yang H.Y., Tao T. \& Iadarola M.J. (2008). Modulatory role of neuropeptide FF system in nociception and opiate analgesia. Neuropeptides 42, 1-18.

Yang H.Y.T. \& Martin B.M. (1995). Isolation and characterization of a neuropeptide FF-like from brain and spinal cord of rat. Society for Neurosciences 21, 760.

Yano T., Iijima N., Kakihara K., Hinuma S., Tanaka M. \& Ibata Y. (2003). Localization and neuronal response of RF-amide related peptides in the rat central nervous system. Brain Res 982, 156-167.

Yeo G.S. \& Heisler L.K. (2012). Unraveling the brain regulation of appetite: lessons from genetics. Nat Neurosci 15, 1343-1349.

Yeo S.H. \& Herbison A.E. (2011). Projections of arcuate nucleus and rostral periventricular kisspeptin neurons in the adult female mouse brain. Endocrinology 152, 2387-2399.

Yim G.K. \& Lowy M.T. (1984). Opioids, feeding, and anorexias. Fed Proc 43, 2893-2897.

Yoshida H., Habata Y., Hosoya M., Kawamata Y., Kitada C. \& Hinuma S. (2003). Molecular properties of endogenous RF-amide-related peptide-3 and its interaction with receptors. Biochim Biophys Acta 1593, 151157.

Yun S., Kim D-K., Furlong M., Hwang J-I., Vaudry H. \& Seong J.Y. (2014). Does kisspeptin belong to the proposed RF-amide peptide family ?. Front Endocrinol (Lausanne) 5, 134.

Zajac J.-M. \& Roques B.P. (1989). Properties required for reversible and irreversible radiolabelled probes for selective characterization of brain receptors and peptidases by autoradiography. in Shariff, N. A. \& Lewis, M. E. ed (Eds.),Brain imaging: Techniques and Application(pp. 18-35). Chichester: Ellis Horwood.

Zajac J.M., Latapie J.P. \& Frances B. (2000). Opposing interplay between Neuropeptide FF and nitric oxide in antinociception and hypothermia. Peptides 21, 1209-1213.

Zajac M., Law J., Cvetkovic D.D., Pampillo M., McColl L., Pape C., et al. (2011). GPR54 (KISS1R) transactivates EGFR to promote breast cancer cell invasiveness. PLoS One 6, e21599.

Zamponi G.W. \& Currie K.P.M. (2013). Regulation of CaV2 calcium channels by G protein coupled receptors. Biochim Biophys Acta 1828, 1629-1643.

Zeng Z., McDonald T.P., Wang R., Liu Q. \& Austin C.P. (2003). Neuropeptide FF receptor 2 (NPFF2) is localized to pain-processing regions in the primate spinal cord and the lower level of the medulla oblongata. $J$ Chem Neuroanat 25, 269-278.

Zhang C., Roepke T.A., Kelly M.J. \& Rønnekleiv, O.K. (2008). Kisspeptin Depolarizes GonadotropinReleasing Hormone Neurons through Activation of TRPC-Like Cationic Channels. J Neurosci 28, 44234434.

Zhang C., Bosch M.A., Ronnekleiv O.K. \& Kelly M.J. (2009). Gamma-aminobutyric acid B receptor mediated inhibition of gonadotropin-releasing hormone neurons is suppressed by kisspeptin-G protein-coupled receptor 54 signaling. Endocrinology 150, 2388-2394.

Zhang C., Bosch M.A., Rønnekleiv O.K. \& Kelly M.J. (2013a). Kisspeptin Activation of TRPC4 Channels in Female GnRH Neurons Requires PIP2 Depletion and cSrc Kinase Activation. Endocrinology 154, 27722783.

Zhang C., Rønnekleiv O.K. \& Kelly M.J. (2013b). Kisspeptin inhibits a slow afterhyperpolarization current via protein kinase $\mathrm{C}$ and reduces spike frequency adaptation in GnRH neurons. Am J Physiol Endocrinol Metab 304, E1237-E1244.

Zhang Q., Qiu P., Arreaza M.G., Simon J.S., Golovko A., Laverty M., et al. (2007). P518/Qrfp sequence polymorphisms in SAMP6 osteopenic mouse. Genomics 90, 629-635.

Zhang X.B. \& Spergel D.J. (2012). Kisspeptin inhibits high-voltage activated Ca2+ channels in GnRH neurons via multiple Ca2+ influx and release pathways. Neuroendocrinol 96, 68-80. 
Zhang Y., Proenca R., Maffei M., Barone M., Leopold L. \& Friedman J.M. (1994). Positional cloning of the mouse obese gene and its human homologue. Nature 372, 425-432.

Zhao L., Zhong M., Xue H.L., Ding J.S., Wang S., Xu J.H., et al. (2014). Effect of RFRP-3 on reproduction is sex- and developmental status-dependent in the striped hamster (Cricetulus barabensis). Gene 547, 273-279.

Zhao S., Zhu E., Yang C., Bentley G.E., Tsutsui K. \& Kriegsfeld L.J. (2010). RF-amide-related peptide and messenger ribonucleic acid expression in mammalian testis: association with the spermatogenic cycle. Endocrinology 151, 617-627.

Zhou D., Zhuo Y., Che L., Lin Y., Fang Z. \& Wu D. (2014). Nutrient restriction induces failure of reproductive function and molecular changes in hypothalamus-pituitary-gonadal axis in postpubertal gilts. Mol Biol Rep $41,4733-4742$.

Zhuo Y., Zhou D., Che L., Fang Z., Lin Y. \& Wu D. (2014). Feeding prepubescent gilts a high-fat diet induces molecular changes in the hypothalamus-pituitary-gonadal axis and predicts early timing of puberty. Nutrition 30, 890-896. 


\section{Legends to Figures}

Figure 1 : Dipeptides, arginine and non-natural amino acids as ligands of NPFF receptors.

See text for further details on structural and pharmacological properties of the compounds.

Figure 2 : Non-peptidic small molecules as ligands of NPFF receptors.

See text for further details on structural and pharmacological properties of the compounds.

\section{Figure 3 : Non-peptidic PrRP-R antagonist.}

The binding affinity constant $\mathrm{K}_{\mathrm{i}}$ for Compound $\mathbf{2 3 8 6}$ was derived from competition studies conducted on membranes from PrRPR-expressing HEK cells using $\left[{ }^{3} \mathrm{H}\right]-\mathrm{PrRP}$ as the tracer (WO/2010/137738A1 patent). The IC $_{50}$ value (the drug concentration that inhibits half of specific radioligand binding) was converted into a $\mathrm{K}_{\mathrm{i}}$ value using the Cheng-Prusoff formula.

\section{Figure 4 : Pentapeptides and peptidomimetics as Kiss1R agonists.}

The agonist activity of the compounds was evaluated through their ability to induce an intracellular calcium mobilization in Kiss1R-transfected $\mathrm{CHO}$ or HEK cells. $\mathrm{EC}_{50}$ values for FM053a (Tomita et al., 2006), FTM080 (or compound 1 in Tomita et al., 2008b), FTM145 (or compound 19 in Tomita et al., 2008b), TAK-683 (or compound 22 in Asami et al., 2014) and Compound 17 (Beltramo et al., 2015) were derived from dose-response curves to define the concentrations of peptides leading to half-maximal effects.

\section{Figure 5 : Non-peptidic Kiss1R antagonists.}

IC $_{50}$ values for compounds 15a (Kobayashi et al., 2010b) and 91 (Kobayashi et al., 2010a) were defined as the concentrations that displaced half specific $\left[{ }^{125} \mathrm{I}\right]-\mathrm{Kp} 14$ binding on membranes of Kiss1R-expressing CHO cells.

\section{Figure 6 : Pseudopeptidic agonists and antagonists of the QRFP receptor.}

EC $_{50}$ values for LV-2172 (Neveu et al., 2012) and LV-2098 (Pierry et al., 2013) were defined as their potencies, as agonists, to promote an increase in intracellular calcium levels in $\mathrm{G} \alpha_{16} /$ Kiss1R transfected $\mathrm{CHO}$ cells. $\mathrm{IC}_{50}$ values for the indole derivative (compound 7 in WO/2010/126164 patent), compound 25e (Georgsson et al., 2014) and the sulfonamide 2 
(Norqvist et al., 2014) were from [ $\left.{ }^{125} \mathrm{I}\right]-\mathrm{QRFP} 43$ binding inhibition experiments performed on membranes of QRFPR-expressing cells.

Figure 7. Anatomical distribution of RF-amide peptides and their cognate receptors in rodent (rat and mouse) brain areas involved in energy homeostasis.

Highlighted brain structures have been selected on the basis of their known or putative roles in feeding behaviour and regulation of energy homeostasis in Mammals and of their enrichment in RF-amide peptides (left panel) and their cognate receptors (right panel), as afforded from distribution studies in mouse brain. When specified in the literature, the localization of RF-amide peptide-containing cell bodies is privileged. Colored areas refer to distinct anatomical structures according to MeSH database : hypothalamic nuclei (light blue); pons/medulla oblongata nuclei (pink); basal ganglia (yellow); tegmentum mesencephali nuclei (green); telencephalon nuclei (orange); cortical areas (light orange). Details on the preferential distribution of QRFP-R1 and QRFP-R2 receptor isoforms are provided in Section 7.6.1. Abbreviations are listed in the corresponding section.

Figure 8 : Anatomical distribution of RF-amide peptides and their cognate receptors in rodent (rat and mouse) areas involved in reproduction.

Highlighted brain structures have been selected on the basis of their known or putative roles in the control of reproduction in Mammals and of their enrichment in RF-amide peptides (left panel) and their cognate receptors (right panel), as afforded from distribution studies in mouse brain. When specified in the literature, the localization of RF-amide peptide-containing cell bodies is privileged. Colored areas refer to distinct anatomical structures according to MeSH database : hypothalamic nuclei (light blue); telencephalon nuclei (orange). Details on the differential distribution of QRFP-R1 and QRFP-R2 receptor isoforms are provided in Section 8.6.1. Abbreviations are listed in the corresponding section.

Figure 9 : Anatomical distribution of RF-amide peptides and their cognate receptors in rodent (rat and mouse) central and peripheral nervous system areas involved in nociception.

Highlighted brain structures have been selected on the basis of their known or putative roles in nociception and pain modulation in Mammals and of their enrichment in RF-amide peptides (left panel) and their cognate receptors (right panel), as afforded from distribution studies in mouse brain. When specified in the literature, localization of RF-amide peptide- 
containing cell bodies is privileged. Colored areas refer to distinct anatomical structures according to $\mathrm{MeSH}$ database : hypothalamic nuclei (light blue); pons/medulla oblongata nuclei (pink); basal ganglia (yellow); tegmentum mesencephali nuclei (green); epithalamus (red); thalamic nuclei (blue); spinal cord and DRG (light grey). To note that the expression of NPFF1R in the dorsal horn of the spinal cord depends on rodent strains (Bonini et al., 2000; Liu et al., 2001; Gouarderes et al., 2004a). The expression of NPFF in the DRG is still a matter of controversy (Ayachi and Simonin 2014, for a review). The distribution of QRFP-R1 and QRFP-R2 isoforms in pain-related areas is detailed in the original papers by Fukusumi et al., 2003; Kampe et al., 2006 and Takayasu et al., 2006. Abbreviations are listed in the corresponding section. 
Table 1. RF-amide precursor proteins, RF-amide neuropeptides and their receptors in four mammalian species.

\begin{tabular}{|c|c|c|c|c|c|}
\hline & Peptide & Species & Sequence & References & $\mathbf{R}$ \\
\hline$\frac{\pi}{2}$ & $\begin{array}{l}\text { NPFF } \\
\text { (NPSF) } \\
\text { NPAF }\end{array}$ & $\begin{array}{l}\text { Human, bovine } \\
\text { Rat, mouse } \\
\text { Human } \\
\text { Rat } \\
\text { Mouse } \\
\text { Human, rat, mouse } \\
\text { Human } \\
\text { Bovine } \\
\text { Rat (P) } \\
\text { Mouse (P) }\end{array}$ & 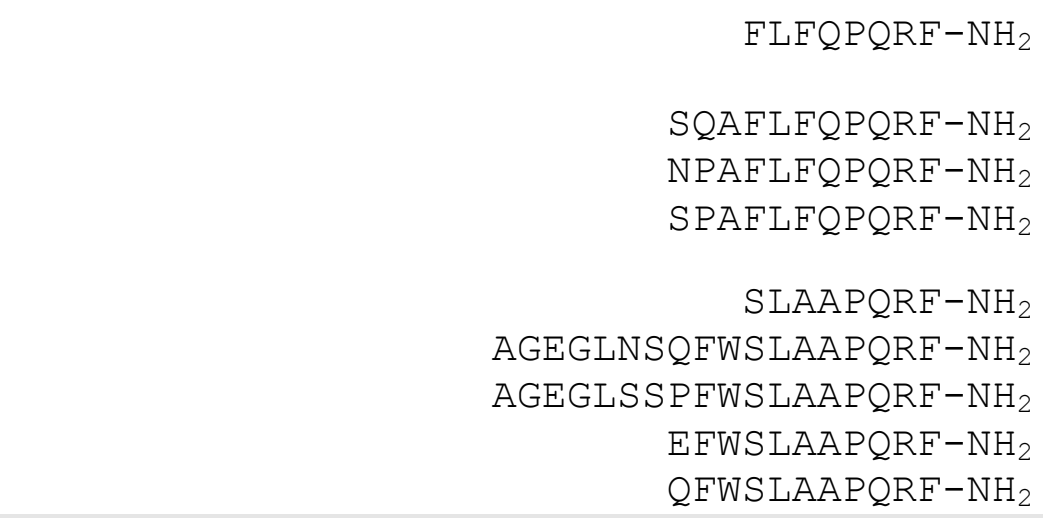 & $\begin{array}{l}\text { Sundblom et al, 1995; Yang et al, } 1985 \\
\text { Bonnard et al, } 2001 \\
\text { Bonnard et al, } 2003 \\
\text { Bonnard et al, } 2001 \\
\text { Bonnard et al, } 2003 \\
\text { Burlet-Schiltz et al, 2002; Bonnard et al, } 2001 \\
\text { Bonnard et al, 2003 } \\
\text { Yang et al, 1985 } \\
\text { Roumy et al, } 2000 \\
\text { Roumy et al, } 2000\end{array}$ & 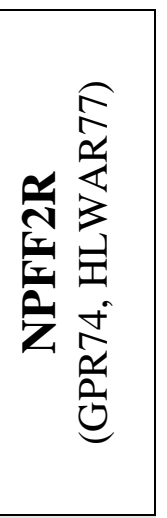 \\
\hline 竞 & $\begin{array}{c}\text { RFRP-1 } \\
\text { (GnIH, ) } \\
\text { NPSF) }\end{array}$ & $\begin{array}{l}\text { Human } \\
\text { Rat, mouse (P) } \\
\text { Human }(\mathrm{P}) \\
\text { Bovine } \\
\text { Rat }(\mathrm{P}) \\
\text { Mouse (P) } \\
\text { Human } \\
\text { Bovine } \\
\text { Rat } \\
\text { Mouse (P) }\end{array}$ & 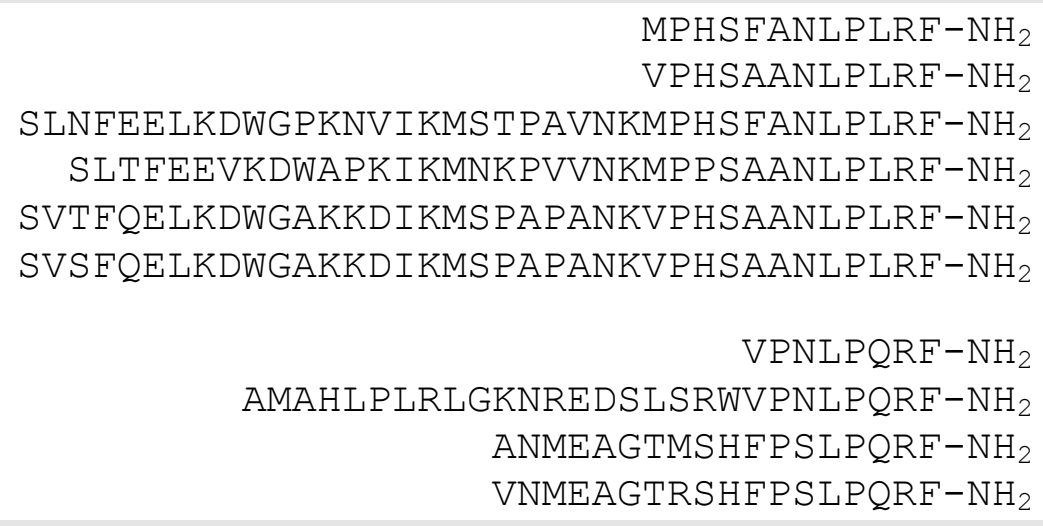 & $\begin{array}{l}\text { Ubuka et al, 2009 } \\
\text { Hinuma et al, } 2000 \\
\text { Fukusumi et al, } 2001 \text {; Liu et al, } 2001 \\
\text { Fukusumi et al, } 2001 \\
\text { Fukusumi et al, 2001; Liu et al, } 2001 \\
\text { Liu et al, 2001; Ukena \& Tsutsui } 2005 \\
\\
\text { Ubuka et al, 2009 } \\
\text { Yoshida et al, } 2003 \\
\text { Ukena et al, 2002 } \\
\text { Ukena \& Tsutsui } 2005\end{array}$ & 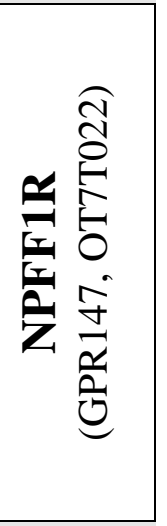 \\
\hline 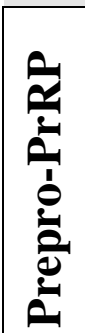 & PrRP-31 & $\begin{array}{l}\text { Human }(\mathrm{P}) \\
\text { Bovine } \\
\text { Rat, mouse }(\mathrm{P}) \\
\text { Human }(\mathrm{P}) \\
\text { Bovine } \\
\text { Rat, mouse }(\mathrm{P})\end{array}$ & $\begin{array}{r}\text { TPDINPAWYASRGIRPVGRF-NH } \\
\text { TPDINPAWYAGRGIRPVGRF- } \mathrm{NH}_{2} \\
\text { TPDINPAWYTGRGIRPVGRF- } \mathrm{NH}_{2} \\
\text { SRTHRHSMEIRTPDINPAWYASRGIRPVGRF }-\mathrm{NH}_{2} \\
\text { SRAHRHSMEIRTPDINPAWYAGRGIRPVGRF- } \mathrm{NH}_{2} \\
\text { SRAHQHSMETRTPDINPAWYTGRGIRPVGRF- } \mathrm{NH}_{2}\end{array}$ & $\begin{array}{l}\text { Hinuma et al, } 1998 \\
\text { Hinuma et al, } 1998 \\
\text { Hinuma et al, 1998; Tachibana et al, } 2014 \\
\text { Hinuma et al, } 1998 \\
\text { Hinuma et al, } 1998 \\
\text { Hinuma et al, 1998; Tachibana et al, } 2014\end{array}$ & 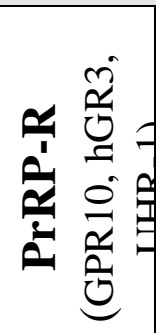 \\
\hline
\end{tabular}


Table 1. Continued

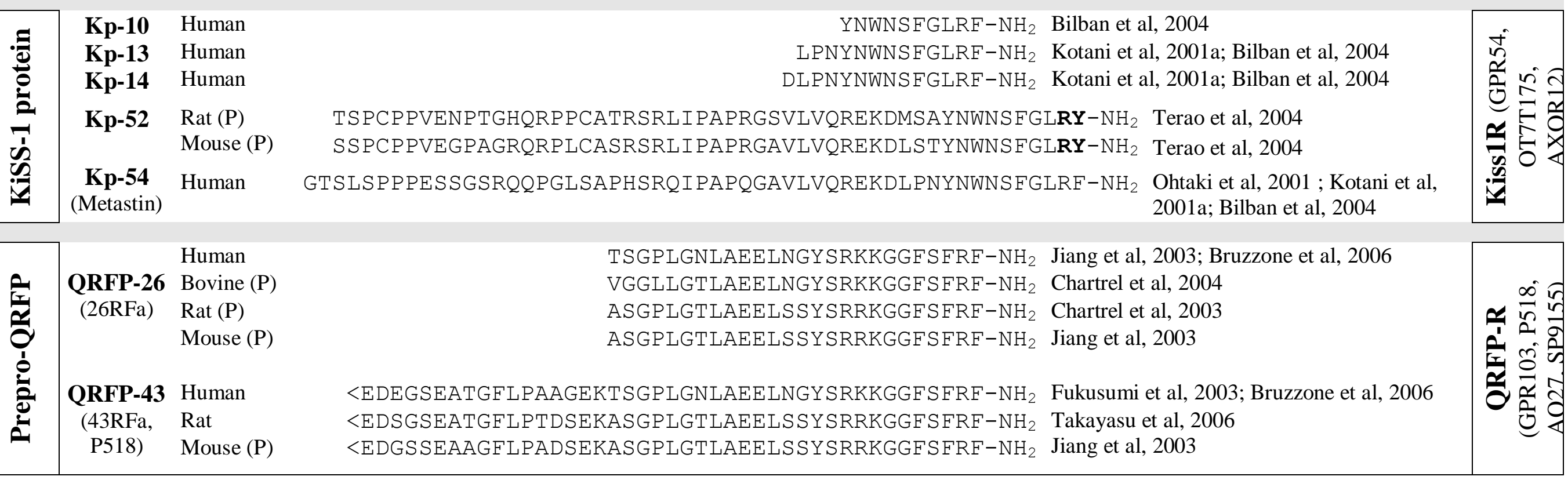

Mammalian RF-amide precursors (left boxes), RF-amide peptides (central panels) and RF-amide receptors (right boxes) of human, bovine and rodent origin are liste according to current nomenclature. Previous or alternative denominations are given under parentheses. RF-amide peptide sequences are

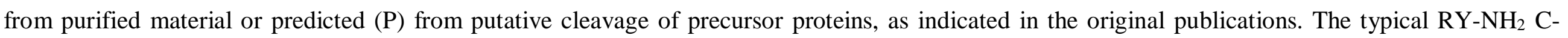
terminal sequence of mouse and rat kisspeptins is indicated in bold character. 
Table 2. Binding affinities of human NPFF1 and NPFF2 receptors for various RF-amide peptides as afforded from binding studies using tritiated and iodinated radioligands.

\begin{tabular}{|c|c|c|c|c|c|c|c|c|c|c|}
\hline \multirow{2}{*}{\multicolumn{2}{|c|}{ Tracer }} & \multicolumn{4}{|c|}{ NPFF1R } & \multicolumn{5}{|c|}{ NPFF2R } \\
\hline & & ${ }^{125}$ I-DMe & ${ }^{125}$ I-YVP & ${ }^{3}$ H-RFRP-3 & ${ }^{3}$ H-FFRF & ${ }^{125} \mathrm{I}-\mathrm{Y} 8 \mathrm{Fa}$ & ${ }^{125} \mathrm{I}-\mathrm{DMe}$ & ${ }^{125} \mathrm{I}-\mathrm{EYF}$ & ${ }^{3}$ H-EYF & ${ }^{3}$ H-FFRF \\
\hline Tracer $K_{d}(n M)$ & & $1.13^{\mathrm{a}}$ & $\begin{array}{l}0.18^{\mathrm{b}}-0.14^{\mathrm{c}} \\
0.27^{\mathrm{d}}-0.19^{\mathrm{e}}\end{array}$ & $2.65^{\mathrm{f}}$ & $1^{\mathrm{g}}$ & $0.24^{\mathrm{d}}-0.5^{\mathrm{h}}$ & $0.37^{\mathrm{a}}-0.1^{\mathrm{i}}$ & $0.06^{\mathrm{b}, \mathrm{k}}-0.07^{\mathrm{c}}$ & $0.54^{\mathrm{f}}$ & $0.2^{\mathrm{g}}$ \\
\hline \multicolumn{11}{|c|}{ Pro-NPFF $F_{A}$-derived peptides (Length, aa; $\mathrm{K}_{\mathrm{i}}$ values, $\mathrm{nM}$ ) } \\
\hline NPFF & 8 aа & & $2.5^{\mathrm{b}}-2.8^{\mathrm{c}}-2.4^{\mathrm{d}}$ & $20^{\mathrm{f}}$ & $0.3^{\mathrm{g}}$ & $1^{\mathrm{d}}-1.14^{\mathrm{h}}$ & $5.2^{\mathrm{i}}$ & $0.21^{\mathrm{b}, \mathrm{c}}-0.3^{\mathrm{k}}$ & $0.63^{\mathrm{f}}$ & $0.8^{\mathrm{g}}$ \\
\hline I-Y-NPFF (I-Y8Fa) & 8 aа & & $0.68^{\mathrm{d}}$ & & & & & & & \\
\hline 1DMe-NPFF & 8 aа & $8^{\mathrm{a}}$ & $2^{\mathrm{b}}-1.1^{\mathrm{c}}$ & $4.2^{\mathrm{f}}$ & & & $3.2^{\mathrm{a}}-5.3^{\mathrm{i}}$ & $0.18^{\mathrm{b}, \mathrm{c}}-0.31^{\mathrm{k}}$ & $0.38^{\mathrm{f}}$ & \\
\hline hSQA-NPFF & 11 aa & & $7^{\mathrm{b}}-4.1^{\mathrm{c}}-4.2^{\mathrm{d}}$ & $25^{\mathrm{f}}$ & $0.37^{\mathrm{g}}$ & $0.34^{\mathrm{d}}-0.11^{\mathrm{h}}$ & & $0.3^{\mathrm{b}, \mathrm{k}}-0.16^{\mathrm{c}}$ & $0.14^{\mathrm{f}}$ & $0.1^{\mathrm{g}}$ \\
\hline mSPA-NPFF & 11 aa & & $2.6^{\mathrm{b}}-4.0^{\mathrm{d}}$ & $7.6^{\mathrm{f}}$ & & $0.36^{\mathrm{d}}-$ & & $0.07^{\mathrm{b}}-0.05^{\mathrm{c}}$ & $0.04^{\mathrm{f}}$ & \\
\hline rNPA-NPFF & 11 aa & & $15^{\mathrm{b}}-3.4^{\mathrm{c}}$ & & & & & $0.11^{\mathrm{b}}-0.03^{\mathrm{c}}$ & & \\
\hline $\mathrm{r}, \mathrm{m}, \mathrm{h}$ NPAF (NPSF) & 8 aа & & $48^{b}-32^{c}$ & $109^{f}$ & & & $37^{\mathrm{i}}$ & $12^{\mathrm{b}, \mathrm{k}}-20^{\mathrm{c}}$ & $19^{\mathrm{f}}$ & \\
\hline mQFW-NPAF & 11 aа & & $8.8^{\mathrm{b}}$ & $37^{\mathrm{f}}$ & & & & $0.35^{\mathrm{b}, \mathrm{k}}-0.19^{\mathrm{c}}$ & $0.29^{f}$ & \\
\hline rEFW-NPAF & 11 aa & & $45^{\mathrm{b}}-21^{\mathrm{c}}$ & & & & & $0.21^{\mathrm{c}}$ & & \\
\hline EYW-NPAF (EYF) & 11 aa & & $15^{\mathrm{b}}-18^{\mathrm{c}}$ & & & & & $0.32^{\mathrm{b}, \mathrm{k}}-0.24^{\mathrm{c}}$ & & \\
\hline hNPAF & 18 aа & & $12^{b}-13^{c}$ & & $3.1^{\mathrm{g}}$ & $0.64^{\mathrm{h}}$ & & $0.22^{\mathrm{b}, \mathrm{k}}-0.14^{\mathrm{c}}$ & & $0.9^{\mathrm{g}}$ \\
\hline bNPAF & 18 aа & $63^{\mathrm{a}}$ & $10^{\mathrm{b}}$ & & & $1.8^{\mathrm{h}}$ & $1.3^{\mathrm{a}}$ & $0.16^{\mathrm{c}}$ & & \\
\hline \multicolumn{11}{|c|}{ Pro-NPFF $F_{B}$-derived peptides (Length, aa; $\mathrm{K}_{\mathrm{i}}$ values, $\mathrm{nM}$ ) } \\
\hline hRFRP-1 & $12 \mathrm{aa}$ & & $0.4^{\mathrm{j}}-1.3^{\mathrm{c}}-2.7^{\mathrm{d}}$ & & & $3.8^{\mathrm{d}}$ & $15^{\mathrm{i}}$ & $3.9^{\mathrm{c}}-2.8^{\mathrm{j}}$ & & \\
\hline hRFRP-1 & 37 aа & & $15^{\mathrm{d}}$ & & $0.58^{\mathrm{g}}$ & $17^{\mathrm{d}}$ & & & & $15^{\mathrm{g}}$ \\
\hline hRFRP-3 & 8 aа & & $0.5^{\mathrm{b}}-0.6^{\mathrm{c}}-1.2^{\mathrm{d}}$ & $3.4^{\mathrm{f}}$ & $0.2^{\mathrm{g}}$ & $150^{\mathrm{d}}$ & $55^{\mathrm{i}}$ & $17.4^{\mathrm{b}, \mathrm{c}}-23^{\mathrm{j}}$ & $32^{\mathrm{f}}$ & $67^{g}$ \\
\hline hY-RFRP-3 (YVP) & 9 aа & & $0.4^{b}-0.7^{c}$ & & & & & $9^{b, c}$ & & \\
\hline bRFRP-3 & 28 aа & & $1.6^{\mathrm{d}}$ & & & $7^{\mathrm{d}}$ & & & & \\
\hline \multicolumn{11}{|c|}{ Miscellaneous peptides (Length, aa; $\mathrm{K}_{\mathrm{i}}$ values, $\mathrm{nM}$ ) } \\
\hline FMRF amide & 4 aa & $0.8^{\mathrm{a}}$ & $2.1^{\mathrm{b}}-1.8^{\mathrm{c}}$ & & & & $4^{\mathrm{a}}-13^{\mathrm{i}}$ & $6.6^{\mathrm{b}, \mathrm{c}}-7^{\mathrm{j}}-10^{\mathrm{k}}$ & & \\
\hline PQRF amide & 4 aa & & $23^{\mathrm{b}}-8.7^{\mathrm{c}}-15^{\mathrm{d}}$ & & & $26^{\mathrm{d}}$ & & $6.8^{\mathrm{b}, \mathrm{c}}$ & & \\
\hline Frog Pancreatic Peptide & 36 aа & $1260^{\mathrm{a}}$ & $4470^{\mathrm{b}}->1000^{\mathrm{c}}$ & & & & $40^{\mathrm{a}}$ & $7^{\mathrm{c}}-5.1^{\mathrm{j}}$ & & \\
\hline
\end{tabular}

Human (h), bovine (b), rat (r) and mouse (m) RF-amide peptides are listed according to their precursor origin along with their amino-acid length.

$\mathrm{K}_{\mathrm{i}}$-values are from competition binding studies performed on NPFF1R- or NPFF2R-transfected cell lines as reported in the associated references.

Notes to Table 2 : 
a) Bonini et al, (2000); b) Gouarderes et al, (2002); c) Mollereau et al, (2002); d) Yoshida et al, (2003); e) Hinuma et al, (2000); f) Talmont et al, (2009);

g) Elhabazi et al, (2013); h) Elshourbagy et al, (2000); i) Engstrom et al, (2003); j) Gourderes et al, (2007); k) Kotani et al, (2001b). 
Table 3. Summary of binding affinities and functional potencies of various RF-amide peptides at the five human RF-amide receptors

\begin{tabular}{|c|c|c|c|c|c|c|c|c|c|c|}
\hline \multirow[b]{3}{*}{ Peptide } & \multicolumn{2}{|c|}{ NPFF1R } & \multicolumn{2}{|c|}{ NPFF2R } & \multicolumn{2}{|c|}{ PrRP-R } & \multicolumn{2}{|c|}{ Kiss1R } & \multicolumn{2}{|c|}{ QRFP-R } \\
\hline & $\begin{array}{l}\text { Binding } \\
\text { affinity }\end{array}$ & $\begin{array}{c}\text { cAMP } \\
\text { response }\end{array}$ & $\begin{array}{l}\text { Binding } \\
\text { affinity }\end{array}$ & $\begin{array}{c}\text { cAMP } \\
\text { response }\end{array}$ & $\begin{array}{l}\text { Binding } \\
\text { affinity }\end{array}$ & $\begin{array}{l}\text { Calcium } \\
\text { response }\end{array}$ & $\begin{array}{l}\text { Binding } \\
\text { affinity }\end{array}$ & $\begin{array}{l}\text { Calcium } \\
\text { response }\end{array}$ & $\begin{array}{l}\text { Binding } \\
\text { affinity }\end{array}$ & $\begin{array}{l}\text { Calcium } \\
\text { response }\end{array}$ \\
\hline & $\mathrm{K}_{\mathrm{i}}, \mathrm{nM}$ & $\mathrm{IC}_{50}, \mathrm{nM}$ & $\mathrm{K}_{\mathrm{i}}, \mathrm{nM}$ & $\mathrm{IC}_{50}, \mathrm{nM}$ & $\mathrm{K}_{\mathrm{i}}, \mathrm{nM}$ & $\mathrm{EC}_{50} \mathrm{nM}$ & $\mathrm{K}_{\mathrm{i}}, \mathrm{nM}$ & $\mathrm{EC}_{50} \mathrm{nM}$ & $\mathrm{K}_{\mathrm{i}}, \mathrm{nM}$ & $\mathrm{EC}_{50}, \mathrm{nM}$ \\
\hline NPFF & $2.5^{*}$ & $31-510^{\mathrm{a}, \mathrm{d}-\mathrm{g}}$ & $0.25 *$ & $0.6-7^{\mathrm{a}, \mathrm{d}, \mathrm{f}, \mathrm{g}, \mathrm{i}}$ & $>20,000^{\mathrm{a}, \mathrm{h}}$ & $>10,000^{\mathrm{a}}$ & $>20,000^{a}$ & $>10,000^{\mathrm{a}}$ & $>20,000^{a}$ & $>10,000^{\mathrm{a}}$ \\
\hline 1DMe-NPFF & $1.5^{*}$ & $71^{f}$ & $0.25^{*}$ & $2.7^{\mathrm{f}}$ & & & & & & \\
\hline hSQA-NPFF & $5^{*}$ & $47-153^{\mathrm{d}, \mathrm{f}}$ & $0.23 *$ & $0.25-0.9^{\mathrm{d}, \mathrm{f}, \mathrm{i}, \mathrm{r}}$ & $>20,000^{\mathrm{a}}$ & $>10,000^{\mathrm{a}}$ & $>20,000^{\mathrm{a}}$ & $>10,000^{\mathrm{a}}$ & $>20,000^{\mathrm{a}}$ & $>10,000^{\mathrm{a}}$ \\
\hline $\mathrm{r}, \mathrm{m}, \mathrm{h}$ NPSF & $40^{*}$ & $876^{f}$ & $16^{*}$ & $222^{\mathrm{f}}$ & & & & & & \\
\hline mQFW-NPAF & $8.8^{*}$ & & 0.27 & $1.5^{\mathrm{f}}$ & & & & & & \\
\hline hNPAF (18a) & $12.5^{*}$ & $324-1350^{a, f}$ & $0.18 *$ & $0.18-3^{\mathrm{a}, \mathrm{f}, \mathrm{i}}$ & $>20,000^{\mathrm{a}}$ & $>10,000^{\mathrm{a}}$ & $>20,000^{a}$ & $>10,000^{\mathrm{a}}$ & $>20,000^{a}$ & $>10,000^{\mathrm{a}}$ \\
\hline bNPAF (18a) & & & $0.16^{*}$ & $0.4-1.5^{f, i}$ & & & & & & \\
\hline hRFRP-1 (12a) & $1.5^{*}$ & $4.5-9.6^{\mathrm{d}-\mathrm{f}}$ & $3.3^{*}$ & $21-330^{d, f}$ & $>100,000^{h}$ & & & & & \\
\hline hRFRP-1 (37a) & $15^{*}$ & $12-20^{\mathrm{a}, \mathrm{d}, \mathrm{e}}$ & & $150-470^{\mathrm{a}, \mathrm{d}}$ & $>20,000^{\mathrm{a}}$ & $>10,000^{\mathrm{a}}$ & $>20,000^{\mathrm{a}}$ & $>10,000^{\mathrm{a}}$ & $>20,000^{a}$ & $>10,000^{\mathrm{a}}$ \\
\hline hRFRP-3 (8a) & $0.8^{*}$ & $3-12^{a, d-g}$ & $20 *$ & $74-1,000^{\mathrm{a}, \mathrm{d}, \mathrm{f}, \mathrm{g}}$ & $>20,000^{\mathrm{a}, \mathrm{h}}$ & $>10,000^{\mathrm{a}}$ & $>20,000^{a}$ & $>10,000^{\mathrm{a}}$ & $>20,000^{\mathrm{a}}$ & $>10,000^{\mathrm{a}}$ \\
\hline hPrRP-20 & $4.7^{\mathrm{a}}$ & $9280^{a}$ & $0.7-23^{\mathrm{a}, \mathrm{h}}$ & $466^{\mathrm{a}}$ & $0.06-1^{\mathrm{a}, \mathrm{h}, \mathrm{j}, \mathrm{k}}$ & $0.2-1^{\mathrm{a}, \mathrm{j}, \mathrm{k}}$ & $>20,000^{\mathrm{a}}$ & $>10,000^{\mathrm{a}}$ & $>20,000^{\mathrm{a}}$ & $>10,000^{\mathrm{a}}$ \\
\hline hPrRP-31 & $11-45^{\mathrm{a}, \mathrm{b}}$ & $>10,000^{a}$ & $0.4-19^{\mathrm{a}, \mathrm{b}, \mathrm{h}}$ & $240^{\mathrm{a}}$ & $0.07-1^{\mathrm{a}, \mathrm{h}, \mathrm{j}, \mathrm{k}}$ & $0.3-1.5^{\mathrm{a}, \mathrm{k}, \mathrm{l}}$ & $>20,000^{\mathrm{a}}$ & $>10,000^{\mathrm{a}}$ & $>20,000^{a}$ & $>10,000^{\mathrm{a}}$ \\
\hline $\mathrm{Kp}-10$ & $0.5-4.7^{\mathrm{a}, \mathrm{c}}$ & $380-10,000^{\mathrm{a}, \mathrm{c}}$ & $1.6-76^{\mathrm{a}, \mathrm{c}}$ & $292-1,000^{\mathrm{a}, \mathrm{c}}$ & $9,200^{\mathrm{a}}$ & $>10,000^{\mathrm{a}}$ & $0.04-2.3^{\mathrm{a}, \mathrm{m}, \mathrm{n}}$ & $0.1^{\mathrm{a}, \mathrm{m}}$ & $8,020^{\mathrm{a}}$ & $>10,000^{\mathrm{a}}$ \\
\hline $\mathrm{Kp}-13$ & $0.7^{\mathrm{a}}$ & $830^{a}$ & $1.4^{\mathrm{a}}$ & $475^{\mathrm{a}}$ & $5,800^{\mathrm{a}}$ & $>10,000^{\mathrm{a}}$ & $0.9-4.2^{\mathrm{a}, \mathrm{n}}$ & $0.17^{\mathrm{a}}$ & $>20,000^{\mathrm{a}}$ & $>10,000^{\mathrm{a}}$ \\
\hline $\mathrm{Kp}-54$ & $0.5-15^{\mathrm{a}, \mathrm{c}}$ & $>10,000^{\mathrm{a}, \mathrm{c}}$ & $10-400^{\mathrm{a}, \mathrm{c}}$ & $>5,000^{\mathrm{a}, \mathrm{c}}$ & $>20,000^{\mathrm{a}}$ & $>10,000^{\mathrm{a}}$ & $0.3-3.7^{\mathrm{a}, \mathrm{m}, \mathrm{n}}$ & $0.3-0.4^{\mathrm{a}, \mathrm{m}}$ & $>20,000^{a}$ & $>10,000^{\mathrm{a}}$ \\
\hline hQRFP-26 & $24-38^{a, b}$ & $>10,000^{\mathrm{a}}$ & $3-10^{a, b}$ & $>10,000^{\mathrm{a}}$ & $10,500^{\mathrm{a}}$ & $>10,000^{\mathrm{a}}$ & $>20,000^{a}$ & $>10,000^{\mathrm{a}}$ & $3.2-21^{\mathrm{a}, \mathrm{o}}$ & $0.2-7^{\mathrm{a}, \mathrm{q}}$ \\
\hline hQRFP-43 & $84-330^{a, b}$ & $>10,000^{\mathrm{a}}$ & $53-131^{\mathrm{a}, \mathrm{b}}$ & $>10,000^{\mathrm{a}}$ & $>20,000^{a}$ & $>10,000^{\mathrm{a}}$ & $>20,000^{a}$ & $>10,000^{\mathrm{a}}$ & $0.1-8^{\mathrm{a}, \mathrm{o}, \mathrm{p}}$ & $0.3-23^{\mathrm{a}, \mathrm{p}}$ \\
\hline
\end{tabular}

RF-amide peptides of rat (r), mouse (m), bovine (b) or human (h) origin have been considered, with aminoacid lengths indicated under parentheses when needed. Reported affinity constants are mean $\mathrm{K}_{\mathrm{i}}$ values taken from Table 2, using [ $\left.{ }^{125} \mathrm{I}\right]-\mathrm{YVP}$ (hNPFF1) or [ $\left.{ }^{125} \mathrm{I}\right]-\mathrm{EYF}$ (hNPFF2) as the radioligand. Otherwise, affinity $\left(\mathrm{K}_{\mathrm{i}}\right)$ and potency $\left(\mathrm{EC}_{50}\right.$ and $\left.\mathrm{IC}_{50}\right)$ values are min-max for the set of values selected from the literature.

Notes to Table 3 : 
a) Elhabazi et al, (2013); b) Gouarderes et al, (2007); c) Oishi et al, (2011); d) Yoshida et al, (2003); e) Fukusumi et al, (2001); f) Mollereau et al, (2002); g) Liu et al, (2001); h) Engstrom et al, (2003); i) Elshourbagy et al, (2000); j) Roland et al, (1999); k) Langmead et al, (2000); 1) Wang et al, (2012); m) Ohtaki et al, (2001); n) Kotani et al, (2001a); o) Fukusumi et al, (2003); p) Takayasu et al, (2006); q) Jiang et al, (2003); ); r) Kotani et al, (2001b). 
Table 4. Binding affinities of NPFF-related peptides for endogenous RF-amide receptors expressed in the dorsal horn of rat spinal cord.

\begin{tabular}{|c|c|c|c|c|c|c|c|c|c|c|c|}
\hline \multirow{3}{*}{$\begin{array}{c}\text { NPFF } \\
\text { derivatives }\end{array}$} & \multirow{2}{*}{\multicolumn{9}{|c|}{ Sequence }} & \multicolumn{2}{|c|}{$K_{i}$ values $(\mathbf{n M})$} \\
\hline & & & & & & & & & & \multirow[t]{2}{*}[{}^{125}\mathrm{I}]{$\mathrm{Tyr}^{1}-\mathrm{NPFF}^{\mathrm{a}}$} & \multirow[t]{2}{*}[{}^{125}\mathrm{I}]{$-1 \mathrm{DM} \mathrm{e}^{\mathrm{b}}$} \\
\hline & 1 & 2 & 3 & 4 & 5 & 6 & 7 & 8 & & & \\
\hline NPFF & $\mathrm{F}$ & $\mathrm{L}$ & F & Q & $\mathrm{P}$ & $Q$ & $\mathrm{R}$ & F & $-\mathrm{NH}_{2}$ & $0.21 \pm 0.03$ & $0.34 \pm 0.07$ \\
\hline NPFF-OH & F & $\mathrm{L}$ & F & Q & $\mathrm{P}$ & $Q$ & $\mathrm{R}$ & F & $-\mathrm{OH}$ & & $5178 \pm 2195$ \\
\hline $\operatorname{NPFF}(2-8)$ & & $\mathrm{L}$ & F & Q & $\mathrm{P}$ & $Q$ & $\mathrm{R}$ & F & $-\mathrm{NH}_{2}$ & $0.20 \pm 0.02$ & \\
\hline $\operatorname{NPFF}(3-8)$ & & & F & Q & $\mathrm{P}$ & $Q$ & $\mathrm{R}$ & F & $-\mathrm{NH}_{2}$ & $0.43 \pm 0.06$ & \\
\hline NPFF(4-8) & & & & Q & $\mathrm{P}$ & $Q$ & $\mathrm{R}$ & F & $-\mathrm{NH}_{2}$ & $20.9 \pm 3.1$ & \\
\hline $\operatorname{NPFF}(5-8)$ & & & & & $\mathrm{P}$ & $Q$ & $\mathrm{R}$ & F & $-\mathrm{NH}_{2}$ & $15.5 \pm 2.3$ & \\
\hline $\operatorname{NPFF}(6-8)$ & & & & & & $Q$ & $\mathrm{R}$ & F & $-\mathrm{NH}_{2}$ & $300 \pm 45$ & \\
\hline$\left[\mathrm{Tyr}^{8}\right] \mathrm{NPFF}$ & $\mathrm{F}$ & $\mathrm{L}$ & $\mathrm{F}$ & $Q$ & $\mathrm{P}$ & $Q$ & $\mathrm{R}$ & $\mathbf{Y}$ & $-\mathrm{NH}_{2}$ & & $34 \pm 10.2$ \\
\hline$\left[\mathrm{Ala}^{8}\right] \mathrm{NPFF}$ & $\mathrm{F}$ & $\mathrm{L}$ & $\mathrm{F}$ & $Q$ & $\mathrm{P}$ & $Q$ & $\mathrm{R}$ & A & $-\mathrm{NH}_{2}$ & & $312 \pm 73$ \\
\hline$\left[\mathrm{Lys}^{7}\right] \mathrm{NPFF}$ & $\mathrm{F}$ & $\mathrm{L}$ & $\mathrm{F}$ & $Q$ & $\mathrm{P}$ & $Q$ & $\mathrm{~K}$ & F & $-\mathrm{NH}_{2}$ & & $245 \pm 90$ \\
\hline$\left[\mathrm{Ala}^{7}\right] \mathrm{NPFF}$ & $\mathrm{F}$ & $\mathrm{L}$ & $\mathrm{F}$ & $Q$ & $\mathrm{P}$ & $Q$ & $A$ & F & $-\mathrm{NH}_{2}$ & & $2359 \pm 617$ \\
\hline$\left[\mathrm{Glu}^{6}\right] \mathrm{NPFF}$ & $\mathrm{F}$ & $\mathrm{L}$ & $\mathrm{F}$ & $Q$ & $\mathrm{P}$ & $\mathrm{D}$ & $\mathrm{R}$ & F & $-\mathrm{NH}_{2}$ & & $307 \pm 87$ \\
\hline$\left[\mathrm{Asn}^{6}\right] \mathrm{NPFF}$ & $\mathrm{F}$ & $\mathrm{L}$ & $\mathrm{F}$ & $Q$ & $\mathrm{P}$ & $\mathbf{N}$ & $\mathrm{R}$ & F & $-\mathrm{NH}_{2}$ & & $7.3 \pm 1.7$ \\
\hline$\left[\mathrm{Gly}^{4}\right] \mathrm{NPFF}$ & $\mathrm{F}$ & $\mathrm{L}$ & $\mathrm{F}$ & $\mathbf{G}$ & $\mathrm{P}$ & $Q$ & $\mathrm{R}$ & F & $-\mathrm{NH}_{2}$ & & $10 . \pm 1.000$ \\
\hline$\left[\mathrm{Glu}^{4}\right] \mathrm{NPFF}$ & $\mathrm{F}$ & $\mathrm{L}$ & $\mathrm{F}$ & $D$ & $\mathrm{P}$ & $Q$ & $\mathrm{R}$ & F & $-\mathrm{NH}_{2}$ & & $1.15 \pm 0.26$ \\
\hline$\left[\mathrm{Asn}^{4}\right] \mathrm{NPFF}$ & $\mathrm{F}$ & $\mathrm{L}$ & $\mathrm{F}$ & $\mathbf{N}$ & $\mathrm{P}$ & $Q$ & $\mathrm{R}$ & F & $-\mathrm{NH}_{2}$ & & $2.34 \pm 1.09$ \\
\hline$\left[\mathrm{Tyr}^{3}\right] \mathrm{NPFF}$ & $\mathrm{F}$ & $\mathrm{L}$ & $Y$ & $Q$ & $\mathrm{P}$ & $Q$ & $\mathrm{R}$ & F & $-\mathrm{NH}_{2}$ & & $0.22 \pm 0.03$ \\
\hline
\end{tabular}

${ }^{a} \mathrm{~K}_{\mathrm{i}}$ values are from competition studies performed on rat spinal cord membrane preparations (Gicquel et al., 1994).

${ }^{\mathrm{b}} \mathrm{K}_{\mathrm{i}}$ values are from quantitative autoradiography performed on rat spinal cord sections (Mazarguil et al., 2001). 
Table 5. Comparison of potency $\left(\mathrm{EC}_{50}\right)$ and efficacy $\left(\mathrm{E}_{\max }\right)$ at human NPFF1 and NPFF2 receptors of NPFF analogs with variations in their RF-amide motif. ${ }^{\text {a }}$

\begin{tabular}{|c|c|c|c|c|c|}
\hline \multirow{3}{*}{$\begin{array}{c}\text { NPFF } \\
\text { derivatives }\end{array}$} & \multirow{3}{*}{$\begin{array}{l}\text { Sequence } \\
\mathrm{Q}-\mathrm{P}-\mathrm{Q}-\mathrm{Arg}-\mathrm{Ph} e-\mathrm{NH}_{2}\end{array}$} & \multicolumn{4}{|c|}{$\mathbf{E C}_{50}(\mathrm{nM}) ; \mathbf{E}_{\max }(\%)$} \\
\hline & & \multicolumn{2}{|c|}{ NPFF1R } & \multicolumn{2}{|c|}{ NPFF2R } \\
\hline & & 12 & $100 \%$ & 3 & $100 \%$ \\
\hline \multicolumn{6}{|c|}{ Modification of the phenylalanine at position 8} \\
\hline$\left[\mathrm{Ala}^{8}\right] \mathrm{NPFF}$ & $\mathrm{F}-\mathrm{L}-\mathrm{F}-\mathrm{Q}-\mathrm{P}-\mathrm{Q}-\mathrm{Arg}-\mathbf{A} \mathbf{l} \mathbf{a}-\mathrm{NH}_{2}$ & ND & - & ND & $(25 \%)$ \\
\hline$\left[\mathrm{Nle}^{8}\right] \mathrm{NPFF}$ & $\mathrm{F}-\mathrm{L}-\mathrm{F}-\mathrm{Q}-\mathrm{P}-\mathrm{Q}-\mathrm{Arg}-\mathrm{Nl} \mathbf{e}-\mathrm{NH}_{2}$ & 659 & $93 \%$ & 287 & $(83 \%$ \\
\hline$\left[\mathrm{Cha}^{8}\right] \mathrm{NPFF}$ & $\mathrm{F}-\mathrm{L}-\mathrm{F}-\mathrm{Q}-\mathrm{P}-\mathrm{Q}-\mathrm{Arg}-\mathrm{Ch} \mathbf{a}-\mathrm{NH}_{2}$ & 44 & $91 \%$ & 17 & $(98 \%)$ \\
\hline$\left[\mathrm{Tyr}^{8}\right] \mathrm{NPFF}$ & $\mathrm{F}-\mathrm{L}-\mathrm{F}-\mathrm{Q}-\mathrm{P}-\mathrm{Q}-\mathrm{Arg}-\mathrm{Ty} \mathbf{r}-\mathrm{NH}_{2}$ & 301 & $91 \%$ & 70 & $(83 \%)$ \\
\hline$\left[\operatorname{Trp}^{8}\right]$ NPFF & $\mathrm{F}-\mathrm{L}-\mathrm{F}-\mathrm{Q}-\mathrm{P}-\mathrm{Q}-\mathrm{Arg}-\mathbf{T r} \mathbf{p}-\mathrm{NH}_{2}$ & 3410 & $55 \%$ & 205 & $(99 \%)$ \\
\hline$\left[\mathrm{His}^{8}\right] \mathrm{NPFF}$ & $\mathrm{F}-\mathrm{L}-\mathrm{F}-\mathrm{Q}-\mathrm{P}-\mathrm{Q}-\mathrm{Arg}-\mathrm{His}-\mathrm{NH}_{2}$ & 2180 & $29 \%$ & 2750 & $67 \%$ \\
\hline$\left[\mathrm{D}-\mathrm{Phe}^{8}\right] \mathrm{NPFF}$ & $\mathrm{F}-\mathrm{L}-\mathrm{F}-\mathrm{Q}-\mathrm{P}-\mathrm{Q}-\mathrm{Arg}-\mathrm{Ph} \mathbf{e}-\mathrm{NH}_{2}$ & 200 & $84 \%$ & 132 & $115 \%$ \\
\hline$\left[\mathrm{Hph}^{8}\right] \mathrm{NPFF}$ & $\mathrm{F}-\mathrm{L}-\mathrm{F}-\mathrm{Q}-\mathrm{P}-\mathrm{Q}-\mathrm{Arg}-\mathrm{Hph}-\mathrm{NH}_{2}$ & 1760 & $61 \%$ & 1331 & $98 \%$ \\
\hline$\left[\mathrm{Phg}^{8}\right] \mathrm{NPFF}$ & $\mathrm{F}-\mathrm{L}-\mathrm{F}-\mathrm{Q}-\mathrm{P}-\mathrm{Q}-\mathrm{Arg}-\mathbf{P h} \mathbf{g}-\mathrm{NH}_{2}$ & 1780 & $29 \%$ & 1070 & $98 \%$ \\
\hline \multicolumn{6}{|c|}{ Modification of the arginine at position 7} \\
\hline$\left[\mathrm{Ala}^{7}\right] \mathrm{NPFF}$ & $\mathrm{F}-\mathrm{L}-\mathrm{F}-\mathrm{Q}-\mathrm{P}-\mathrm{Q}-\mathrm{Al} \mathbf{a}-\mathrm{Ph} e-\mathrm{NH}_{2}$ & 7610 & $26 \%$ & 1228 & $(60 \%)$ \\
\hline$\left[\mathrm{Cit}^{7}\right] \mathrm{NPFF}$ & $\mathrm{F}-\mathrm{L}-\mathrm{F}-\mathrm{Q}-\mathrm{P}-\mathrm{Q}-\mathrm{Cit}-\mathrm{Phe}-\mathrm{NH}_{2}$ & 3170 & $66 \%$ & 2469 & $(59 \%)$ \\
\hline$\left[\mathrm{Lys}^{7}\right] \mathrm{NPFF}$ & $\mathrm{F}-\mathrm{L}-\mathrm{F}-\mathrm{Q}-\mathrm{P}-\mathrm{Q}-\mathrm{Ly} \mathbf{s}-\mathrm{Ph} e-\mathrm{NH}_{2}$ & 1290 & $83 \%$ & 565 & $(81 \%)$ \\
\hline$\left[\mathrm{Orn}^{7}\right] \mathrm{NPFF}$ & $\mathrm{F}-\mathrm{L}-\mathrm{F}-\mathrm{Q}-\mathrm{P}-\mathrm{Q}-\mathrm{Orn}-\mathrm{Phe}-\mathrm{NH}_{2}$ & 3510 & $57 \%$ & 1692 & $74 \%$ \\
\hline$\left[\mathrm{Agb}^{7}\right] \mathrm{NPFF}$ & $\mathrm{F}-\mathrm{L}-\mathrm{F}-\mathrm{Q}-\mathrm{P}-\mathrm{Q}-\mathbf{A g} \mathbf{g}-\mathrm{Ph} e-\mathrm{NH}_{2}$ & 1390 & $31 \%$ & 1219 & $96 \%$ \\
\hline$\left[\mathrm{Agp}^{7}\right] \mathrm{NPFF}$ & $\mathrm{F}-\mathrm{L}-\mathrm{F}-\mathrm{Q}-\mathrm{P}-\mathrm{Q}-\mathbf{A g p}-\mathrm{Phe}-\mathrm{NH}_{2}$ & 3200 & $17 \%$ & 1524 & $54 \%$ \\
\hline
\end{tabular}

${ }^{a}$ Data are taken from Findeisen et al., 2011b.

Values are from dose-response curves using an inositol phosphate accumulation assay on COS-7 cells transfected with human NPFF receptors and a chimeric $\mathrm{G \alpha}_{\mathrm{q}}$ protein. EC50 values refer to agonist concentrations that evoked a half-maximal response. $\mathrm{E}_{\max }$ values were defined at the highest agonist concentration tested, usually at $100 \mu \mathrm{M}$ or at $10 \mu \mathrm{M}$ (when indicated under parentheses). ND: the $\mathrm{EC}_{50}$ value was not determined as full receptor activation could not be ascertained at the agonist concentration which was tested $(10 \mu \mathrm{M})$. 
Table 6. Binding affinity and functional potency of PrRP-(19-31) peptide analogues at PrRP receptors. $^{\text {a }}$

\begin{tabular}{|c|c|c|c|c|c|c|c|c|c|c|c|c|c|c|c|c|}
\hline \multirow{2}{*}{$\begin{array}{l}\text { PrRP-related } \\
\text { peptides }\end{array}$} & \multirow{2}{*}{\multicolumn{14}{|c|}{ Sequence }} & \multicolumn{2}{|c|}{ PrRP-R } \\
\hline & & & & & & & & & & & & & & & $\mathbf{K}_{\mathbf{i}}(\mu \mathrm{M})$ & $\mathbf{E C}_{50}(\mu \mathrm{M})$ \\
\hline (19-31)PrRP & W & $\mathrm{Y}$ & A & S & $\mathrm{R}$ & G & I & $\mathrm{R}$ & $P$ & V & G & $\mathrm{R}$ & $\mathrm{F}$ & $-\mathrm{NH}_{2}$ & 0.0053 & 0.02 \\
\hline (19-31)PrRP acid & W & $\mathrm{Y}$ & A & S & $\mathrm{R}$ & G & I & $\mathrm{R}$ & $\mathrm{P}$ & $\mathrm{V}$ & G & $\mathrm{R}$ & $\mathrm{F}$ & $-\mathrm{OH}$ & 5.0000 & - \\
\hline$\left[\operatorname{Trp}^{31}\right](19-31) \operatorname{PrRP}$ & $W$ & $Y$ & A & S & $\mathrm{R}$ & G & I & $\mathrm{R}$ & $\mathrm{P}$ & $\mathrm{V}$ & G & $\mathrm{R}$ & W & $-\mathrm{NH}_{2}$ & 0.0245 & 0.05 \\
\hline$\left[\mathrm{Nle}^{31}\right](19-31) \operatorname{PrRP}$ & $W$ & $\mathrm{Y}$ & A & S & $\mathrm{R}$ & G & I & $\mathrm{R}$ & $\mathrm{P}$ & $\mathrm{V}$ & G & $\mathrm{R}$ & Nle & $-\mathrm{NH}_{2}$ & 0.0195 & 0.05 \\
\hline$\left[\mathrm{Leu}^{31}\right](19-31) \operatorname{PrRP}$ & W & $Y$ & A & S & $\mathrm{R}$ & G & I & $\mathrm{R}$ & $\mathrm{P}$ & $\mathrm{V}$ & G & $\mathrm{R}$ & $\mathrm{I}$ & $-\mathrm{NH}_{2}$ & 0.395 & 2.06 \\
\hline$\left[\mathrm{Gln}^{31}\right](19-31) \operatorname{PrRP}$ & $W$ & $\mathrm{Y}$ & A & S & $\mathrm{R}$ & G & I & $\mathrm{R}$ & $\mathrm{P}$ & $\mathrm{V}$ & G & $\mathrm{R}$ & $Q$ & $-\mathrm{NH}_{2}$ & 0.0635 & 0.17 \\
\hline$\left[\mathrm{Met}^{31}\right](19-31) \operatorname{PrRP}$ & $\mathrm{W}$ & Y & A & S & $\mathrm{R}$ & G & I & $\mathrm{R}$ & $\mathrm{P}$ & $\mathrm{V}$ & G & $\mathrm{R}$ & $\tilde{M}$ & $-\mathrm{NH}_{2}$ & 0.0955 & 0.35 \\
\hline$\left[\right.$ Lys $\left.^{30}\right](19-31) \operatorname{PrRP}$ & $W$ & $\mathrm{Y}$ & A & S & $\mathrm{R}$ & G & I & $\mathrm{R}$ & $\mathrm{P}$ & $\mathrm{V}$ & G & $\mathrm{K}$ & $\mathrm{F}$ & $-\mathrm{NH}_{2}$ & - & Inactive \\
\hline$\left[\right.$ Pro $\left.^{30}\right](19-31) \operatorname{PrRP}$ & $W$ & $\mathrm{Y}$ & A & S & $\mathrm{R}$ & G & I & $\mathrm{R}$ & $\mathrm{P}$ & $\mathrm{V}$ & G & $\mathbf{P}$ & $\mathrm{F}$ & $-\mathrm{NH}_{2}$ & - & Inactive \\
\hline$\left[\mathrm{Ala}^{29}\right](19-31) \operatorname{PrRP}$ & $W$ & $\mathrm{Y}$ & A & S & $\mathrm{R}$ & G & I & $\mathrm{R}$ & $\mathrm{P}$ & $\mathrm{V}$ & A & $\mathrm{R}$ & $\mathrm{F}$ & $-\mathrm{NH}_{2}$ & 0.0945 & 0.24 \\
\hline$\left[\mathrm{Phg}^{28}\right](19-31) \operatorname{PrRP}$ & W & $\mathrm{Y}$ & A & S & $\mathrm{R}$ & G & I & $\mathrm{R}$ & $\mathrm{P}$ & Phg & G & $\mathrm{R}$ & $\mathrm{F}$ & $-\mathrm{NH}_{2}$ & 0.0047 & 0.01 \\
\hline$\left[\mathrm{Ala}^{27}\right](19-31) \operatorname{PrRP}$ & $W$ & $Y$ & A & S & $\mathrm{R}$ & G & I & $\mathrm{R}$ & A & $\mathrm{V}$ & G & $\mathrm{R}$ & $\mathrm{F}$ & $-\mathrm{NH}_{2}$ & 0.1870 & nd \\
\hline$\left[\mathrm{Ala}^{26}\right](19-31) \operatorname{PrRP}$ & W & $\mathrm{Y}$ & A & S & $\mathrm{R}$ & G & I & $\mathrm{K}$ & $\mathrm{P}$ & $\mathrm{V}$ & G & $\mathrm{R}$ & $\mathrm{F}$ & $-\mathrm{NH}_{2}$ & 0.0220 & 0.12 \\
\hline$\left[\mathrm{Ala}^{25}\right](19-31) \operatorname{PrRP}$ & $W$ & $Y$ & A & S & $\mathrm{R}$ & G & A & $\mathrm{R}$ & $\mathrm{P}$ & $\mathrm{V}$ & G & $\mathrm{R}$ & $\mathrm{F}$ & $-\mathrm{NH}_{2}$ & 0.0505 & 0.13 \\
\hline$\left[\mathrm{Phg}^{25}\right](19-31) \operatorname{PrRP}$ & W & $\mathrm{Y}$ & A & S & $\mathrm{R}$ & G & Phg & $\mathrm{R}$ & $\mathrm{P}$ & V & G & $\mathrm{R}$ & $\mathrm{F}$ & $-\mathrm{NH}_{2}$ & 0.0082 & 0.01 \\
\hline [Ala $\left.{ }^{24}\right](19-31) \operatorname{PrRP}$ & $W$ & $Y$ & A & S & $\mathrm{R}$ & $\mathbf{A}$ & $I$ & $\mathrm{R}$ & $\mathrm{P}$ & $\mathrm{V}$ & G & $\mathrm{R}$ & $\mathrm{F}$ & $-\mathrm{NH}_{2}$ & 0.0115 & 0.01 \\
\hline$\left[\mathrm{Pro}^{23}\right](19-31) \operatorname{PrRP}$ & W & $\mathrm{Y}$ & A & S & $\mathbf{P}$ & G & I & $\mathrm{R}$ & $\mathrm{P}$ & $\mathrm{V}$ & G & $\mathrm{R}$ & $\mathrm{F}$ & $-\mathrm{NH}_{2}$ & 0.0115 & 0.62 \\
\hline [Gly $\left.{ }^{22}\right](19-31) \operatorname{PrRP}$ & $W$ & $Y$ & A & G & $\mathrm{R}$ & G & I & $\mathrm{R}$ & $\mathrm{P}$ & $\mathrm{V}$ & G & $\mathrm{R}$ & $\mathrm{F}$ & $-\mathrm{NH}_{2}$ & 0.0078 & 0.01 \\
\hline$\left[\mathrm{Thr}^{21}\right](19-31) \operatorname{PrRP}$ & $W$ & $\mathrm{Y}$ & $\mathbf{T}$ & S & $\mathrm{R}$ & G & I & $\mathrm{R}$ & $\mathrm{P}$ & $\mathrm{V}$ & G & $\mathrm{R}$ & $\mathrm{F}$ & $-\mathrm{NH}_{2}$ & 0.0068 & 0.02 \\
\hline
\end{tabular}

${ }^{a}$ Data are taken from Boyle et al., 2005.

$\mathrm{K}_{\mathrm{i}}$ values are from competition binding studies, with $\mathrm{Eu}-(\mathrm{Lys}) \operatorname{PrRP} 31$ as a tracer, while $\mathrm{EC}_{50}$ values were determined in a calcium mobilization assay using HEK293-ASR-1 cells. 
Table 7. Binding affinity $\left(K_{i}\right)$ and functional potency $\left(\mathrm{EC}_{50}\right)$ at Kiss1R of kisspeptin-10 and kisspeptin-13 derived peptides. ${ }^{\text {a }}$

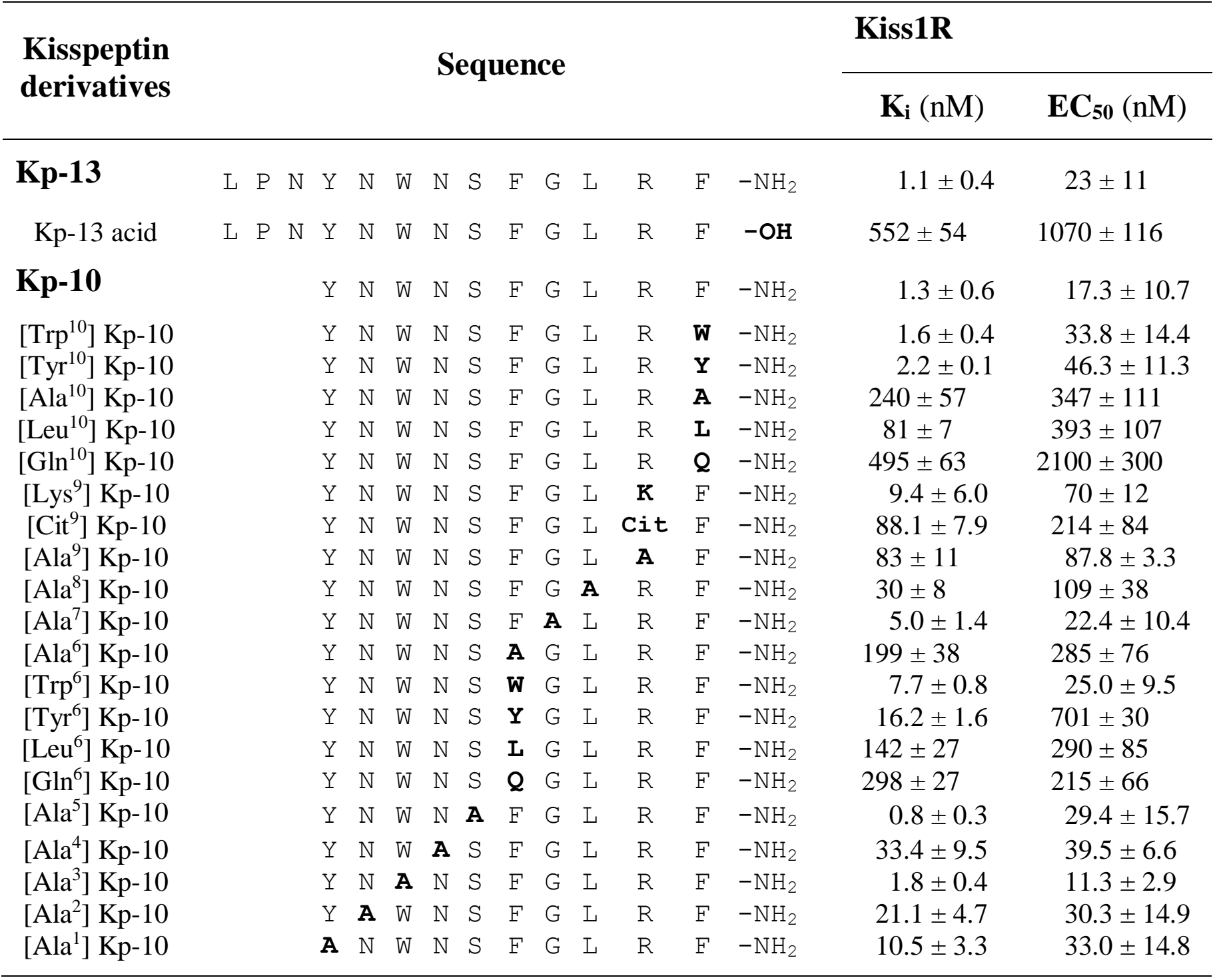

${ }^{a}$ Data are taken from Orsini et al., 2007.

For a sake of clarity, kisspeptin-10 and kisspeptin-13 derivatives are abbreviated as Kp-10 and Kp-13, respectively. $\mathrm{K}_{\mathrm{i}}$ values are from competition experiments performed on Kiss1R- (GPR54) containing membranes, using $\left[{ }^{125} \mathrm{I}\right]$-metastin as the tracer. $\mathrm{EC}_{50}$ values are from a functional calcium mobilization assay performed on Kiss1R-expressing HEK293 cells. 
Figure 1.
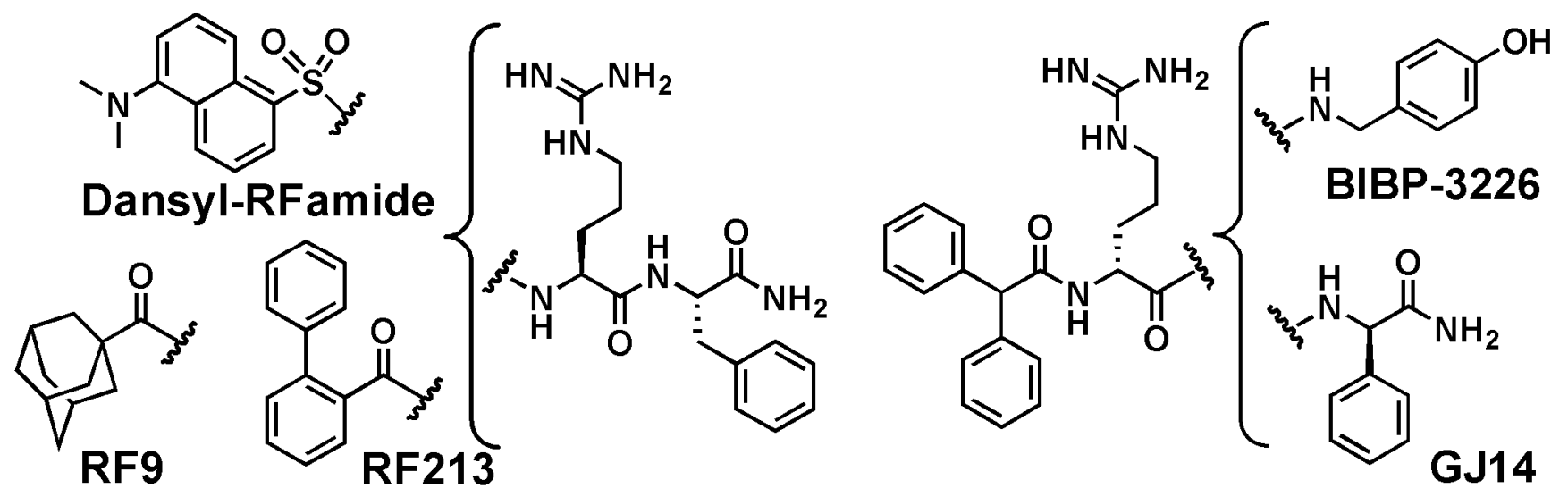<smiles>[R][R5]([R])([R])C(=O)N(C)CCCNC(=N)N</smiles>

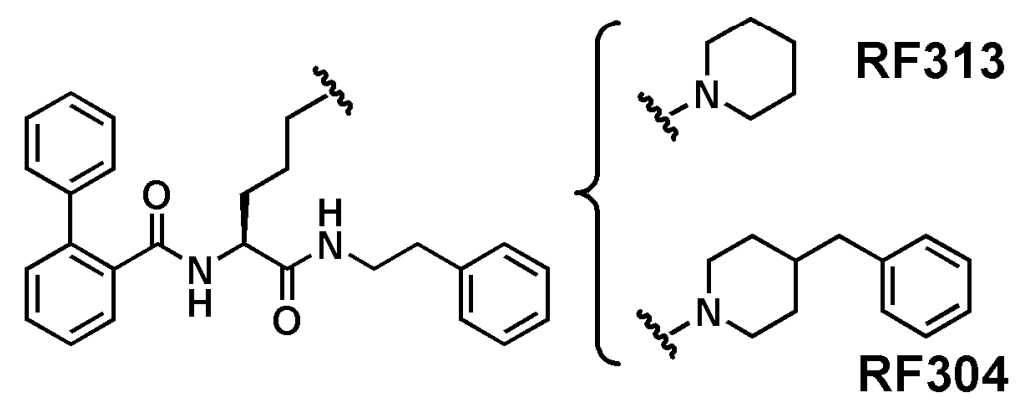

125 
Figure 2.

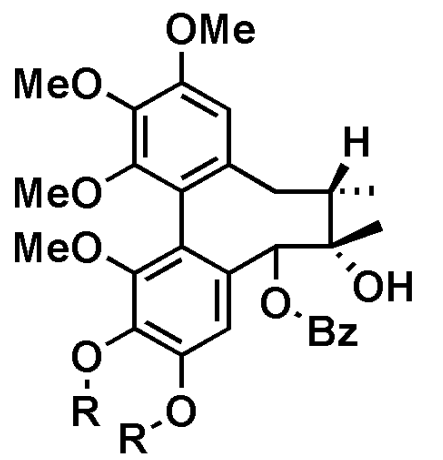<smiles>[R2]c1nc(NC(=N)N)sc1[R]</smiles>

Actelion

Pharmaceuticals<smiles>[R][R]1ccc2nc(NC(=N)N)[X]c(C)c2c1</smiles>

Synaptic Pharmaceuticals

Benzoyl Gomisin Q : R = Me Gomisin G : $\mathbf{R}=-\mathrm{CH}_{2}$ -<smiles>N=C(N)NN=CC1=CC=C2[R]#CC=C21</smiles>

Acadia Pharmaceuticals<smiles>N=C(N)NCCNC(=O)C1(Nc2ccccc2)CCN(Cc2ccccc2)CC1</smiles>

Cpd 46<smiles>N=C(N)NCC(=O)NCC1(C[R16]([NH3+])=O)CCN(Cc2ccccc2)CC1</smiles>

Cpd 53a 
Figure 3.<smiles>COn1c(N[C@H](C)c2ccc(C(F)(F)F)cc2)nc2c(c1=O)CN(C(=O)c1ccc(C#N)cc1)CC2</smiles>

Cpd 2386

$\mathrm{Ki}=2 \mathrm{nM}$ 
Figure 4.

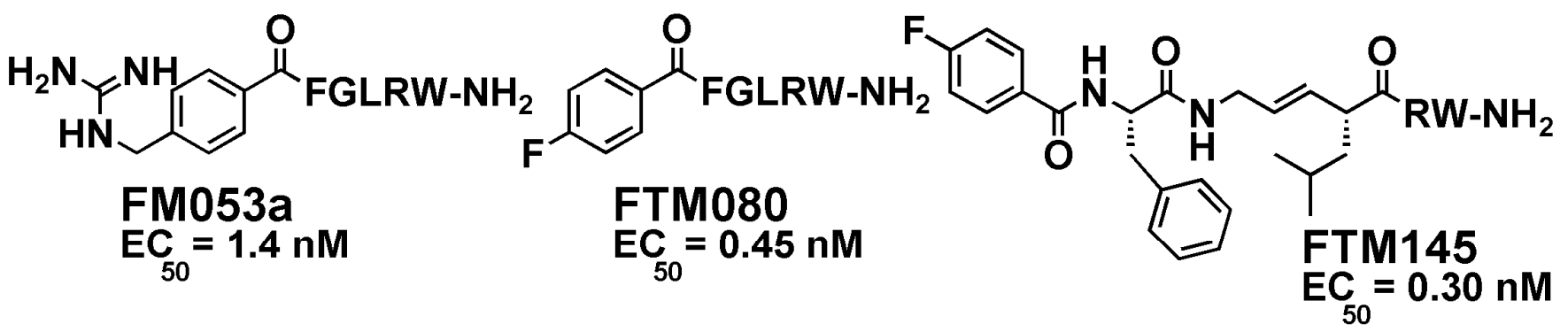

TAK-448<smiles>CCCCCC(=O)C(Cc1ccc(O)cc1)NC(C)=O</smiles>

$\mathrm{EC}_{50}=0.30 \mathrm{nM}$

$\mathrm{EC}_{50}=0.33 \mathrm{nM}$<smiles>CCCCNC(=N)NCCC(NC(=O)[C@H](CC(C)C)NC(=O)NNC(=O)[C@H](Cc1ccccc1)NC(=O)[C@H](NC(=O)[C@H](CC(N)=O)NC(=O)[C@H](Cc1c[nH]c2ccccc12)NC(=O)[C@H](Cc1c[nH]c2ccccc12)NC(C)=O)C(C)O)C(N)=O</smiles>

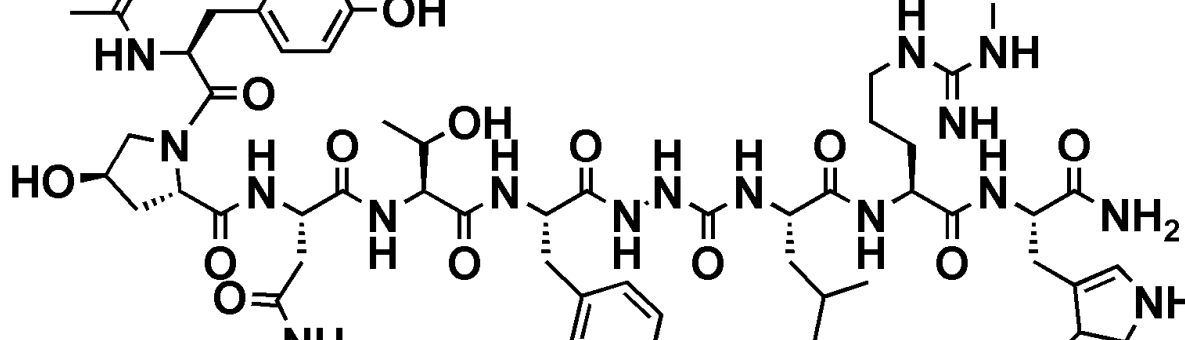
$\mathrm{NH}_{2}$<smiles>CC(C)C=O</smiles>

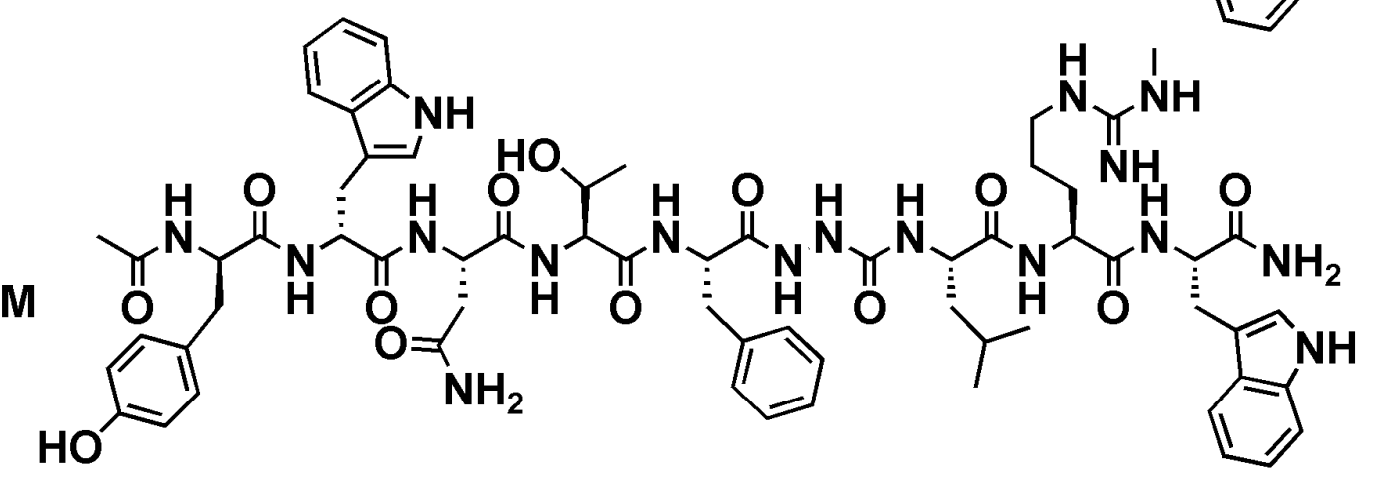<smiles>Cc1ccccc1</smiles>

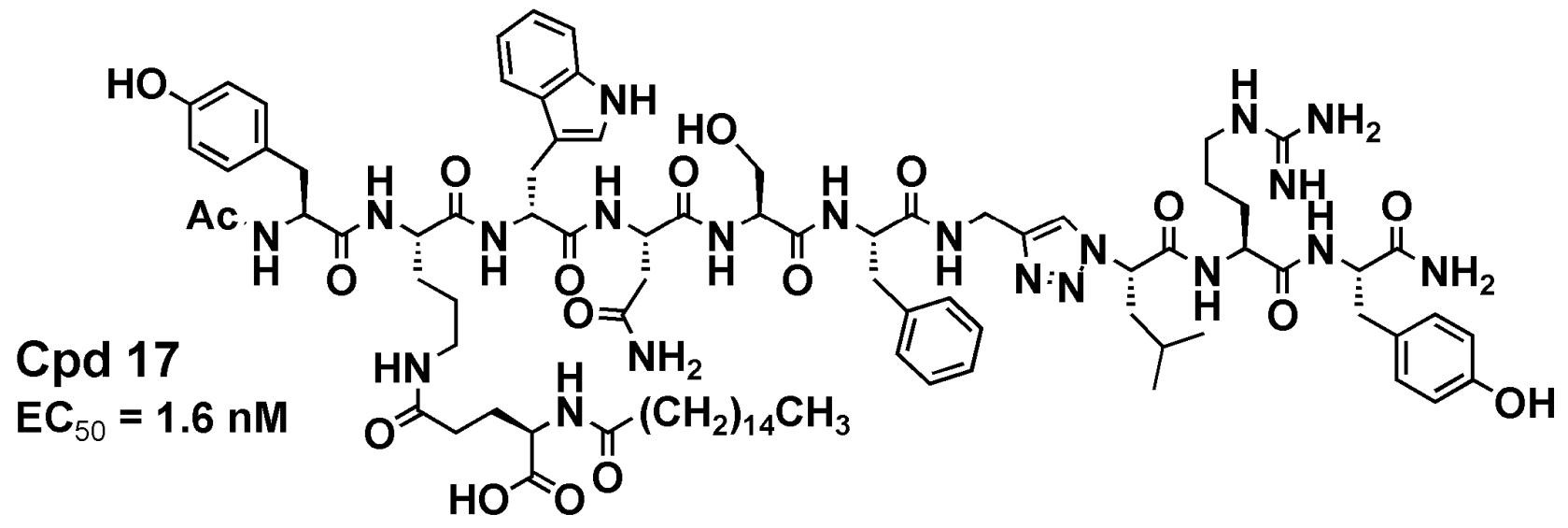


Figure 5.

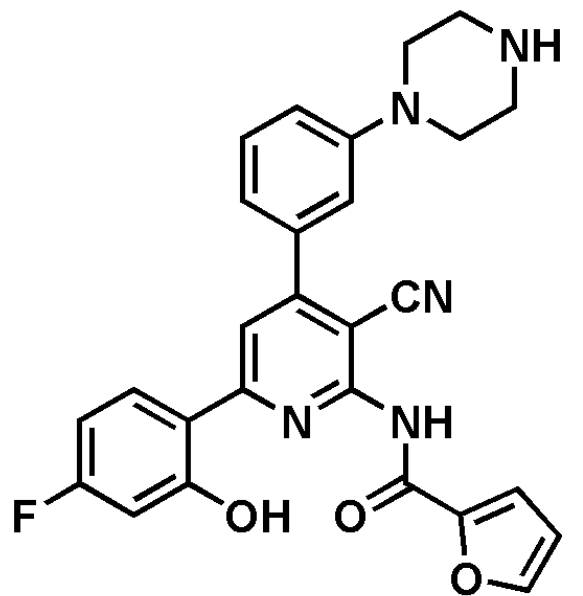

$$
I C_{50}=3.6 \mathrm{nM}
$$<smiles>N#Cc1c(-c2cccc(NC(=O)CCN)c2)cc(-c2ccc(F)cc2O)nc1NC(=O)c1ccco1</smiles>

$$
\mathrm{IC}_{50}=3.7 \mathrm{nM}
$$


Figure 6.

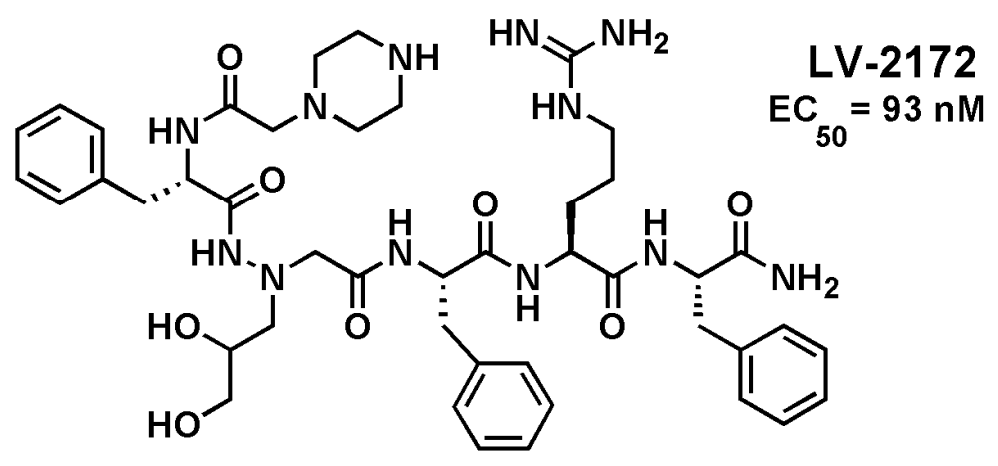

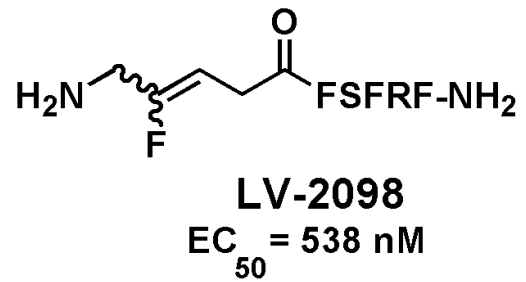<smiles>CN(C)Cc1ccc2c(c1)CCN(C(=O)c1cc3cc(Cl)ccc3n1C)C2</smiles>

Indole derivative (Banyu Pharmaceuticals) $\mathrm{IC}_{50}=6.1 \mathrm{nM}$<smiles>CC1CN(C(=O)c2cc3cc(Br)ncc3n2C)Cc2ccc(CN(C)C)cc21</smiles>

Pyrrolo[2,3-c]pyridine 25e (AstraZeneca) $\mathrm{IC}_{50}=40 \mathrm{nM}$<smiles>COc1ccc(Cl)cc1NS(=O)(=O)c1ccc(OC)c2c1CC[C@H](N(C)C)C2</smiles>

Sulfonamide 2 (AstraZeneca) $\mathrm{IC}_{50}=80 \mathrm{nM}$ 
Figure 7.

RFRPs

NPFF1R

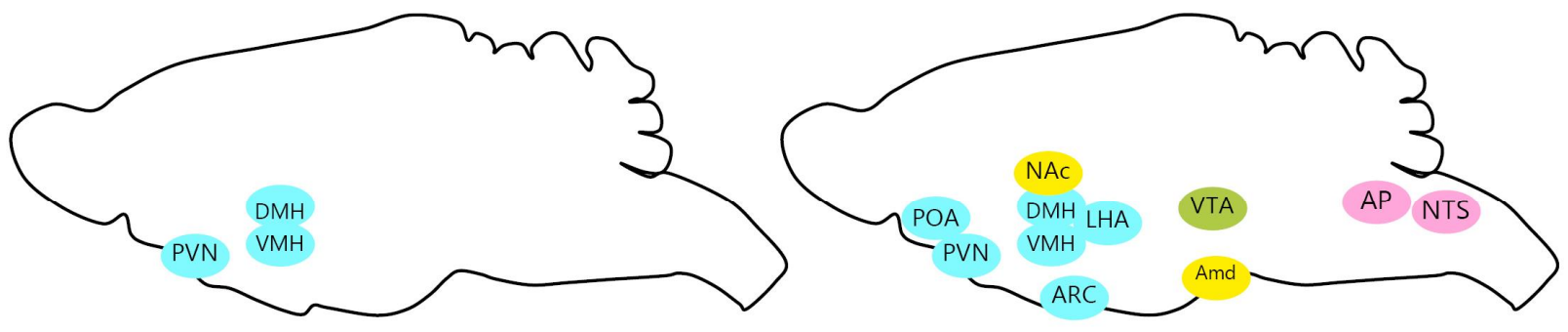

NPFF/NPAF

NPFF2R

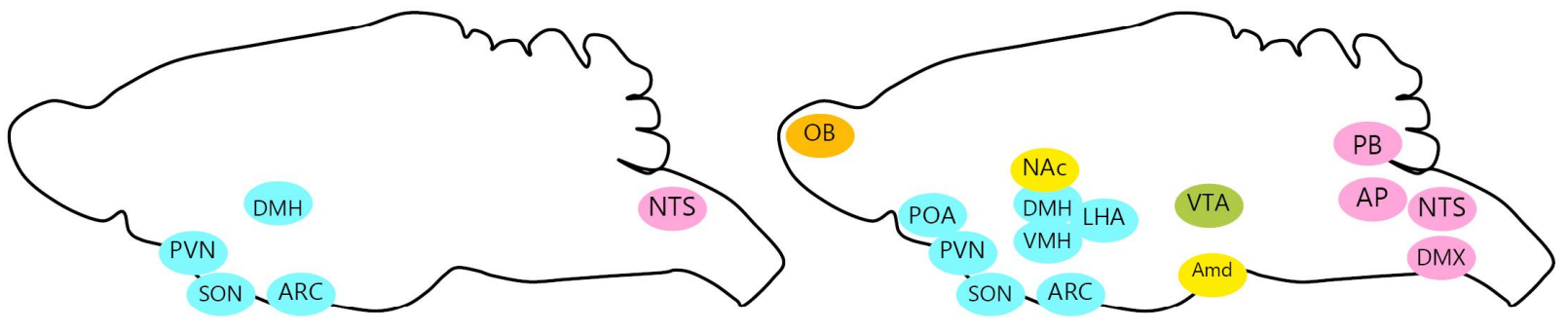

PrRPs

PrRP-R

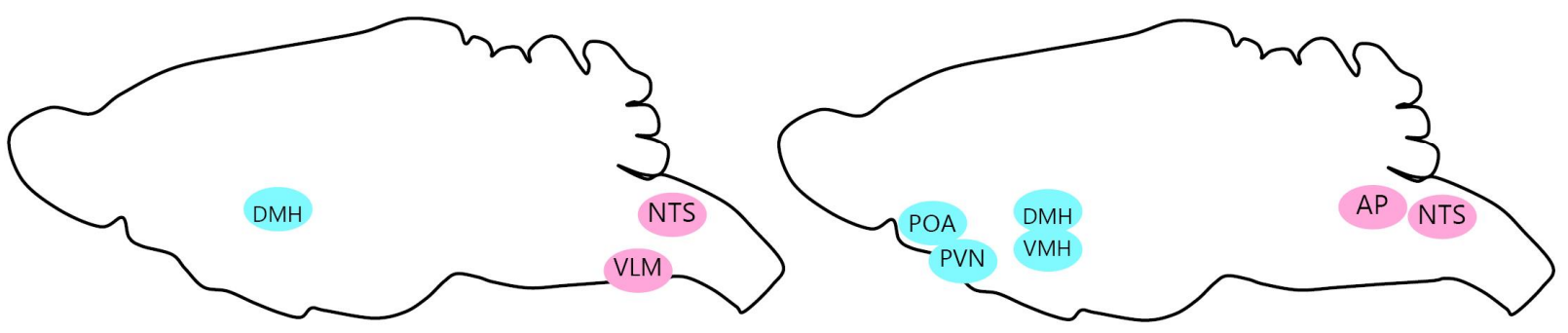

Kisspeptins

Kiss1R

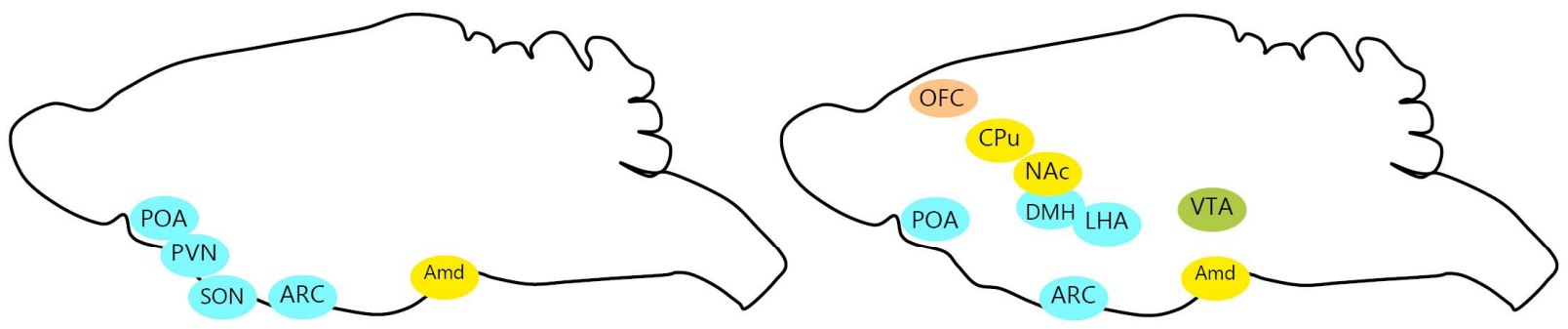

QRFPs

QRFP-R

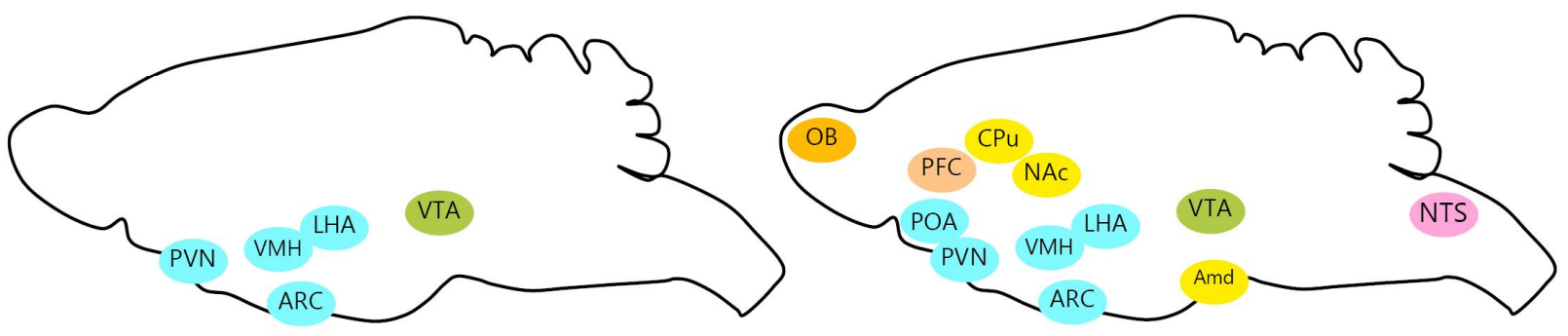


Figure 8.

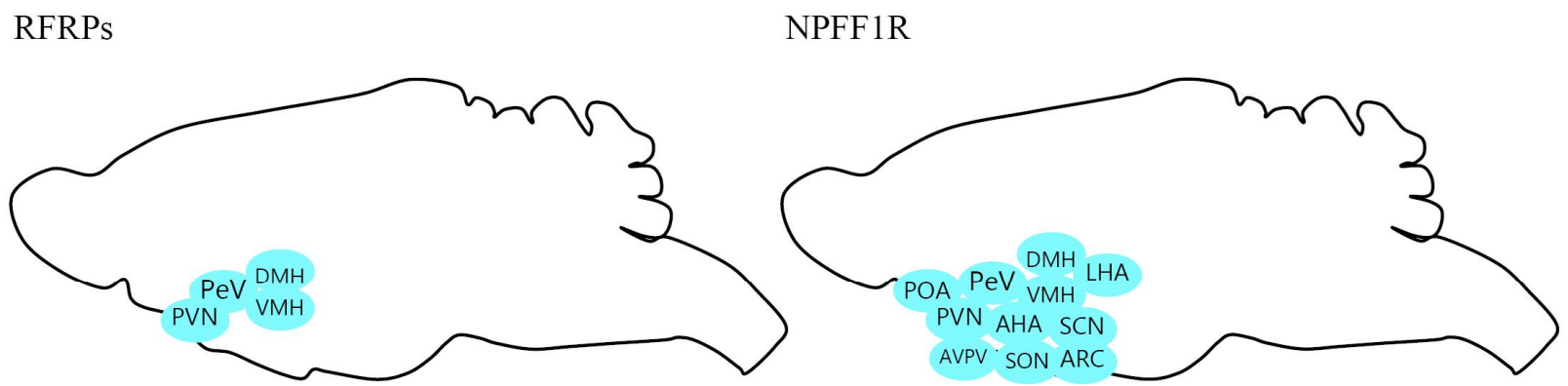

PrRPs

PrRP-R

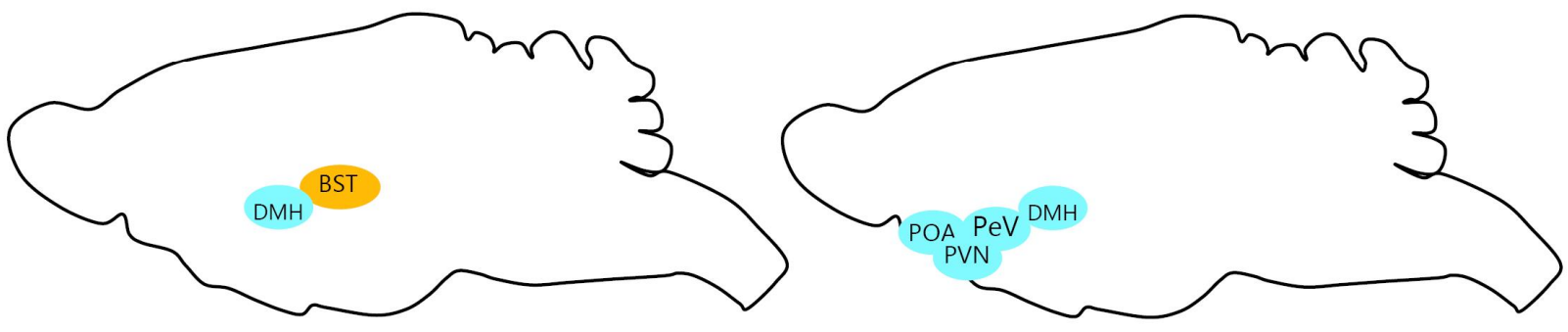

Kisspeptins

Kiss1R

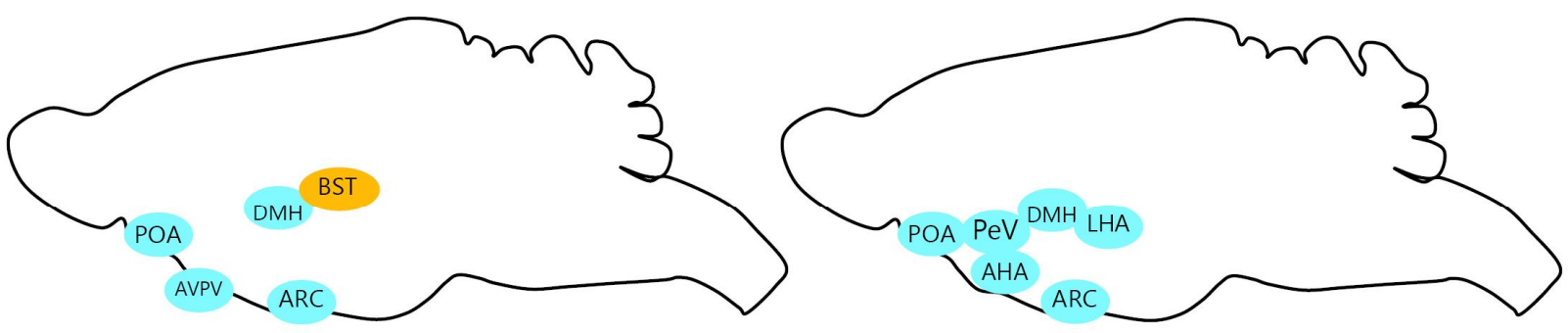

QRFPS QRFP-R

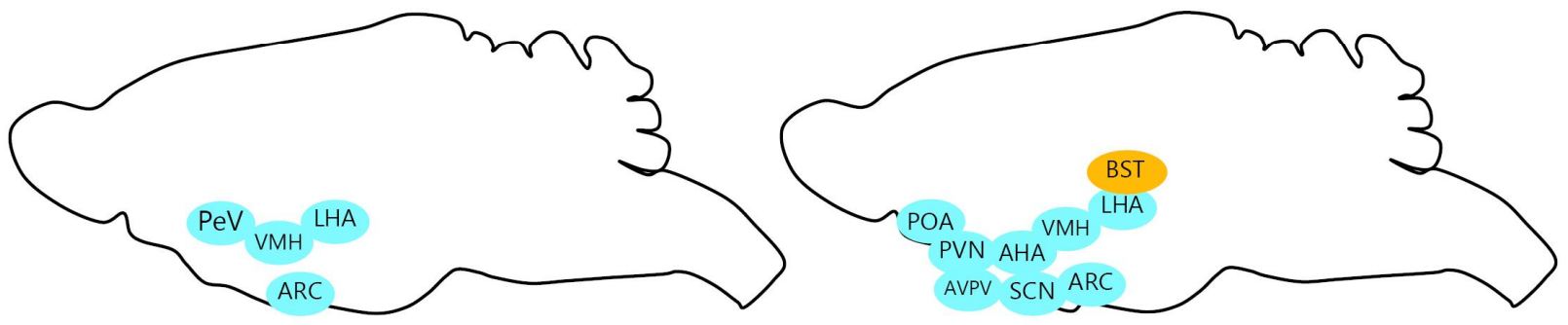




\section{Figure 9.}

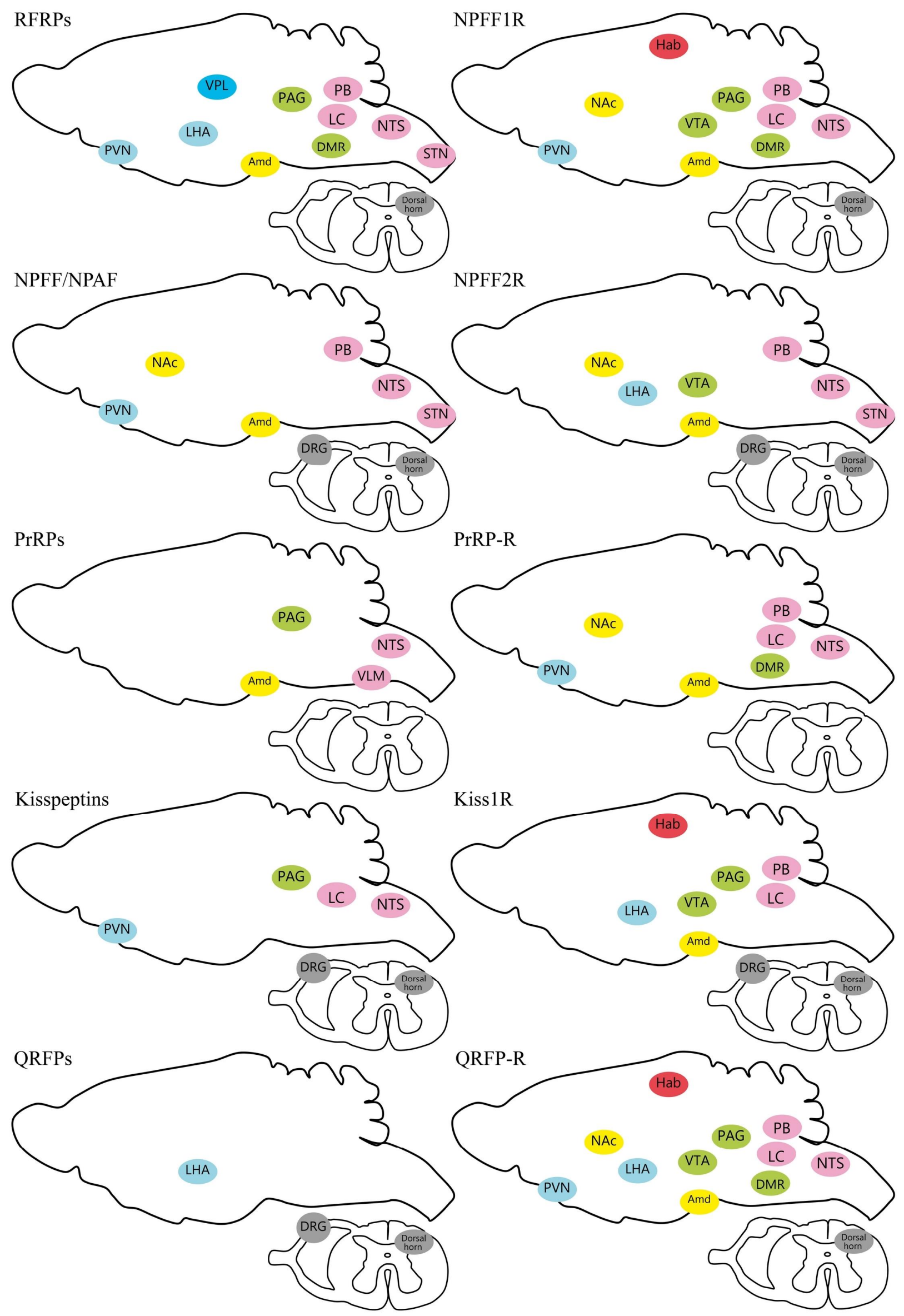

